



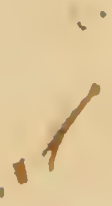

(6)

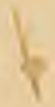




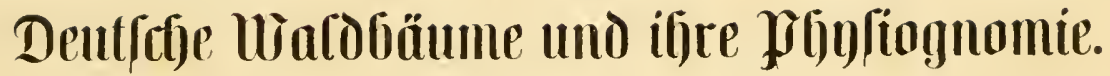




$\begin{array}{lll}4 & \end{array}$

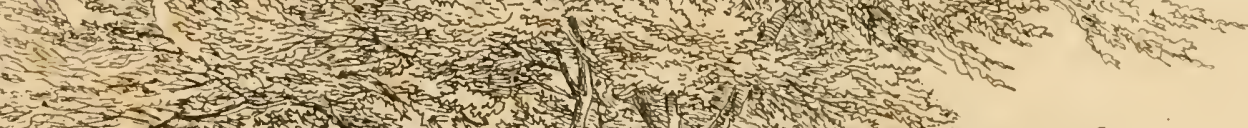

3 ,

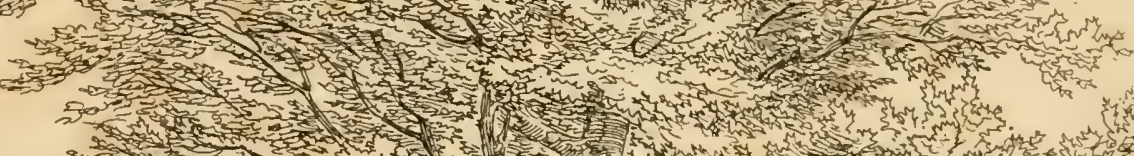

(n)

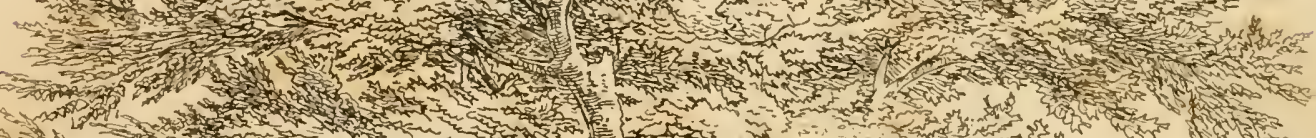

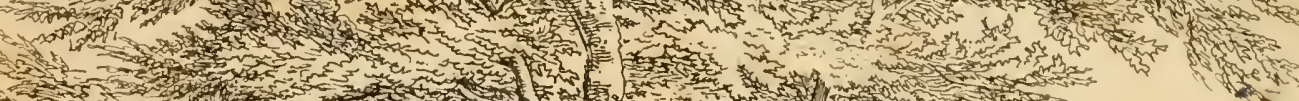

and

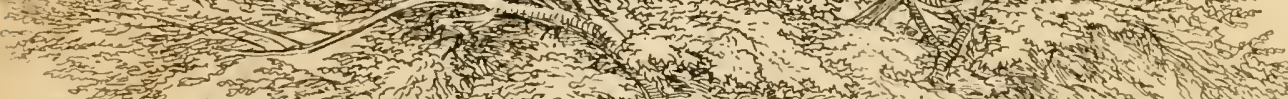

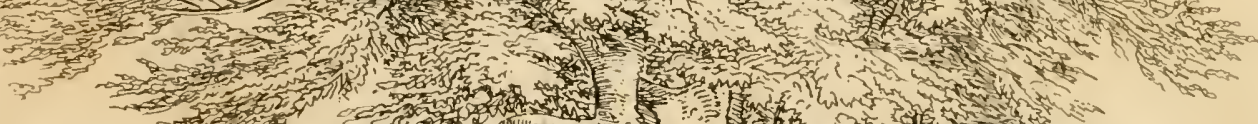

2.

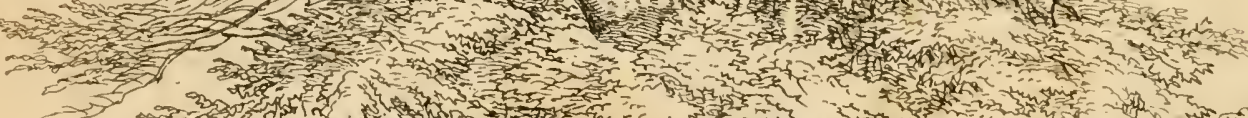

5.

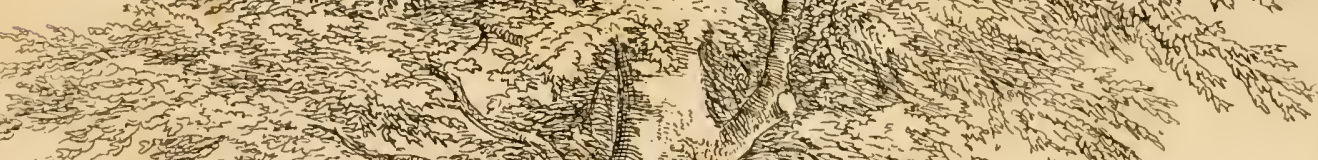

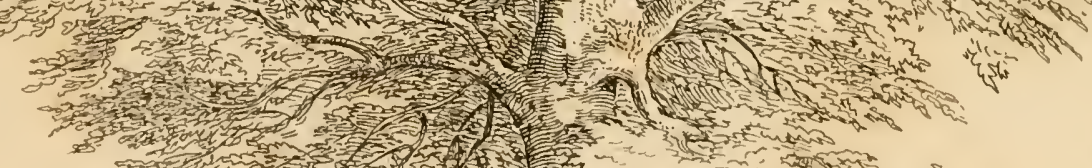

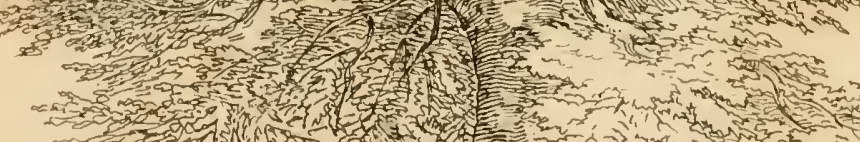

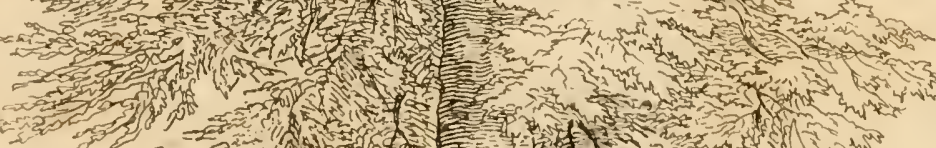

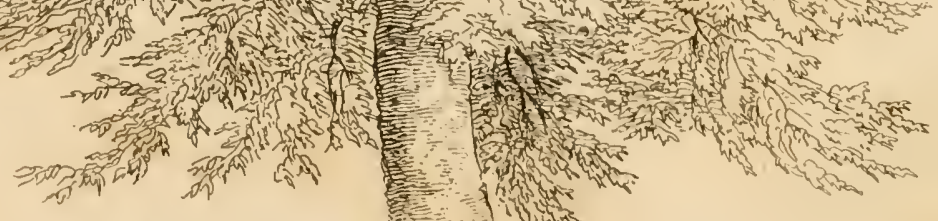




\section{Dentride}

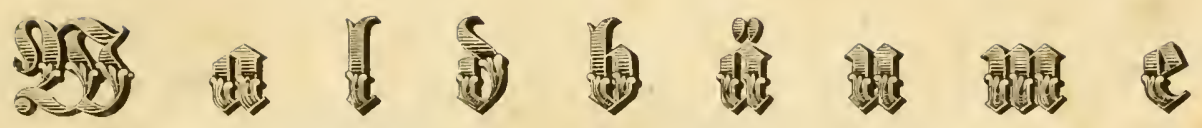

\section{und ifre fignfiognomie.}

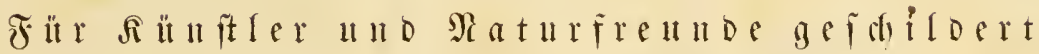
พอII

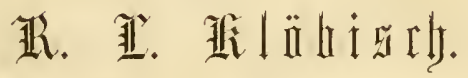

Mit

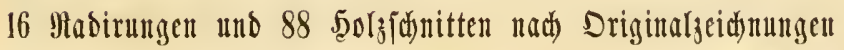

vou

w. f). 迎berhard.

IILixjit

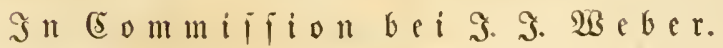

18.57. 


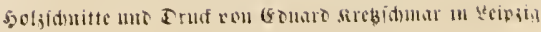




\section{Vorwart des forrangebers.}

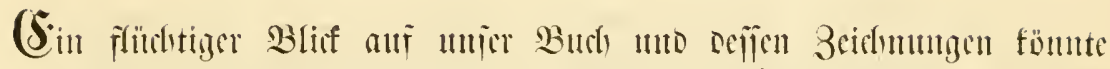

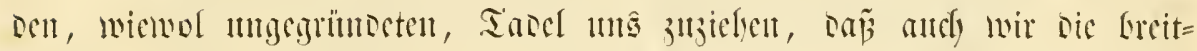

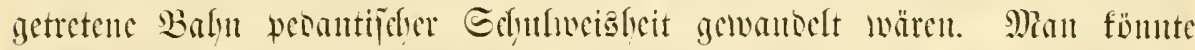

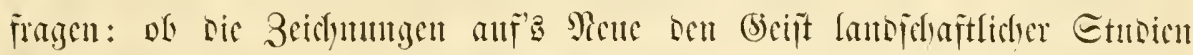

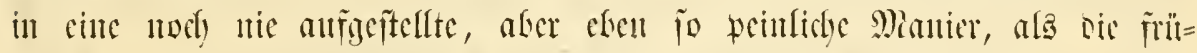

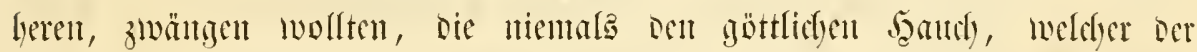

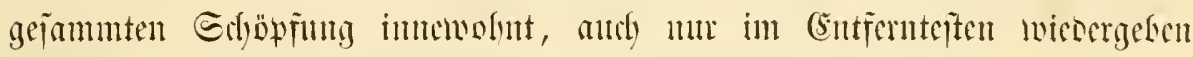
fönnte.

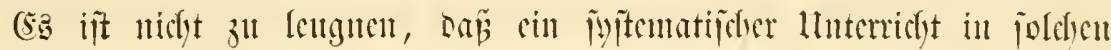

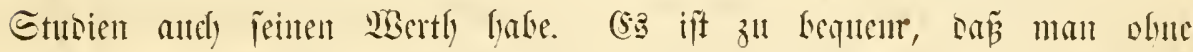

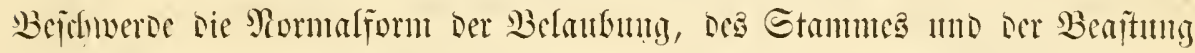

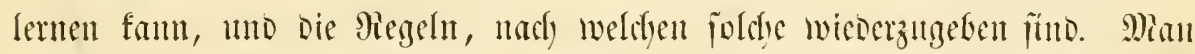

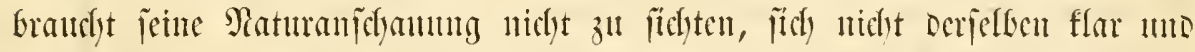

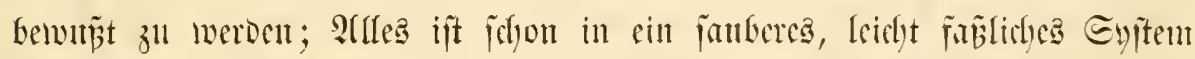

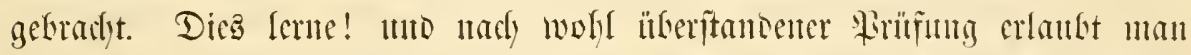

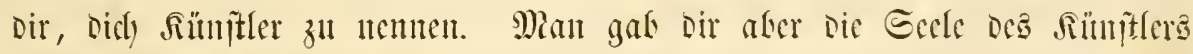
nictyt zutr Mitrgīt.

\footnotetext{
"98em Gntt will cine Gunit ermaīen,

Dell idfirft or in vic weite 2 selt,

Dem will or feine whunter weifen

In Felo unt sूale uno Etrum unt Felt."
}

Sn Diejen :

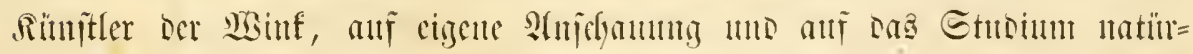
licter Driginale fisty mur żu itüben. 


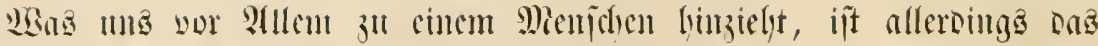

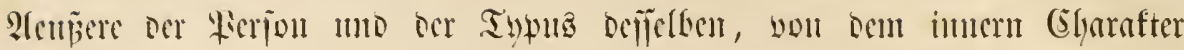

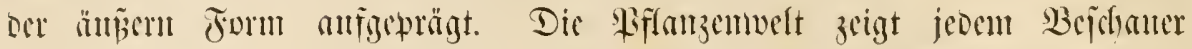

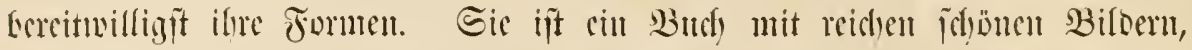

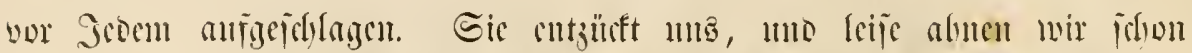

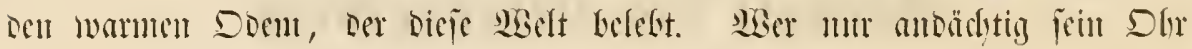

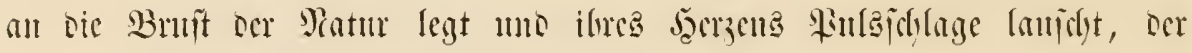

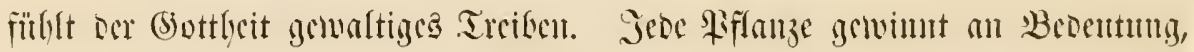

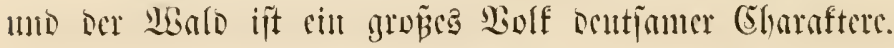

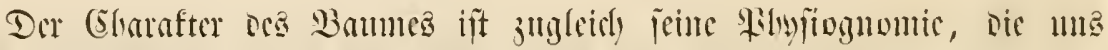

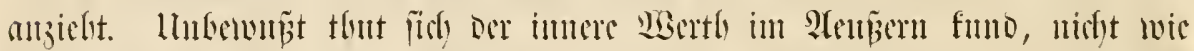

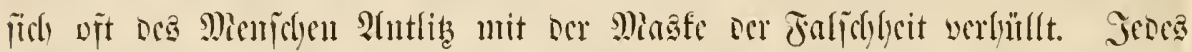

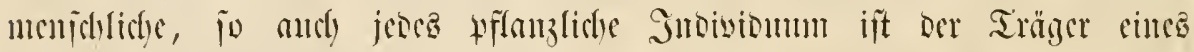

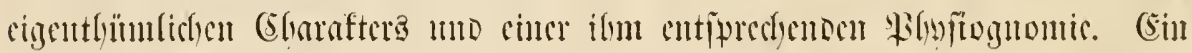

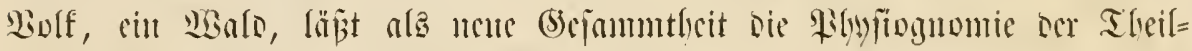

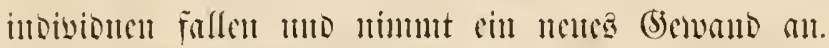

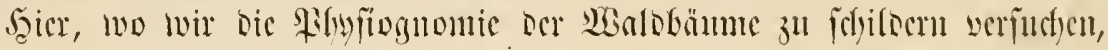

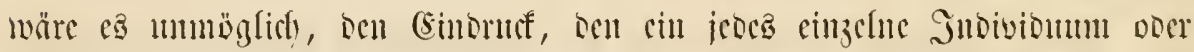

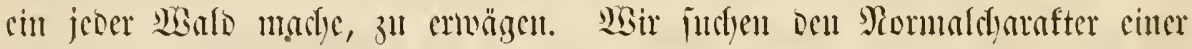

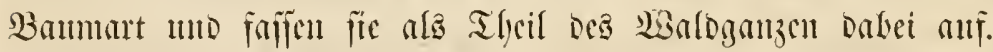

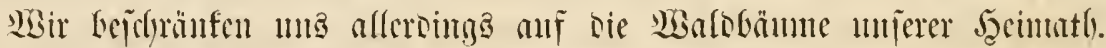

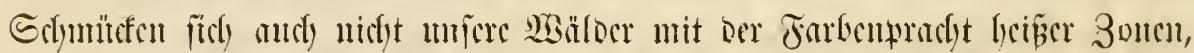

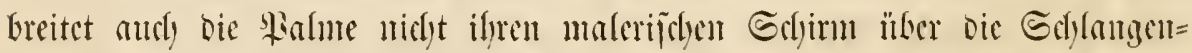

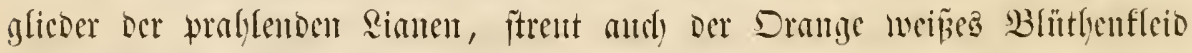

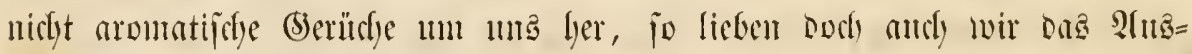

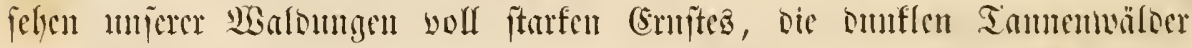

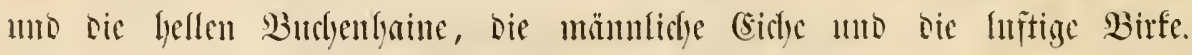
Slunen find dieje Zcilen geneilyt.

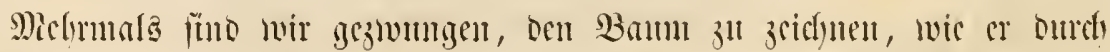

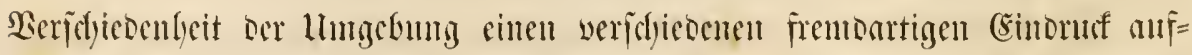

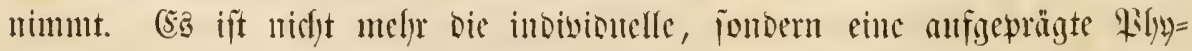

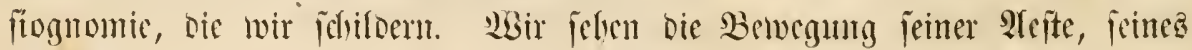


Dí de

$$
\text { utis ifre }
$$

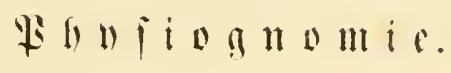





\section{(5) $i \mathfrak{l l} \mathfrak{l} i t \| \mathbb{t} \mathfrak{g}$.}

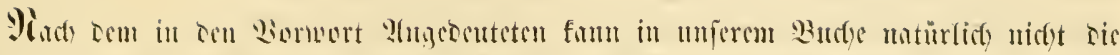

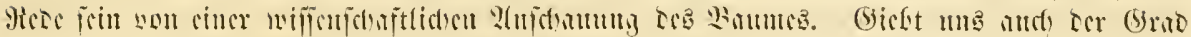

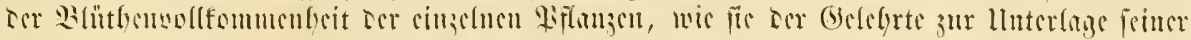

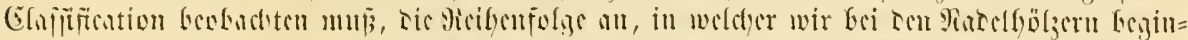

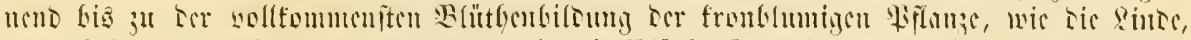

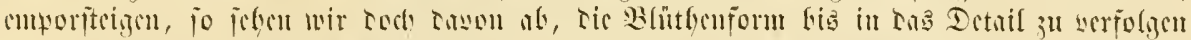

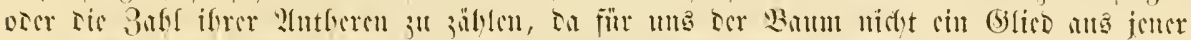

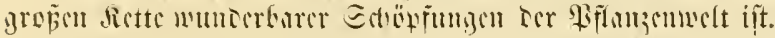

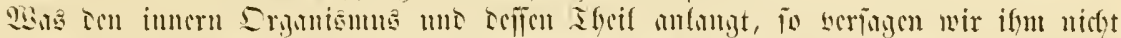

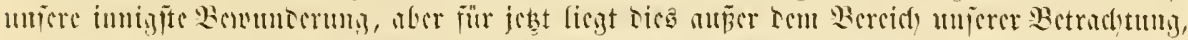

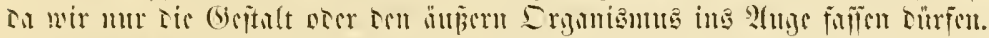

Ins (Emtrum Secjes

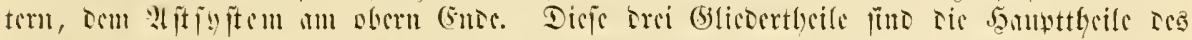

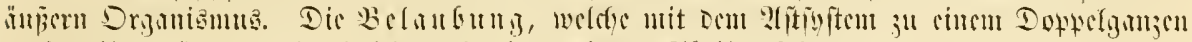

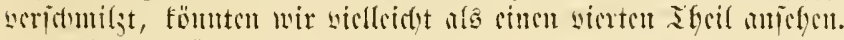

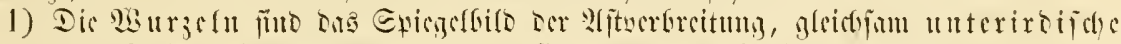

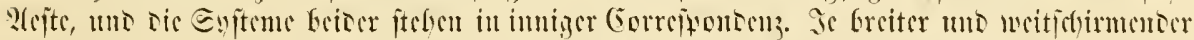

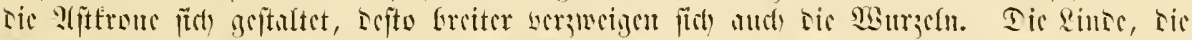

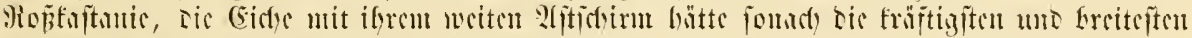

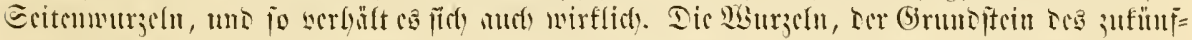

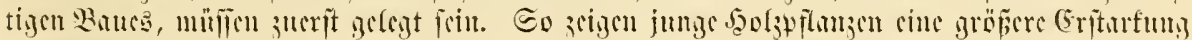

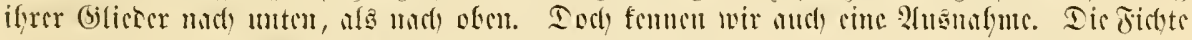

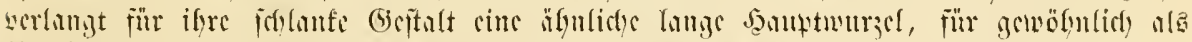

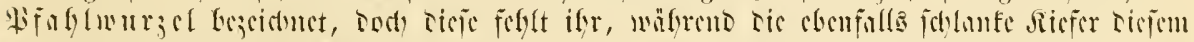

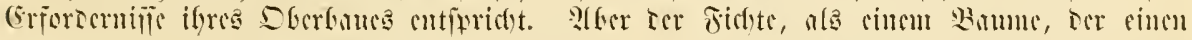

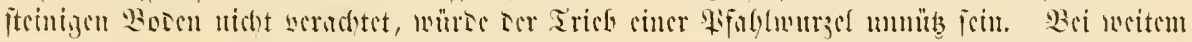

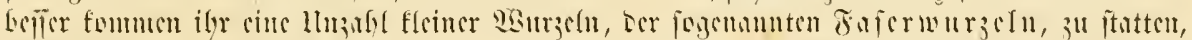

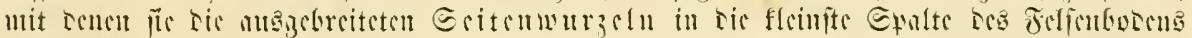

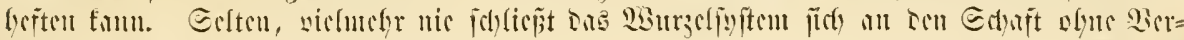

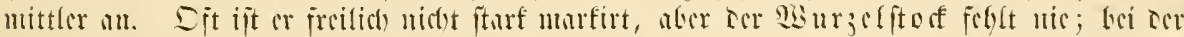

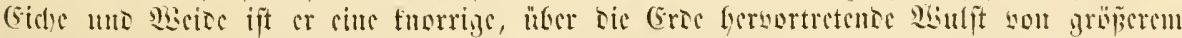

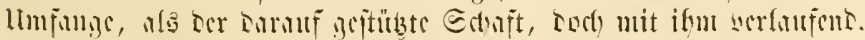

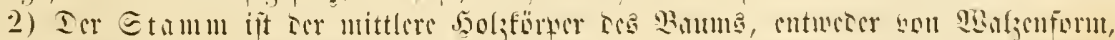

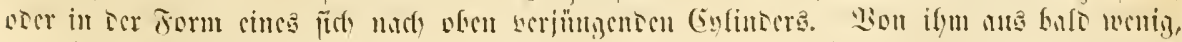

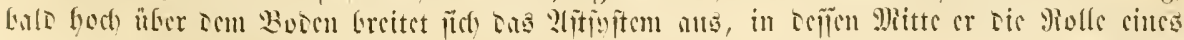

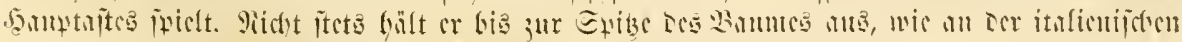

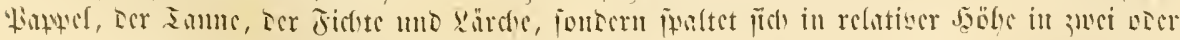

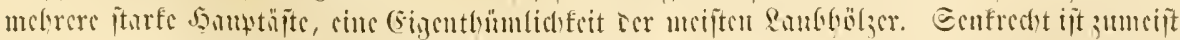

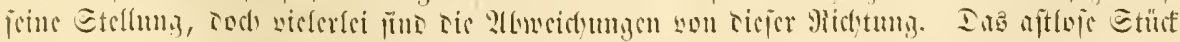

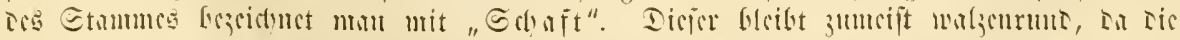

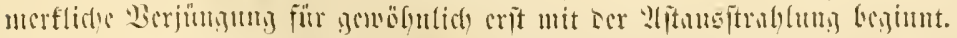

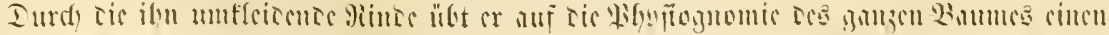

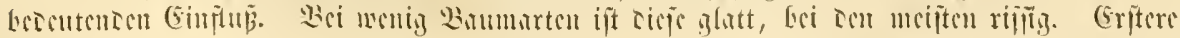




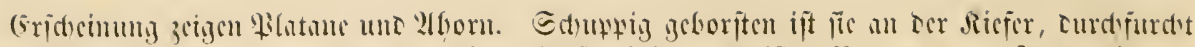

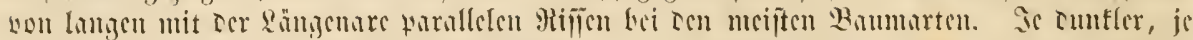

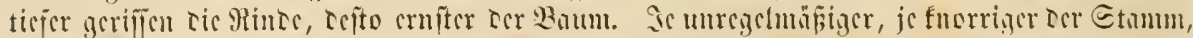

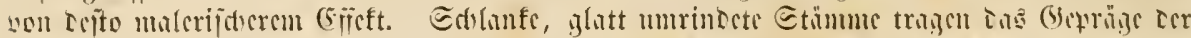

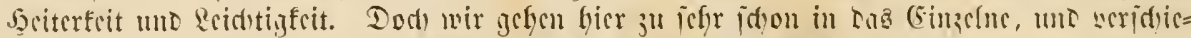

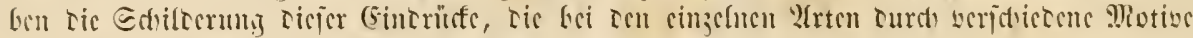

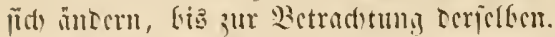

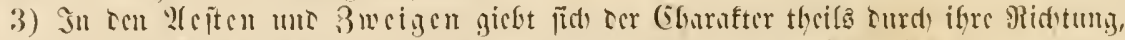

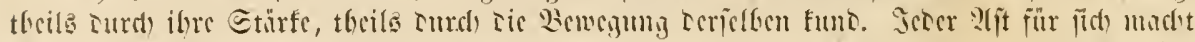

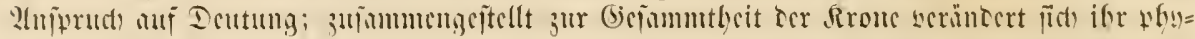

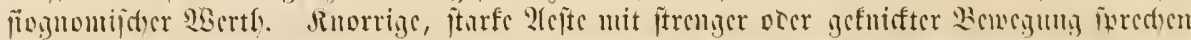

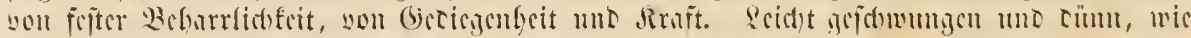

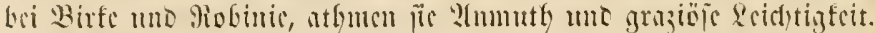

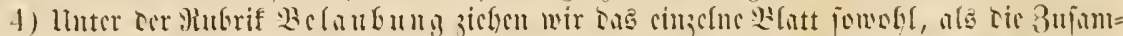

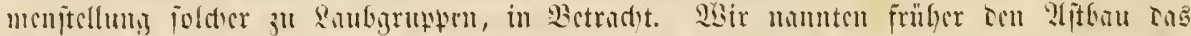

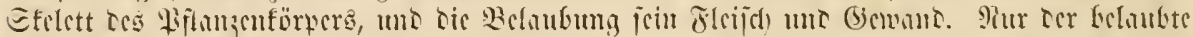

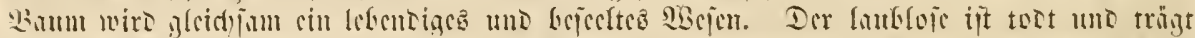

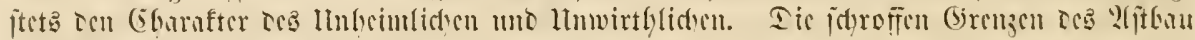

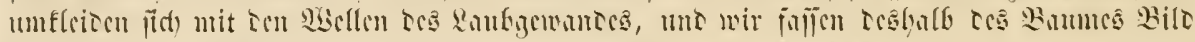

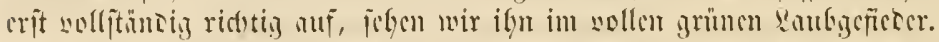

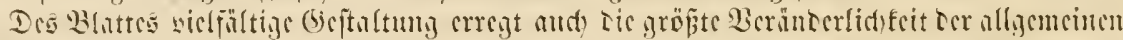

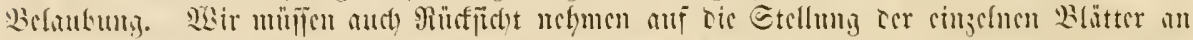

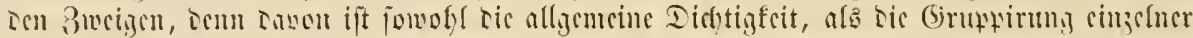

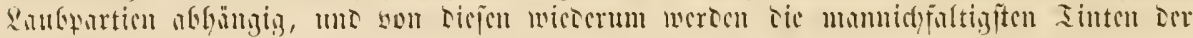
Zanturingumb betimit.

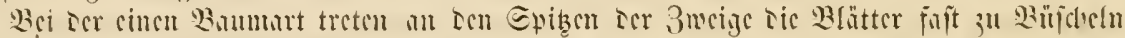

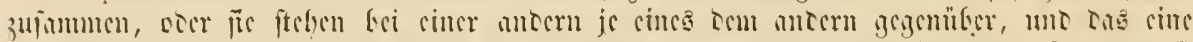

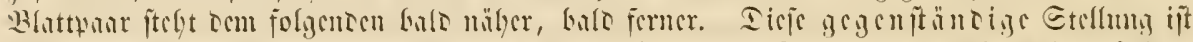

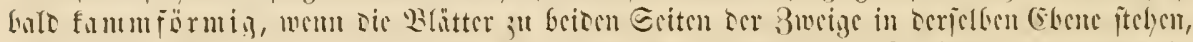

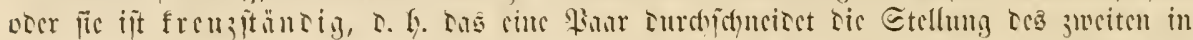

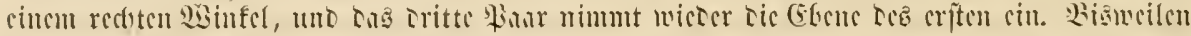

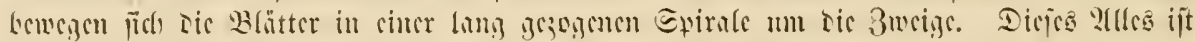

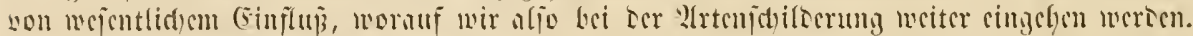

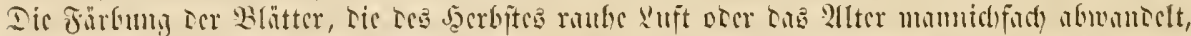

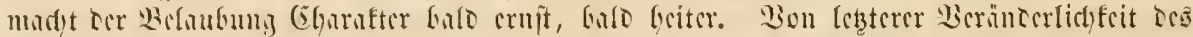
grünen (S)

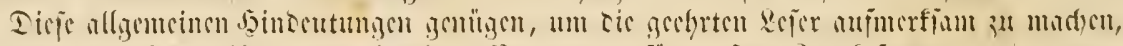
แล ton rïrion.

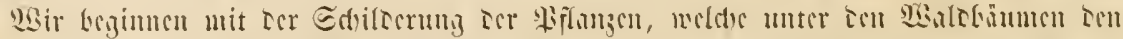

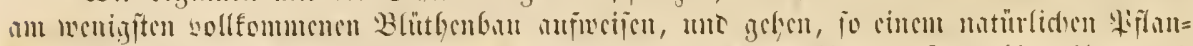

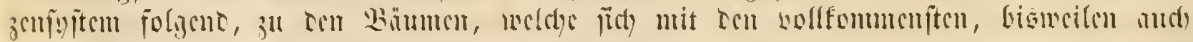

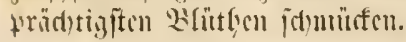




\title{
Die Jùdeliälor.
}

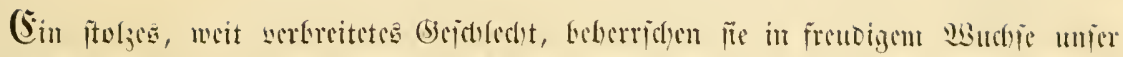

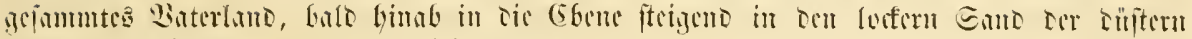

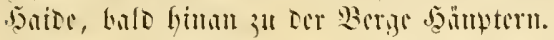

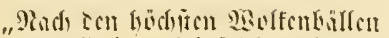

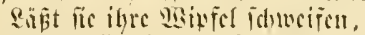

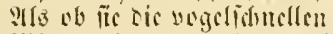 \\ Mlit cen Prmen wellte greifon." \\ (Friligrath.)
}

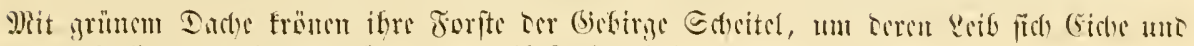

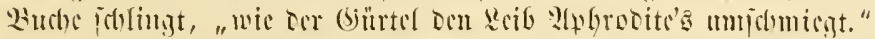

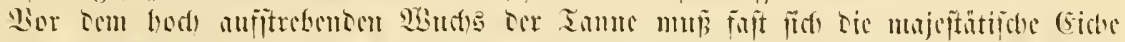

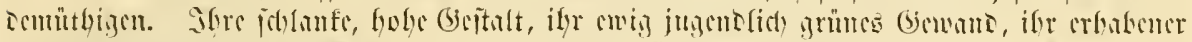

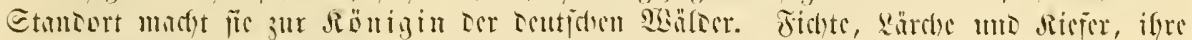

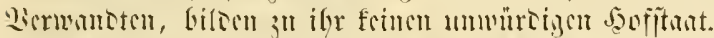

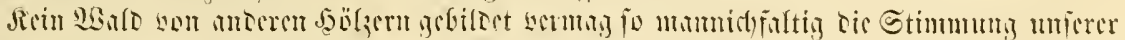

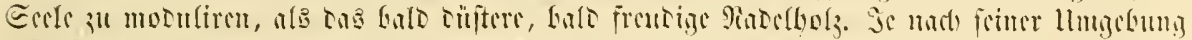

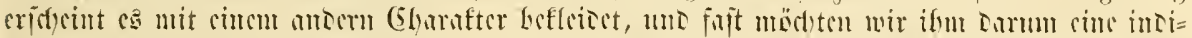

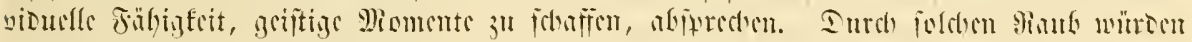

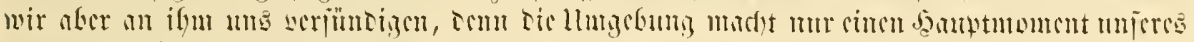

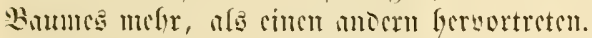

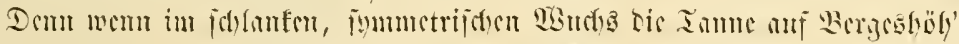

$$
\begin{aligned}
& \text { "Etill find itrecft, } \\
& \text { soed ind refft } \\
& \text { itt zic "uft," }
\end{aligned}
$$

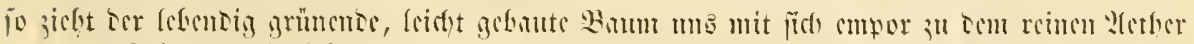

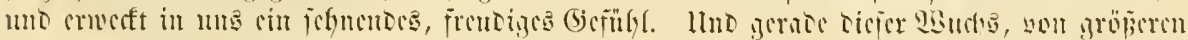

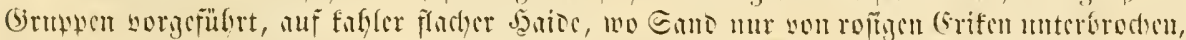

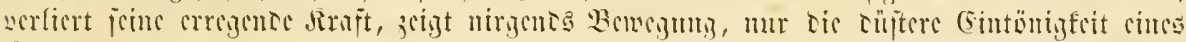
Gimlimuloca.

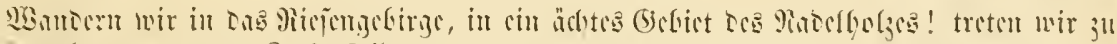

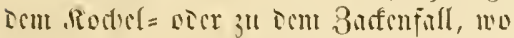

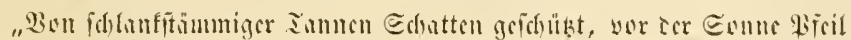

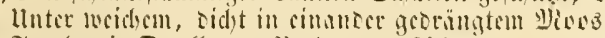

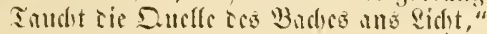

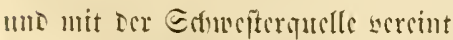

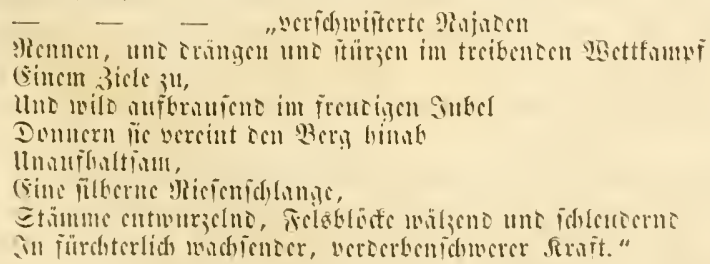

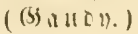




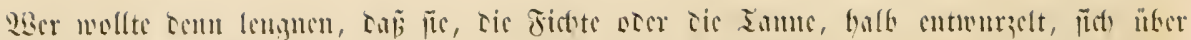

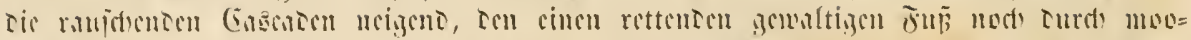

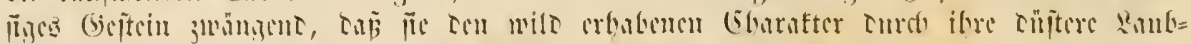

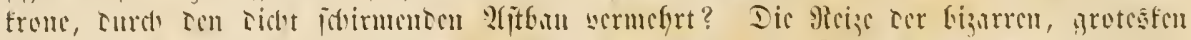

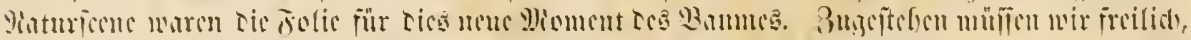

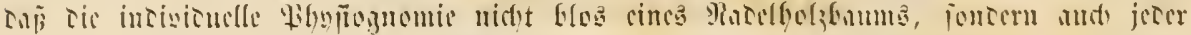

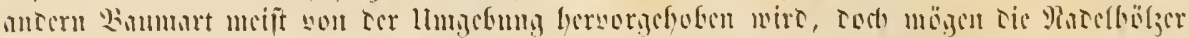

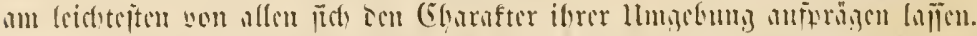

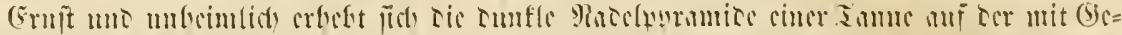

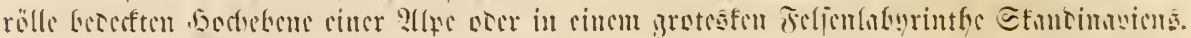

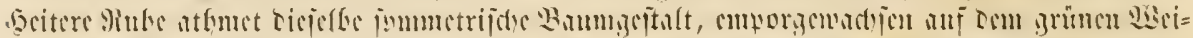

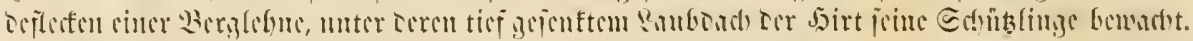

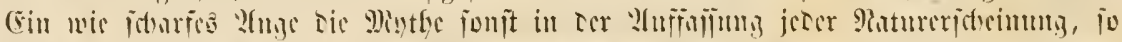

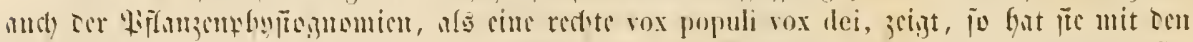

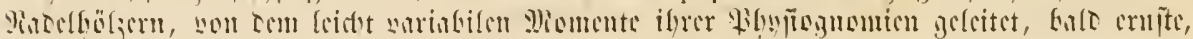

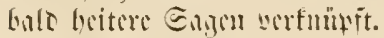

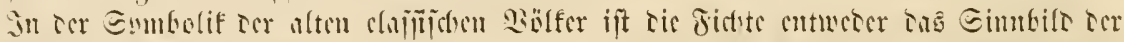
Jrente, ocer tro Estumerzas.

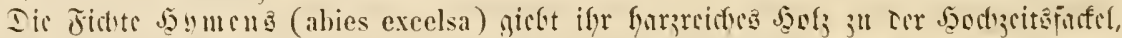

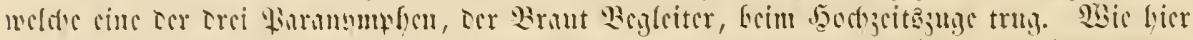

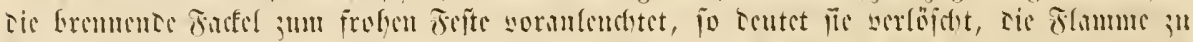

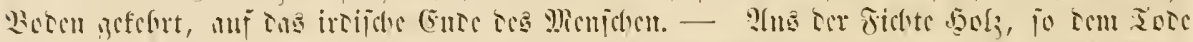

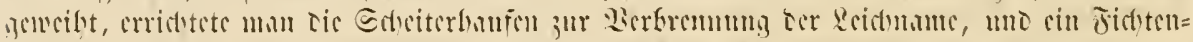

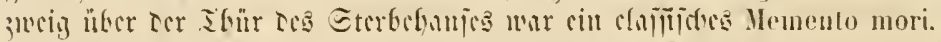

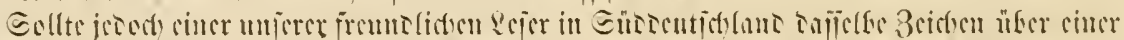

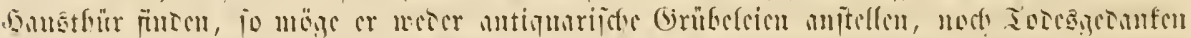

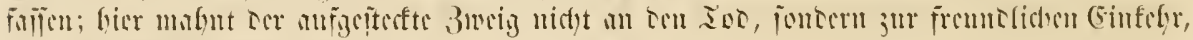
3um edet Eentichen (Soritentranf.

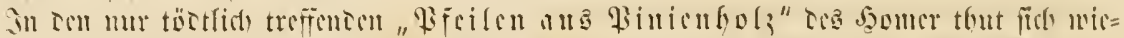

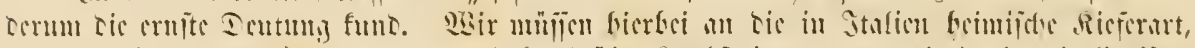

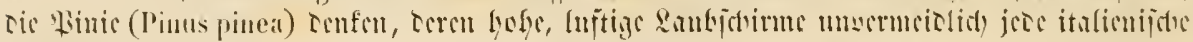

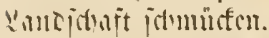

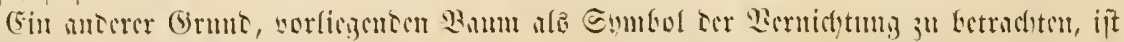

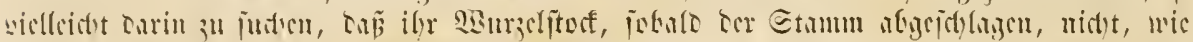

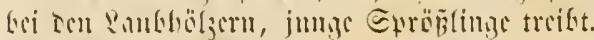

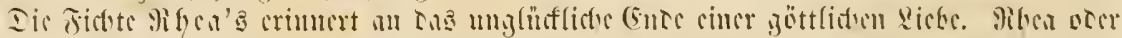

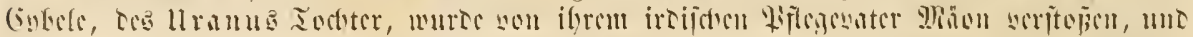

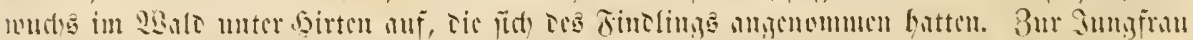

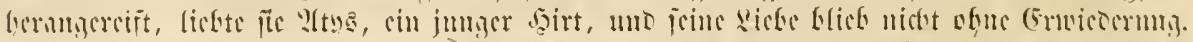

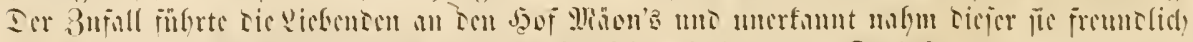

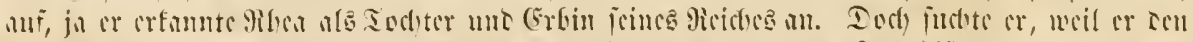

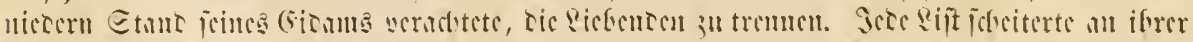

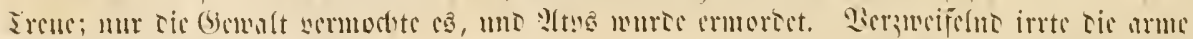

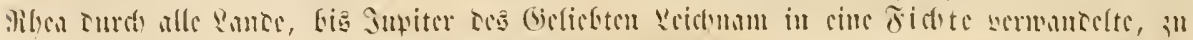

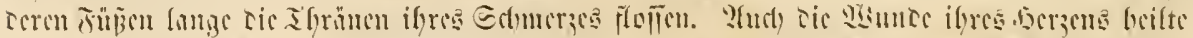

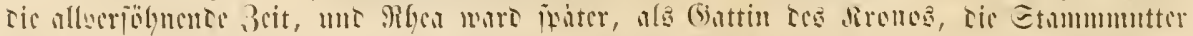

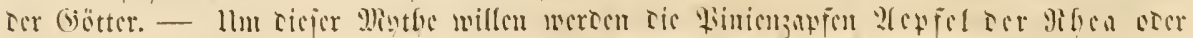
Ter Gufole gumomt.

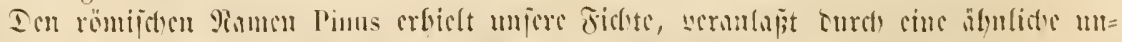

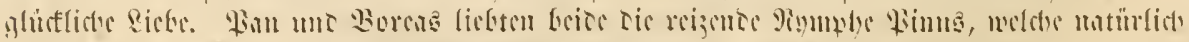

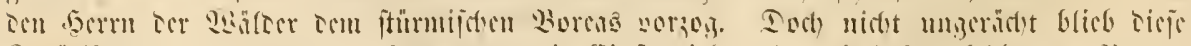

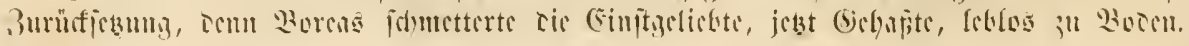




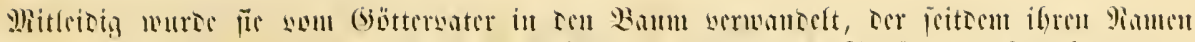

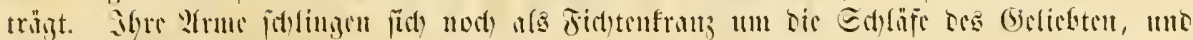

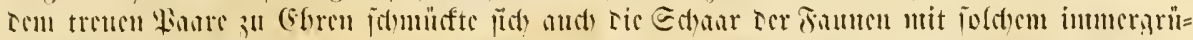

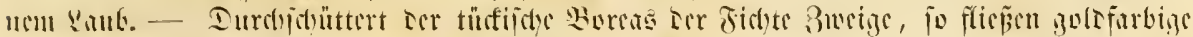
Shrïnch wou ibren Equizen.

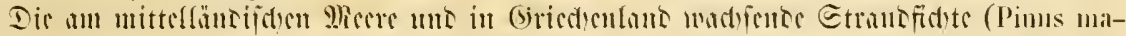

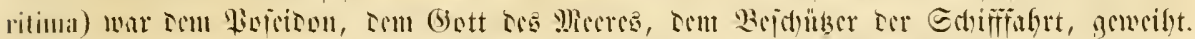

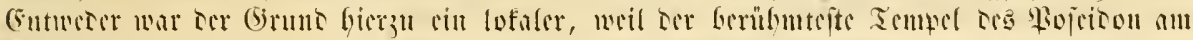

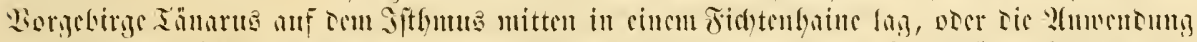

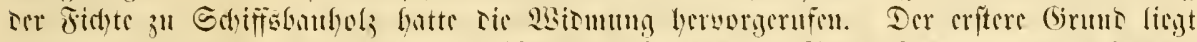

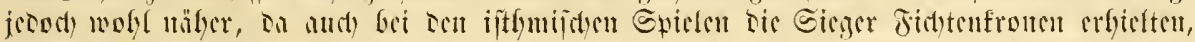

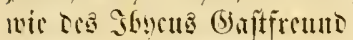

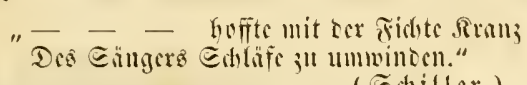

(E)iller.)

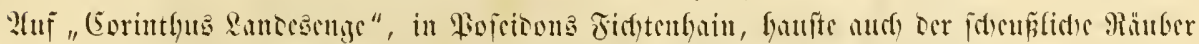

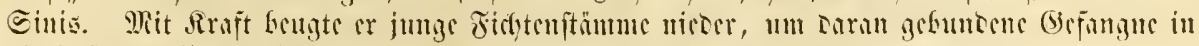

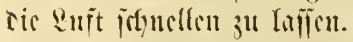

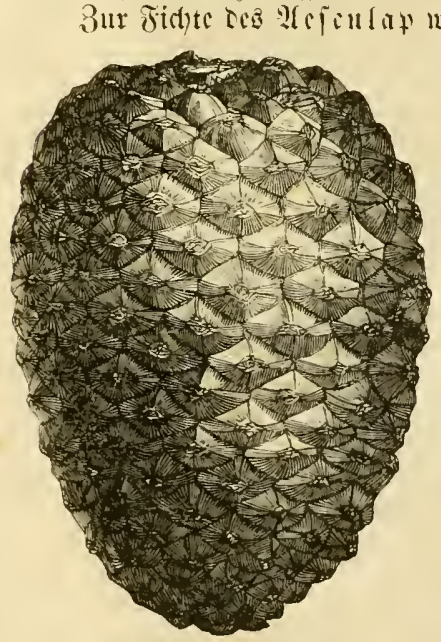

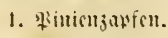

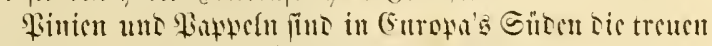

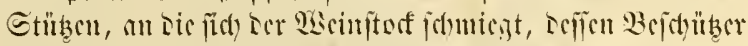

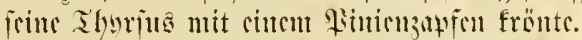

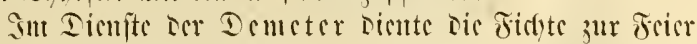
Ler Theamoploricn.

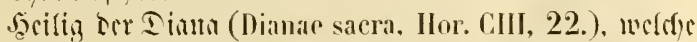

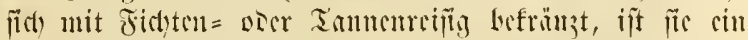

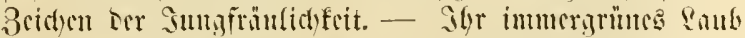

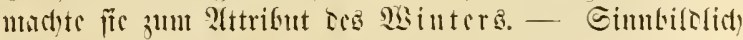

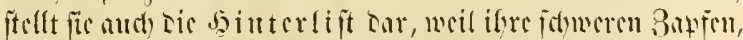

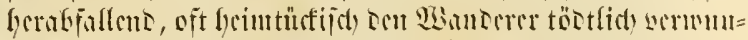

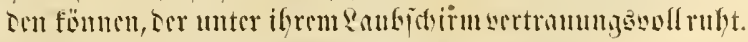

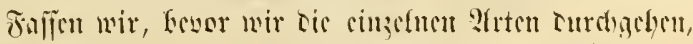

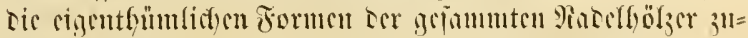
jammen, fo finten wir, tap oor Etumm for bei ma rin=

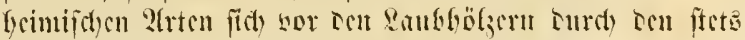

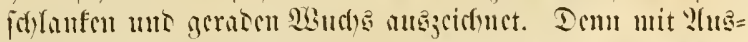

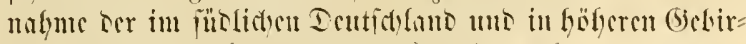

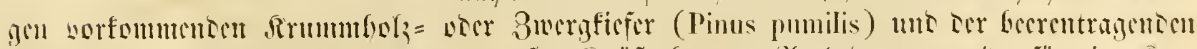

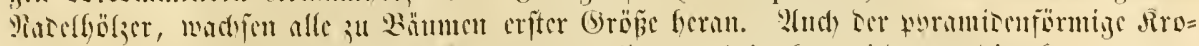

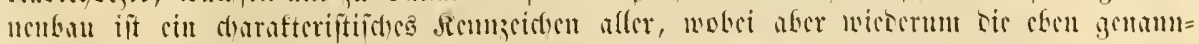

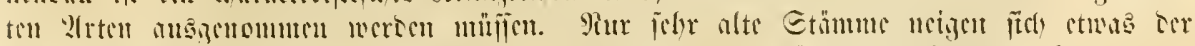

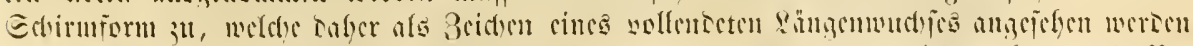

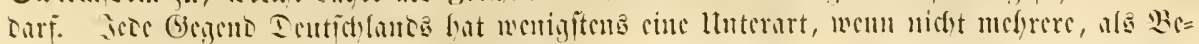

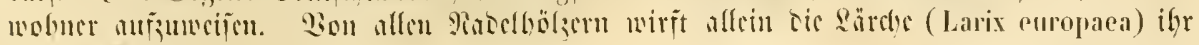

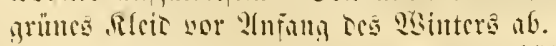

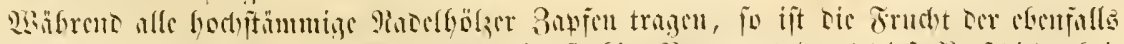

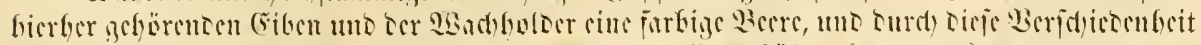

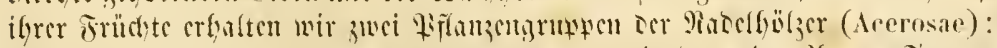

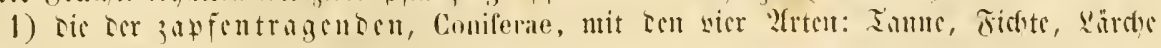

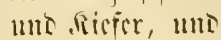

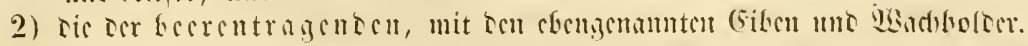




\section{I. $\mathbb{D} \mathfrak{i} \mathbb{\mathfrak { T }} \mathfrak{\mathbb { N }} \| \mathfrak{1}$.}

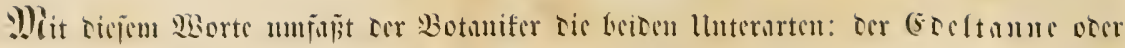

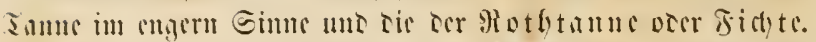

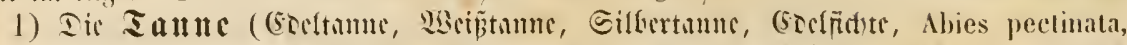

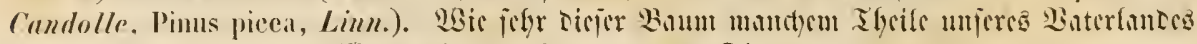

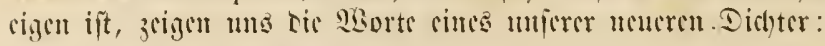

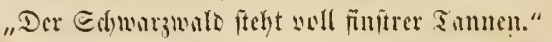

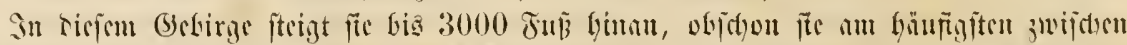

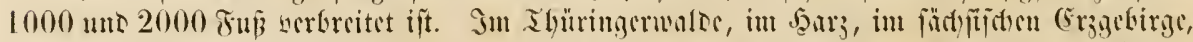

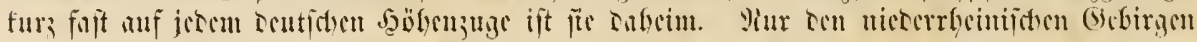

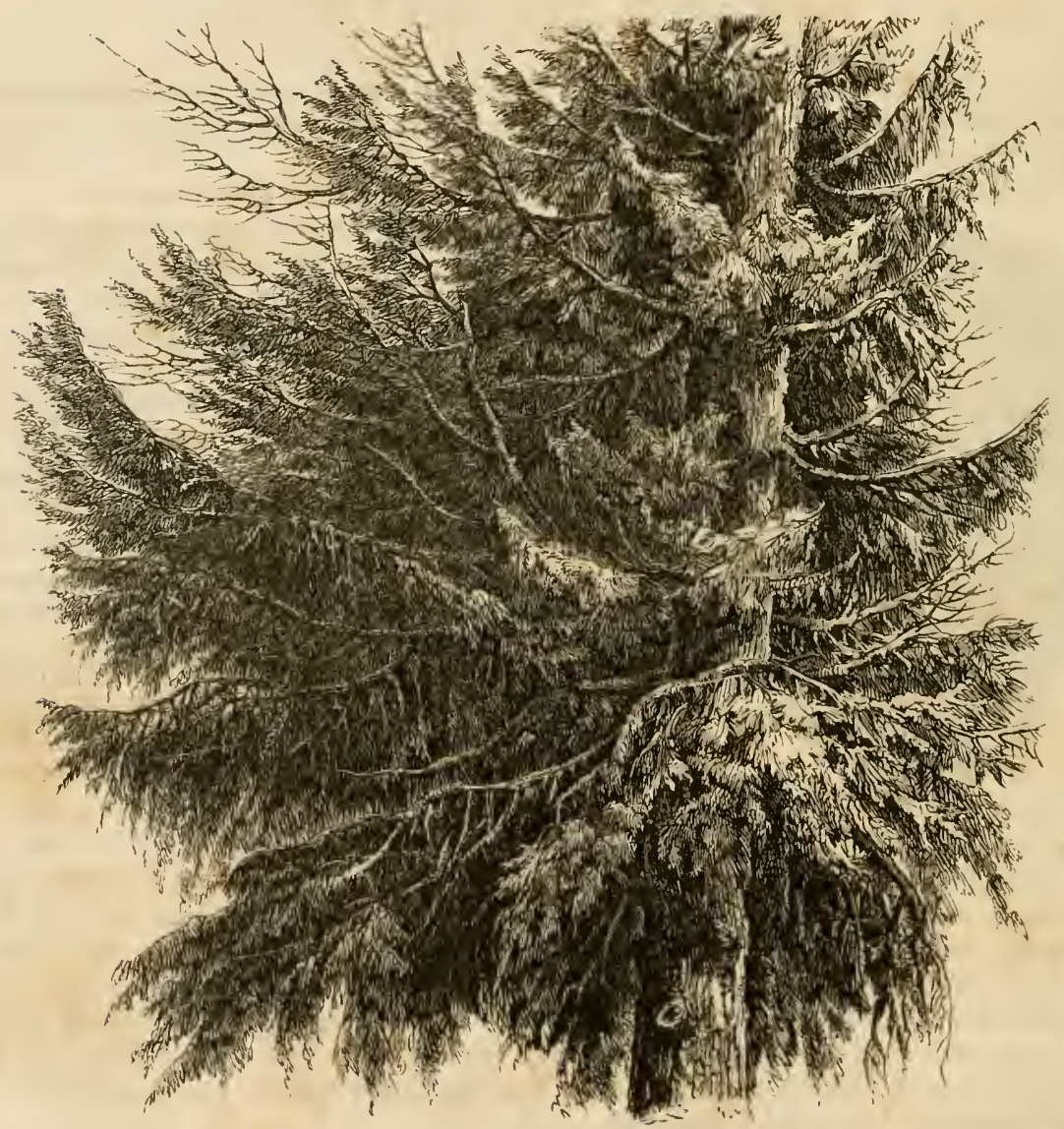

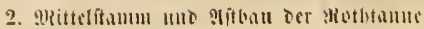

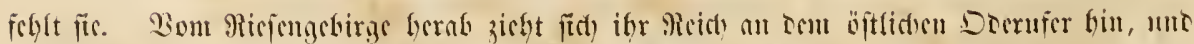

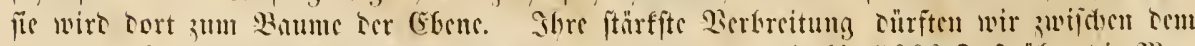

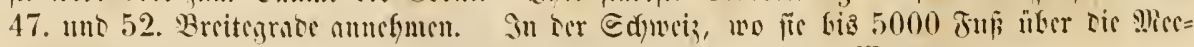

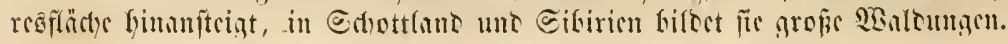




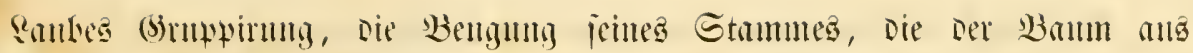

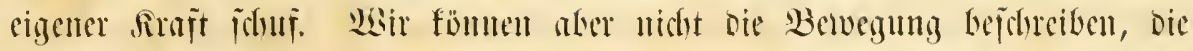

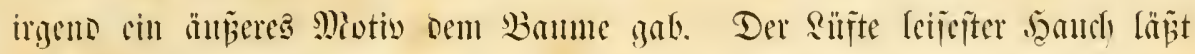
Der Bitterpappel Blätter erbeben; wir hören Die ntyiteriöje Spradic oer

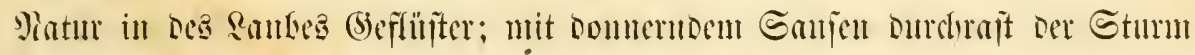

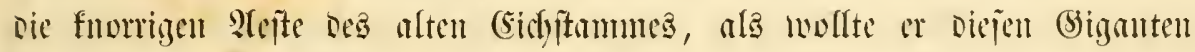

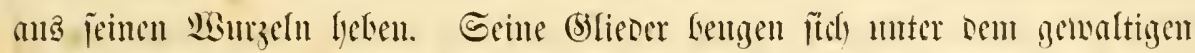

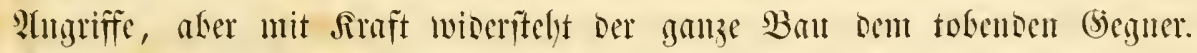

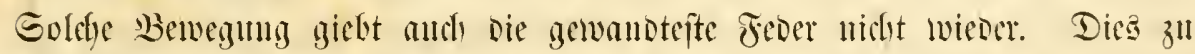

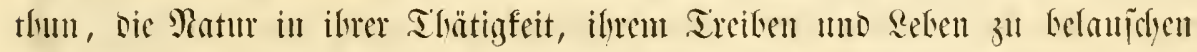

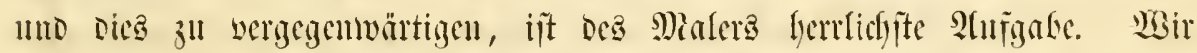

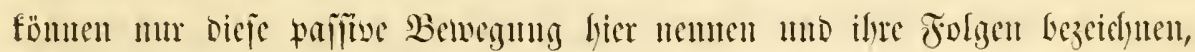

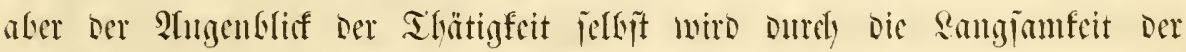
Jorer nidfyt wicorigeigeben.

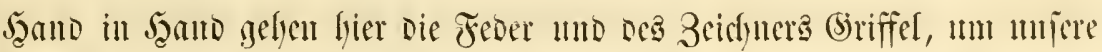

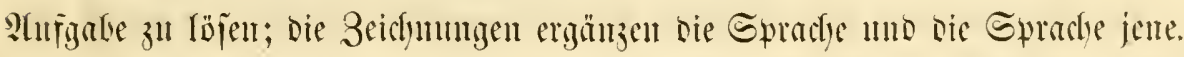

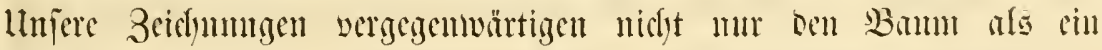

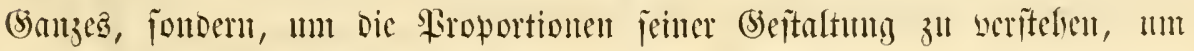

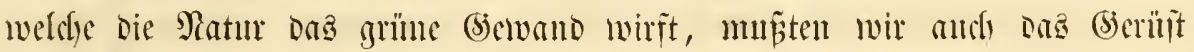

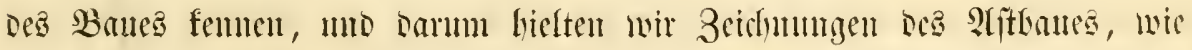

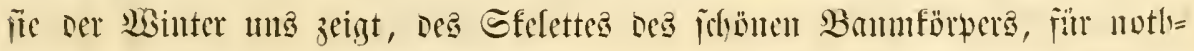

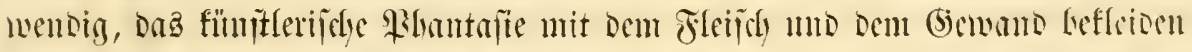

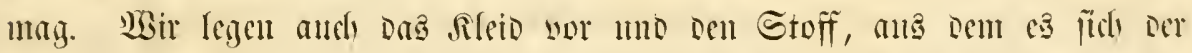

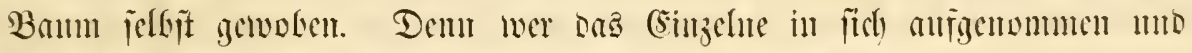

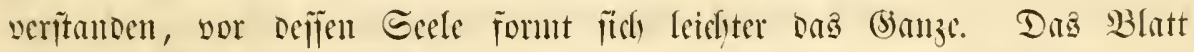

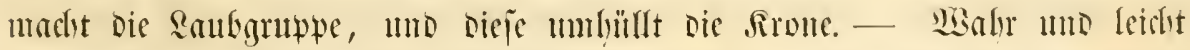

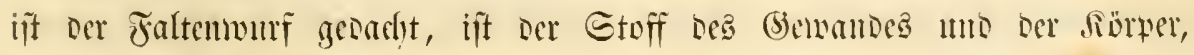
oen es umgiebt, num befannt.

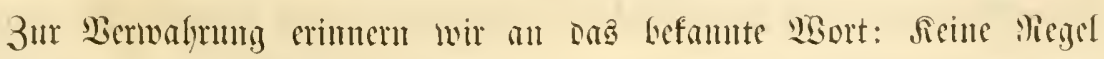

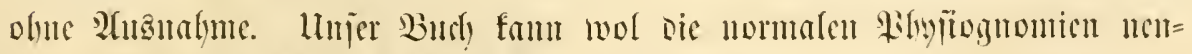

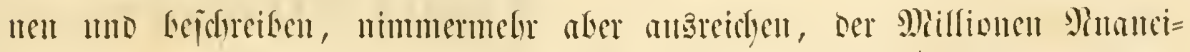
rungen Diejer Inoinionalitäten 3 in geoenfen. 


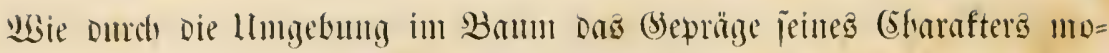

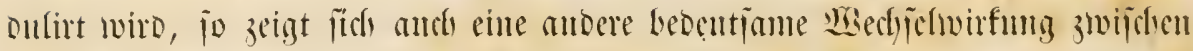
Dem Bamm mo frimer llmgelunis. WBir meiten Des Bammes Etantort. Eime

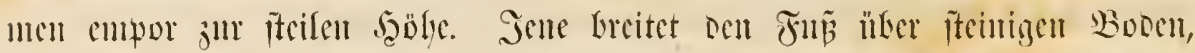

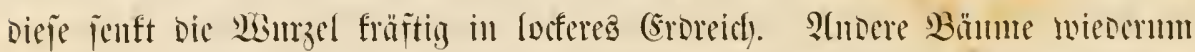

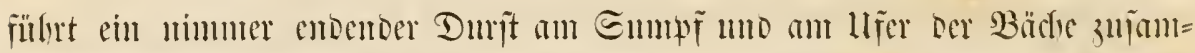

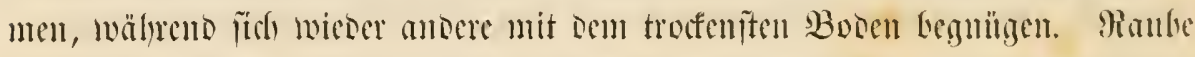

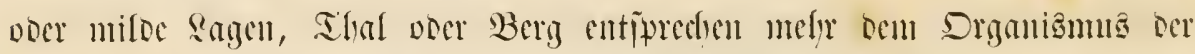

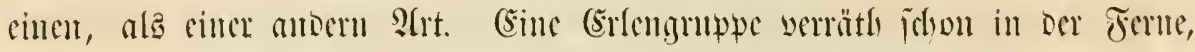

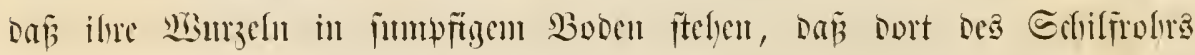

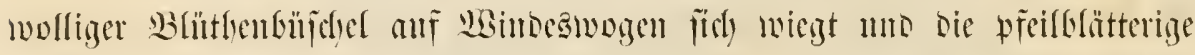

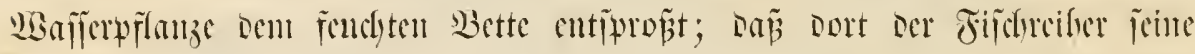

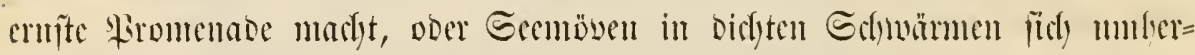

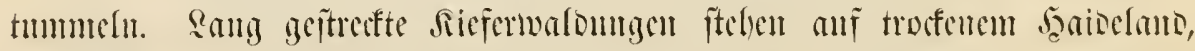

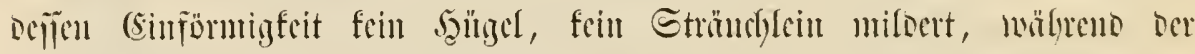

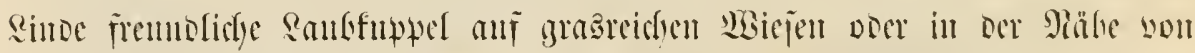

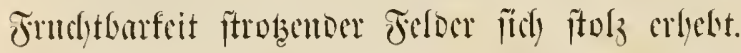

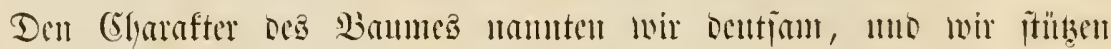

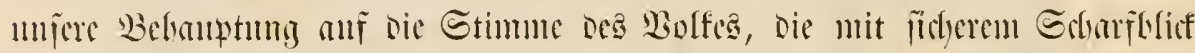

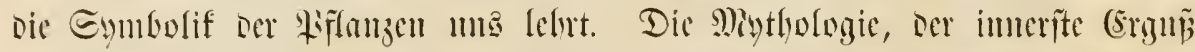

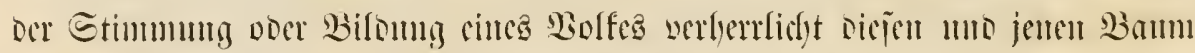

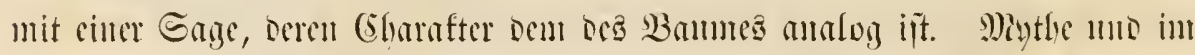

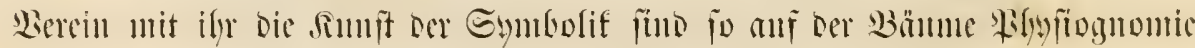

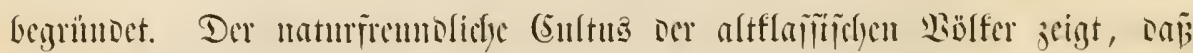

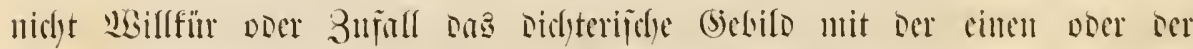

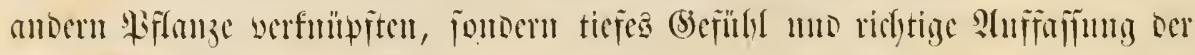

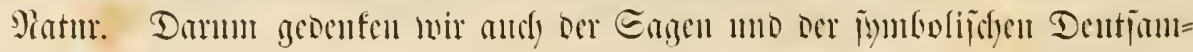

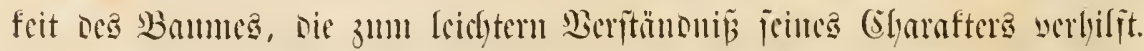




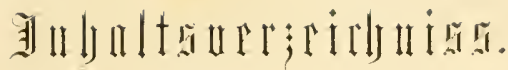

Norunert

Einleitung

Die Madel

i)

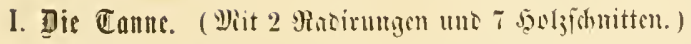

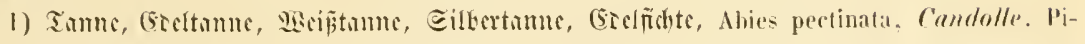
nus picea, Limn.

a. Dic Baliomtanne, Shies halsamea . . . . . . . . . 11

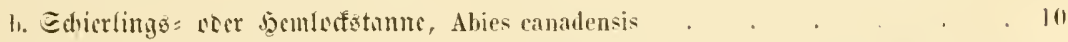

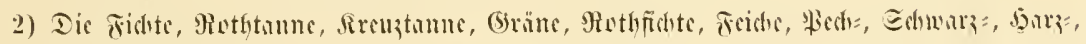
gemcine Tamme, Ahies excelsa, Candolle, Pinus abies. L. weine Fidhte, P. wi. albies alba 10

II. Die Cörd)e. (

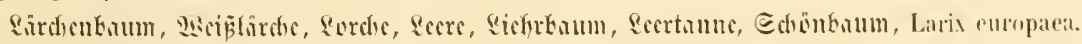

Candolle, Pinus larix, Limn. . . . . . . . . . . . . . 12

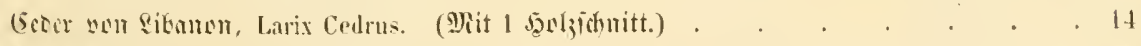

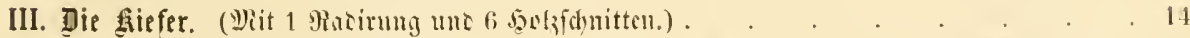

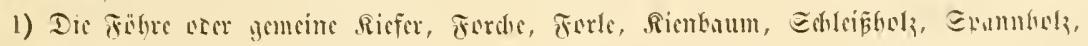

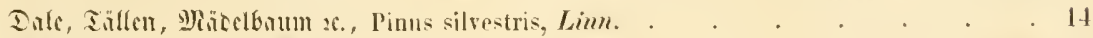

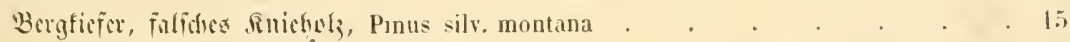

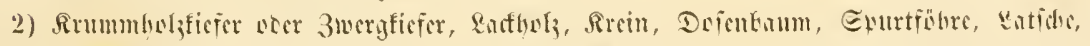
Eecferte, 3ürem u. ה. m., Pinus pumilio, Hänke, P. mughus. Scopl. . . . . It;

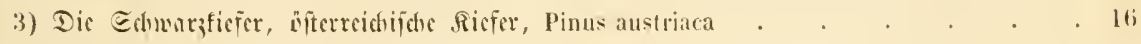

4) Dic 3irbelnufficier, Itre, 3ürme, 3üride, Pinus Cembra . . . . . . 16

5) Die \$gemunuthaficfer, Pinus strobus . . . . . . . . . . . . . 17

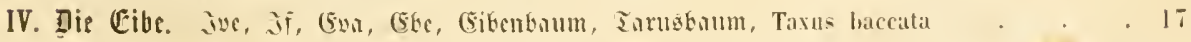

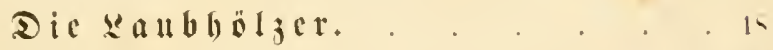

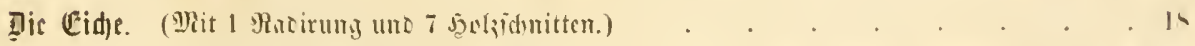

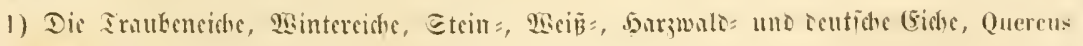
sesilıflora. . . . . . . . . . . . 21

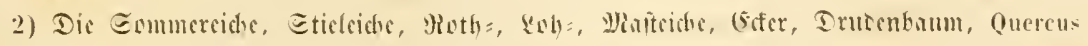
pedunculata, Ehrh., Quercus rohur. Limn. 
3) Dic Edmunzeidle wer meidhanrige Eiche, Quercus pubescens . 25

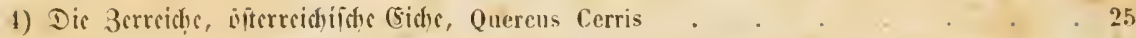

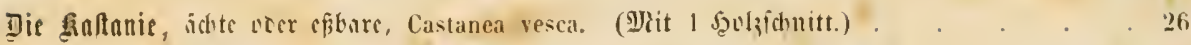

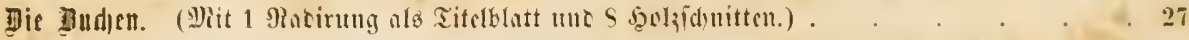

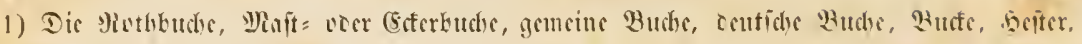

Fagus silvatica, Lim. . . . . . . . . . . . . 27

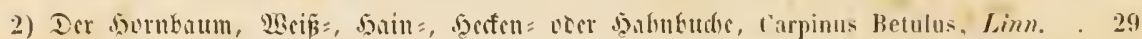

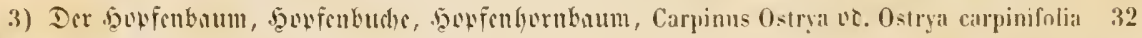

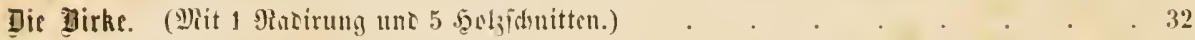

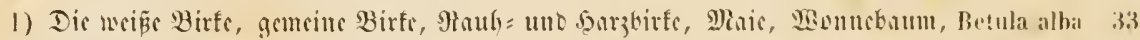

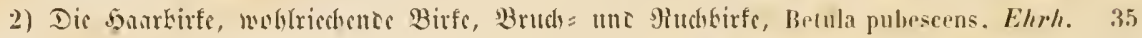

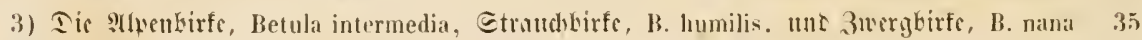

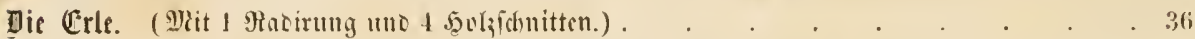

1) Die Edwurzeller, Motherle, Erte, Elie, Elfern, Sller, Dleer, Sller, Urle, Alnus glutinosit. Gärtner. Betula Alnus, Limm. . . . . . . . . . . 36i

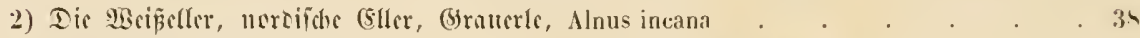

3) Slfwencller, Drullel, Bergerullel, Alnus ovala veer Aln. viridis . . . . . . 39

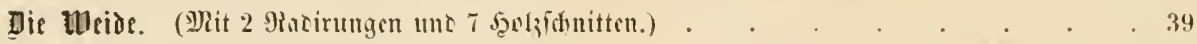

1) Dic Evhl= voor Sanlircioc, Salix caprea . . . . . . . . . . 41

2) Die grof́blïttrige Wiscioc, Salix grandifolia . . . . . . . . . 42

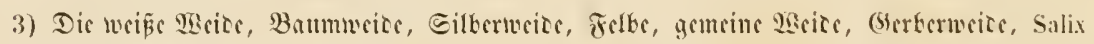
albal, Limt. . . . . . . . . . . . . . . 42

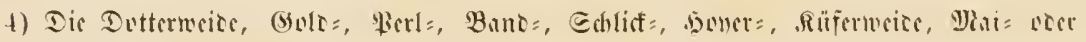
infubaguly, Salix vitellina. . . . . . . . . . . . . . 43

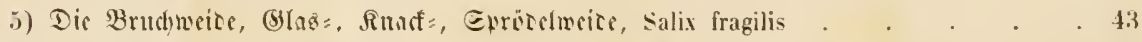

6) Dic fraume 2Escice, Salix Russeliana . . . . . . . . . . . . . . 4.3

7) Dic kreitblättrige gierioe, Salix Meyeriana . . . . . . . . . . 4.3

५) Dis entbermeite, Salix pentandra . . . . . . . . . . . . 4.3

9) Dic Trthlyecise, Salix praecox . . . . . . . . . . . . . 13

10) Sic Trumer = ner bangeweitoe, Salix babilonica . . . . . . . . 14

11) Dic Ealferimcitc, Salix aurita . . . . . . . . . . . 4

12) Die Sanarucice, Salix aquatica socr S. cinerea. Lime. . . . . . . . . H

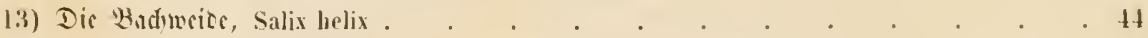

14) Dir Rothyecioc, Salix rubra . . . . . . . . . . . 44

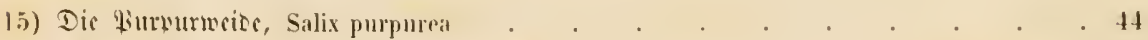

11) Dic Rorburcioc, Salix viminalis . . . . . . . . . . . . . 4t

17) Dic Rrebamcice, Salix triandta. . . . . . . . . . . 4

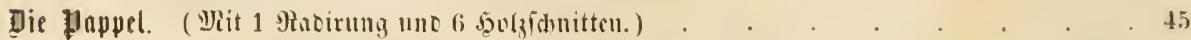

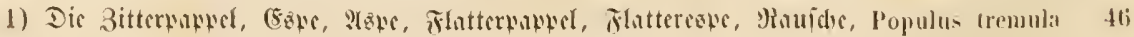

2) Dir Eilferwatheln, Tomentosile. . . . . . . . . . . . 4s

it. Sic Silberbintel, Populus alba . . . . . . . . . . th

h. Dii grutti linuwel, Populu canescens. . . . . . . th 
Tic Edmarituffulu, Marginalae

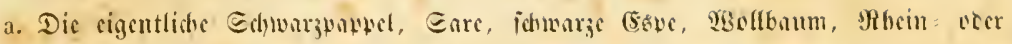
butpelucise, Populus nigra

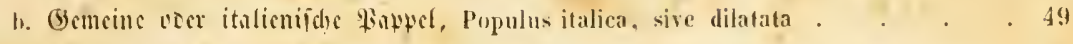

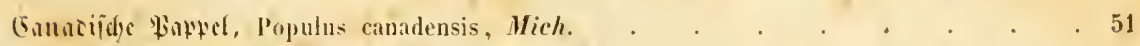

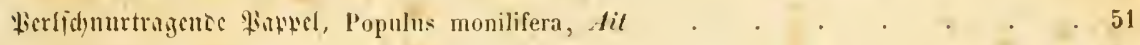

Buliampityel, Populas balsamiferat, Linn. . . . . . . . . . . . . 51

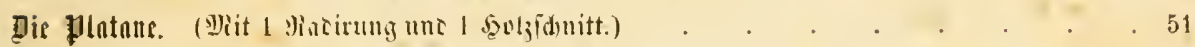

1) Dif morgentäncific YShatane, Platanus orientalis . . . . . . . . . . 52

2) Dri atenclintilde Pifatanc, Platanus occidentalis . . . . . . . . . 52

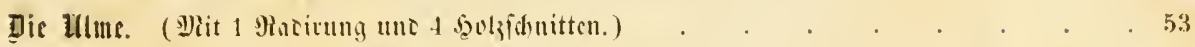

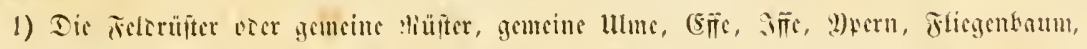

l'Imus compestris . . . . . . . . . . . . . . . . . . . 54

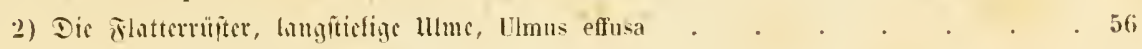

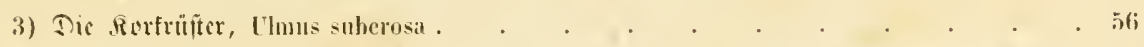

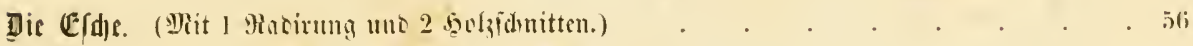

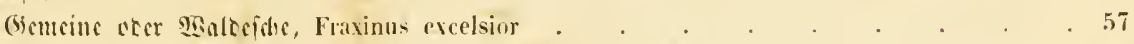

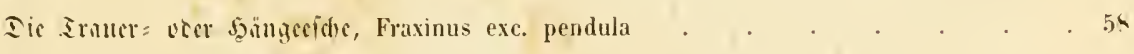

Fic frimeflattrige (Sidic, Fraximus crispa . . . . . . . . . . . . . . 59

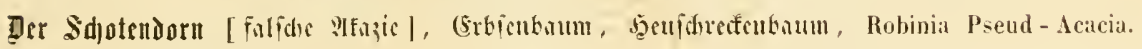

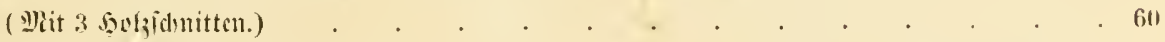

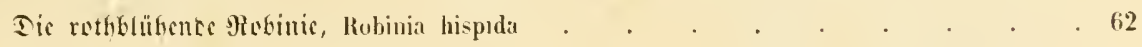

Tic liedrafisic, Robinia viscosil . . . . . . . . . . . . . . 62

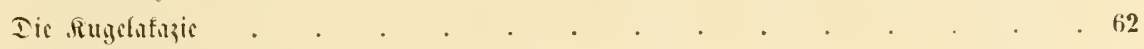

Tur Färbeginter Genista linctoria . . . . . . . . . . . . . . 62

Der Bsoloreyen, gelbe stfazic, Rlecbum, Cytisus Labmenum . . . . . . 62

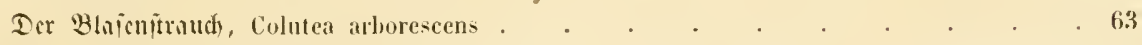

Ier Binientricmen, Spartiun juncenm . . . . . . . . . . . . . . . 63

Dor Eted)piricmen, Genista germanica . . . . . . . . . . . . . . . . 63

Fic 5aubedelarten, Ononis spinosa unc 0. repens. . . . . . . . . . ti.3

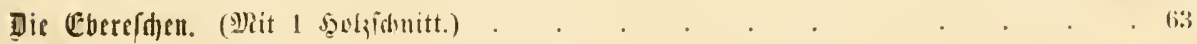

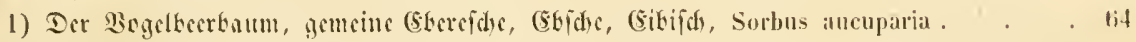

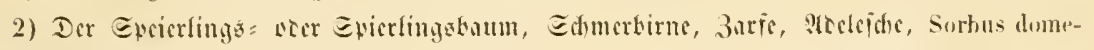
slica, Limn. . . . . . . . . . . . . . . . . till th t

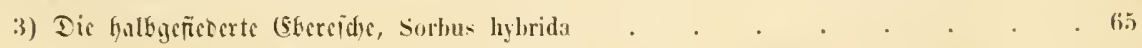

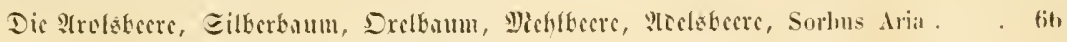

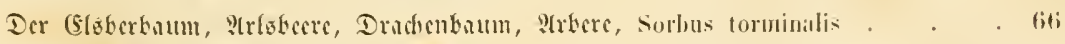

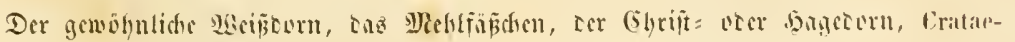

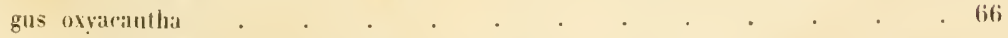

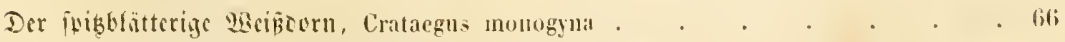

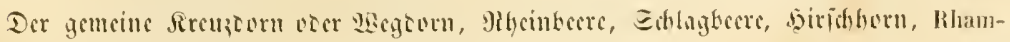
nus catharticus . . . . . . . . . . . . . . . . . . . . . . .

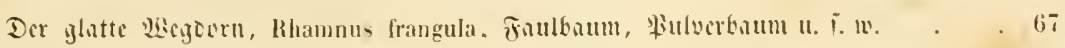


Der Plyemegeren, Rhamnus alpinus

Itr 5ुMclitriudb), Coryllus avellana

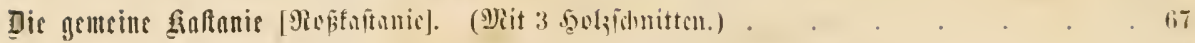

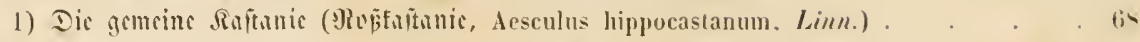

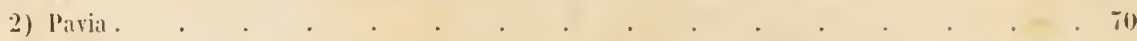

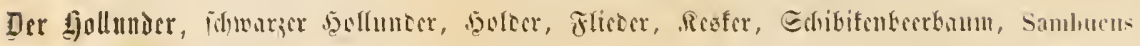

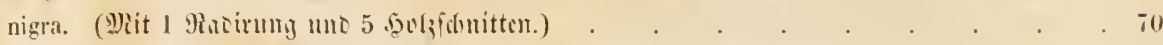

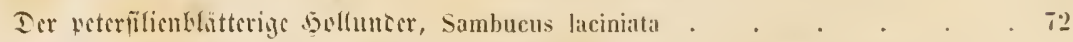

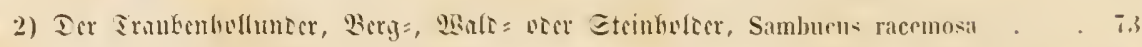

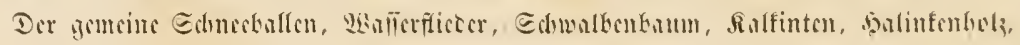

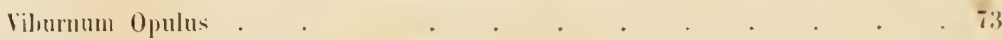

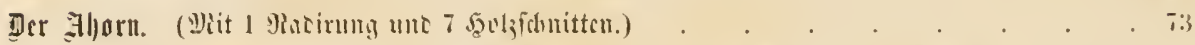

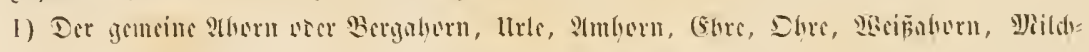

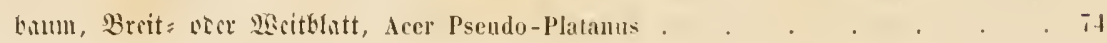

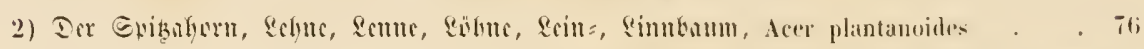

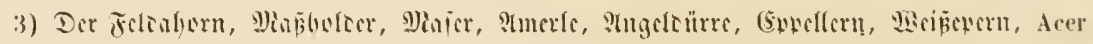
campestre . . . . . . . . . . . . . . . . . . . . . . . .

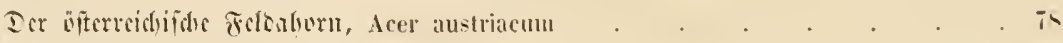

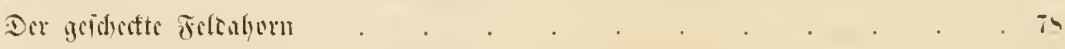

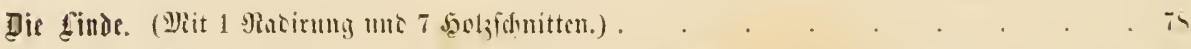

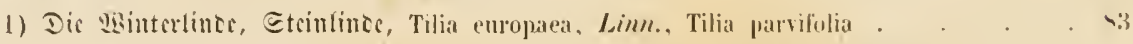

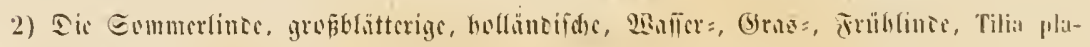

typleyllat verer grandilolia . . . . . . . . . . . . . . . . . . . . . . 


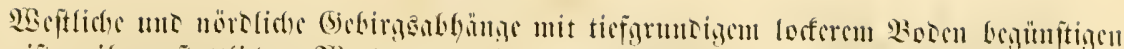

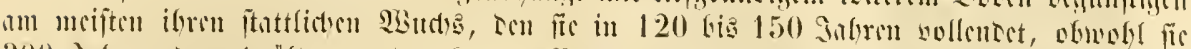

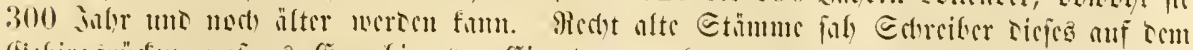

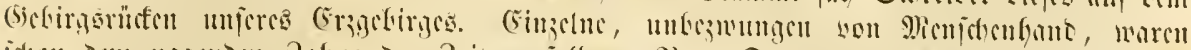

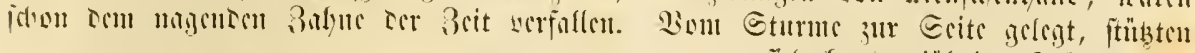

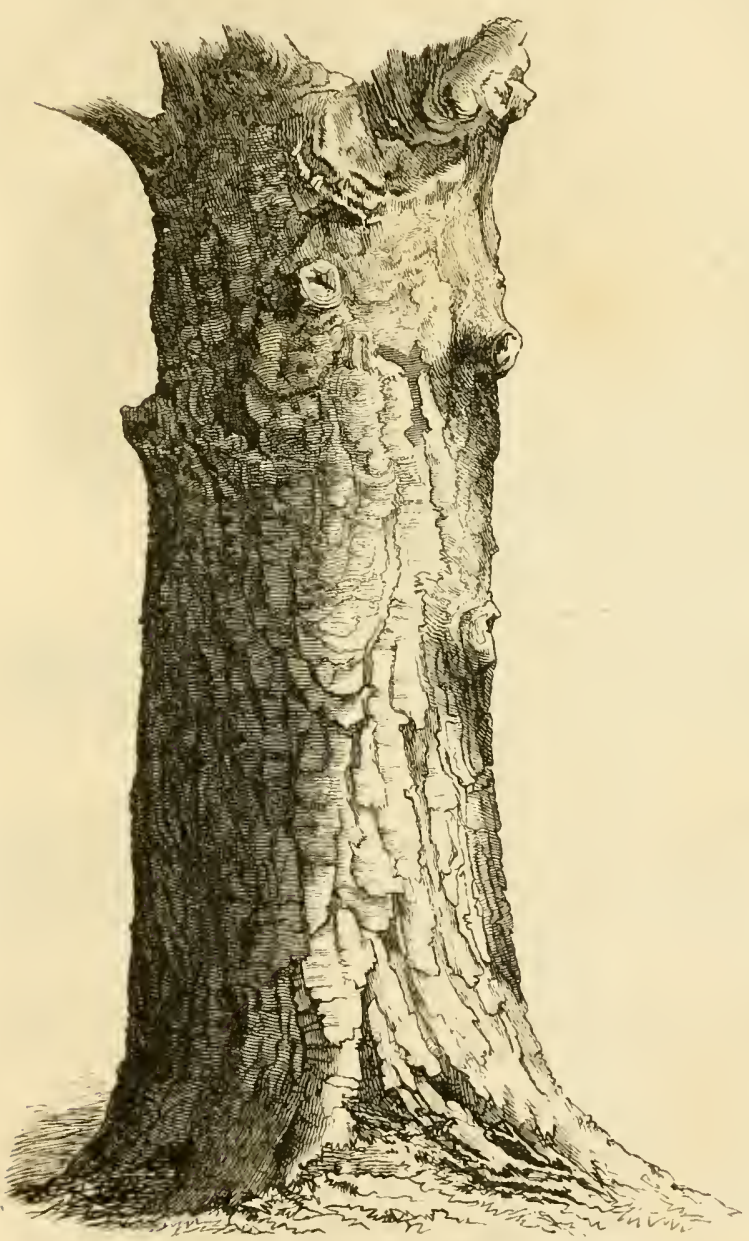

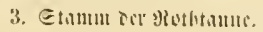

ïs) Lunertjübrige Etimme auf Lic Edoultern tor frifitizern in= gent. Eason batte auf fer obern, tou Şimmel zugemanten Erite

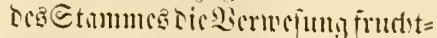
brimgenten 2 sosm gebilest, in tom

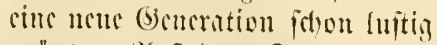

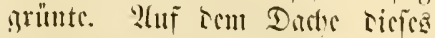

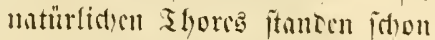

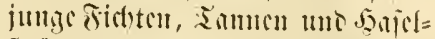
ftrïuder, uno jo (rfluto er fide, wis

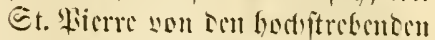

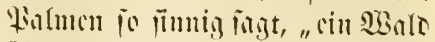

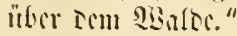

Ilnter günittịgen llmitümsen cr=

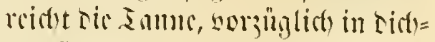

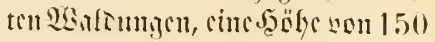
Fui mit riner Gtanfe vou 12 Fui;

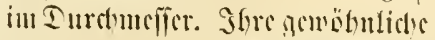
Jöbe ift bei $3-4$ Fuis Iurdt= แ11กเx 100 bis 120 Fur. In ifyren erften 50 sulfren ift ibr

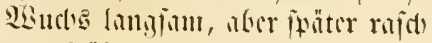
unt frifftisy.

Shr geritser, waljenförmiger

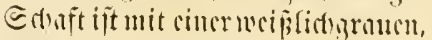
menis rijizyest Rinse feffericet, mocurd fir fïd bejonters non tor

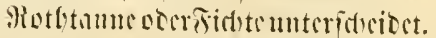

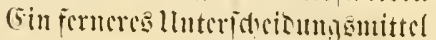

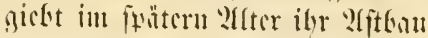
aII ris 5 anto, intem ric fojt im redten Disinfel wom Etrmume at ftebenten ?lepte nath mujen bin

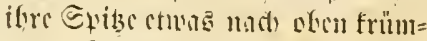
men. Eic bifren sine tidnte iturizis Sinrmmioe, tic bei im Errien er=

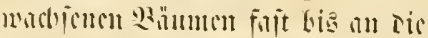

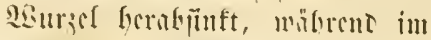

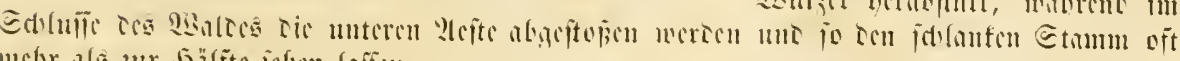

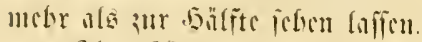

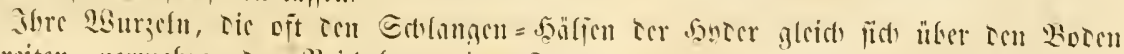

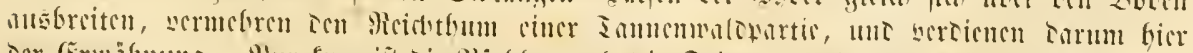

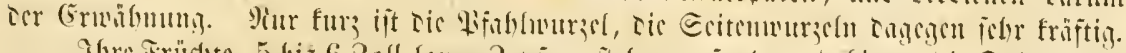

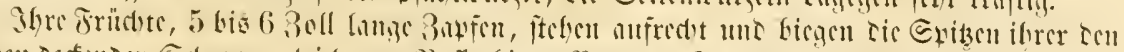

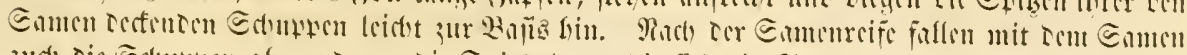

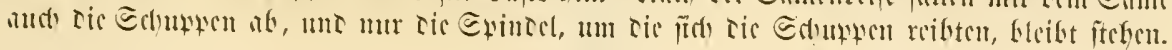




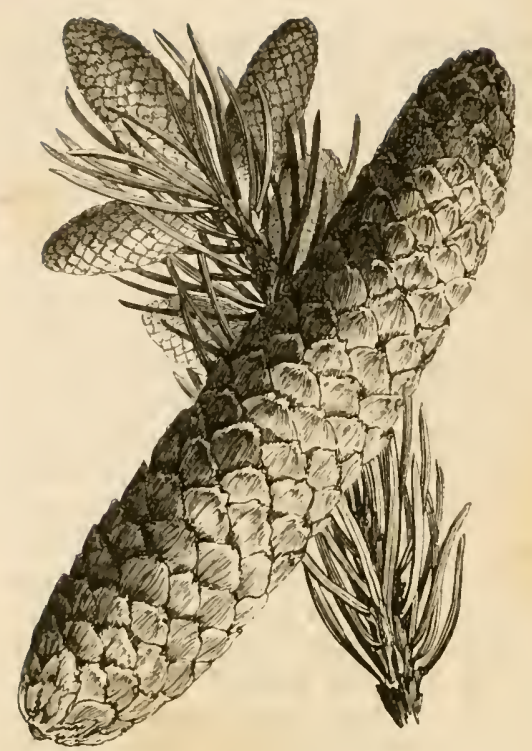

1. 3aพren Der Bothtaunc.

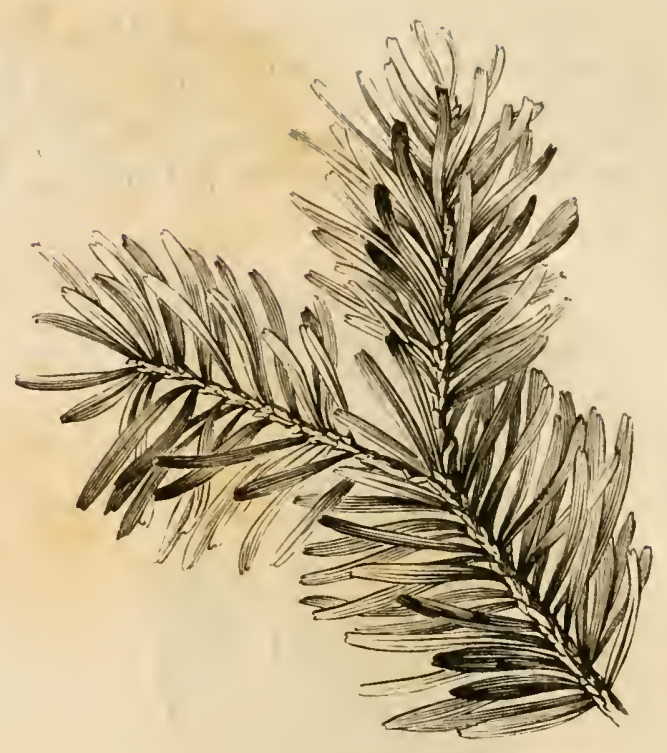

5. Matelítuit der Edeltnume.

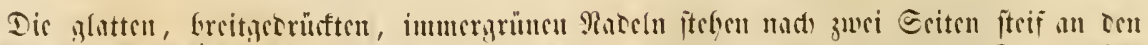

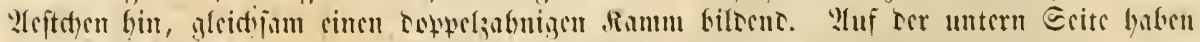

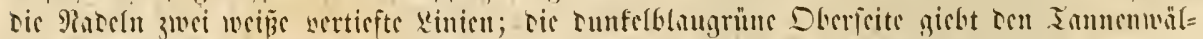

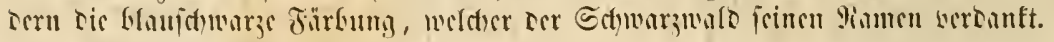

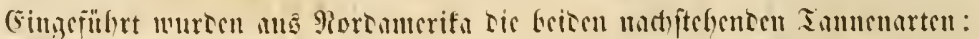

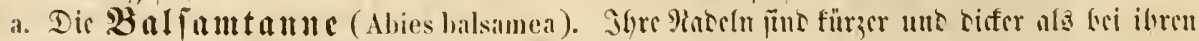

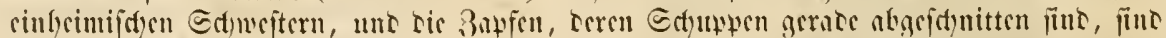

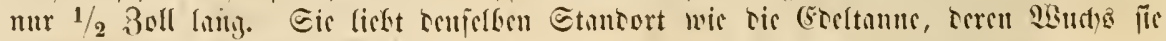
(and) biat.

Rod) fleiner fint tic Bapfen tor

b. Schicrling

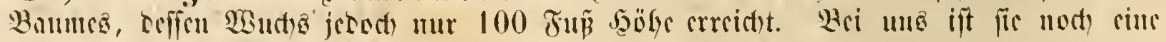
irltene (5ulturpiflumje.

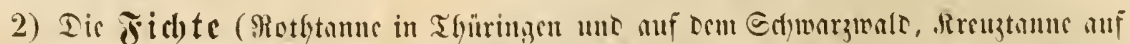

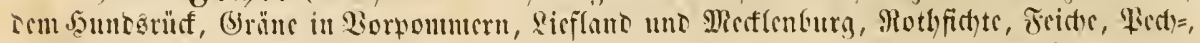
E(f)war $=$, Sar $;=$, gcurciuc Tanuc, Abies excelsa, Candolle, Pinus abies, Linn.).

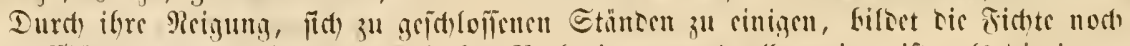

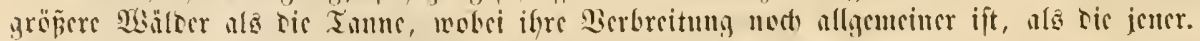

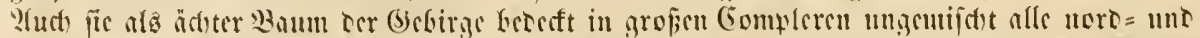

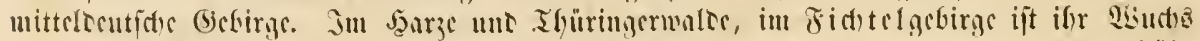

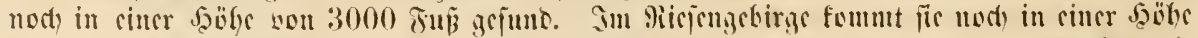

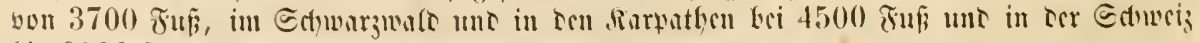

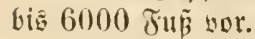

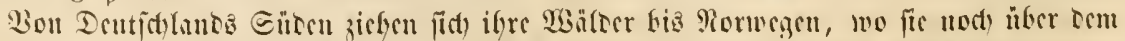

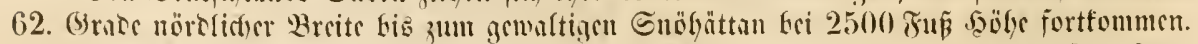

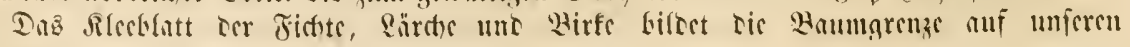
(i)bingen. 


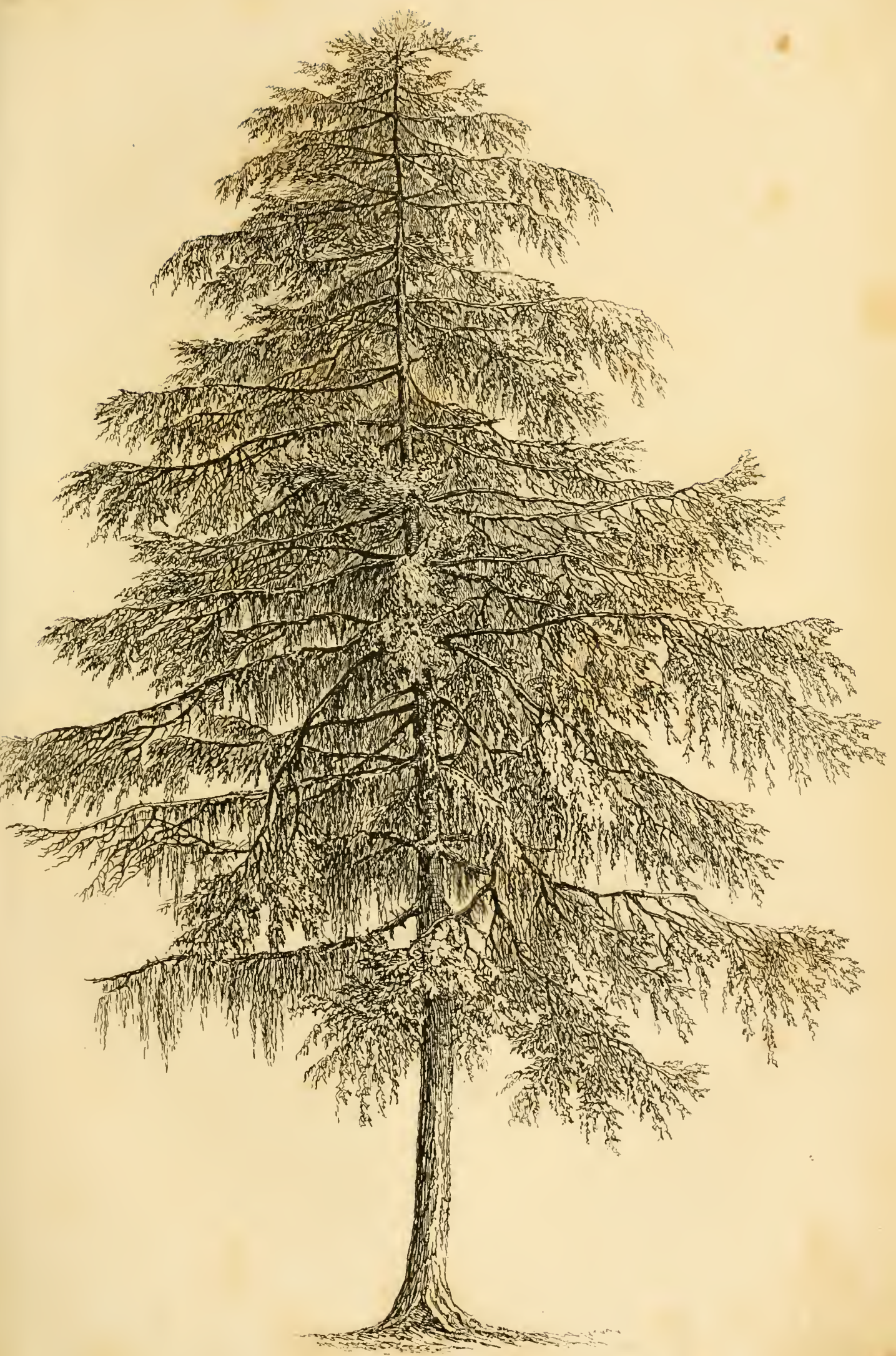





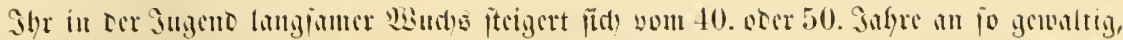

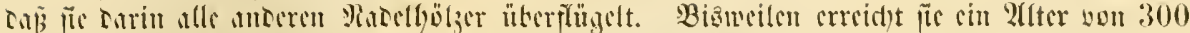

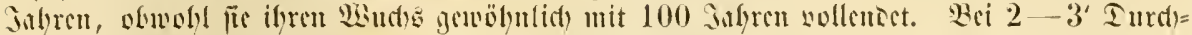

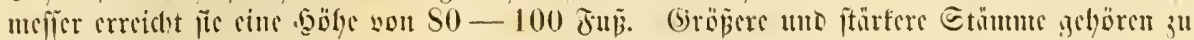
in jeltencu.

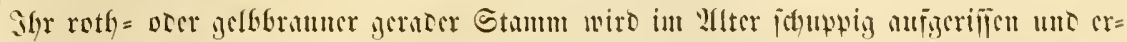

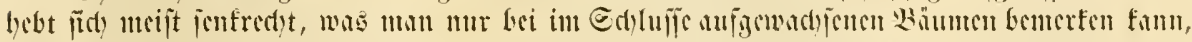

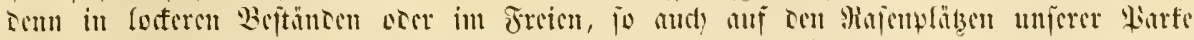

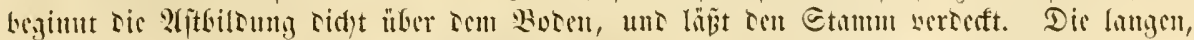

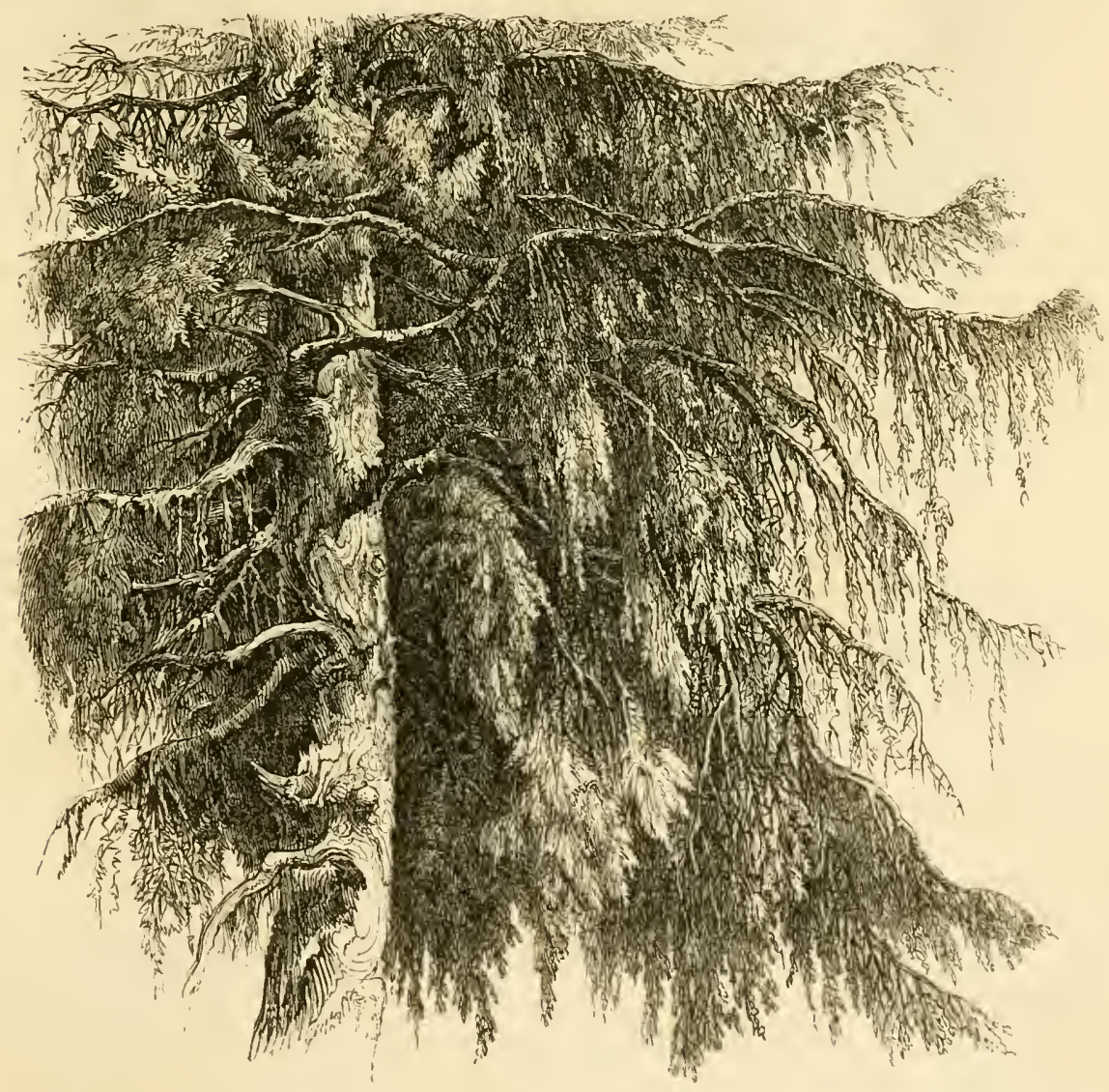

6. Stitbu rer jitifte.

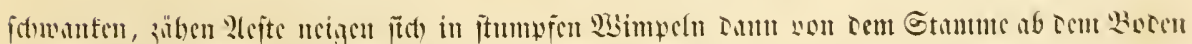

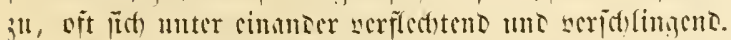

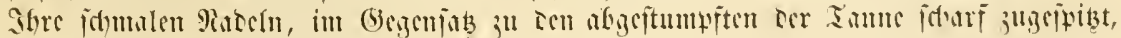

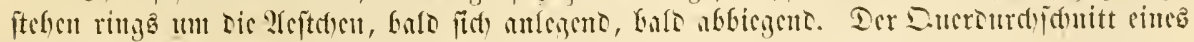

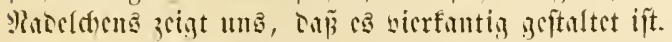

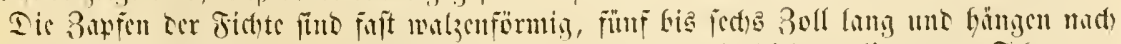

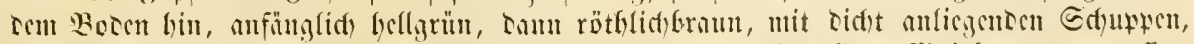

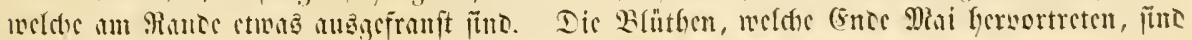

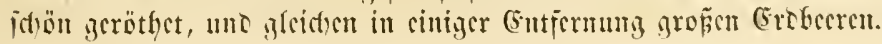




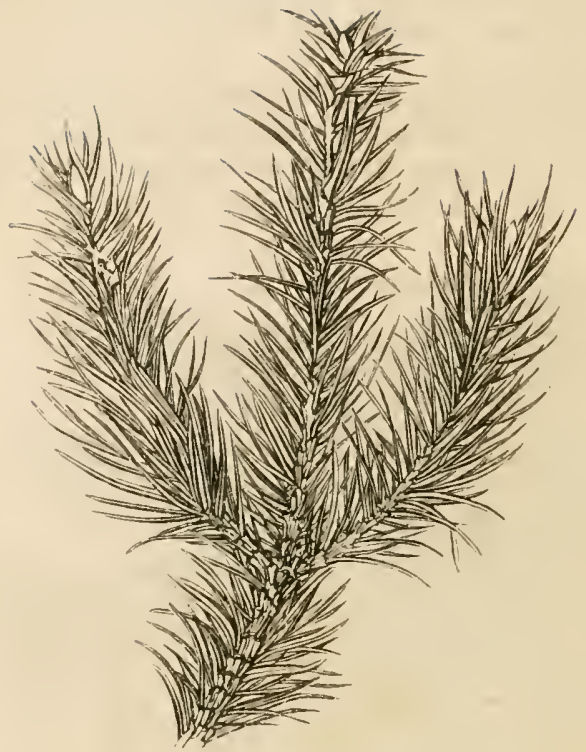

7. Madelitum ser sidite.

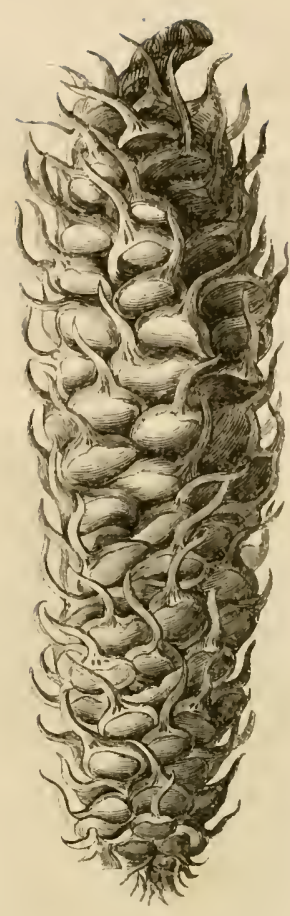

8. 3awfen int Jidfte

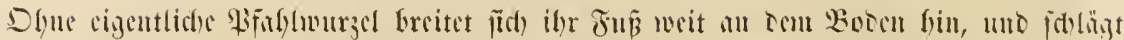

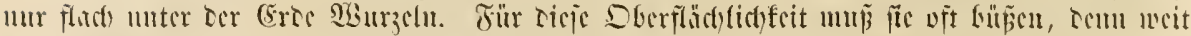

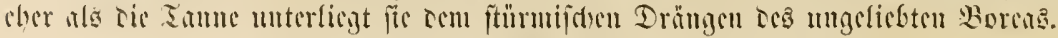

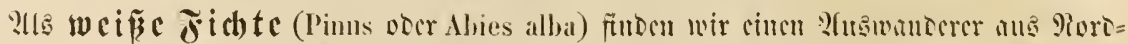

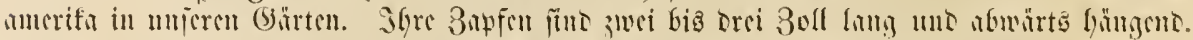

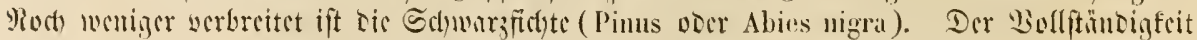

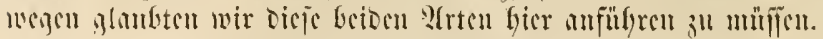

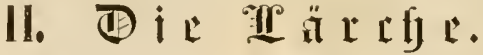

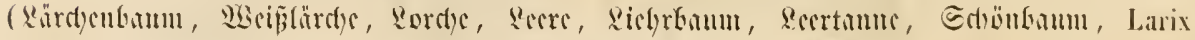
europaea, C'andolle. Pinus lirix, Limn.)

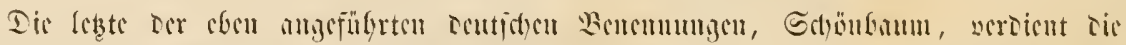

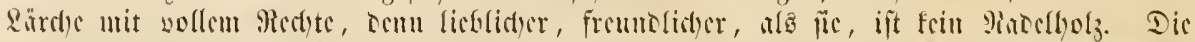

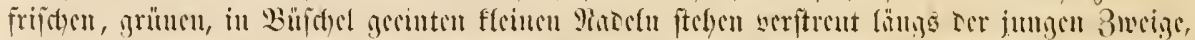

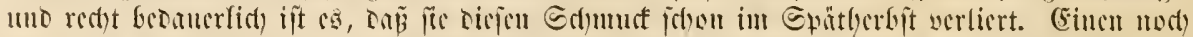

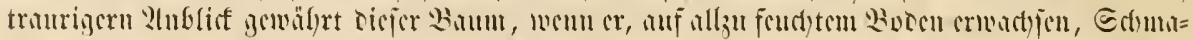

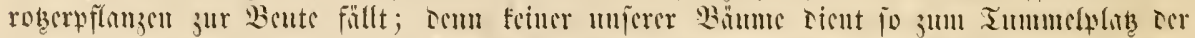

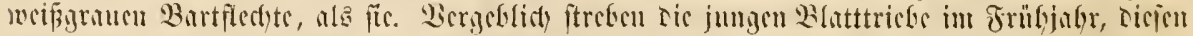

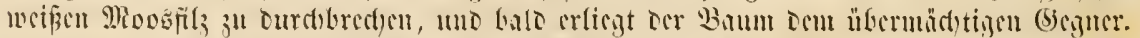

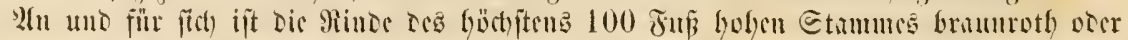

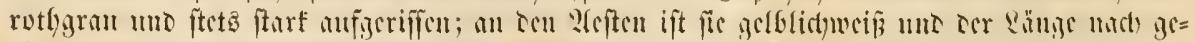

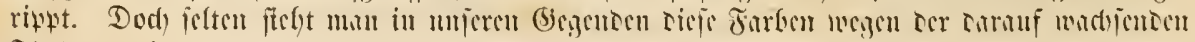
Flledten rein. 


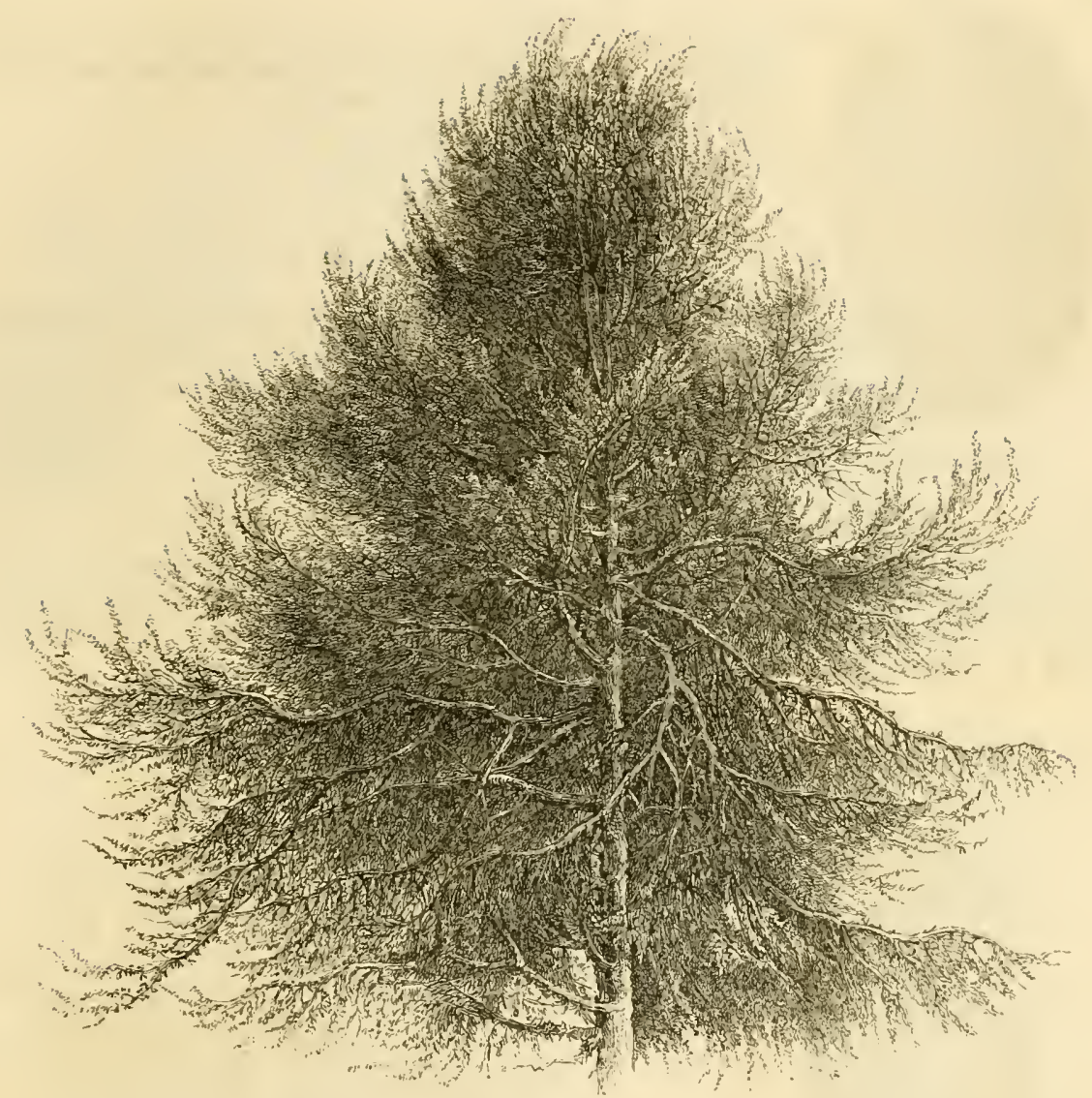

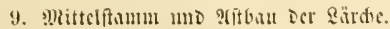

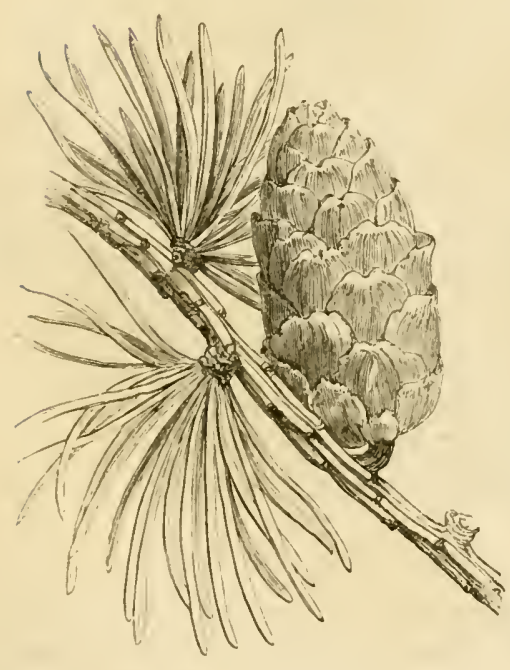

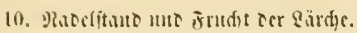

Die Sronc Des Rärd)enbamm ift chonfalls cinc

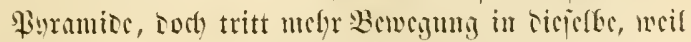

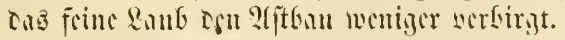

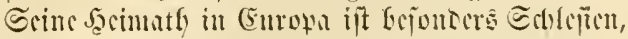

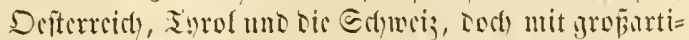
gem llebergemid)t gezen antere Bambarten tritt or in

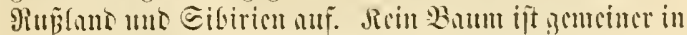

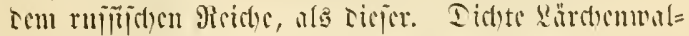

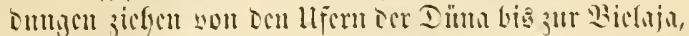

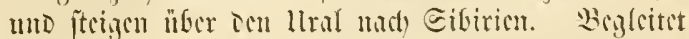

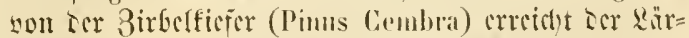

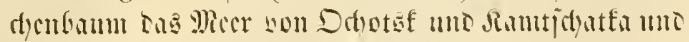

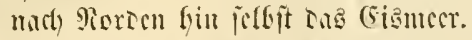

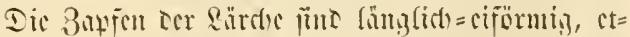

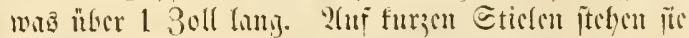

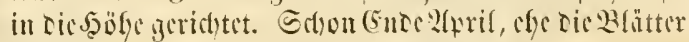

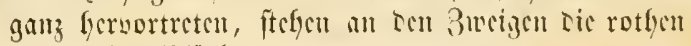
uno gelfon $\mathfrak{B l u ̈ t}$ yon. 


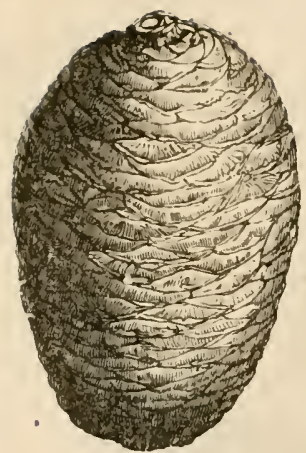

11. ซิrudit ter (5eter.

3u Der (5attung karthe wiro and die (Eeder von Qiba=

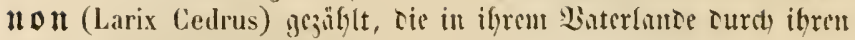

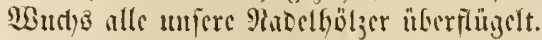

\section{Dî $\mathfrak{E}$ li $\mathfrak{i} \mathfrak{e} \mathfrak{f} \mathfrak{e}$.}

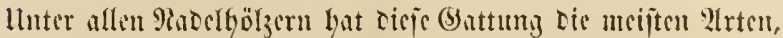
nout tenen allein fünf in umierem Biaterlante sorfonmen.

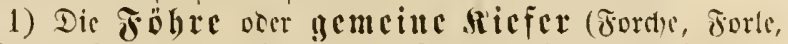

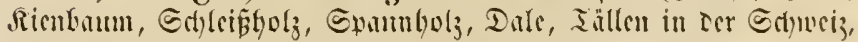

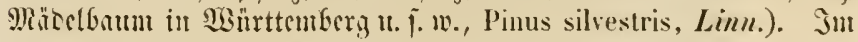

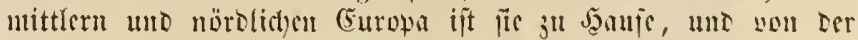

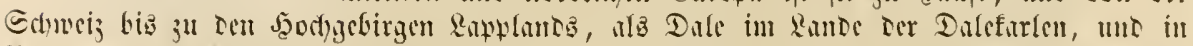

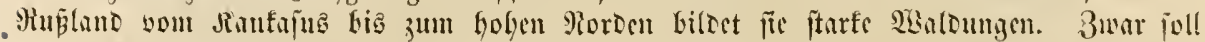

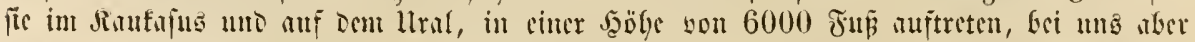

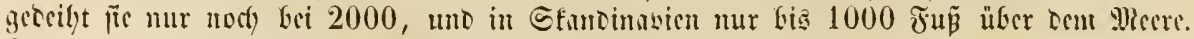
Dafer ijt lelumiger Smoboren ilge liebiter :Lufentfalt.

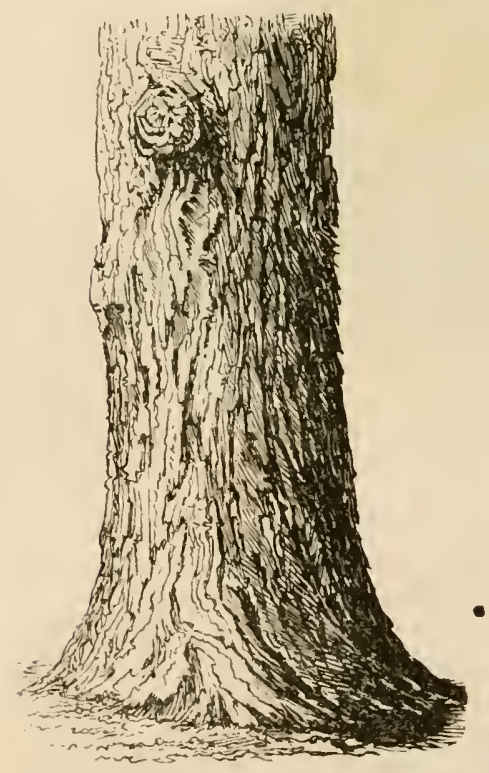

12. Sicferfaมmแ.

(Sine rifinge rothbraune Rinde ume büllt ifren Strum, orr fidt) oft f(t)ou

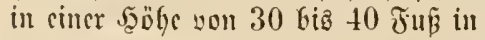
methere Bepte foultet. Se mathoem ifre icr Stumbort gümitig, vollentost ite

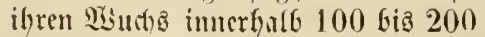
Sufgrer. Dis Sirone ift in Der Sugeno pyrantionl.

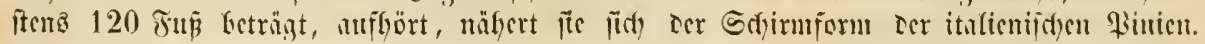




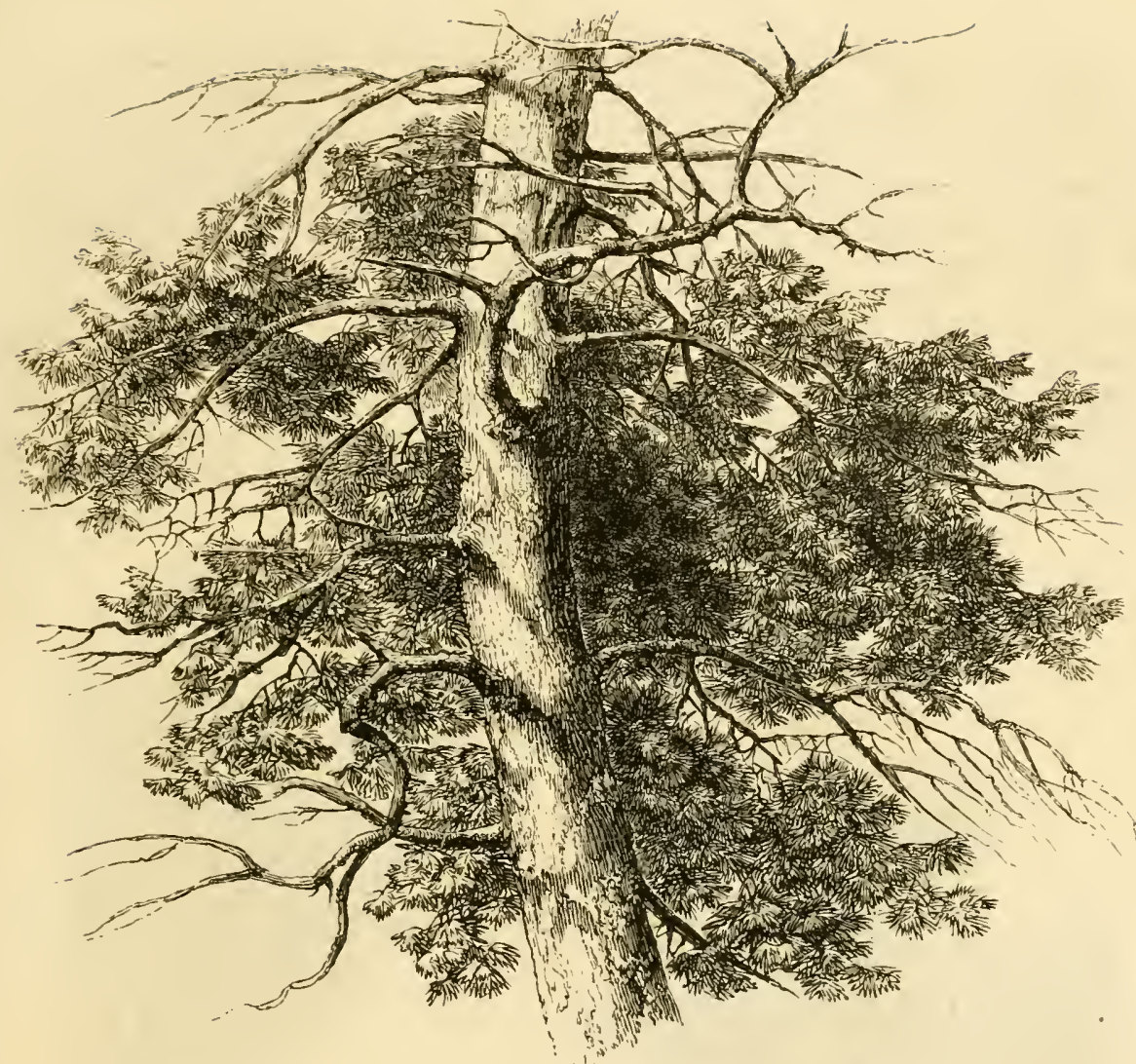

14. Mittelitanun ter sisfier.

Dis fïrferen Scite, ebenfalls rothbraun wic ocr Etamm, ftreben quirlitänoig, of̣t verfrüppelt, nacb) allen Ridftungen, uno bilten cinen breiten 2) fิtratiug.

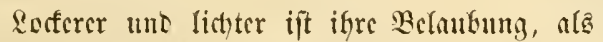

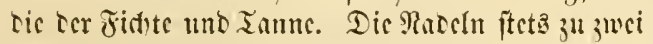
oter trei in einer @etucite, fint zueci bis orei 3oll lang unt ant Rante ter Ränģe nat) umgejct)lagen,

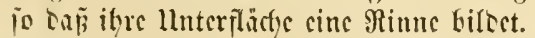

Dic ciförnigen, jugciptzeten Bałfen Gänizen berab unt juno mit abucitumpiten, oben crbabenen ङ (scite.)

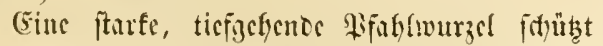
fie sor Der Jainfälligfeit ilyrer anderen Familien= glicoer.

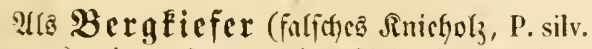
montana) tritt unjere gemeine Ricjer in fnorriger, werfrüppelter (Seftult mit nicterbangenten 3 weigen แuF boben, fumprigen Beriglagen auf.

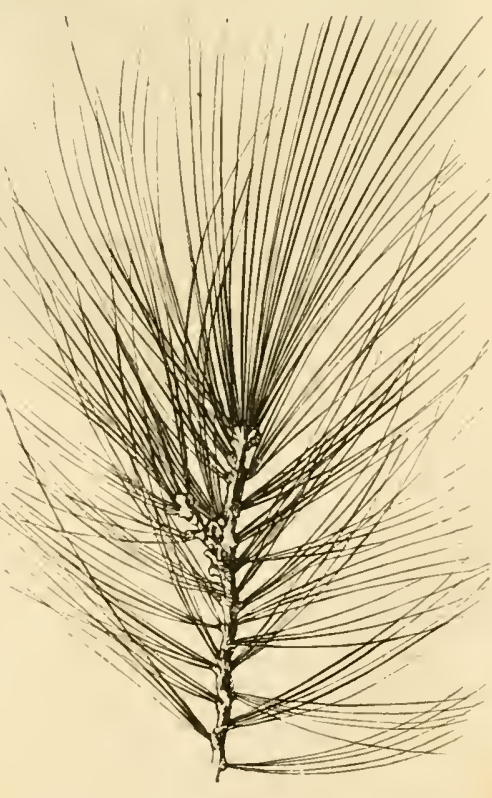

15. Mardu oct sicfer. 


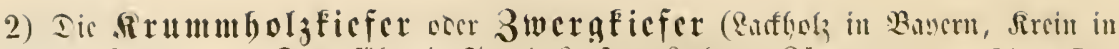

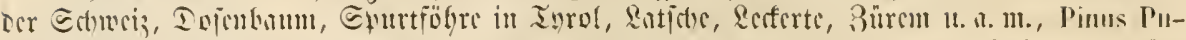

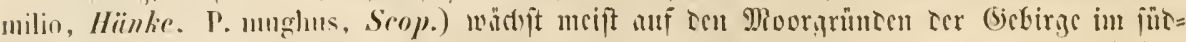

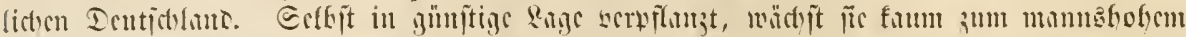

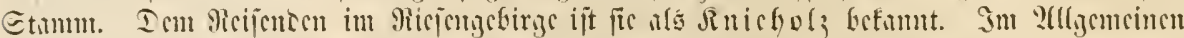

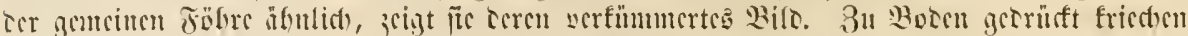

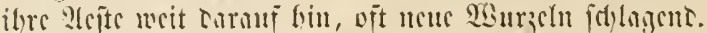

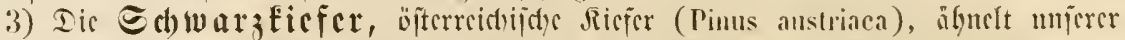

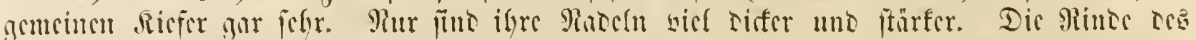

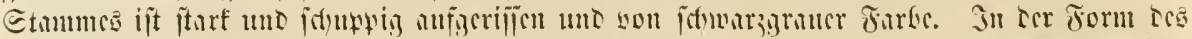

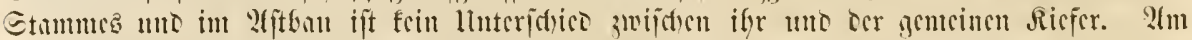

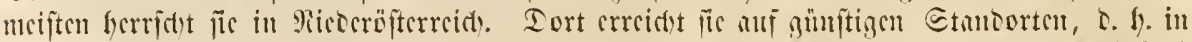

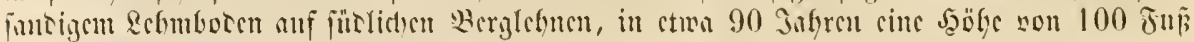

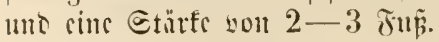

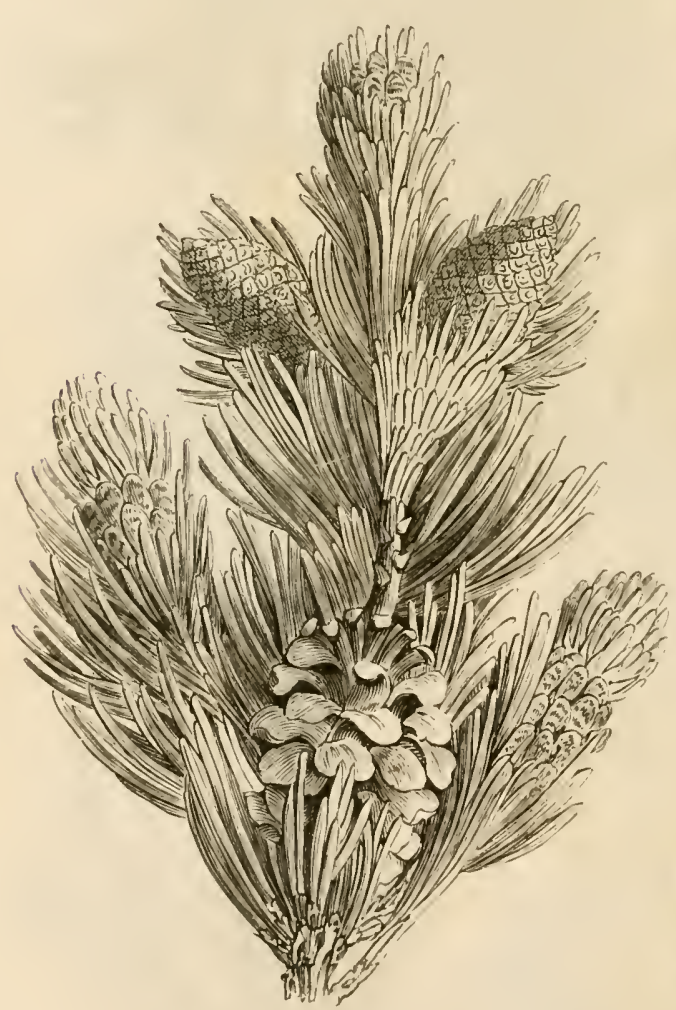

1ti. frtmonoly: socr 3 werpfoficr.

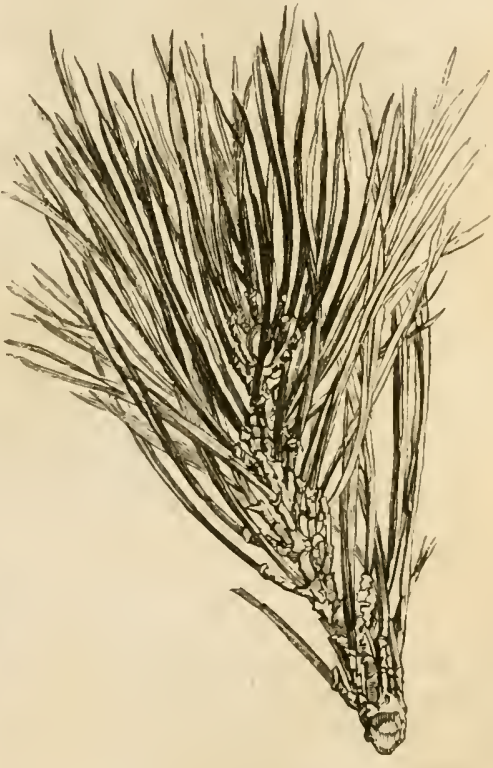

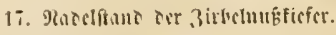

4) Iir Birbelnufficfer ("truc, Bürme, Büridte, Pimss (Cembra) nemet

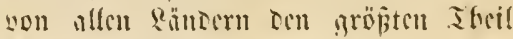

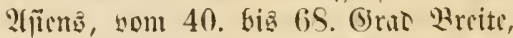

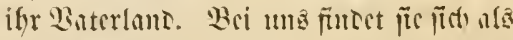
Malcham mur in S!nrol, Defterreid mo

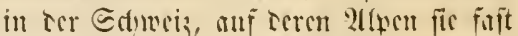

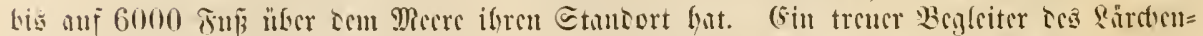

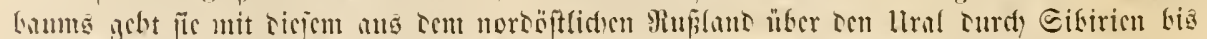

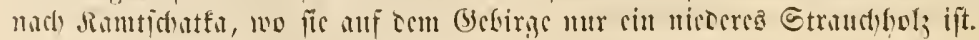

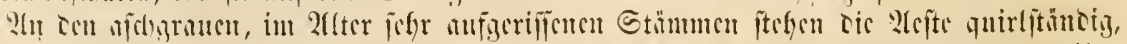

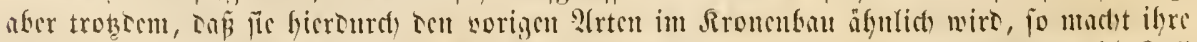

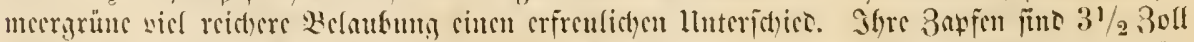

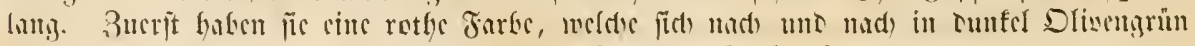

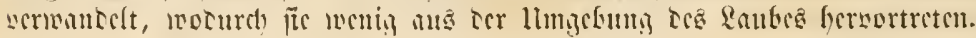





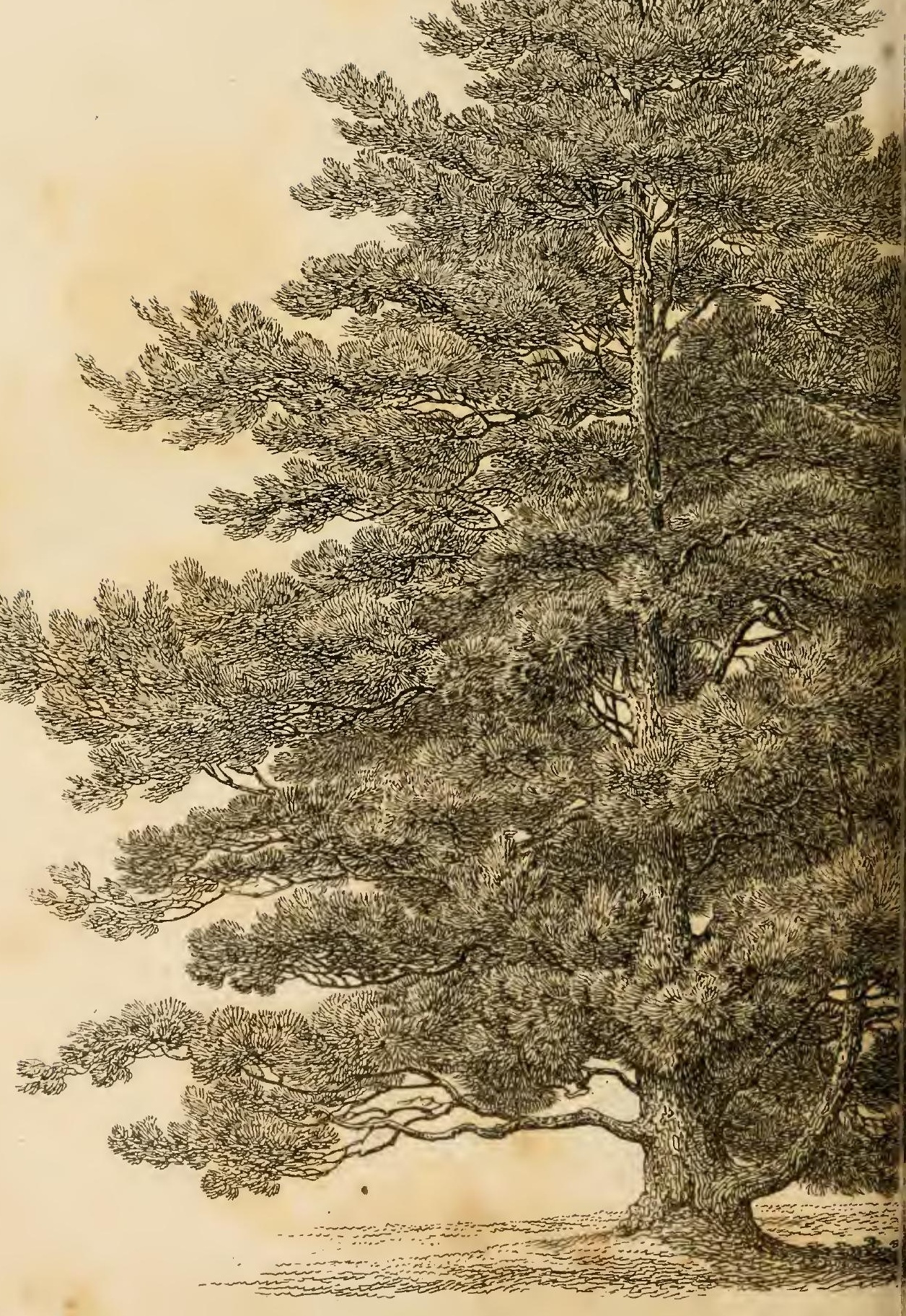
5. 1.

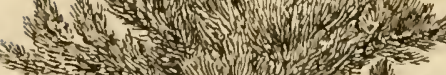

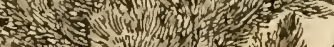

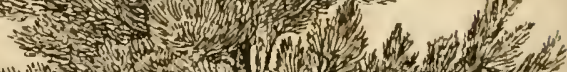

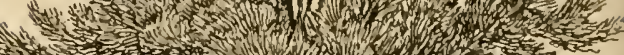
t.

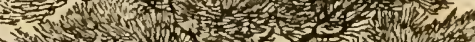
- 151 .

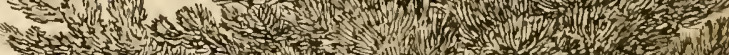

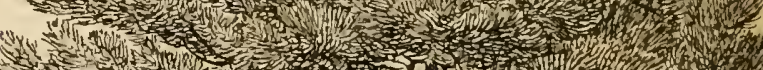

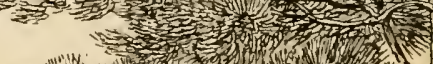

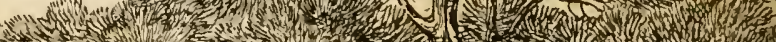

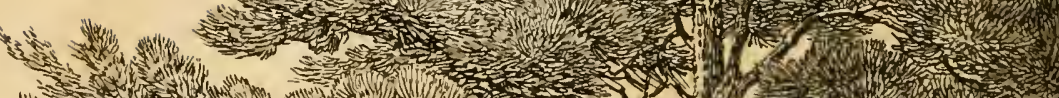

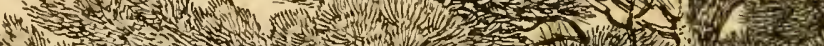

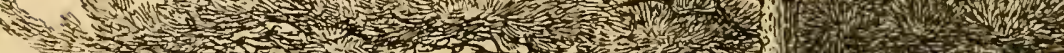
int 7 , s. H. V.1.

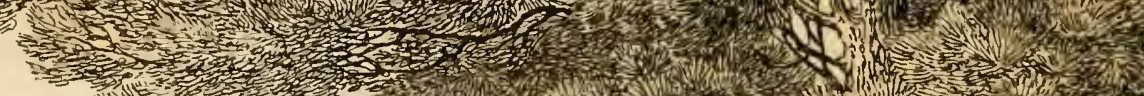

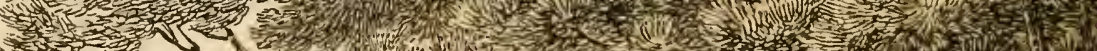

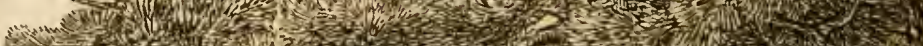

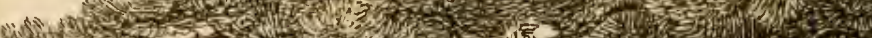
r.

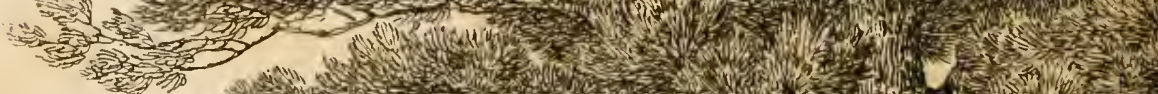





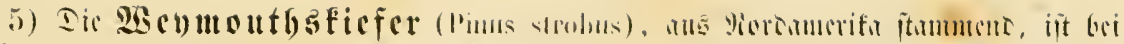

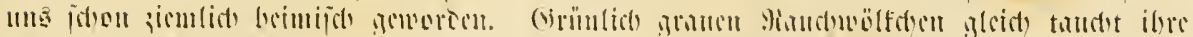

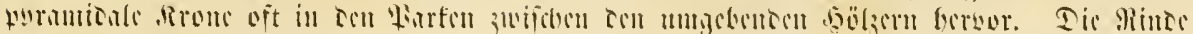

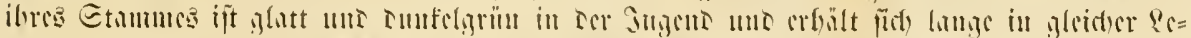

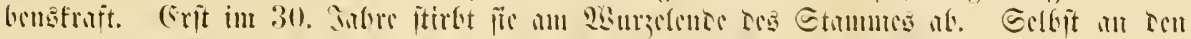

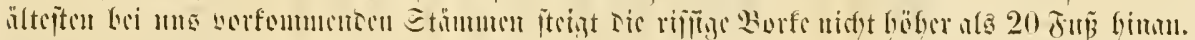

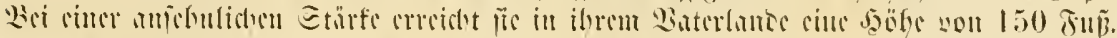

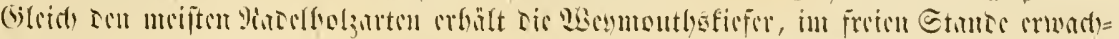

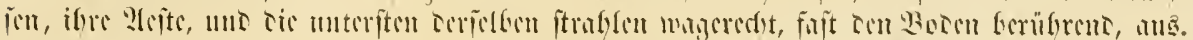

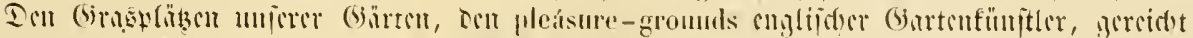

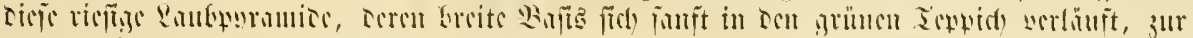

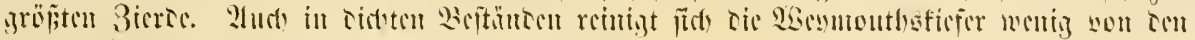
ticferen ?leitent.

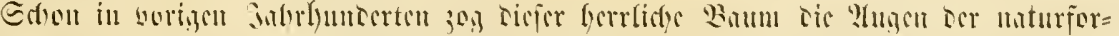

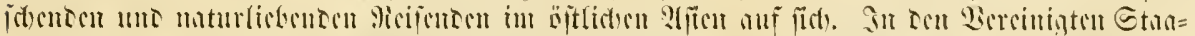

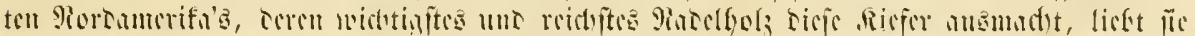

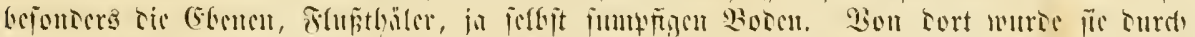

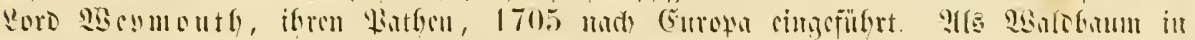

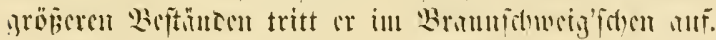

\section{D $\mathfrak{i} \mathfrak{i} \mathbb{E} \mathfrak{i} \mathfrak{i} \mathfrak{i}$}

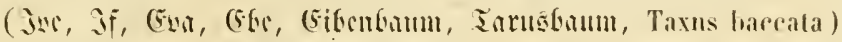

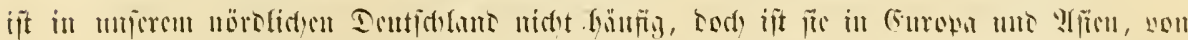

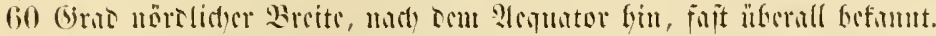

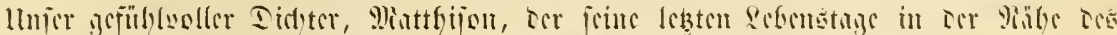

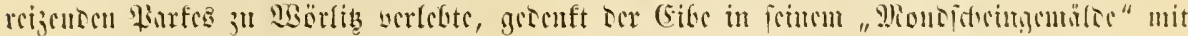
con Bisortell:

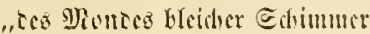

Beitrablt ric sütern (5ibeu

Der Etciten Micicrei."

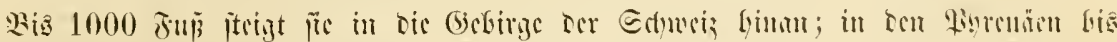

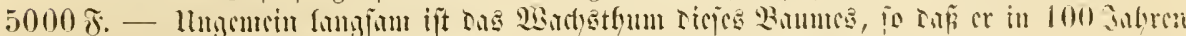

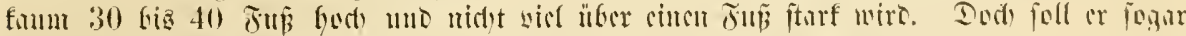

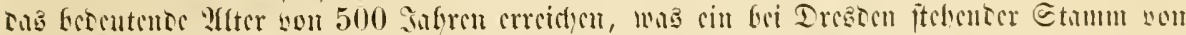

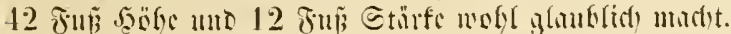

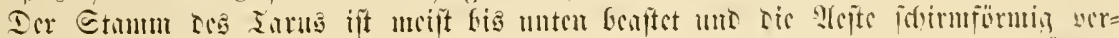

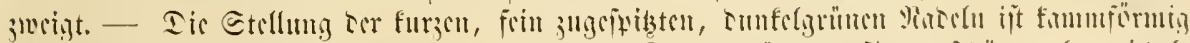

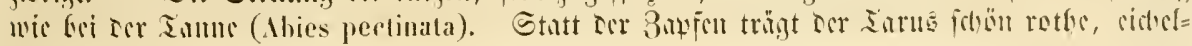
förmilge Becrent.

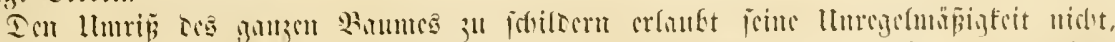

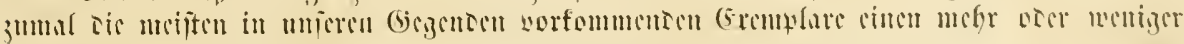

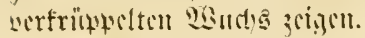

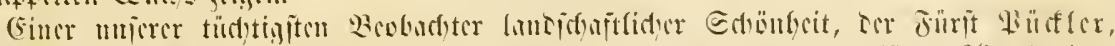

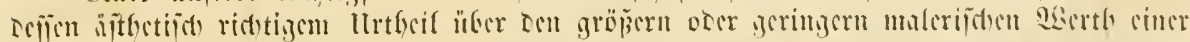

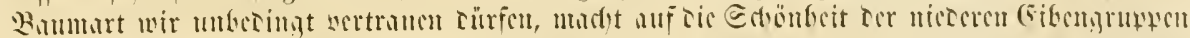

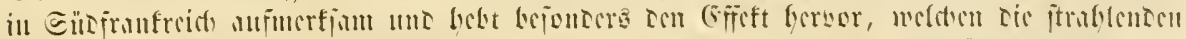

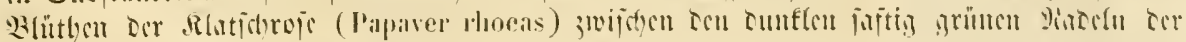

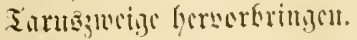




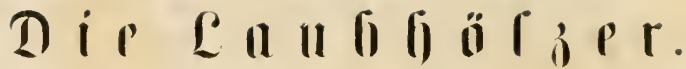

\author{
Dit迁iclj。 \\ Sor 3 anum ift meinte slirdse,

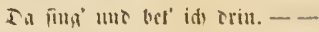 \\ Moricn.
}

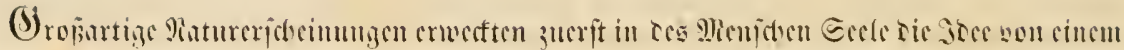

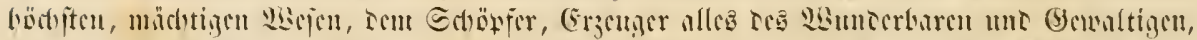

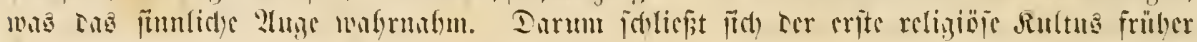

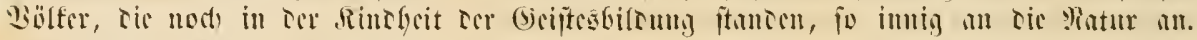

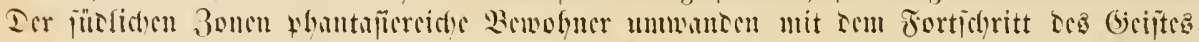

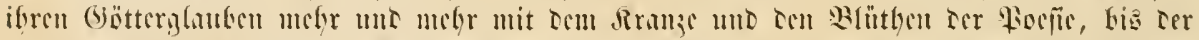

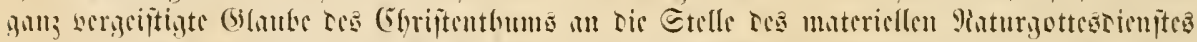

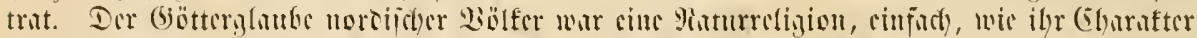

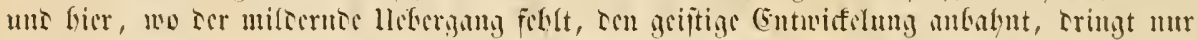

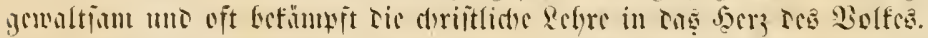

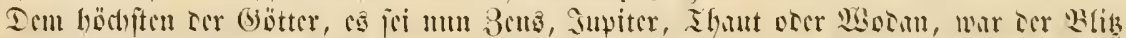

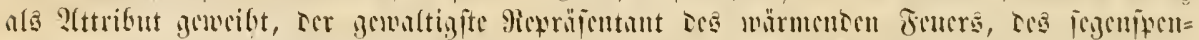

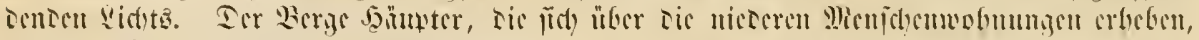

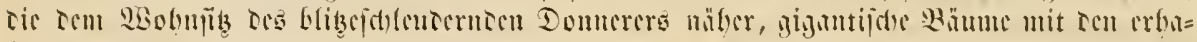

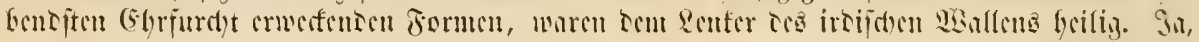

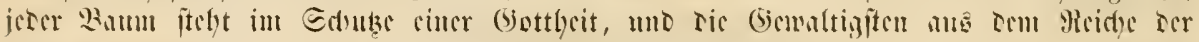

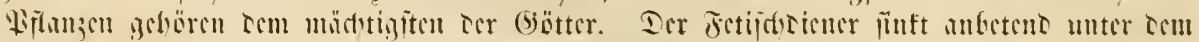

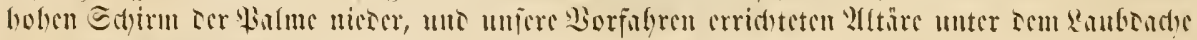
folofinaler Eidubüme.

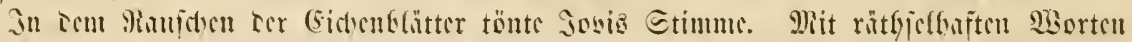

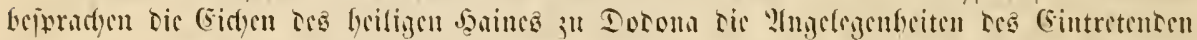

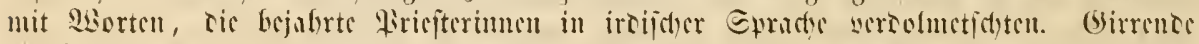

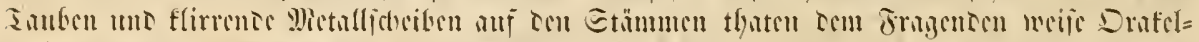

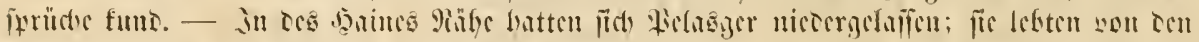

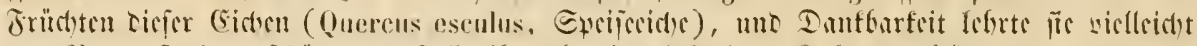

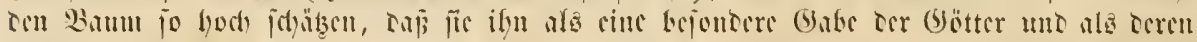
gicbling eforten.

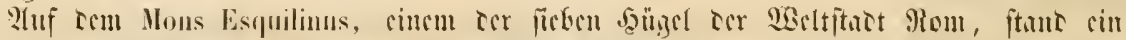

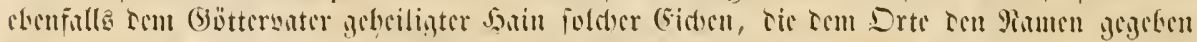

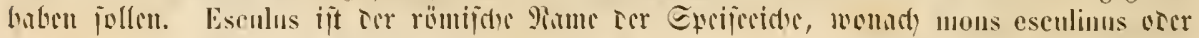

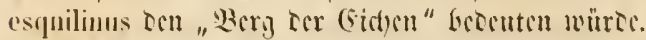

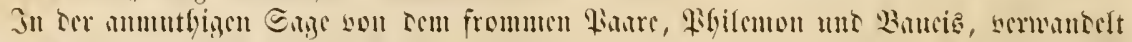

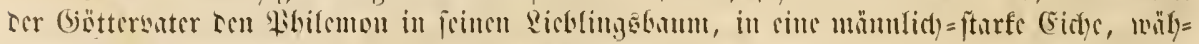

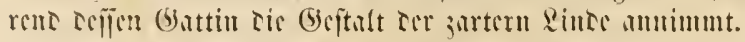

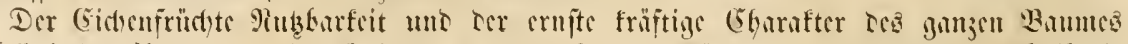

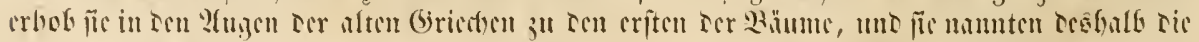

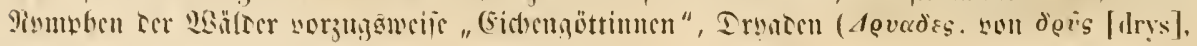




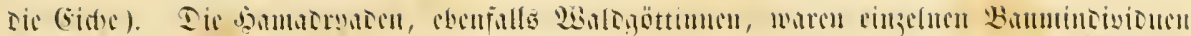

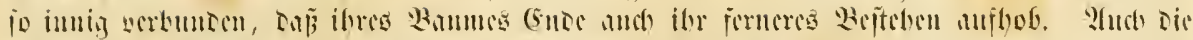

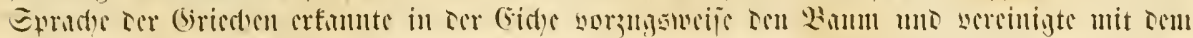

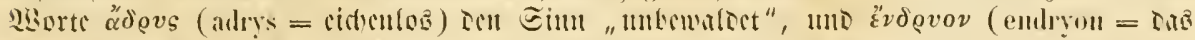

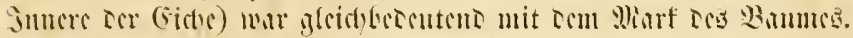

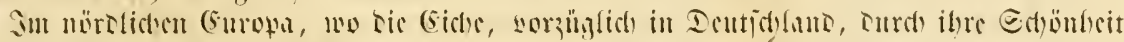

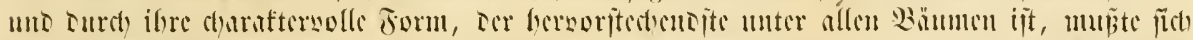

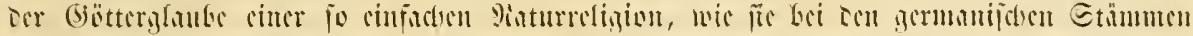

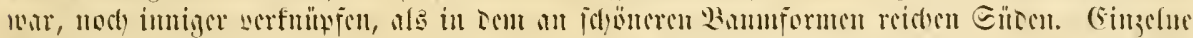

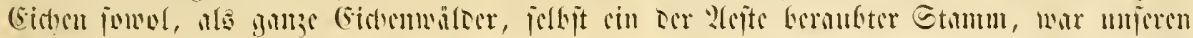

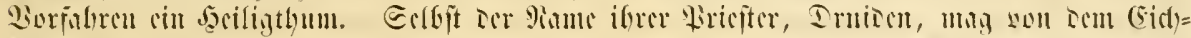

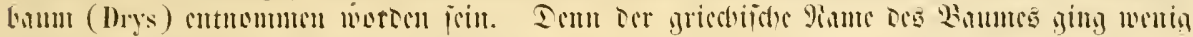

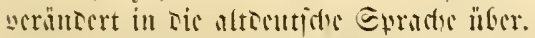

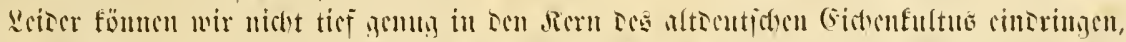

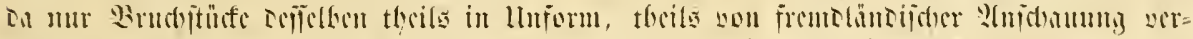

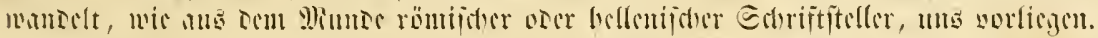

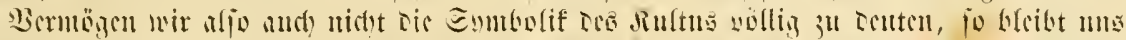
แ2uigitens

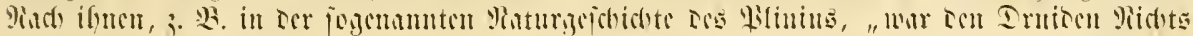

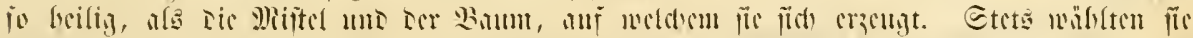

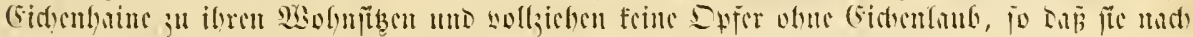

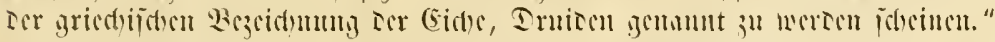

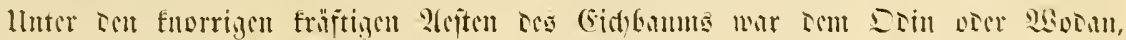

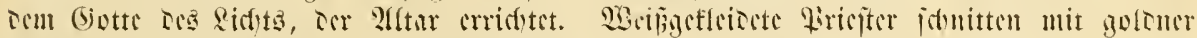

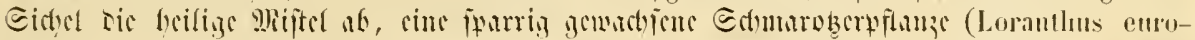

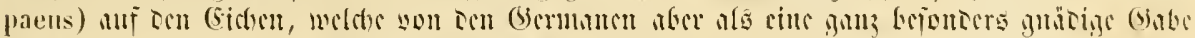

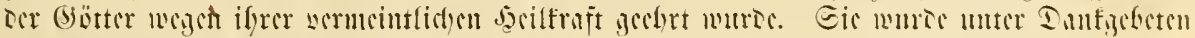

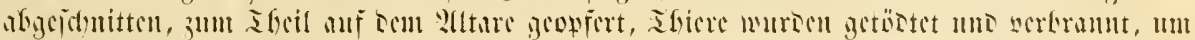

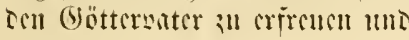

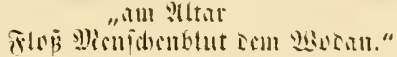

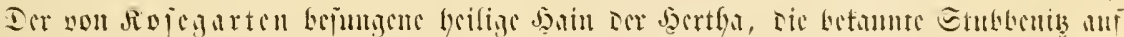

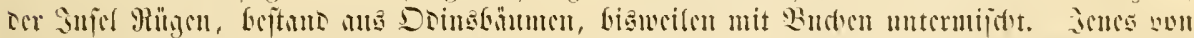

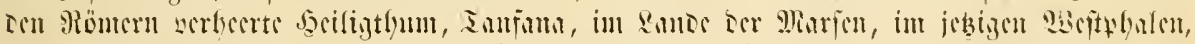

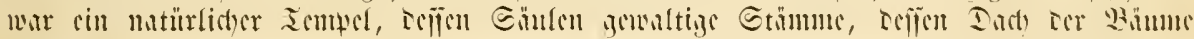
Rauf unt.

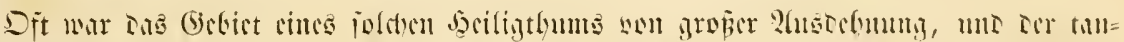

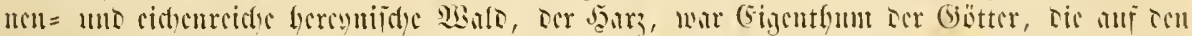

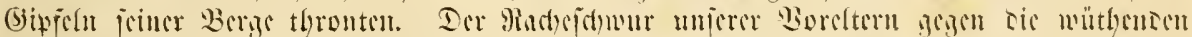

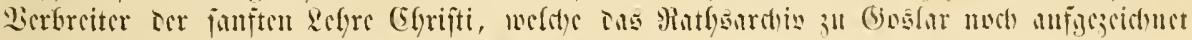

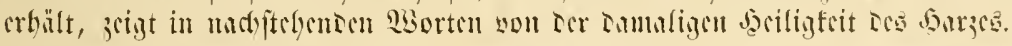

.,Hilli kroti Woudani — - ilp osk of ten aiskenta karlewi. - _ - Ik slakte ti all Faukia up tinen iliken Arlisbeka."

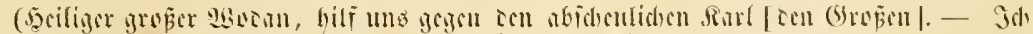

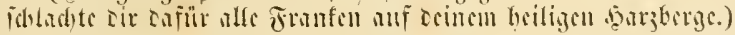

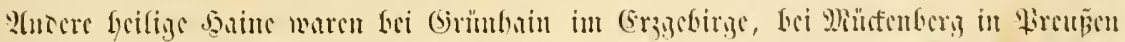

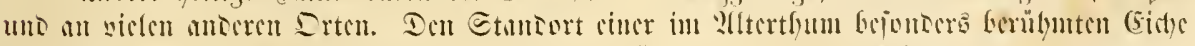

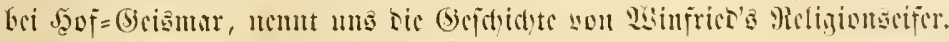

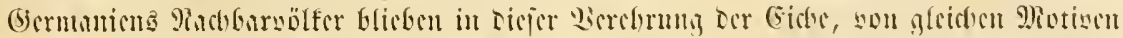

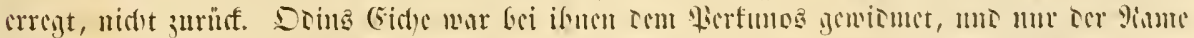

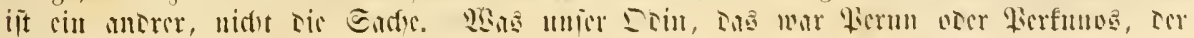




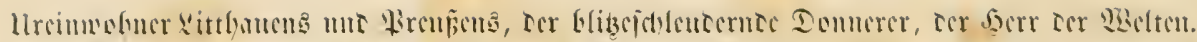

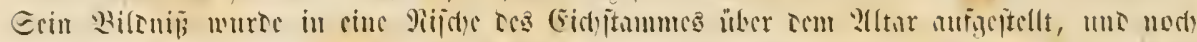

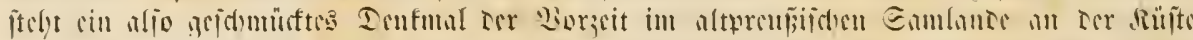

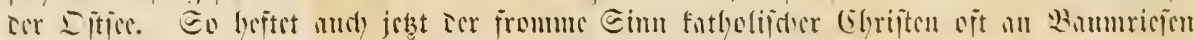

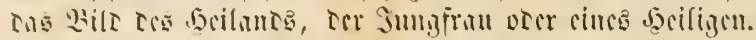

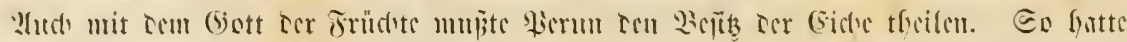

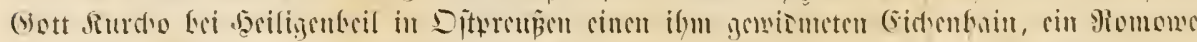

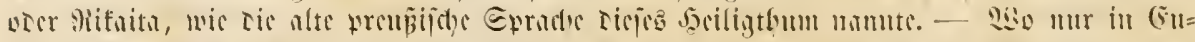

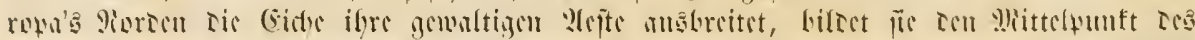

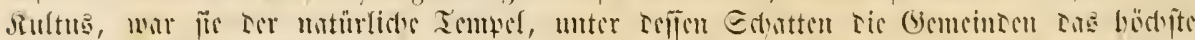

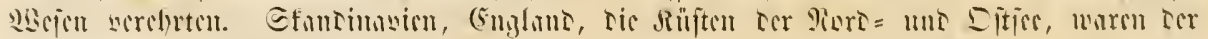

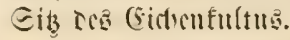

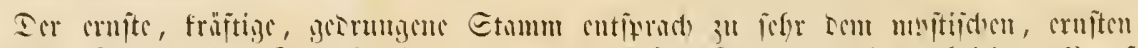

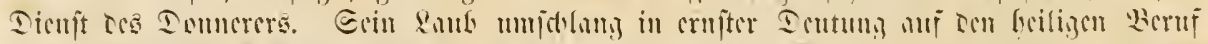

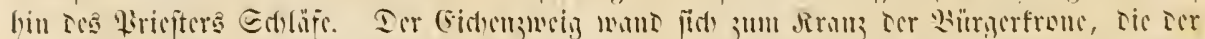

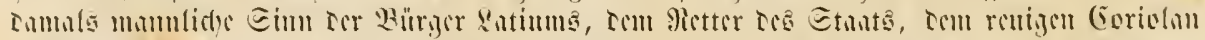

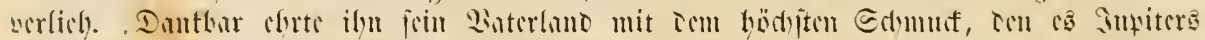

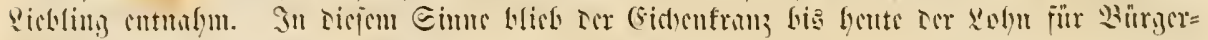
titijents.

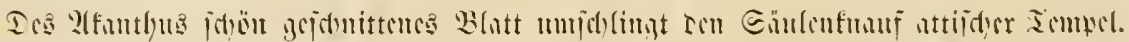

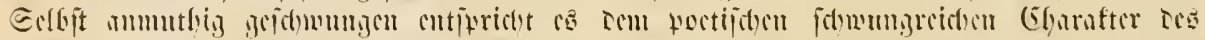

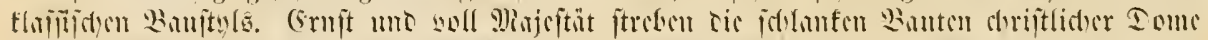

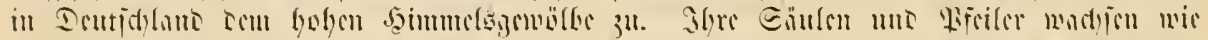

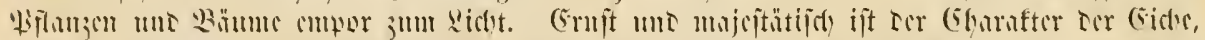

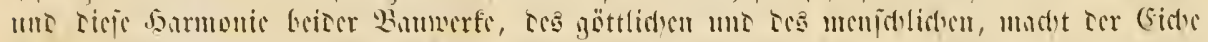

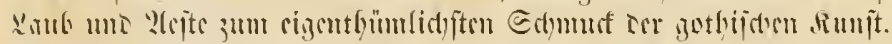

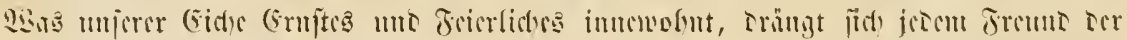

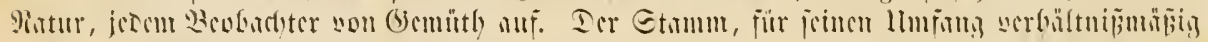

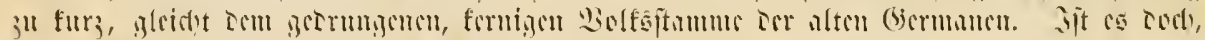

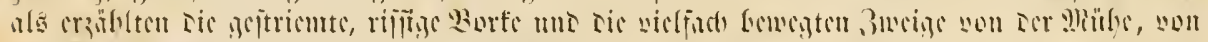

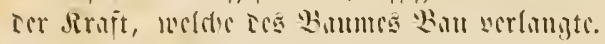

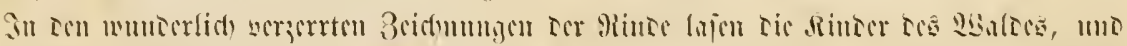

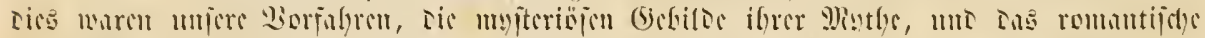

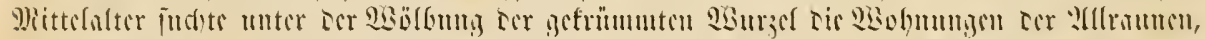
DEsidetel uno E.merre.

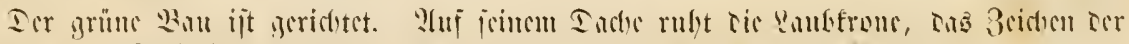

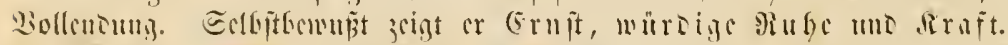

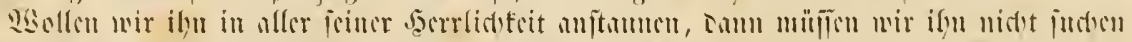

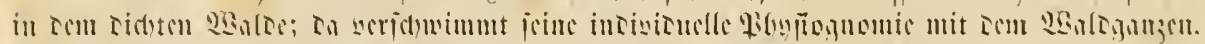

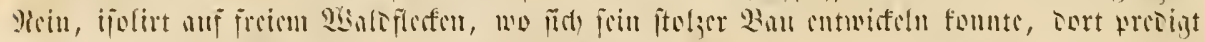

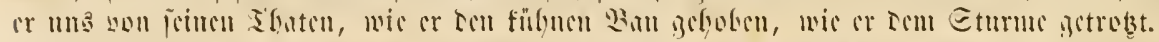

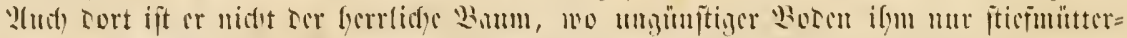

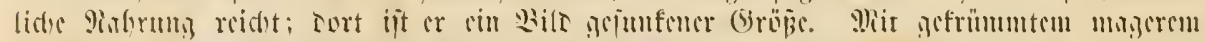

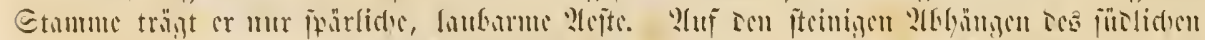

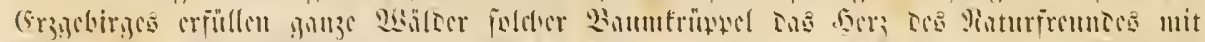

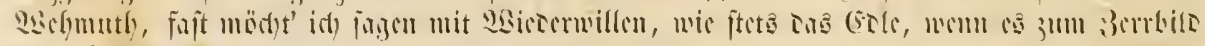
uremintilitit.

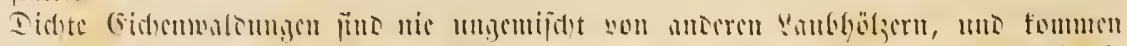

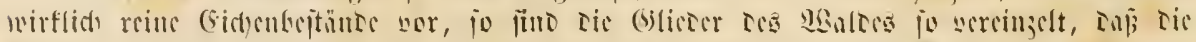

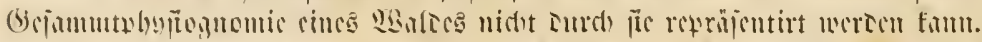

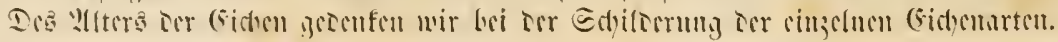




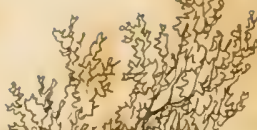

Sitis 3

3.

H.t.



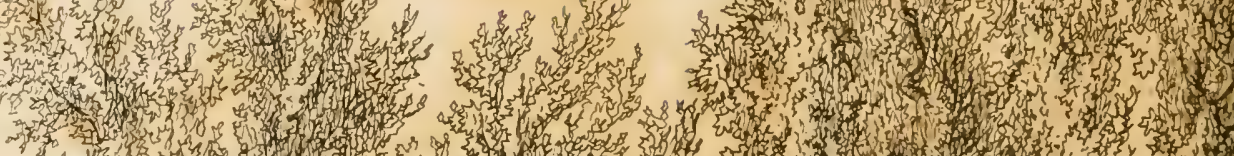
$4 x^{2}$

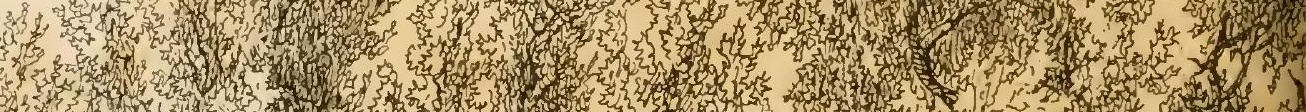

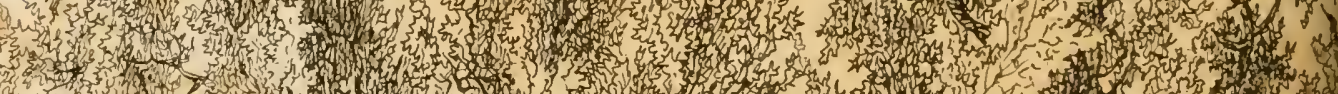
H. 1.7.

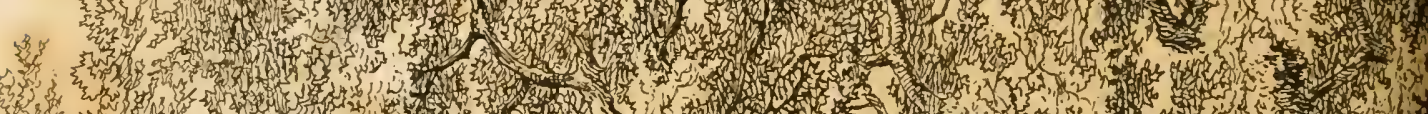
W.

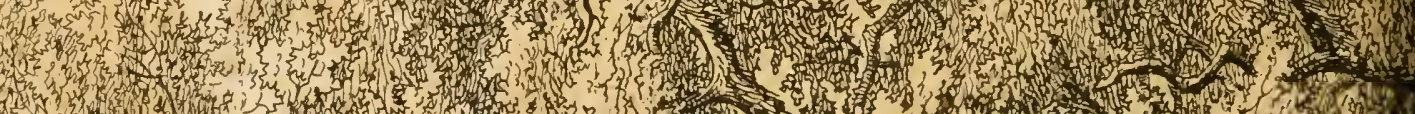

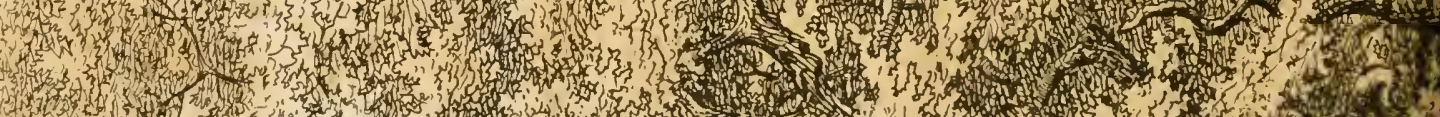

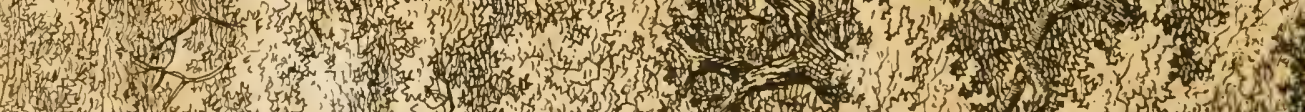
-

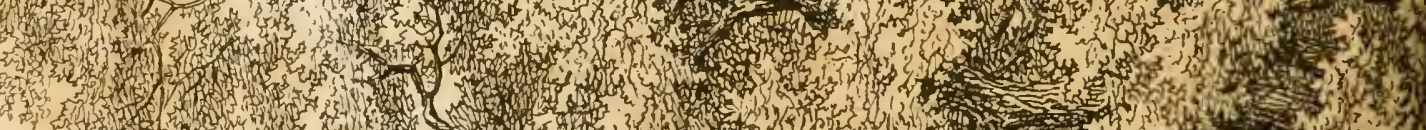

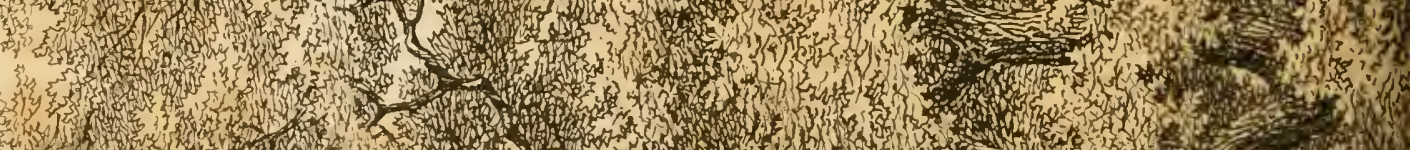
H. r. 3

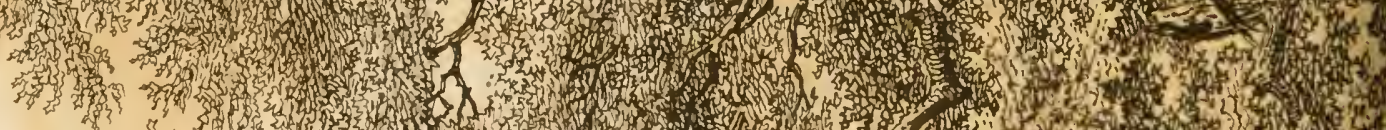

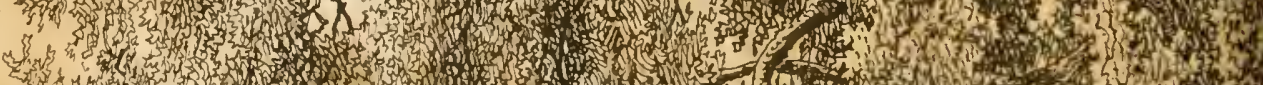
2.7. H. 34. 2. 2.

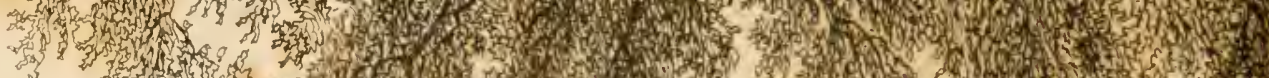

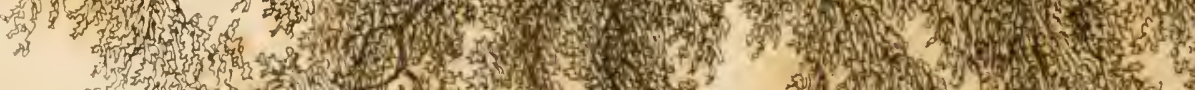
H.

$$
\begin{aligned}
& \text {-3) } \\
& \text { द म }
\end{aligned}
$$

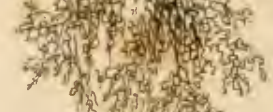

$$
\begin{aligned}
& \text { son enth }
\end{aligned}
$$


s

(1)

aris

Esent

sulo

1.

30 \&

Ling in

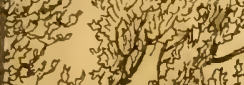

5.

5.

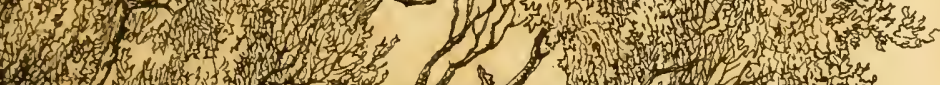

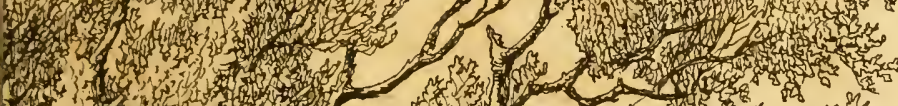
14) (1)

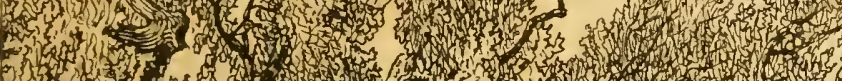

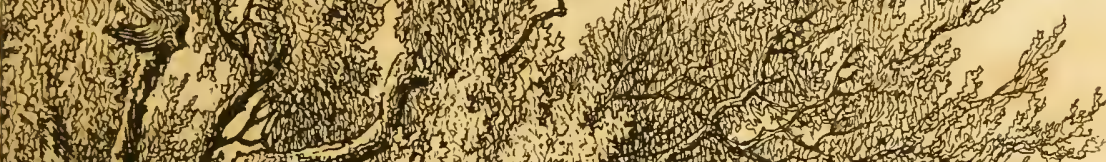
1.

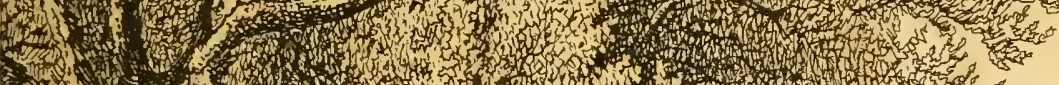

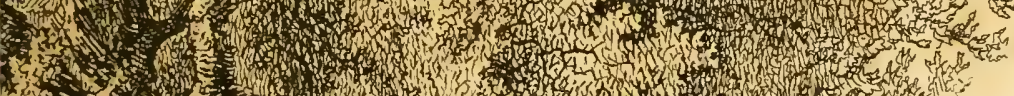

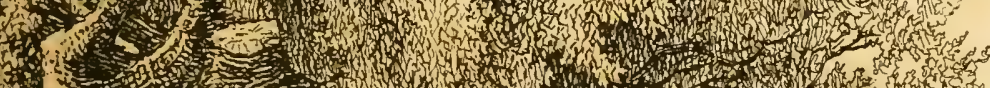
And द

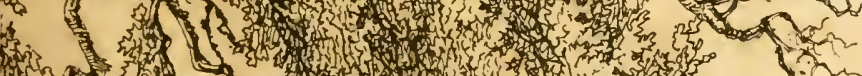
(n) दुर

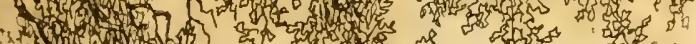

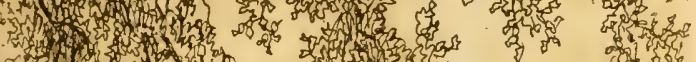

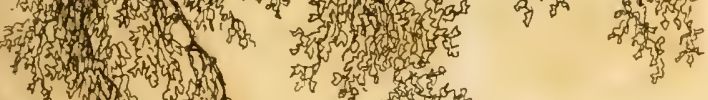
年

$$
\text { My }
$$

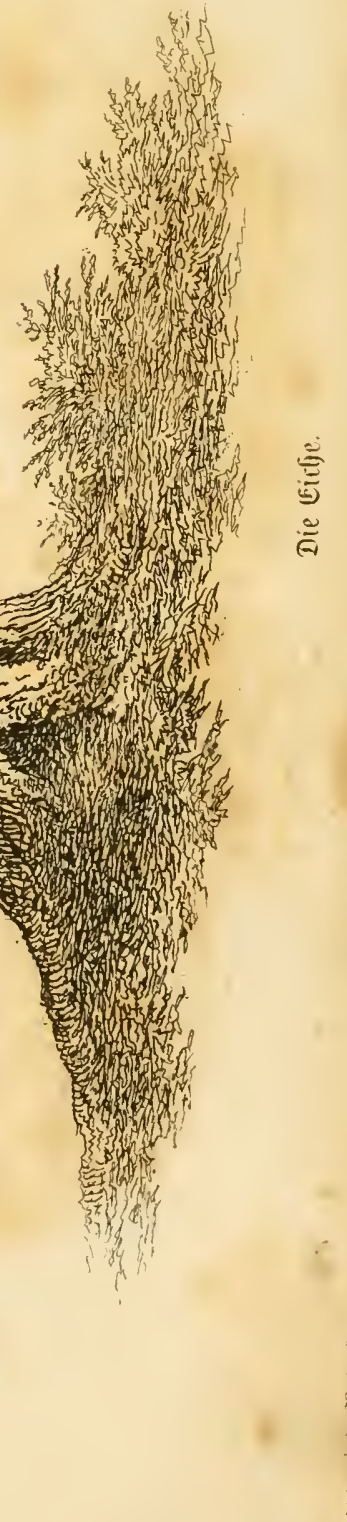





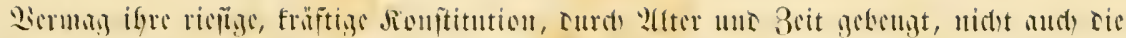

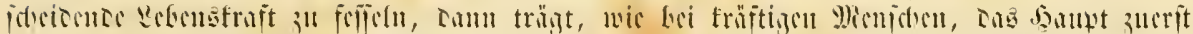

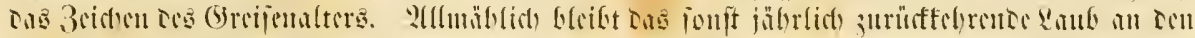

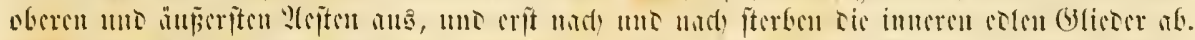

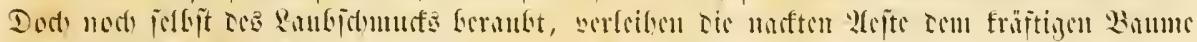
cincu fittoresten (sfict.

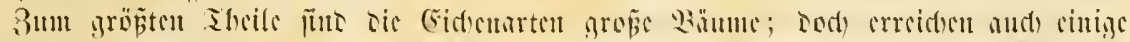

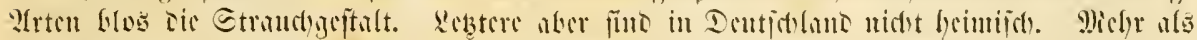

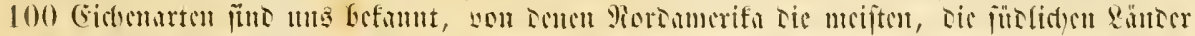

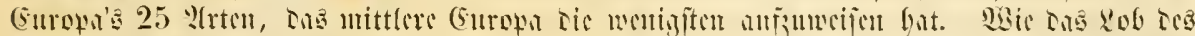

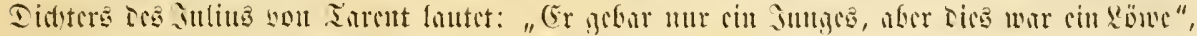

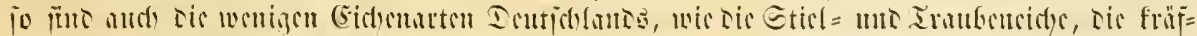

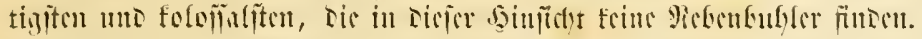

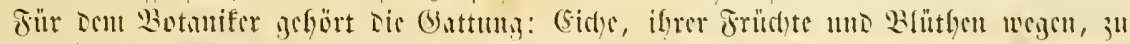

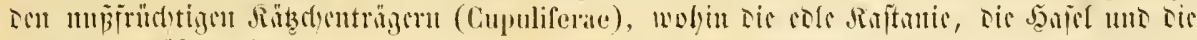

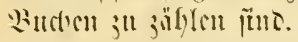

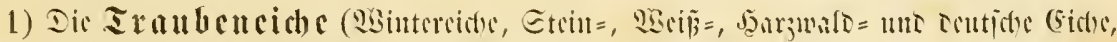

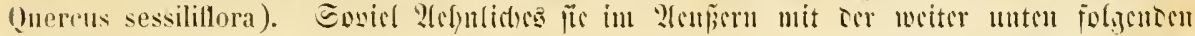

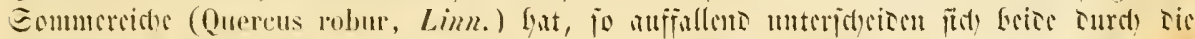

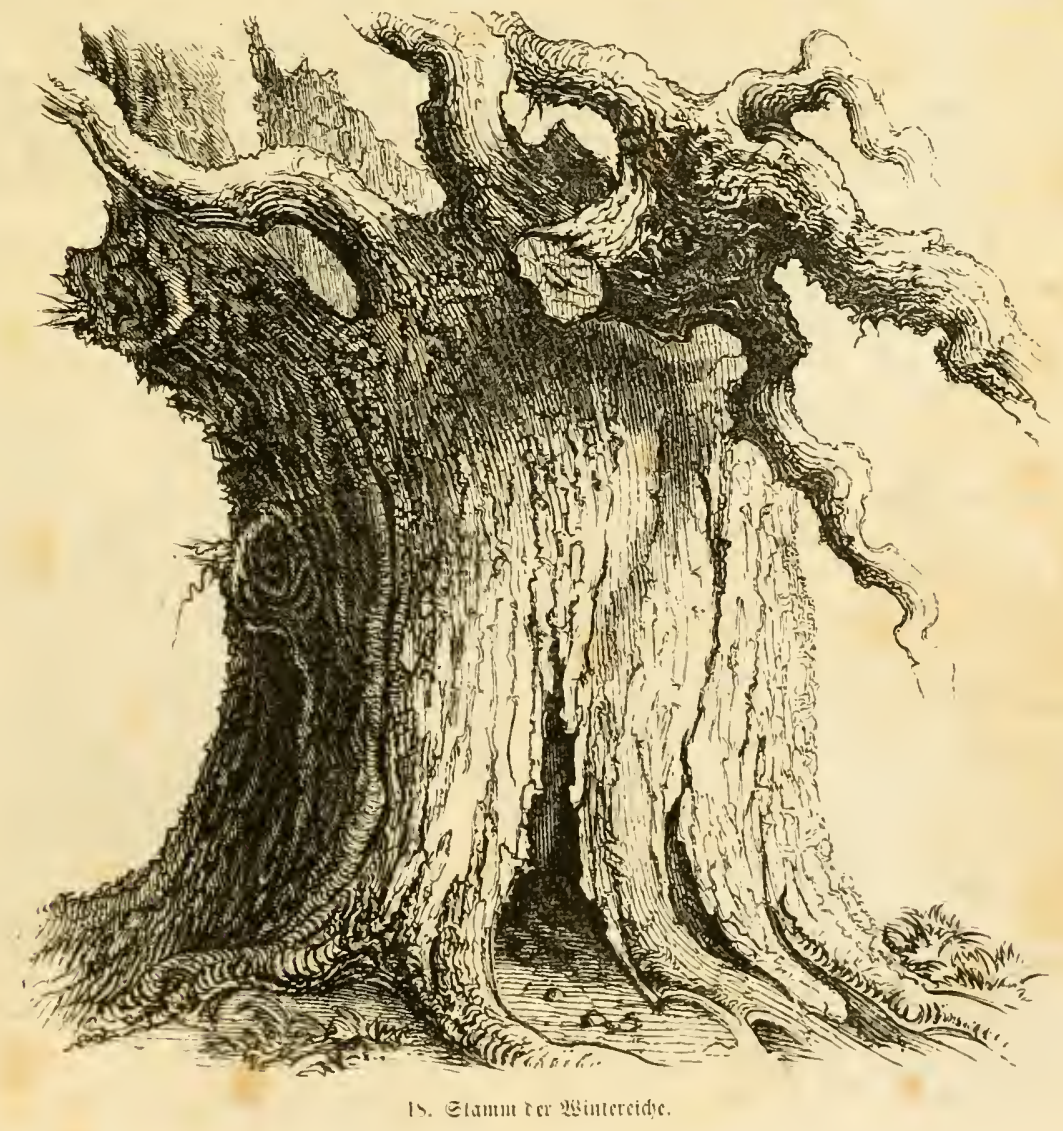




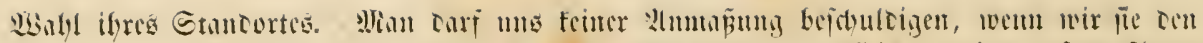

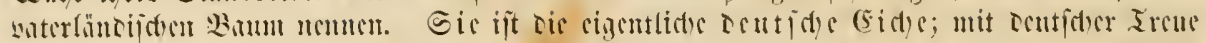

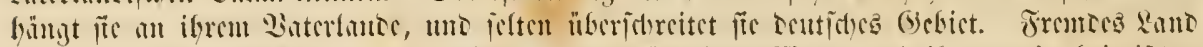

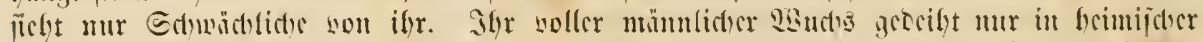

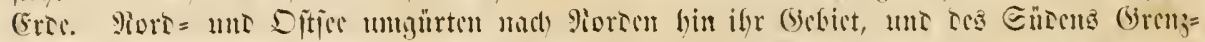

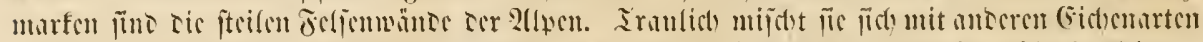

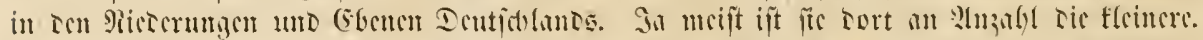

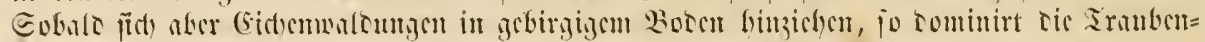

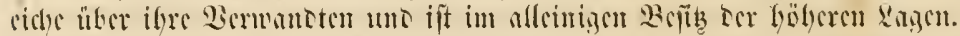

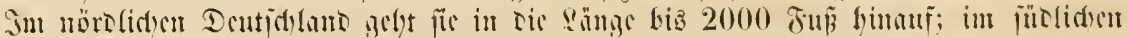

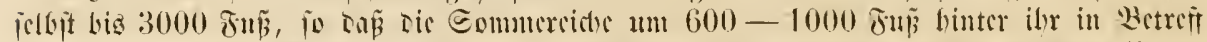

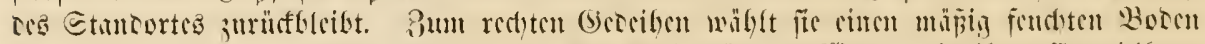

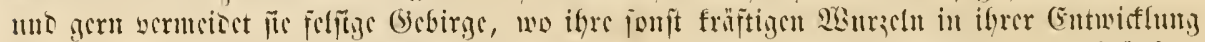

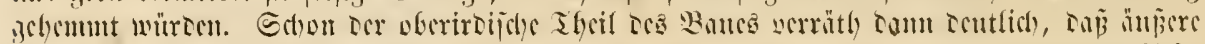
(Sinflinjic jein Bast) jurfictblibon, ift and) Sor Jironenban türfitiger.

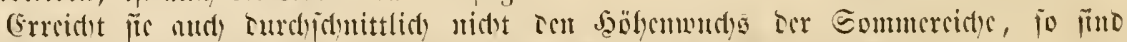

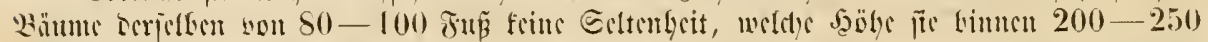
sidoren craticts.

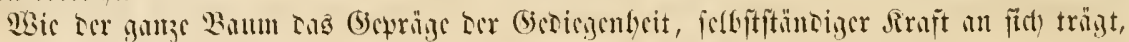

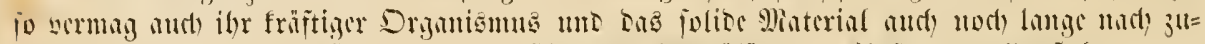

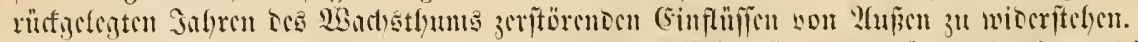

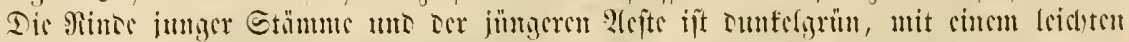

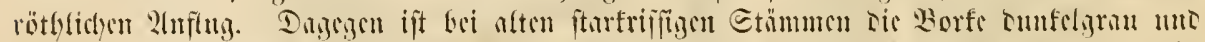

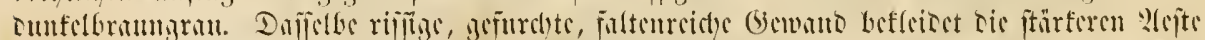

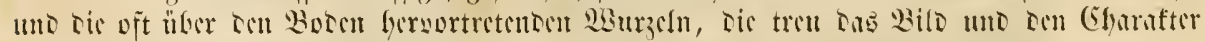

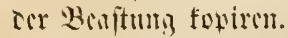

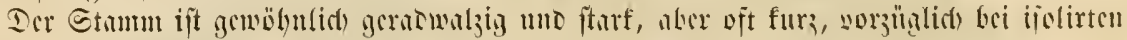

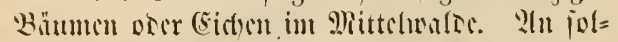

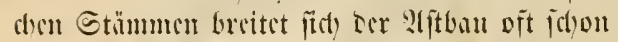

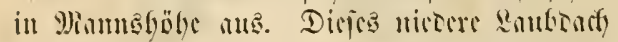

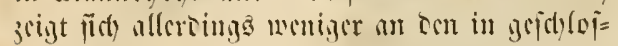

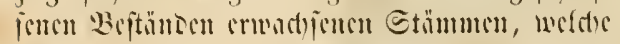

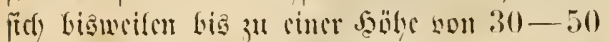

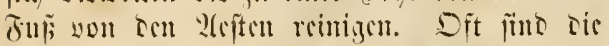

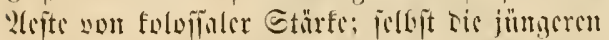

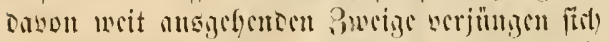

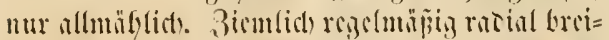

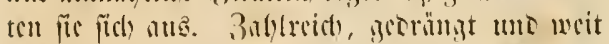

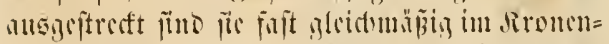

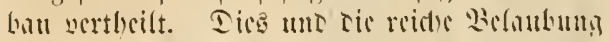

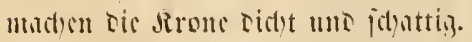

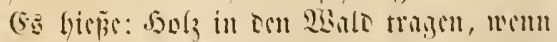

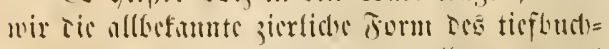

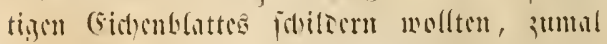

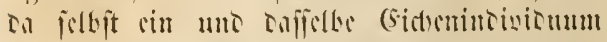

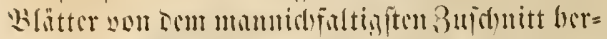

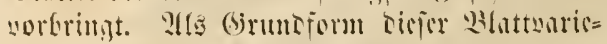

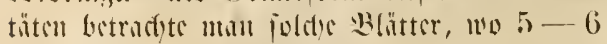

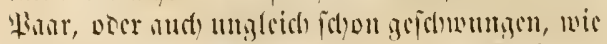

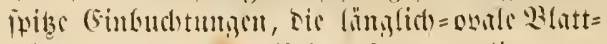

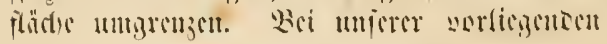

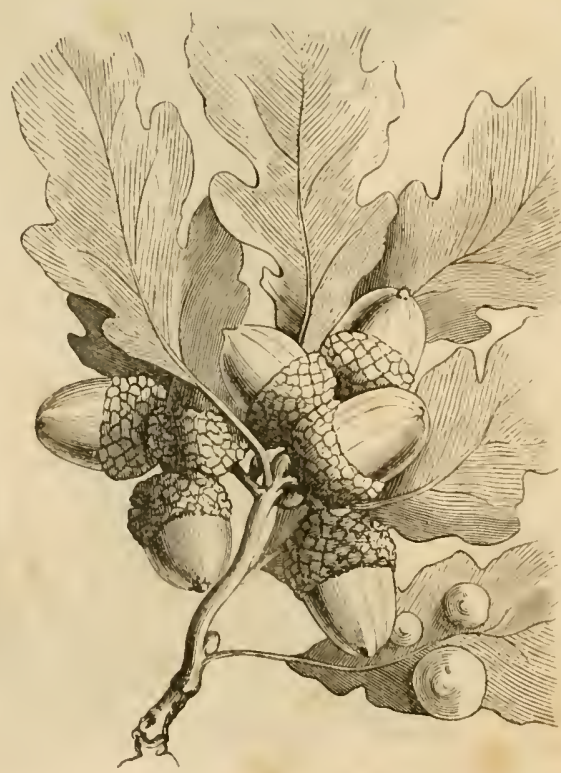

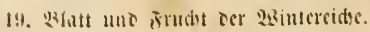


(Fit)

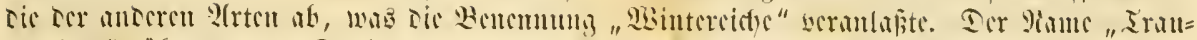

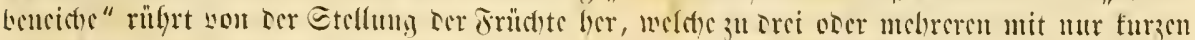

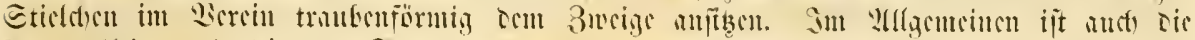
Frudt fleinter als bic oor Eommercide.

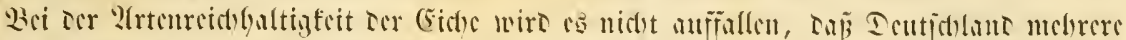

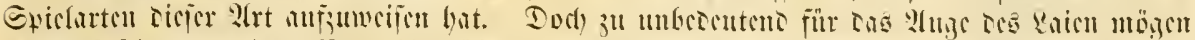

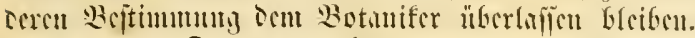

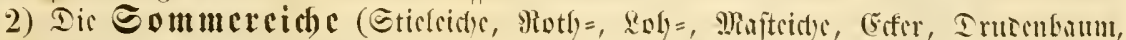

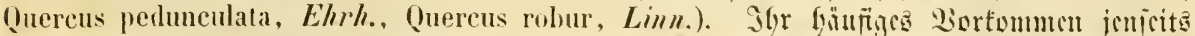

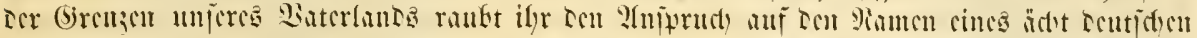

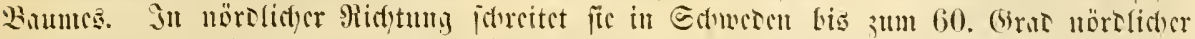

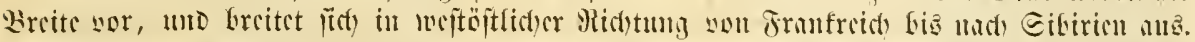

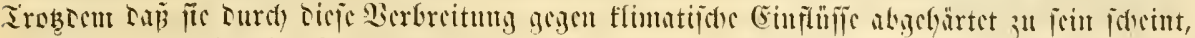

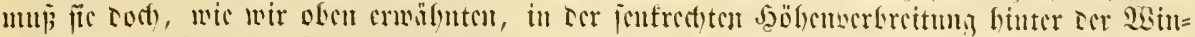

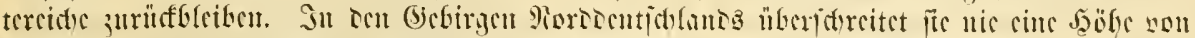

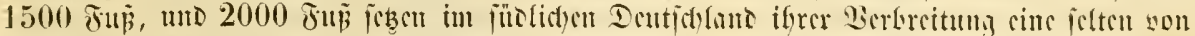

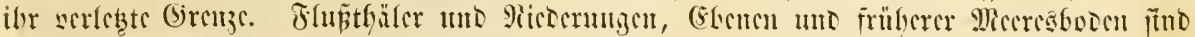

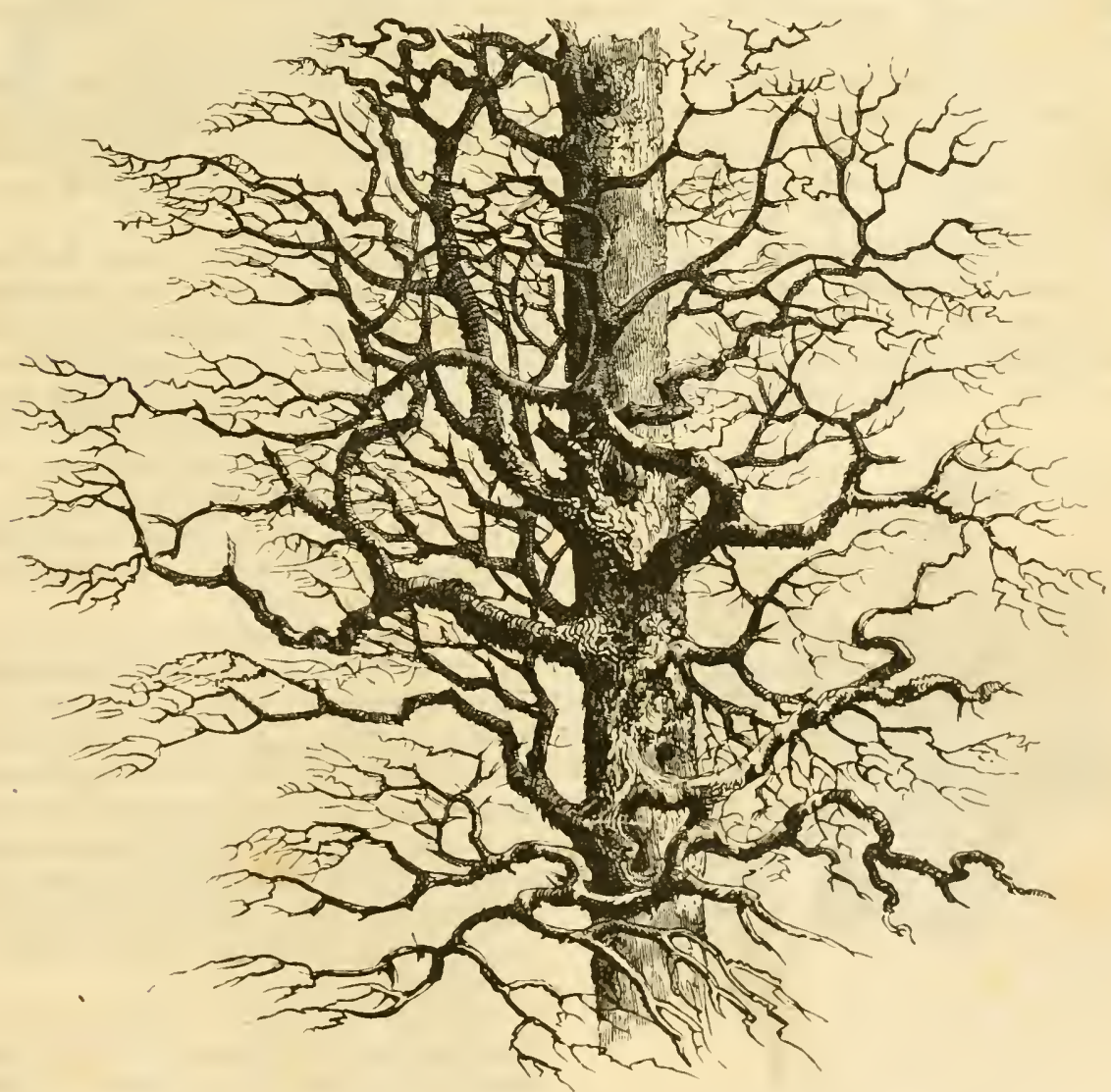

20. Whittelitutut rer $\Xi$ ommercithe. 


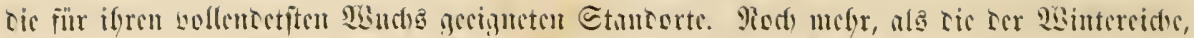

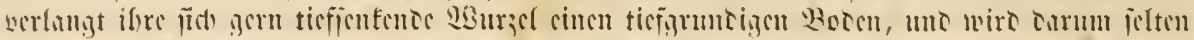

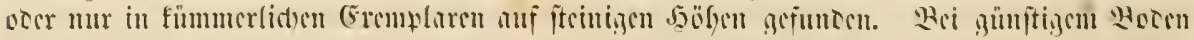

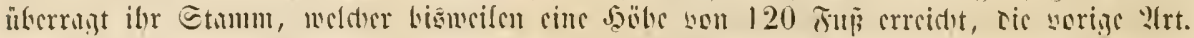

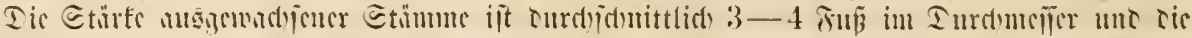

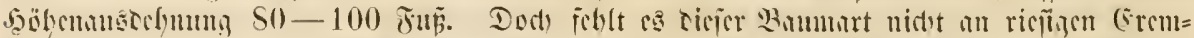

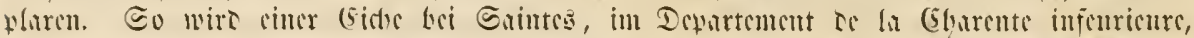

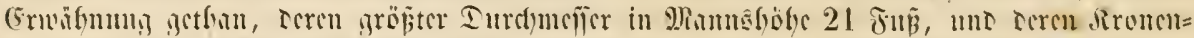

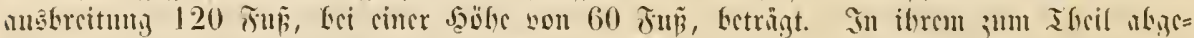

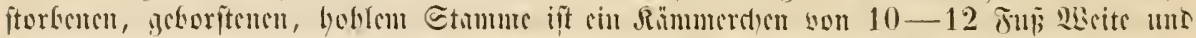

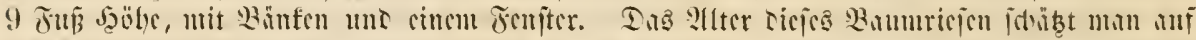
$1800-2000$ Sulire.

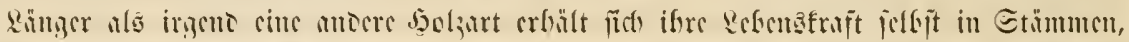

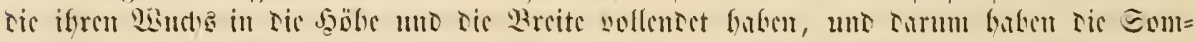

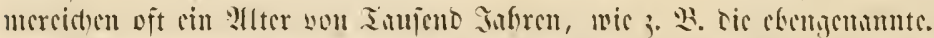

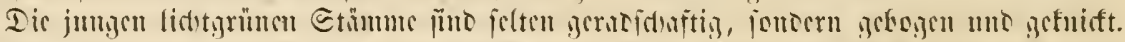

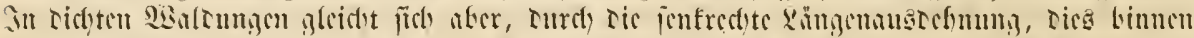

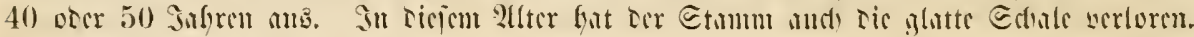

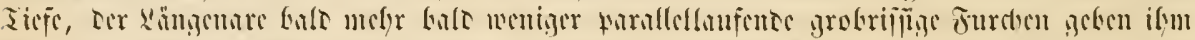

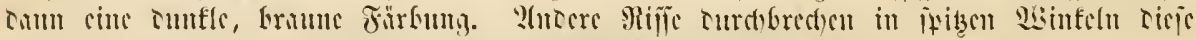

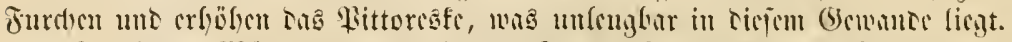

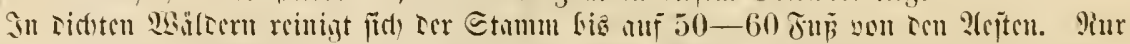

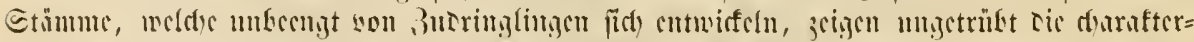

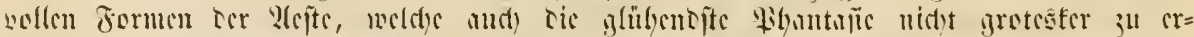

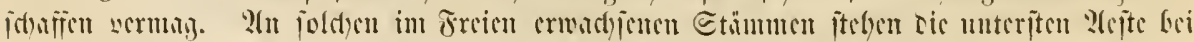
$21)-30$ गші.

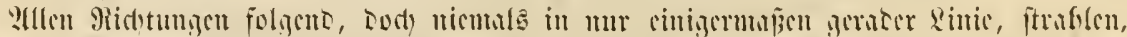

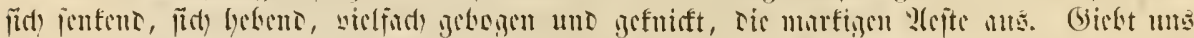

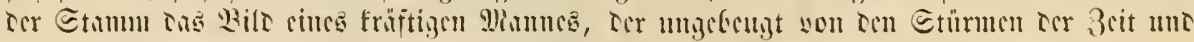

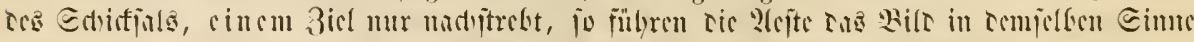

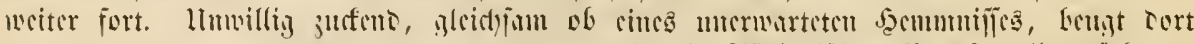

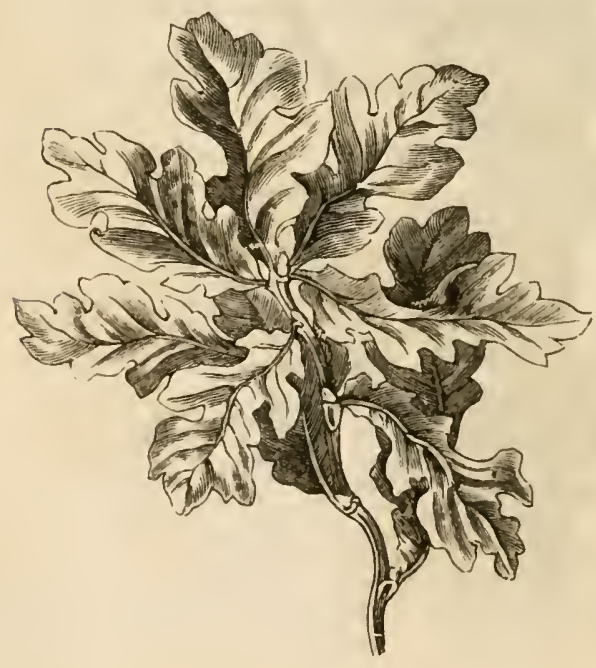

21. H/attitune cer Esmmereidis.

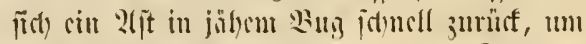

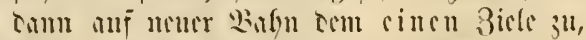

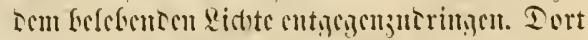

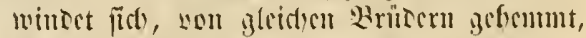

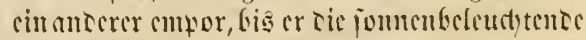
(Srenze Ior Jrons erreistt.

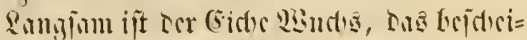

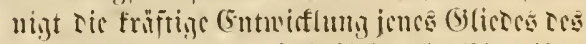

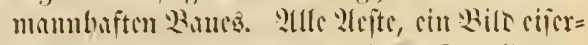
ner Bisharrlichfeit, getwart mit Rinft, wollenten

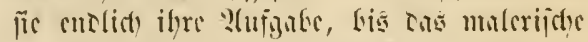

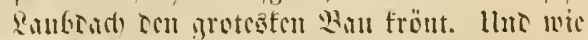

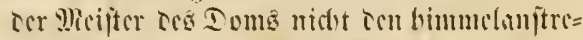

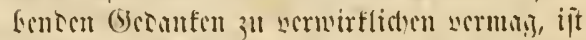

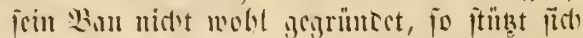

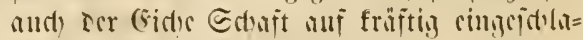
neme sisurzoln.

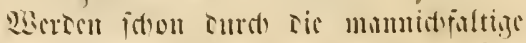
2y

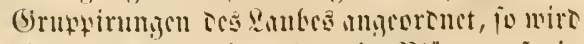

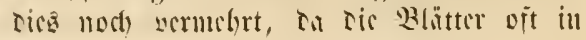




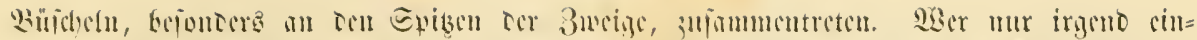

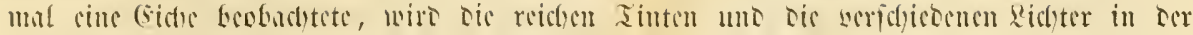

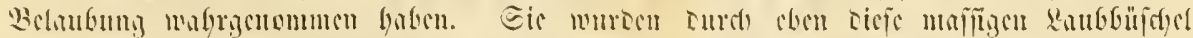

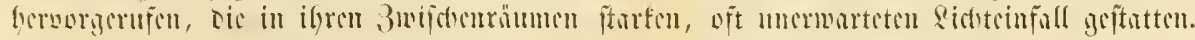

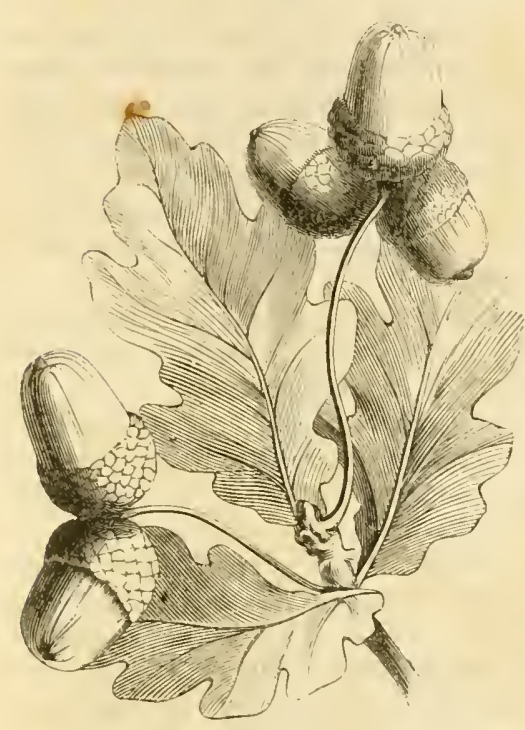

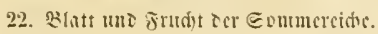

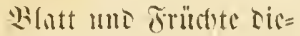
for ?!rt unteriducisen fist) you Ior worigen Durd bis Eäme ter Eticle. Iic Ier Whäter jün fürzer mo tic

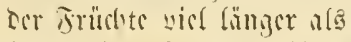

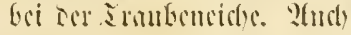
itefin bier nicht mebrere Früdte jo sidft teifommen, fonseru meift cinzelu.

Dion Ion nicfleidt) 20 Evicharter Ior Goummer= ride gecenfen wir me cini= jer fibe bersorftedenten: ?tr ber Sinramiosucicte

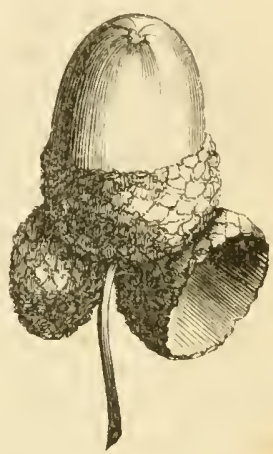

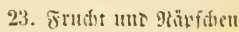
Der Eommereidic.

(Querrus l'astigiata) find

Dic 3wcige ocm Etamme ctwas angetrüst, un=

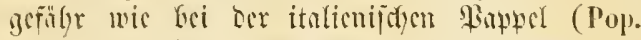

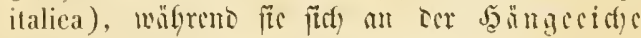

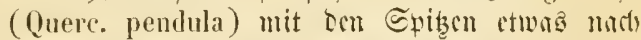
unten meigen. Dis gefoctete (sidfe (Buere. foliis variegatis) hat weipgefleftę, cic 2 int

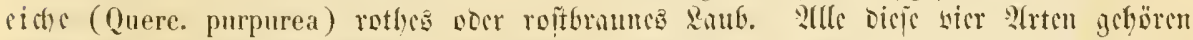

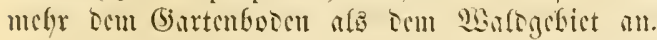

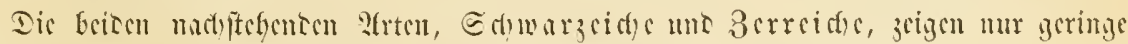

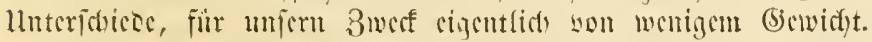

3) Dic Schwarzeiche oter weith harige (Eiche (Quercus pubescens) ijt

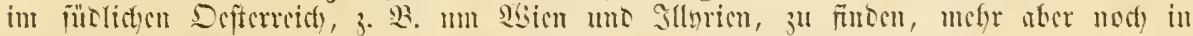

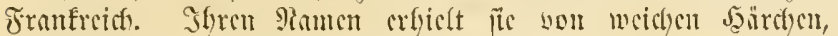
womit Bräter mis Fruct)toctyor bejegt junt.

4) Dis Berretche (öterreidiffye Gicte, Quereus Cerris), in lingarn, Stalicu, Franfreict), Rïrntfocn, Rrain und

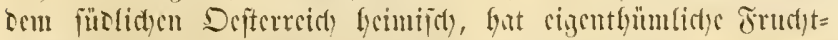

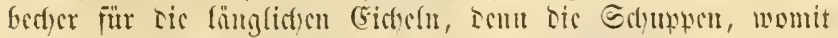

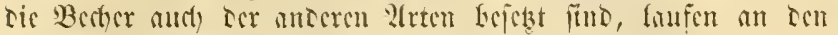

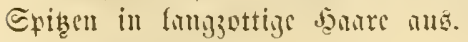

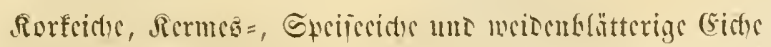

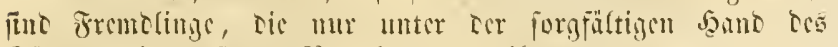
bärtuers in unferem Baterlames getergen.

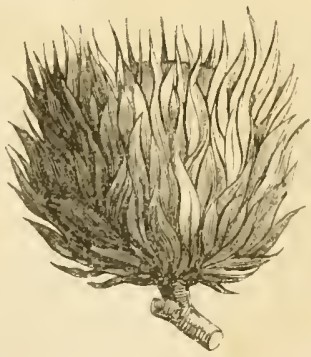

24. iradil ser Berrecidi. 


\section{两ic}

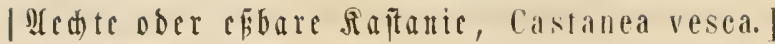

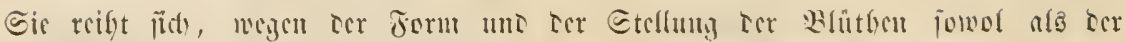

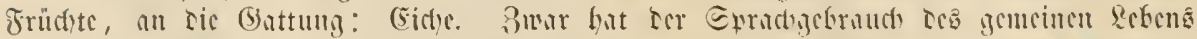

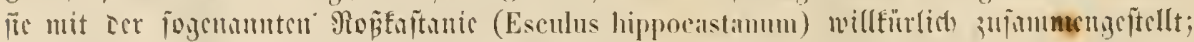

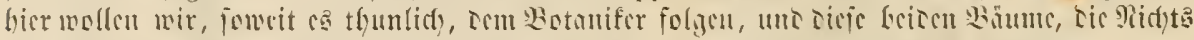

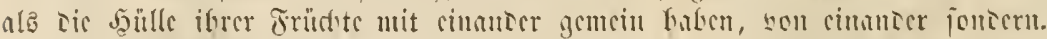

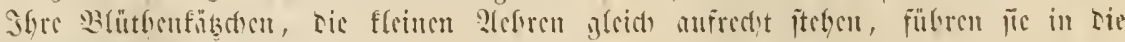

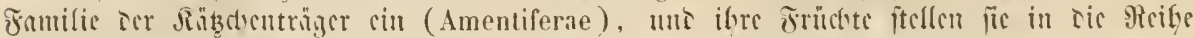

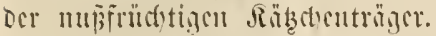

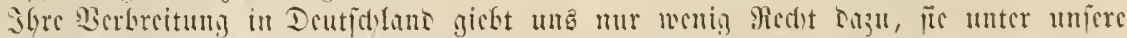

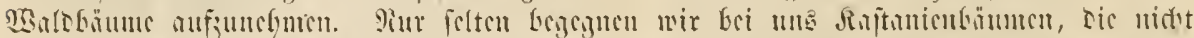

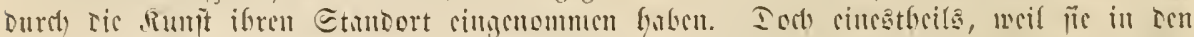

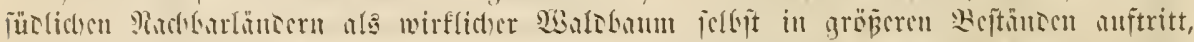

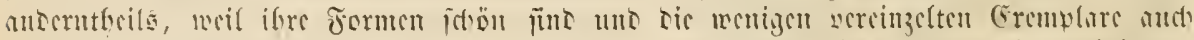

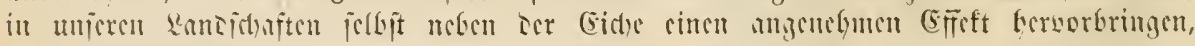
cürfen wir j̈e nidte ilserigelyen.

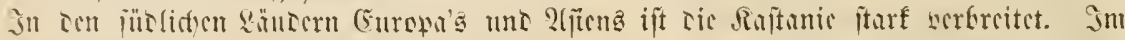

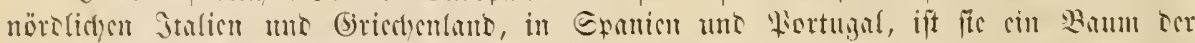

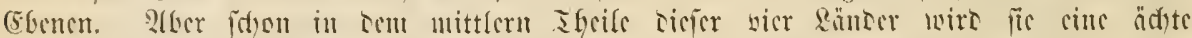

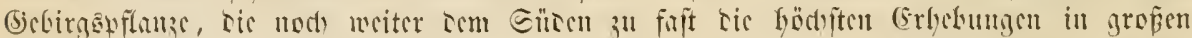

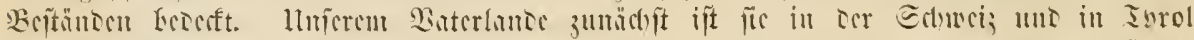

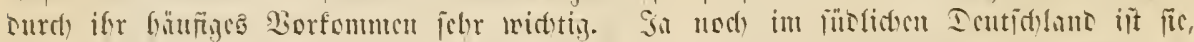

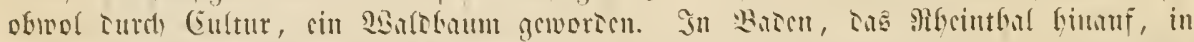

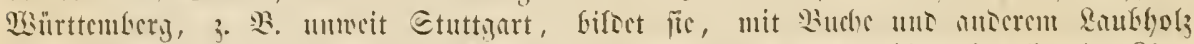

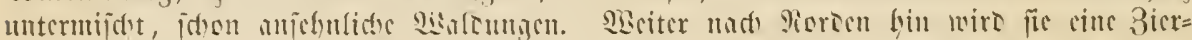

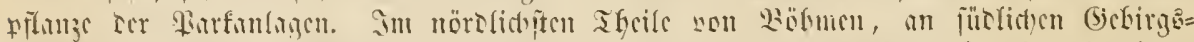

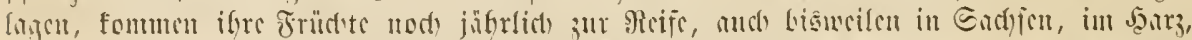

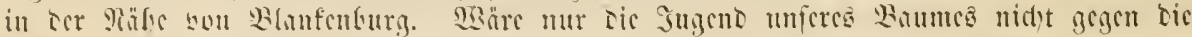

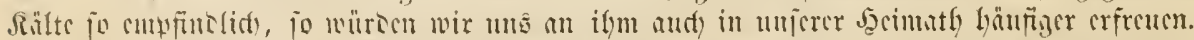

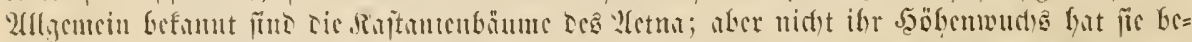

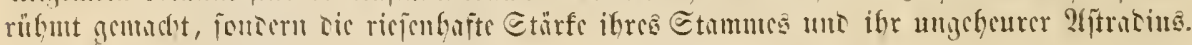

Sil 60 - So sirluren bollen=

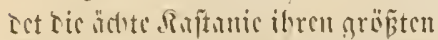

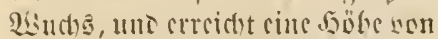

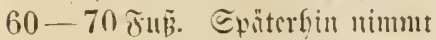

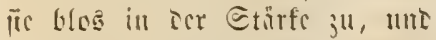

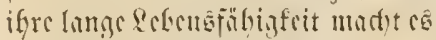

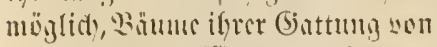

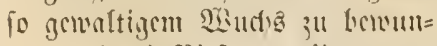

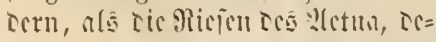

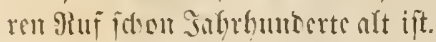

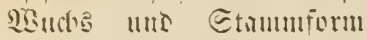
ift Ior Eides fofyr sibulids. Iis Rinte alter Bäume ift fowns? แกD fât chen fo ftarf gerififen, alอ

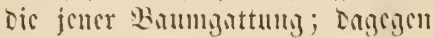

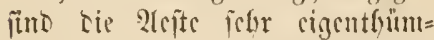

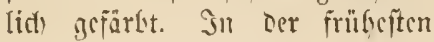

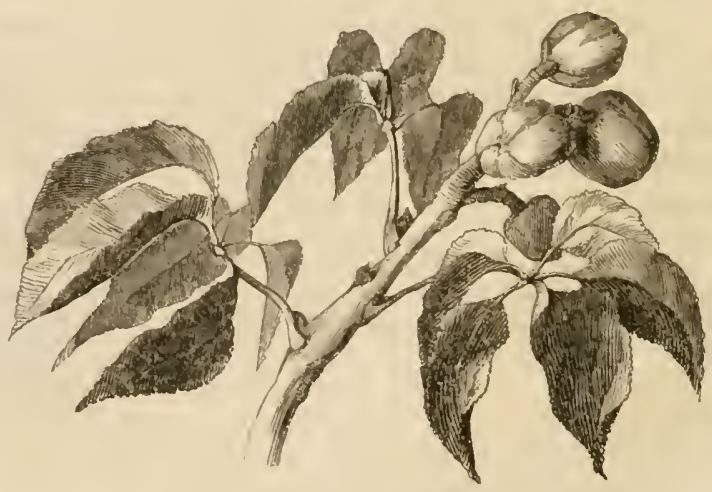

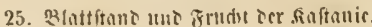




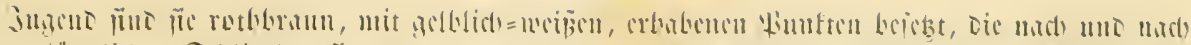

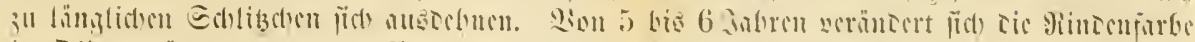

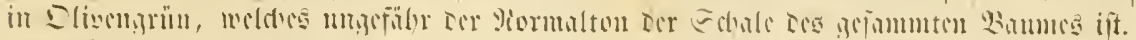

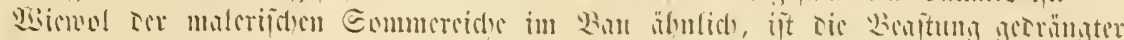

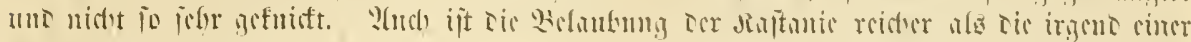
(sid)churt.

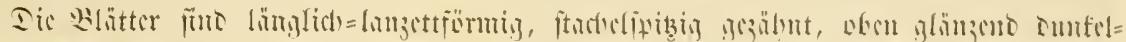

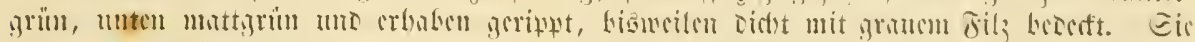

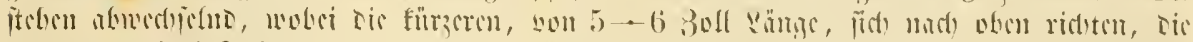

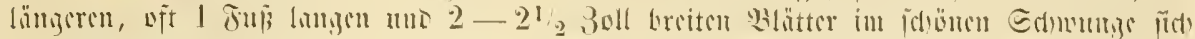
nacl matrin magen.

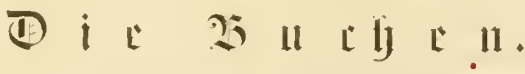

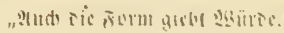 \\ (ํำ}

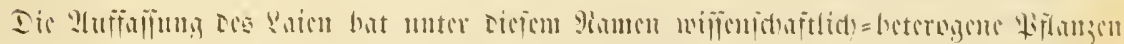

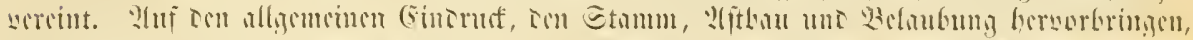

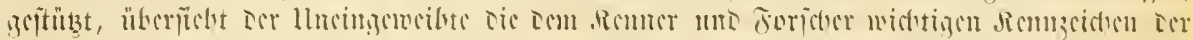

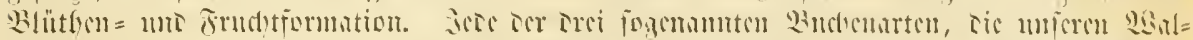

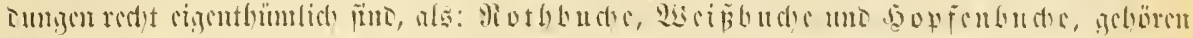

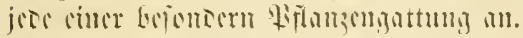

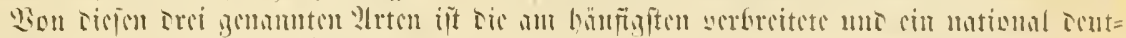
joter Rantm :

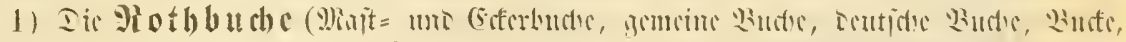

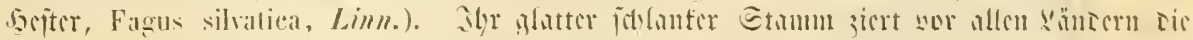

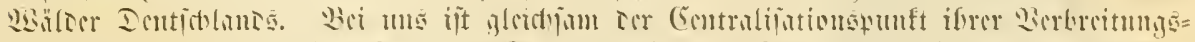

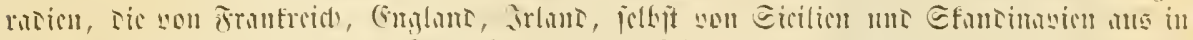

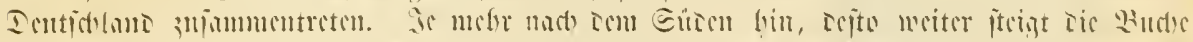
ant

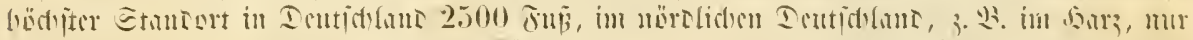

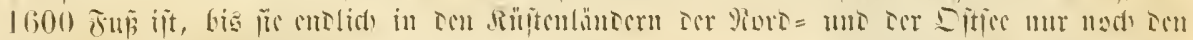

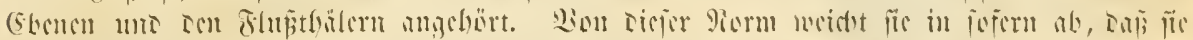

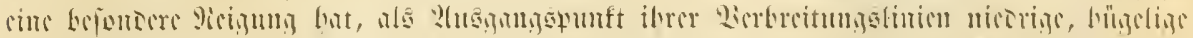
Worbrige ;

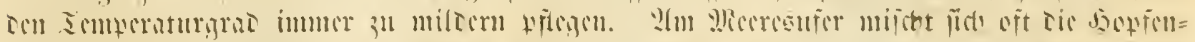

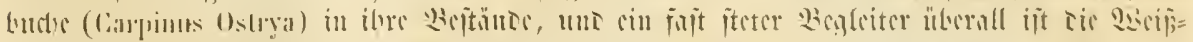

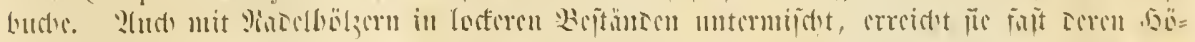

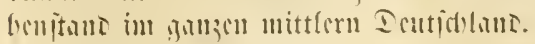

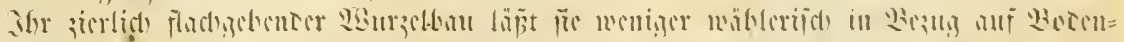

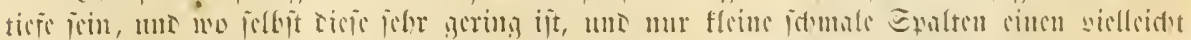

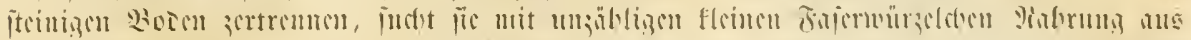

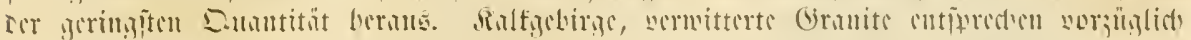

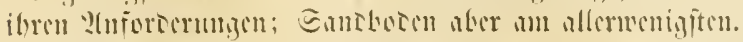

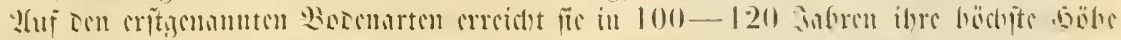

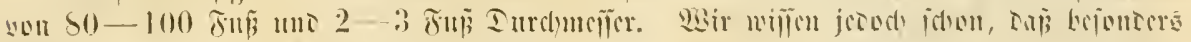

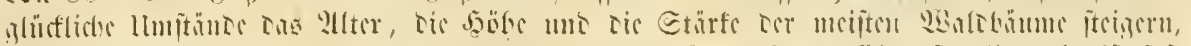

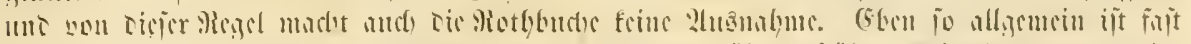

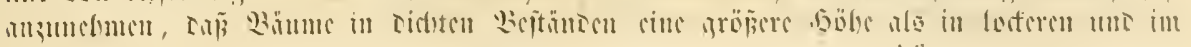




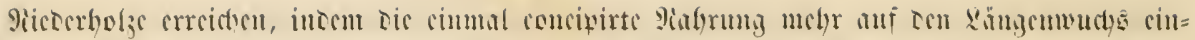

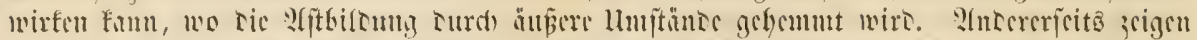

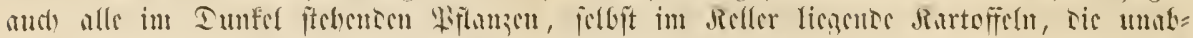

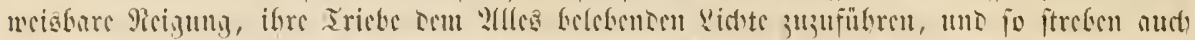

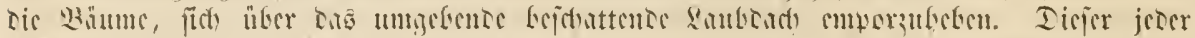

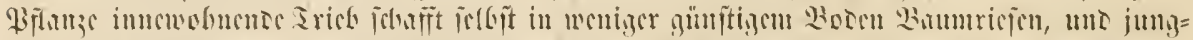

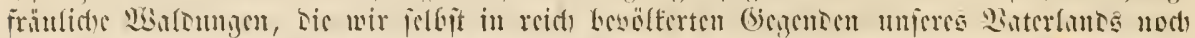

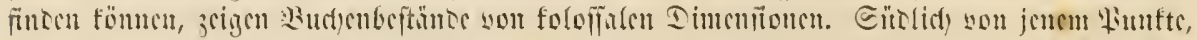

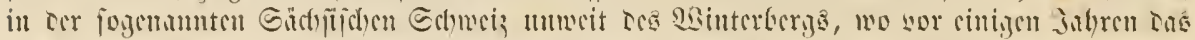

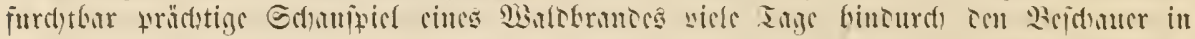

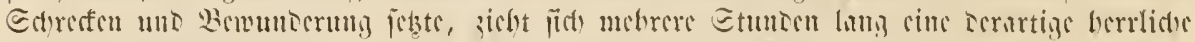

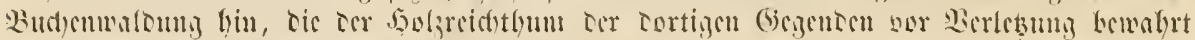

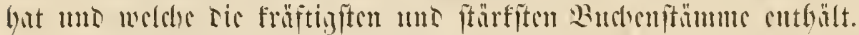

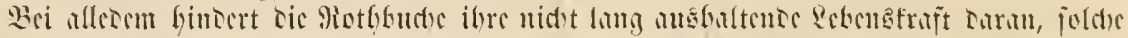

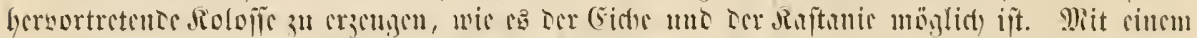

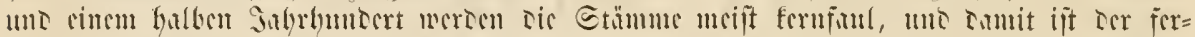

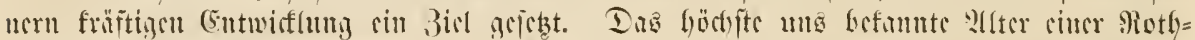

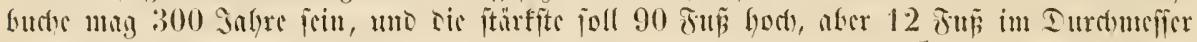

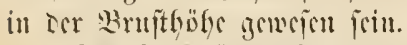

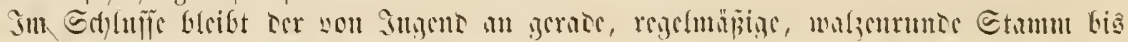

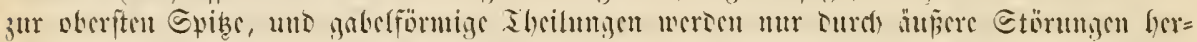

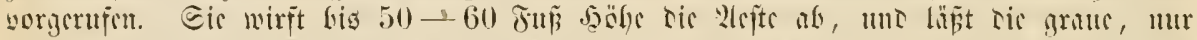

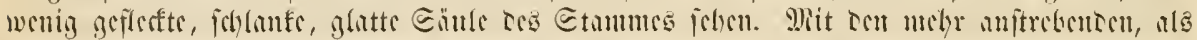

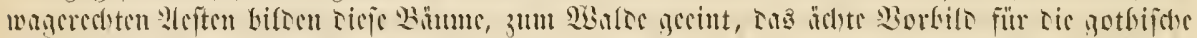

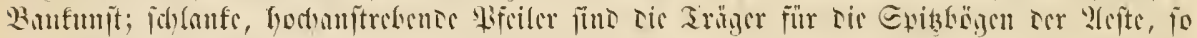

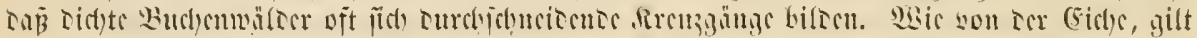

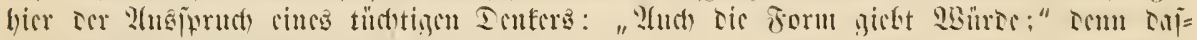

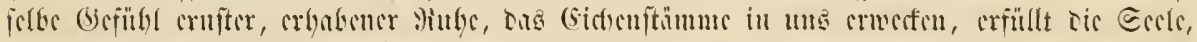
menu mix cincu Busthenom fetreten.

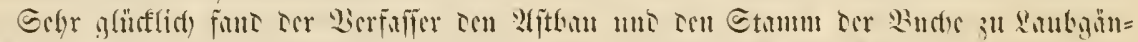

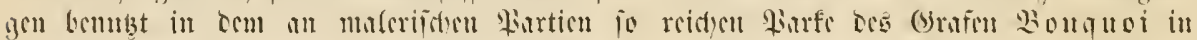

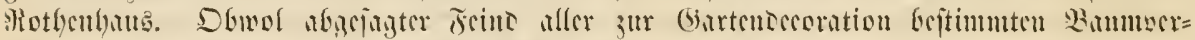

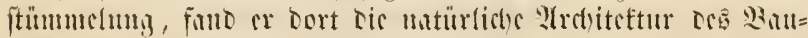

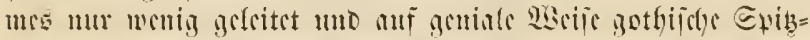

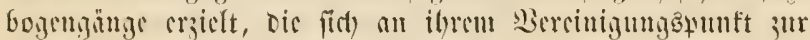
(id) önten tentublupel erfoben.

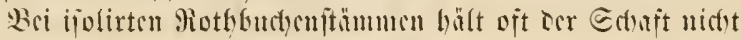

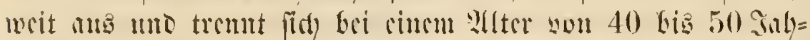

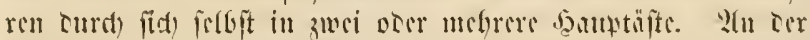

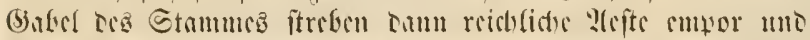

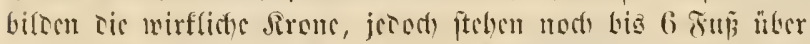

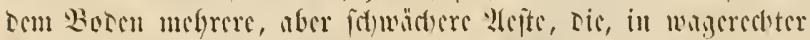

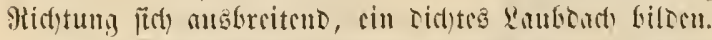

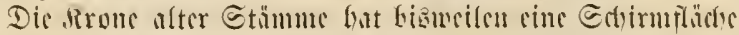

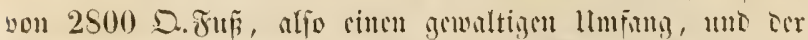

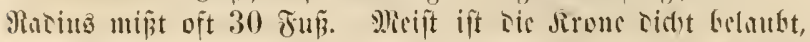

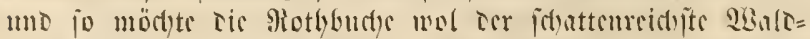

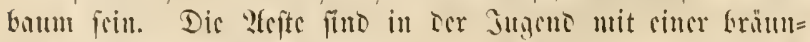

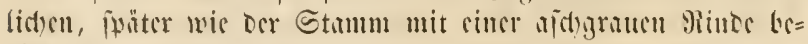
fleitet.

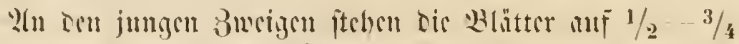
Boll limgen fomadsen Blattiticlen medifelitumoig uno jicmlioto

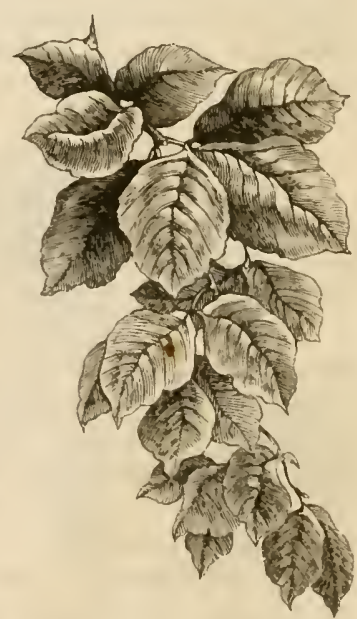

26. Saubyrawe oer Buthbute. 


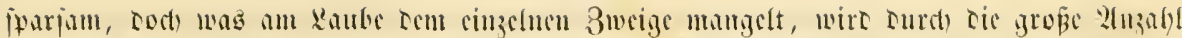

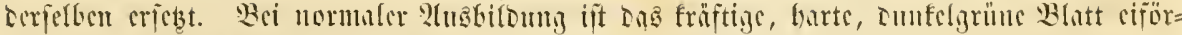

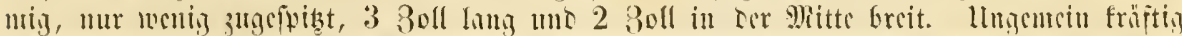

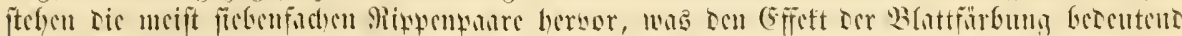

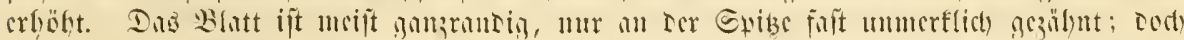

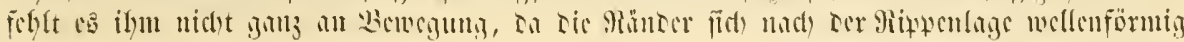
biegcn.

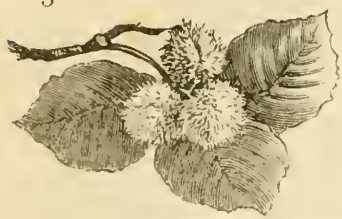

27. ซrritht rer Rotbuthe.

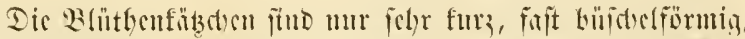

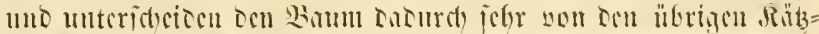

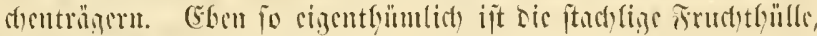

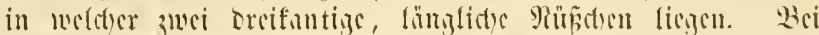

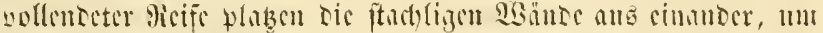

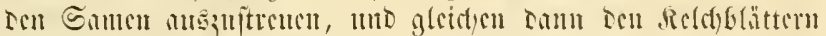
ciner 2 llumt.

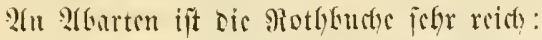

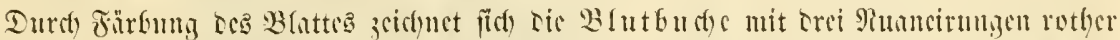

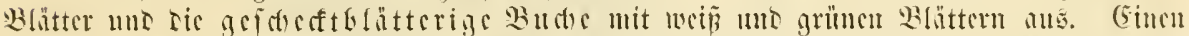

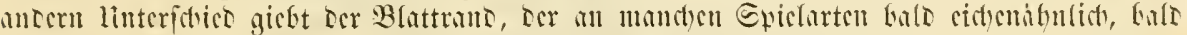

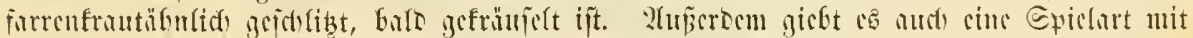

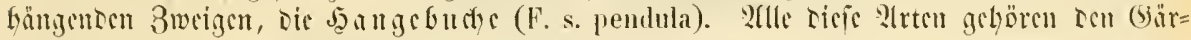
ten แแno Parfen an.

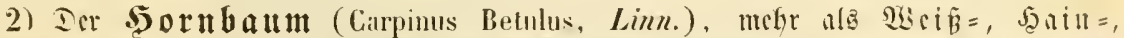

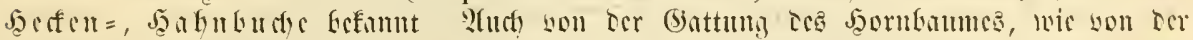

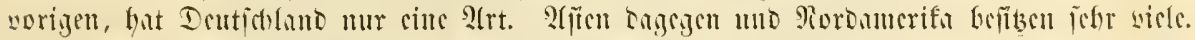

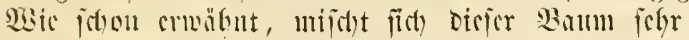

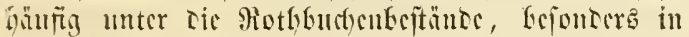

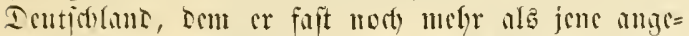

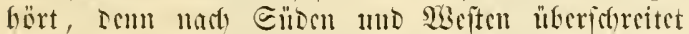

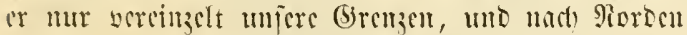
bin bleift or in Siormenen une Eroweren weit binter

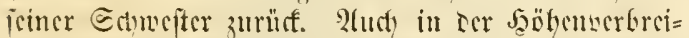

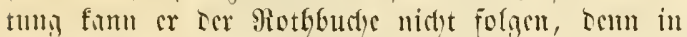
Scm mittlern Dentidylans ift frin föblifter Etansort

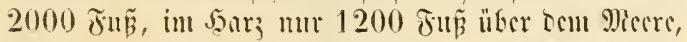

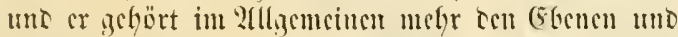

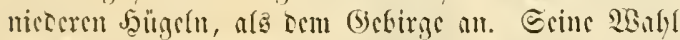

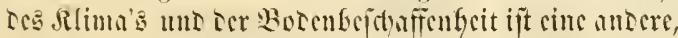

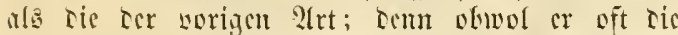

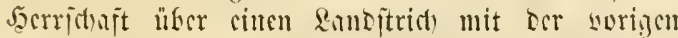

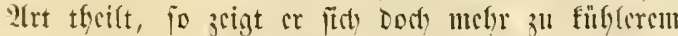

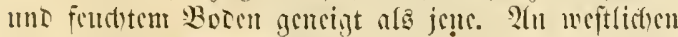

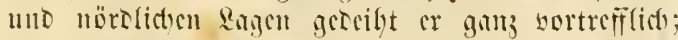

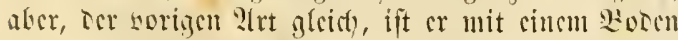
won geringer Ticfe zuriteoch. Iod) richtet or fict)

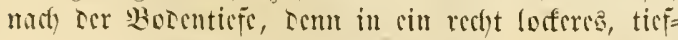

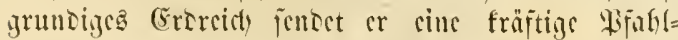
mutric!.

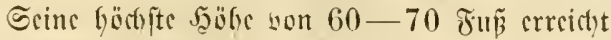

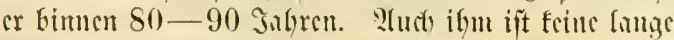

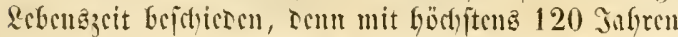

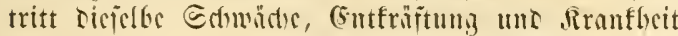

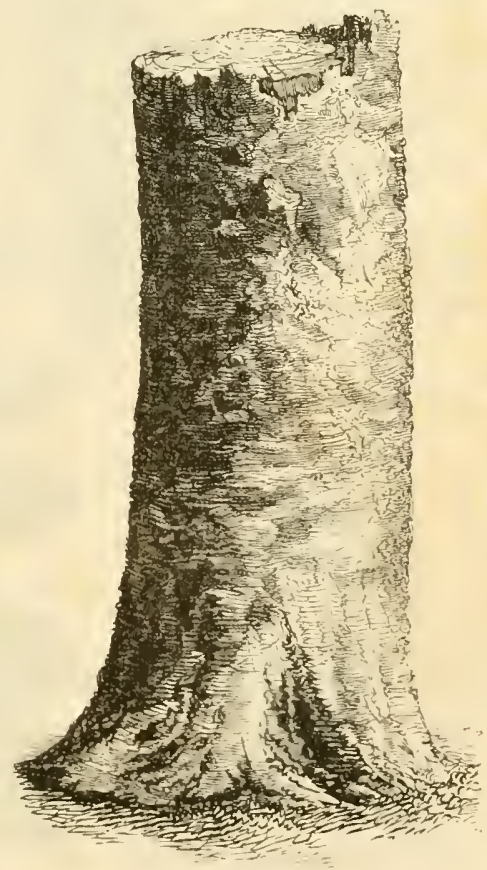

25. Elamm tor פgeifbude. 


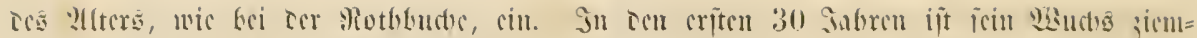

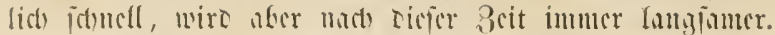

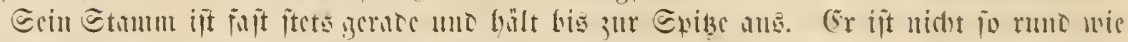

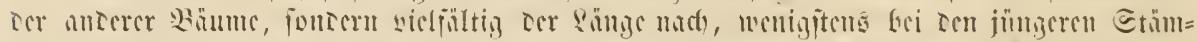

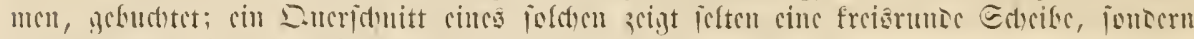
ijt buld onal, Galc firmpör= miz ber rums, mit cincr acer moberen ?(usturcitum= 3zen. Esten ift Ter Iurdumci=

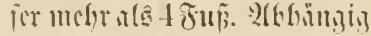
wou Ieu ridterm orer locfe=

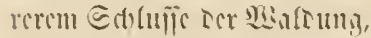

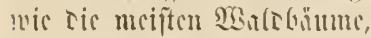
fogimnt for Jironentsul foi ijolirten Simsisisusn in sincr

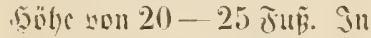
rincu 2Binfel woll 20-30

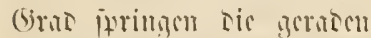

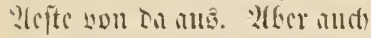
fier funo Dis ticferen sfocile

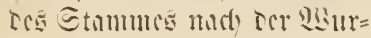
id 3u icmlion meit unten nod) mit jomüderen, meijt

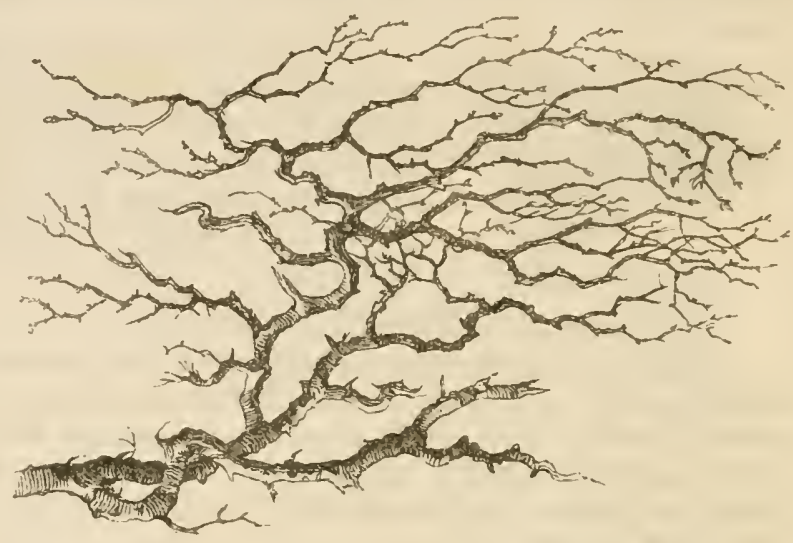

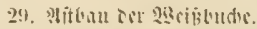

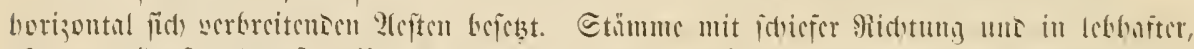

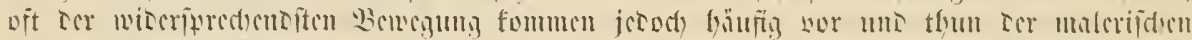

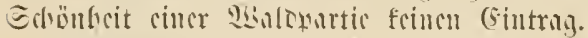

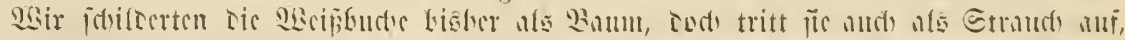

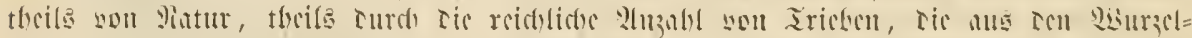

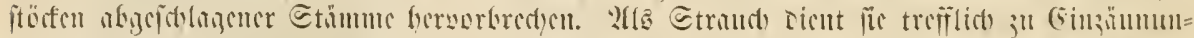

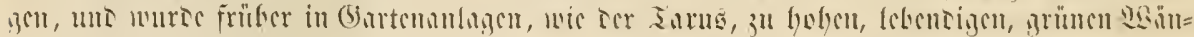
Iell fermutgt.

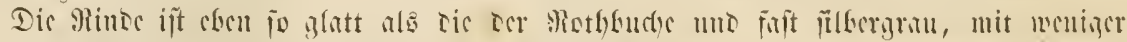

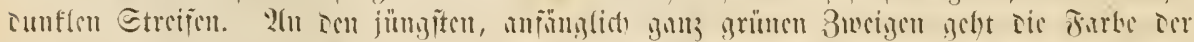

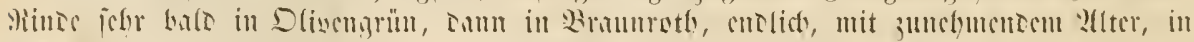

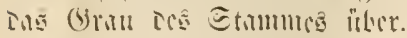

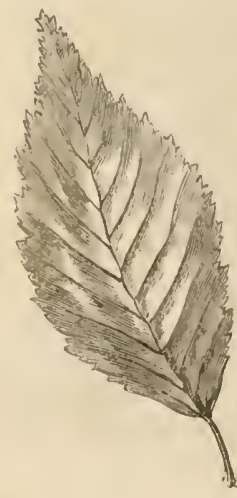

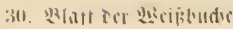

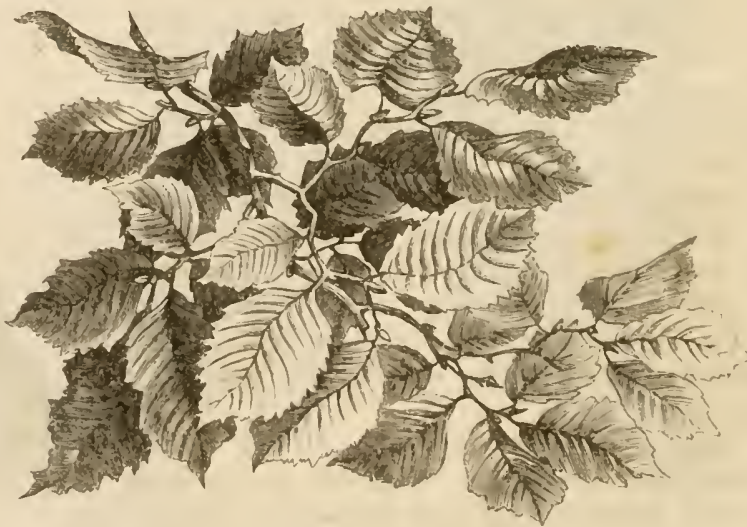

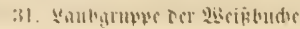




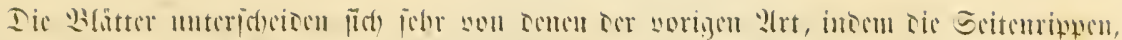

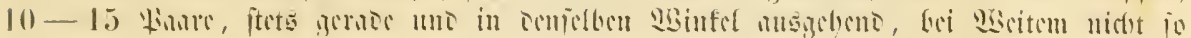

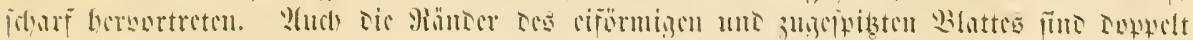

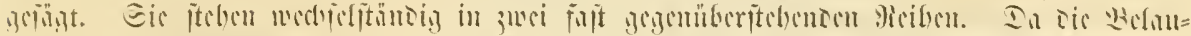

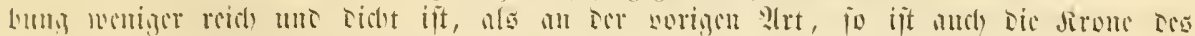

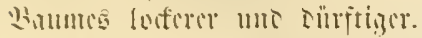

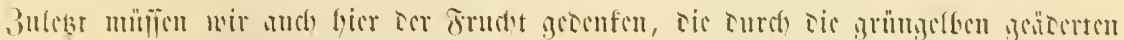

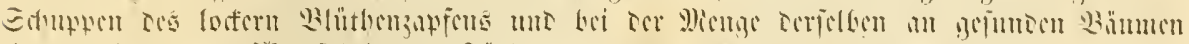

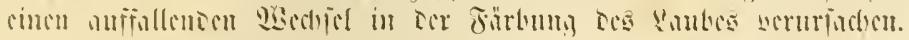

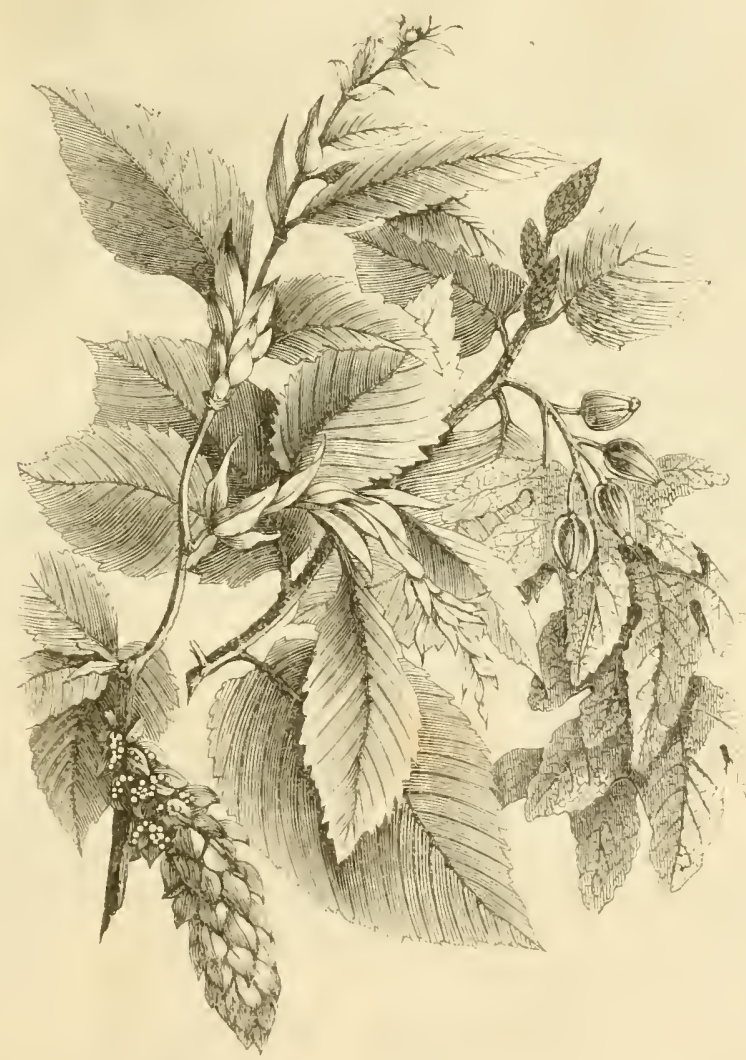

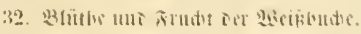

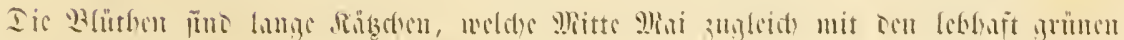

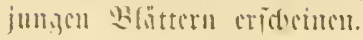

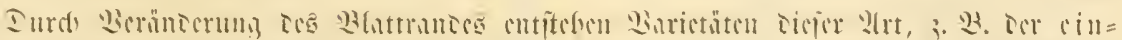

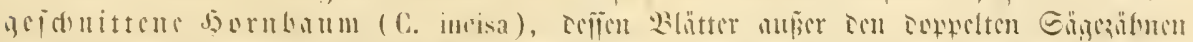

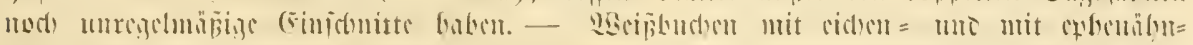

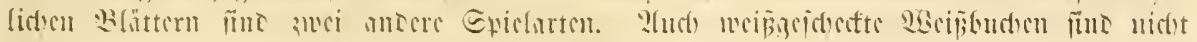
jeltert. 


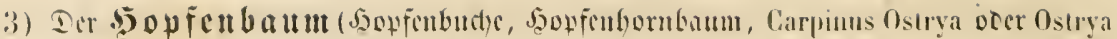

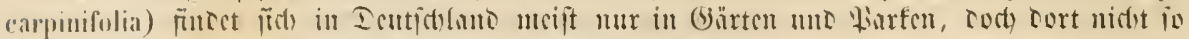

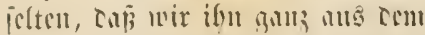

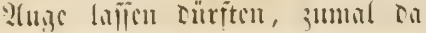

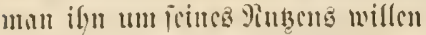
unter sic 3 abl ter gisulebüume

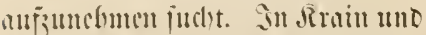

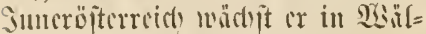

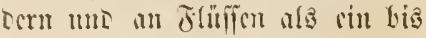

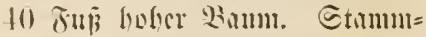

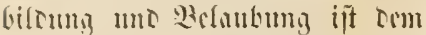
Ter Misciphuste völlig älonlict. 9ur

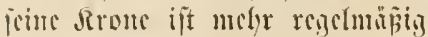
uno feigelförmiz. Die Befitter puno

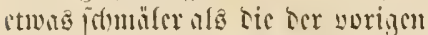
Itrt, jonjt chou io gezühnt. Dor grö̈itelluterictico liegt inter Form Eer weiblisten : Blïtfe u. our torran

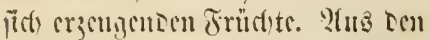
Estumen ter weiblidyen Belütben crmadjen grö̈̈ere, bhijenartige, geripute llmbullumign, metede ie

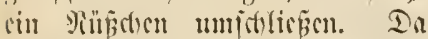

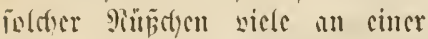

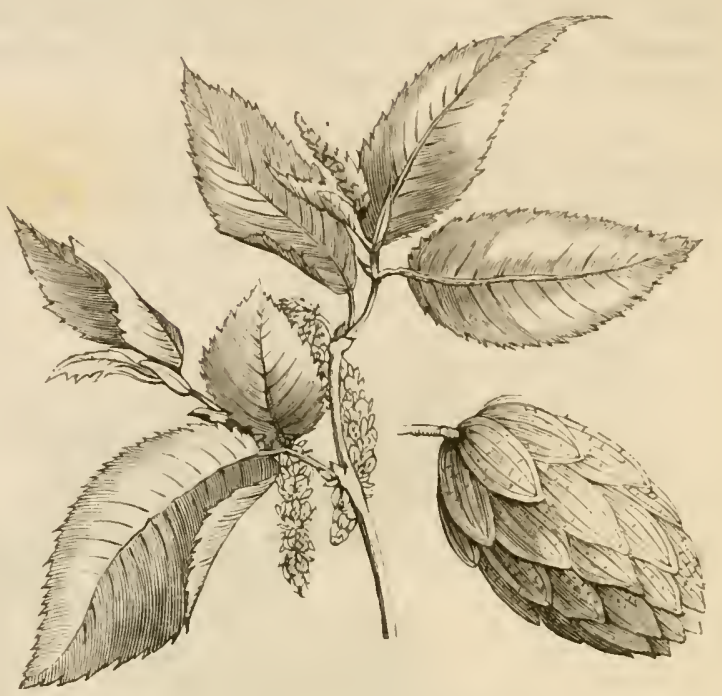

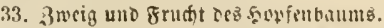
Eximos, unis dis Estupen tor

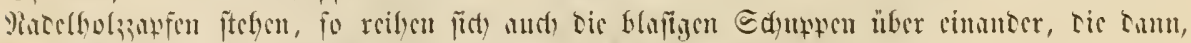

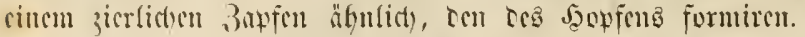

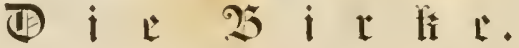

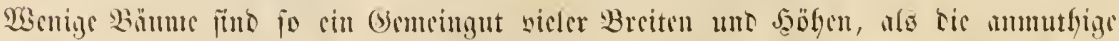

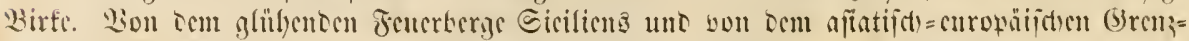

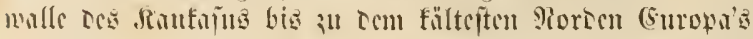

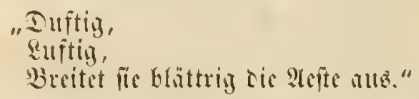

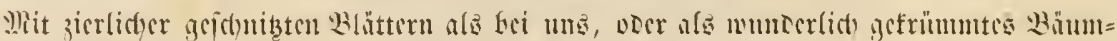

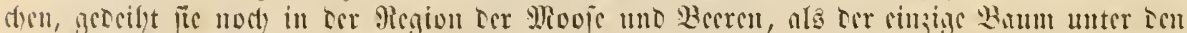

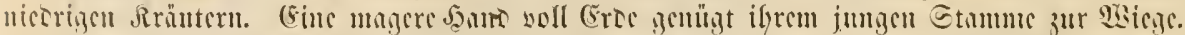

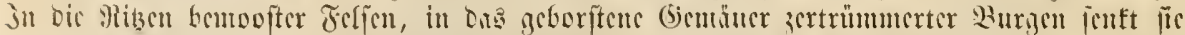

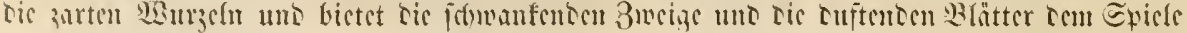
DCa isintea car.

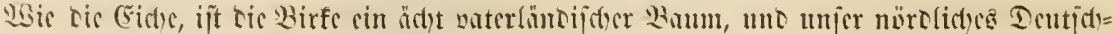

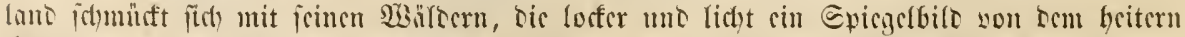

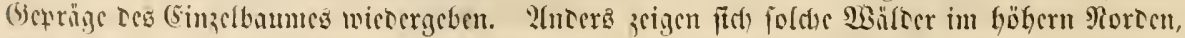

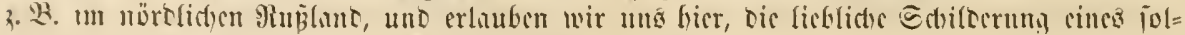

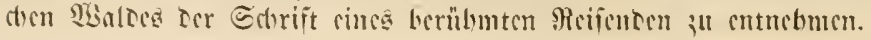




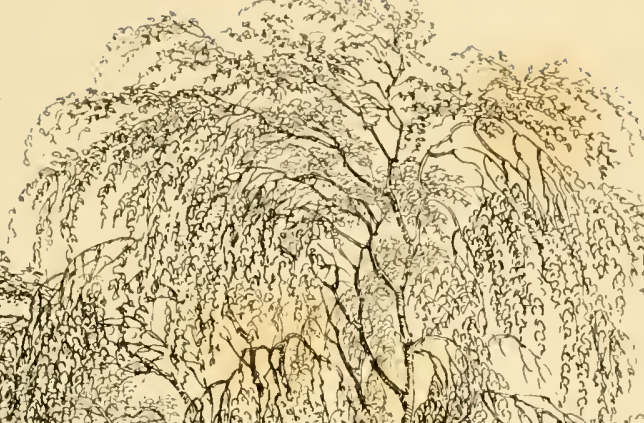

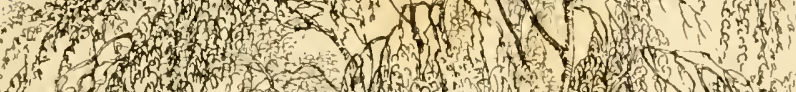

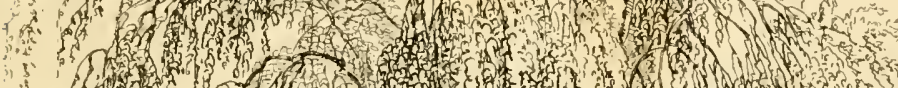

solom

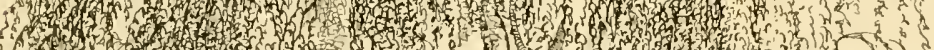
(n)

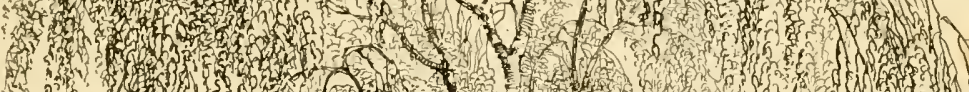

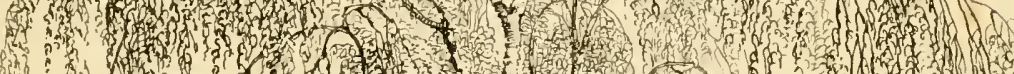
3h? 6.

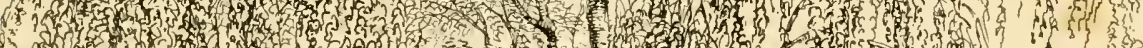
9.

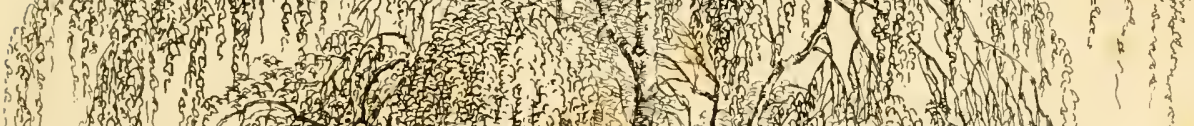

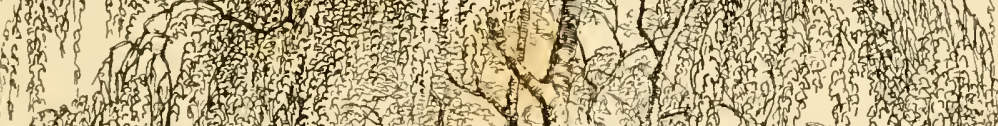

19.5.

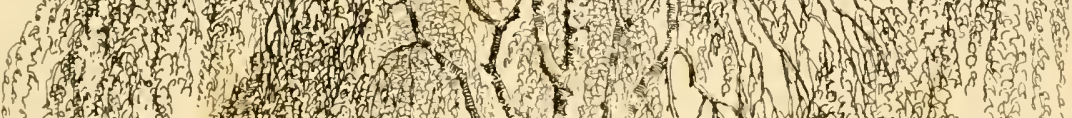
s?

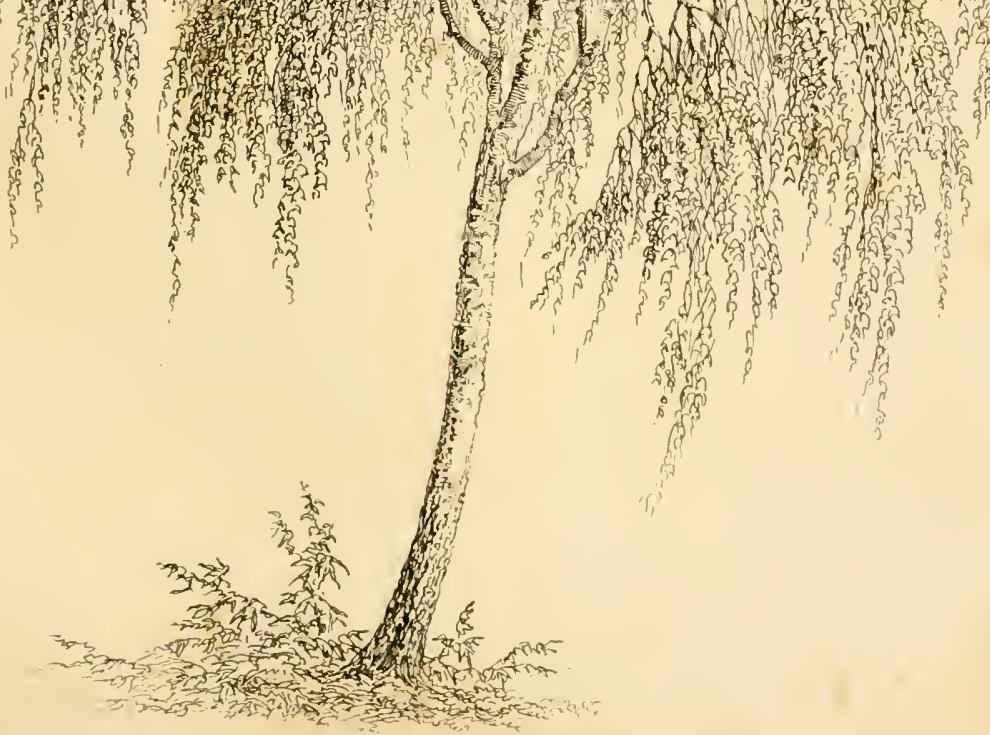





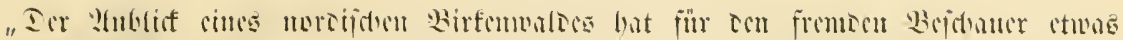

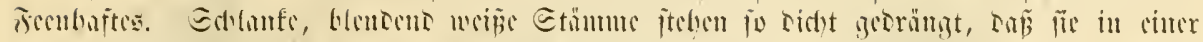

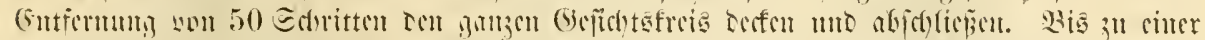

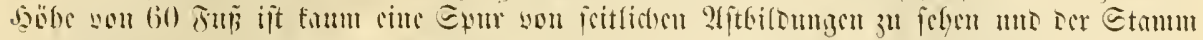

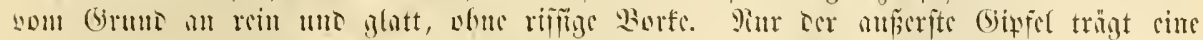

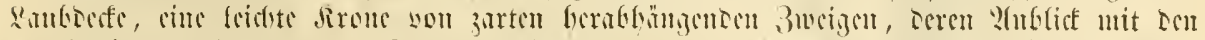

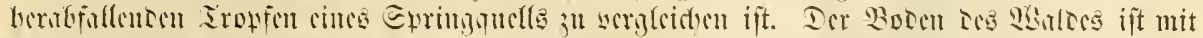

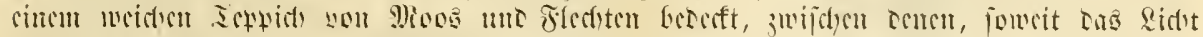

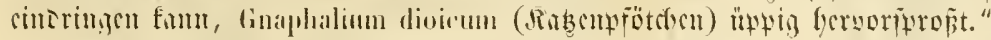

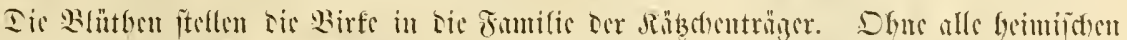

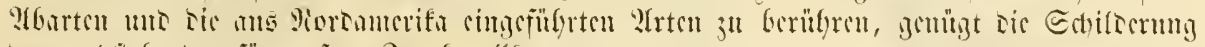

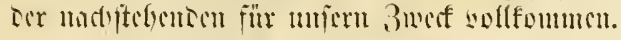

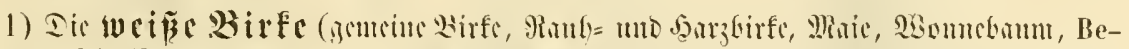

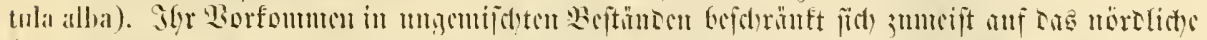

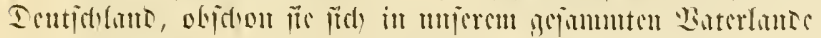

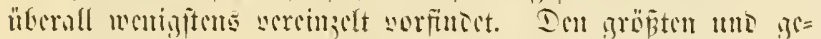

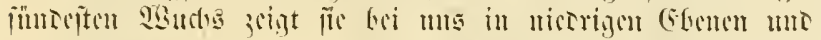

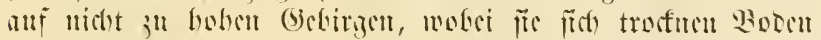

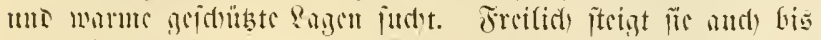

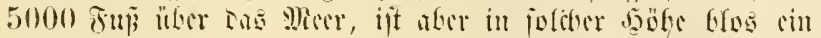

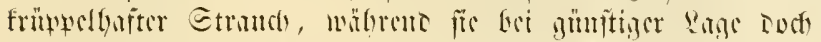

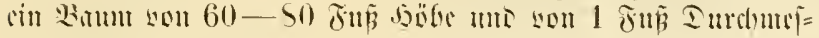
irr wirt.

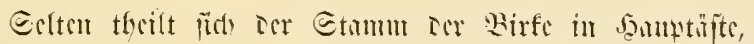

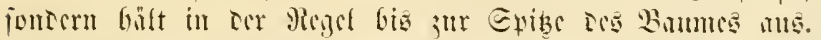

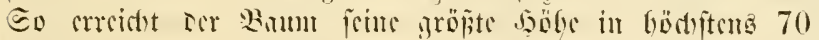
jituren.

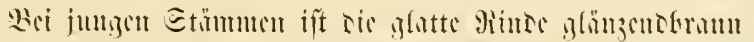

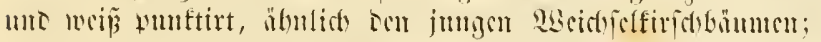

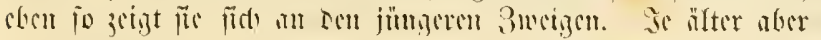

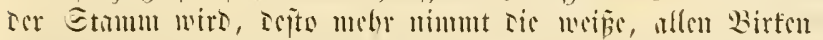

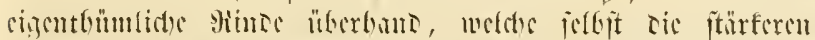

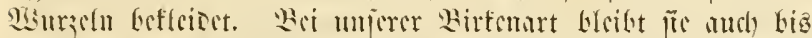

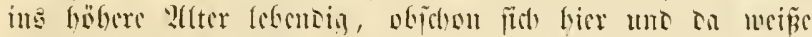

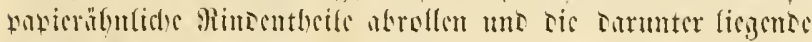
Grame Rorfrime itart aufript.

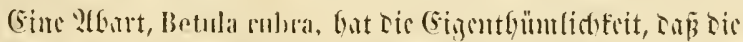

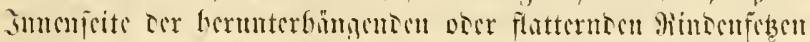

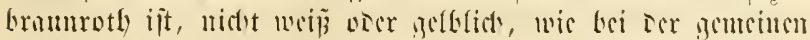

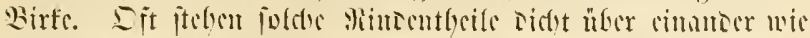
Eิt)

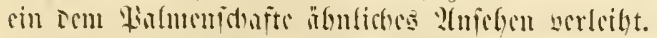

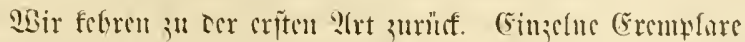

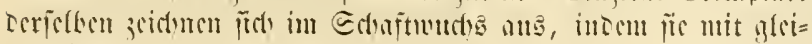

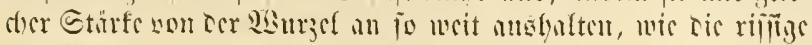

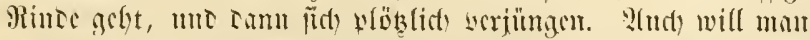

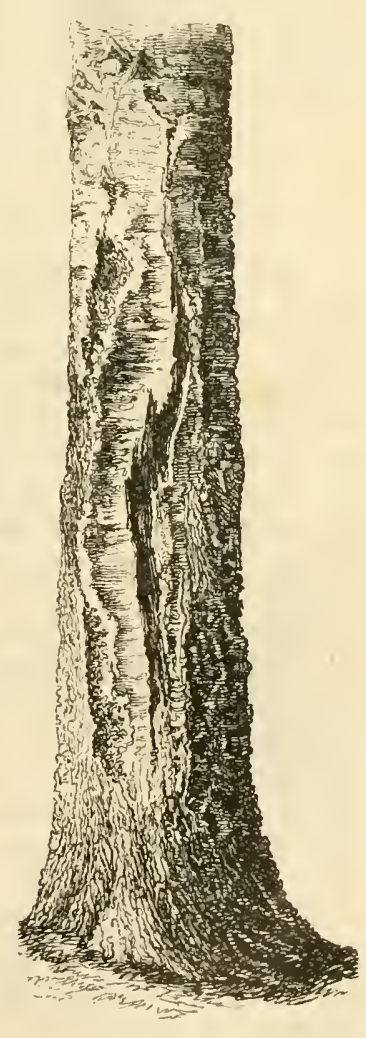

34. Etanum Der Bitfe.

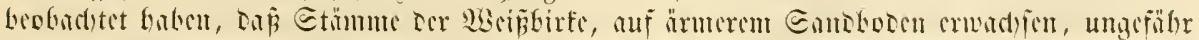

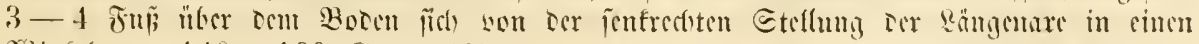

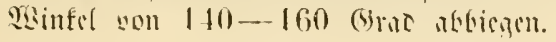




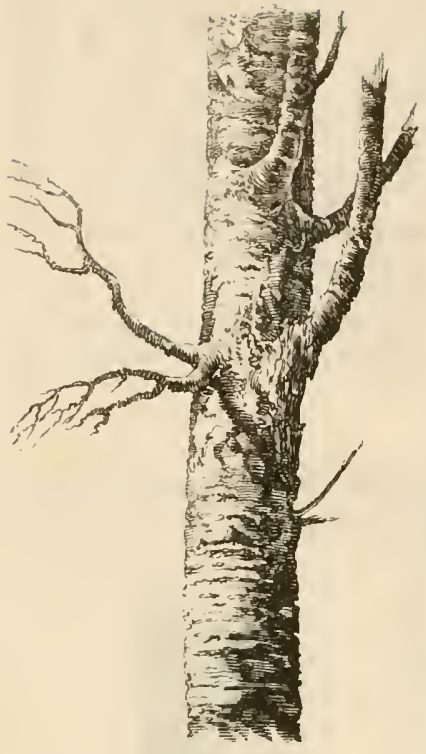

35. Mhettelitinum tor girfe.

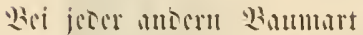

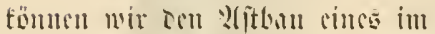

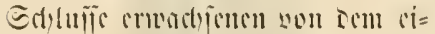

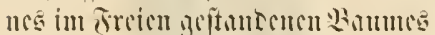

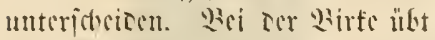

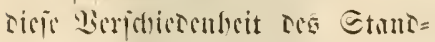
orta feincu oocr fidor acrimigen

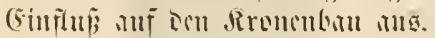

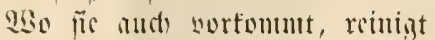

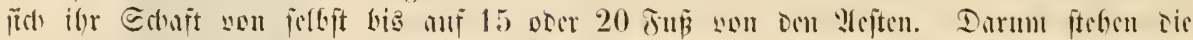

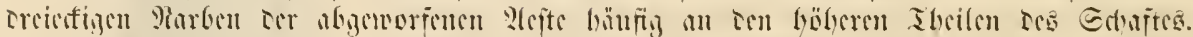

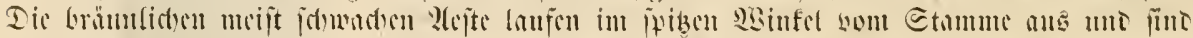

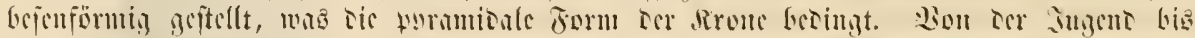

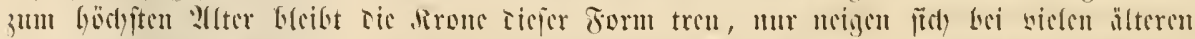

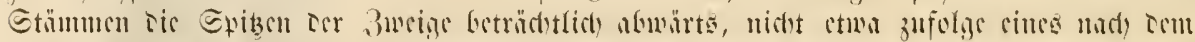

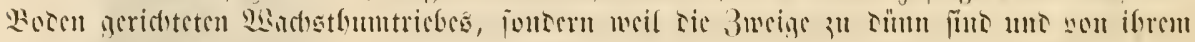

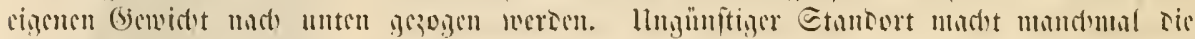

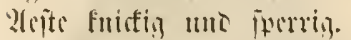

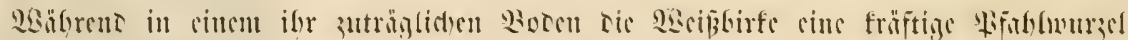

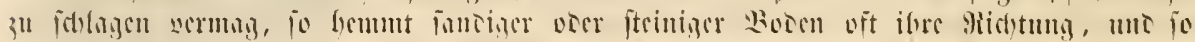

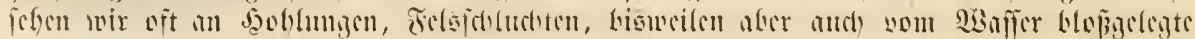

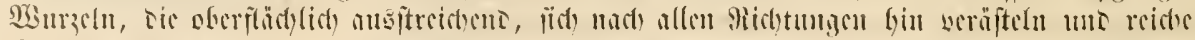
Eproitcm trifin.

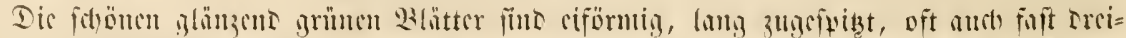

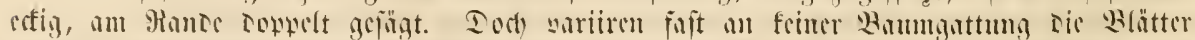

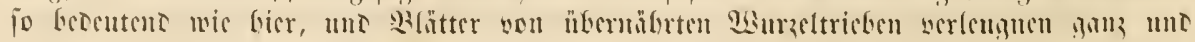
gon ifore Sycrfunit. 


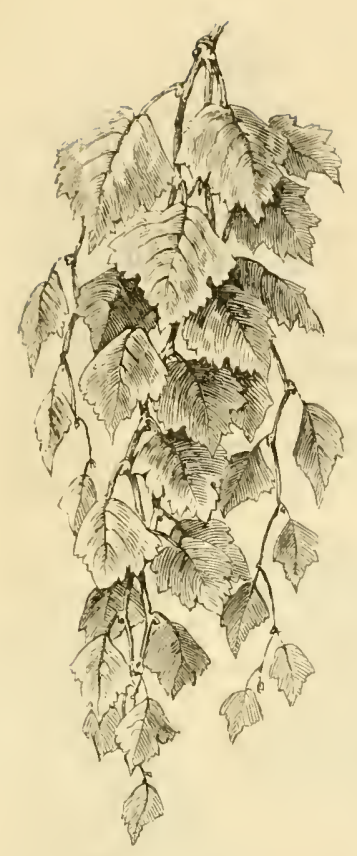

37. 3uelglauf und ตBlatfitand on sBrişuirf.

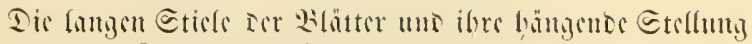

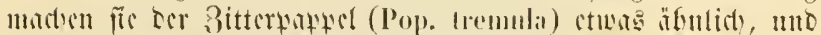

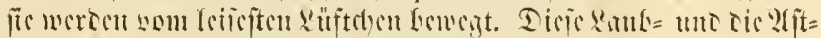

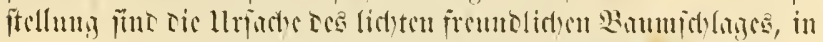

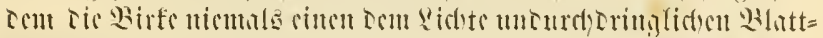

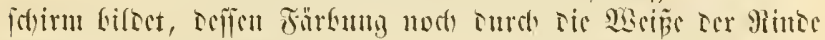
becontento erfüt wirt.

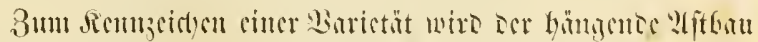
bei oer ₹runcr = voor dängebirfe (Betula pendula). Dis

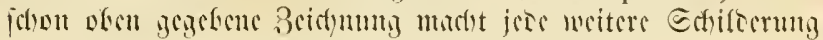

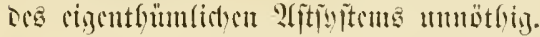

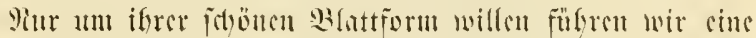

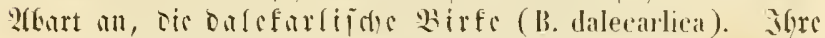

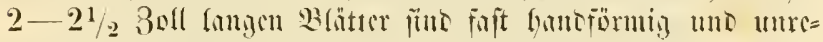

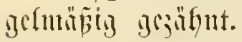

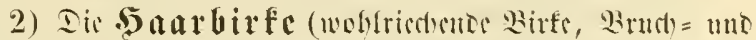

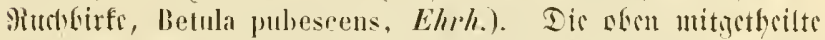

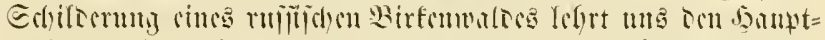

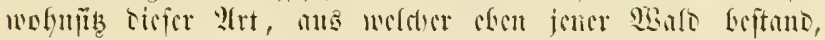

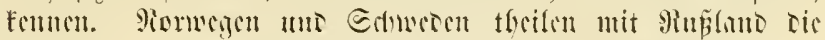

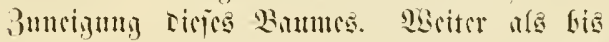
50 (S)no nat) Sëtom fin cristeint or nistt in

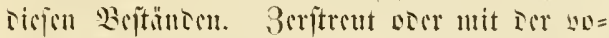

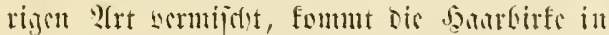

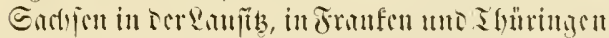

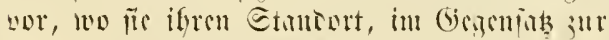

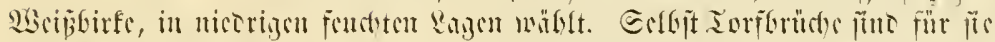

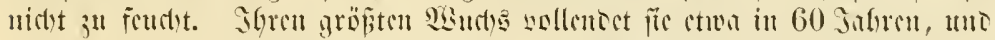

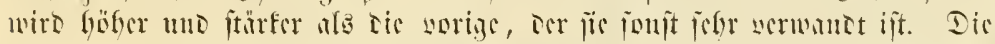

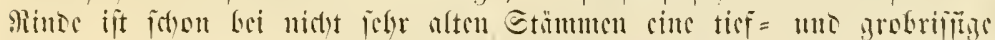

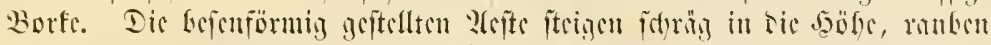

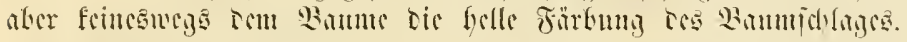

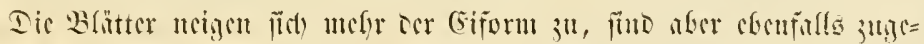

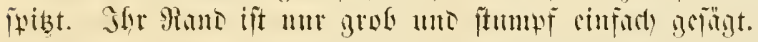

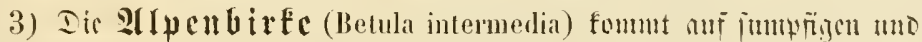

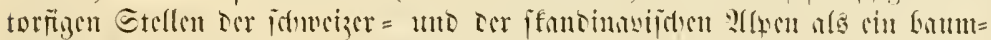

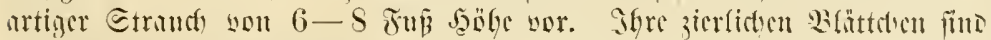

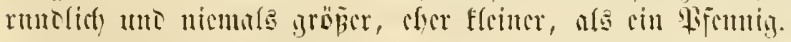

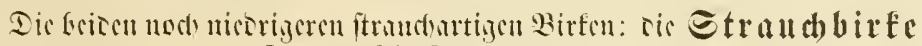

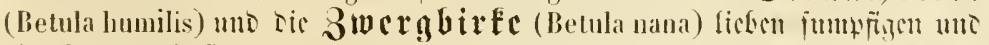

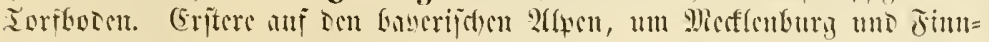

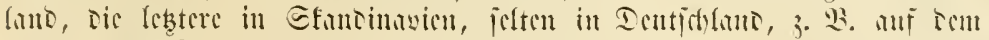

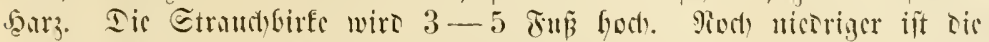

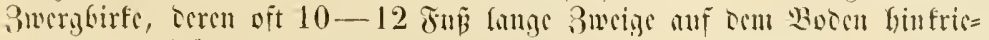

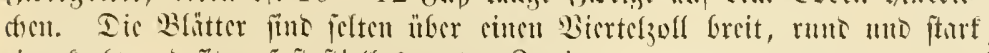

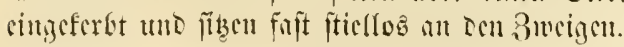

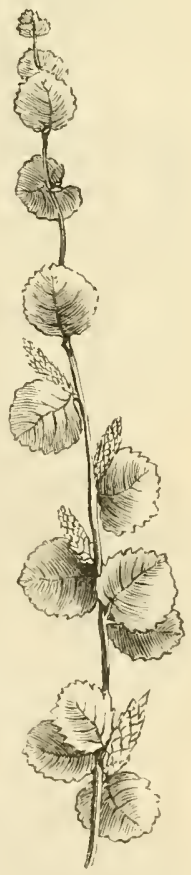

35. 3ueig tor 3 nerg: birfe 


\section{(1) $\mathfrak{i} \mathfrak{e}$}

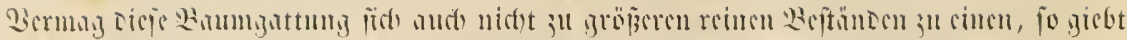

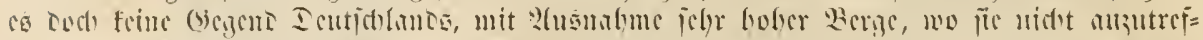

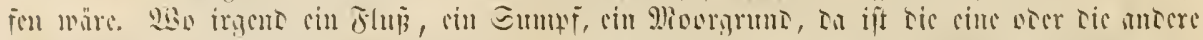

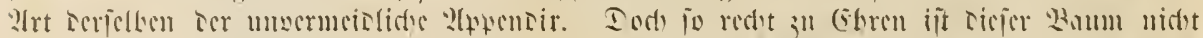

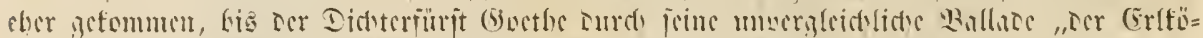

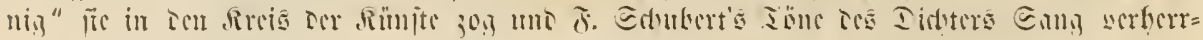
lictem ums seremigfen.

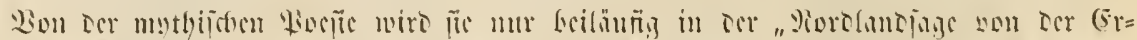

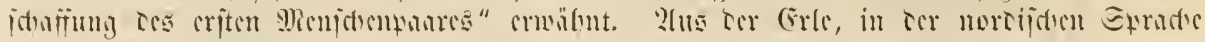

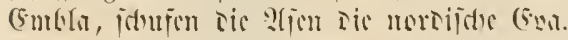

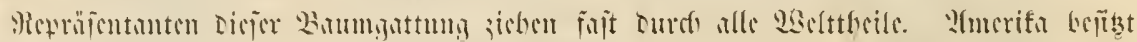

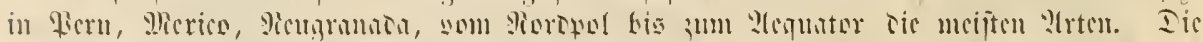

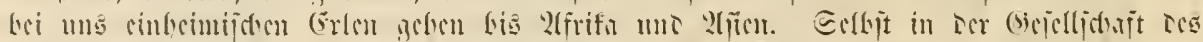

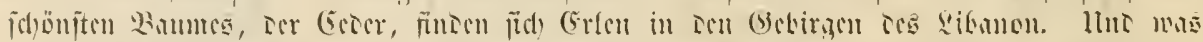

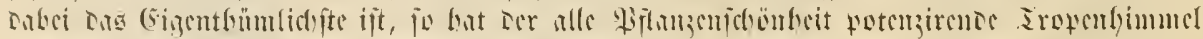

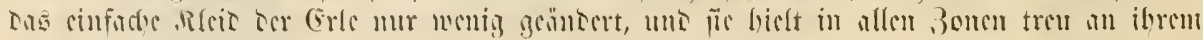

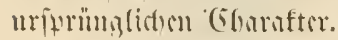

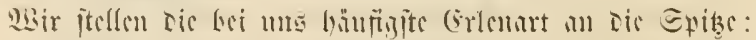

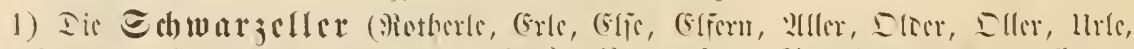

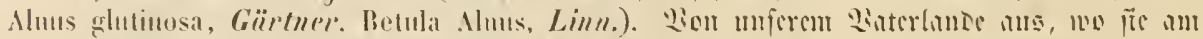

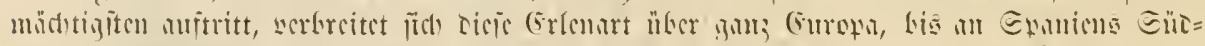

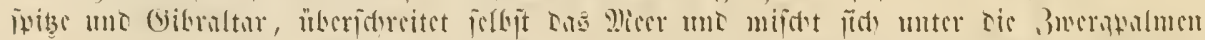

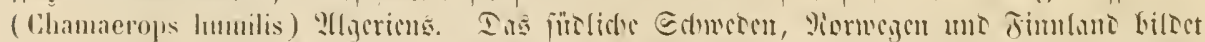

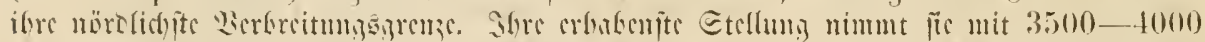

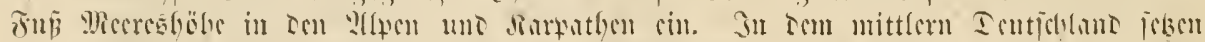

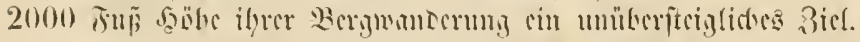

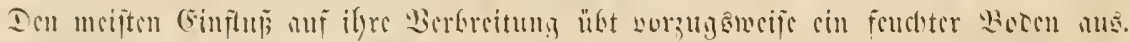

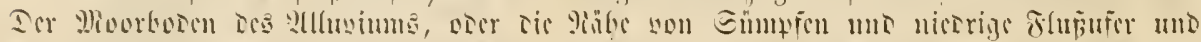

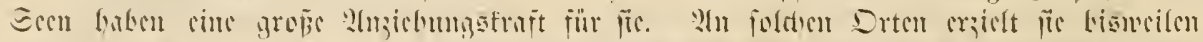

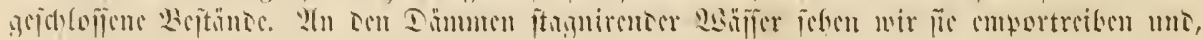

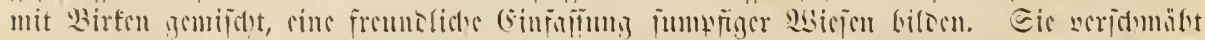

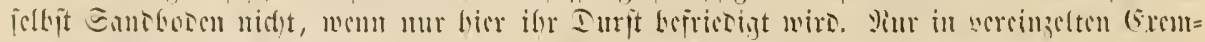

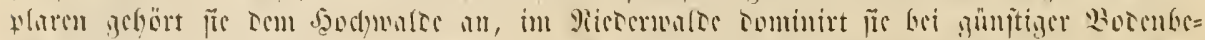

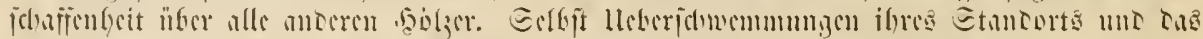

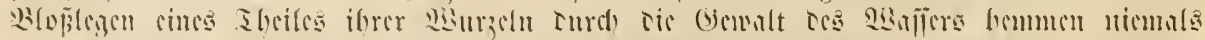

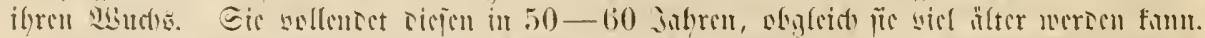

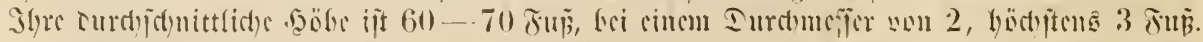

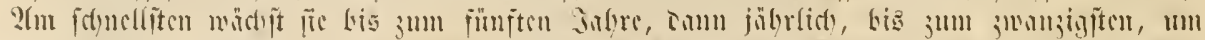

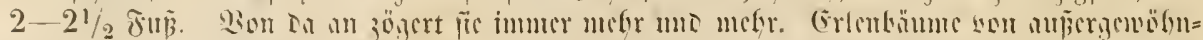

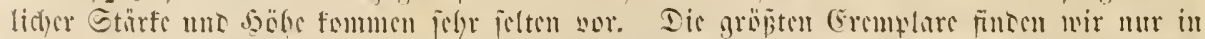

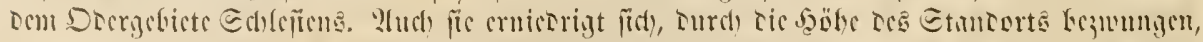

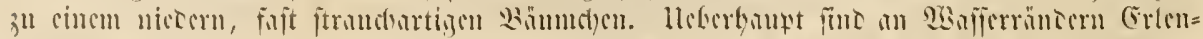

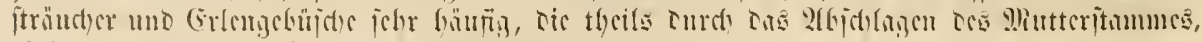

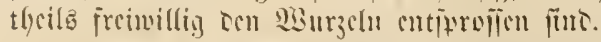

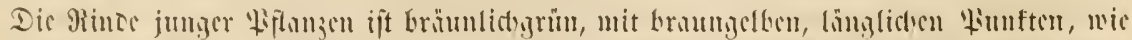

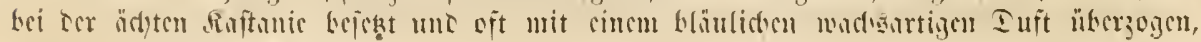





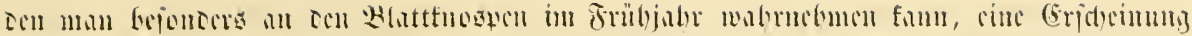

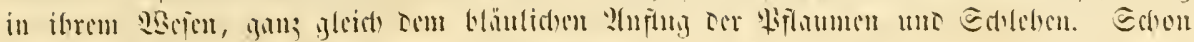

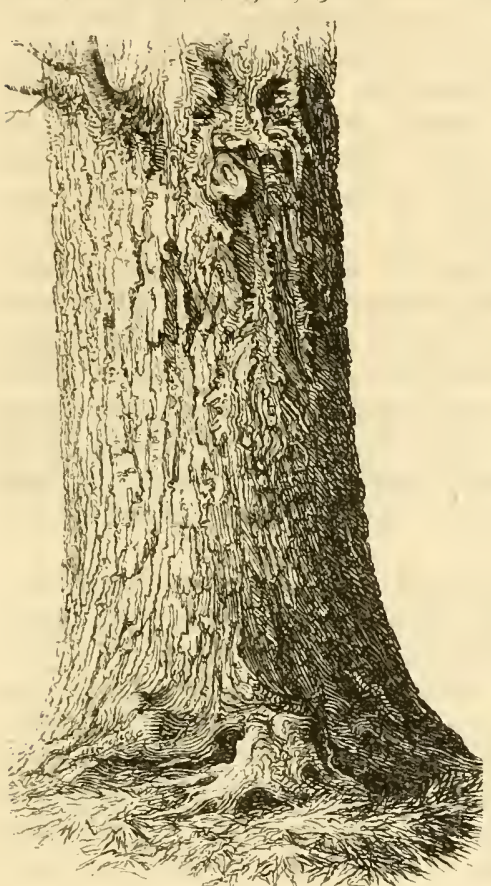

39. Etımun oor Erts.

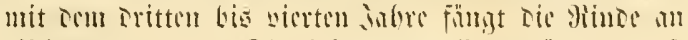

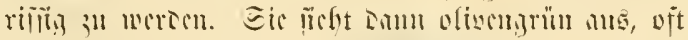

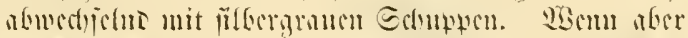

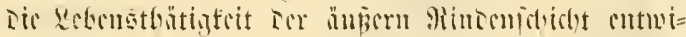

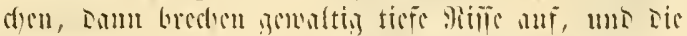

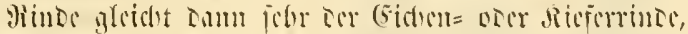

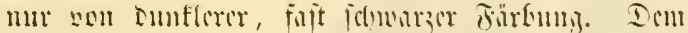

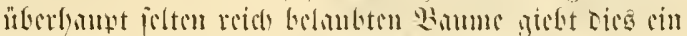
ringteres : ?lusichor.

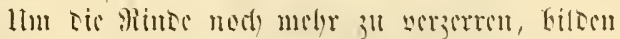

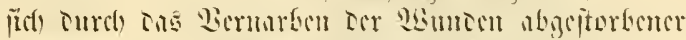

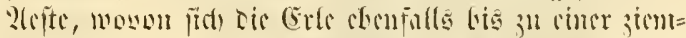

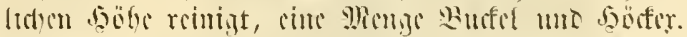

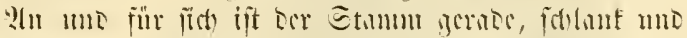

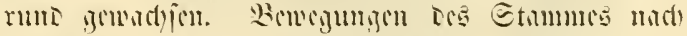

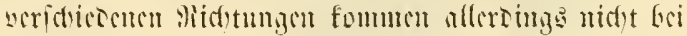

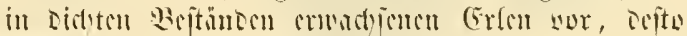

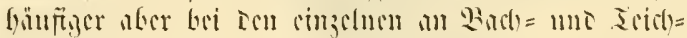

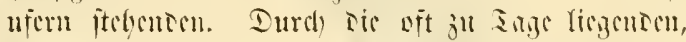

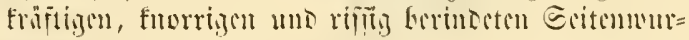

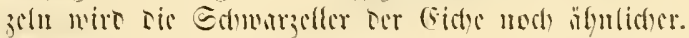

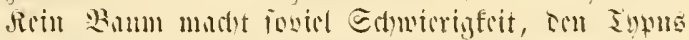

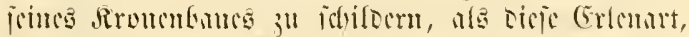

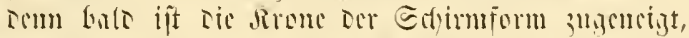

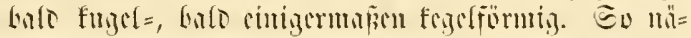

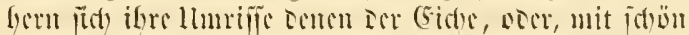

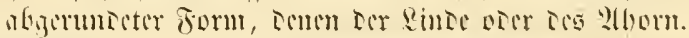

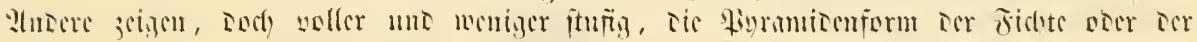

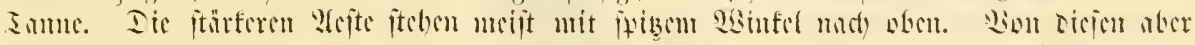

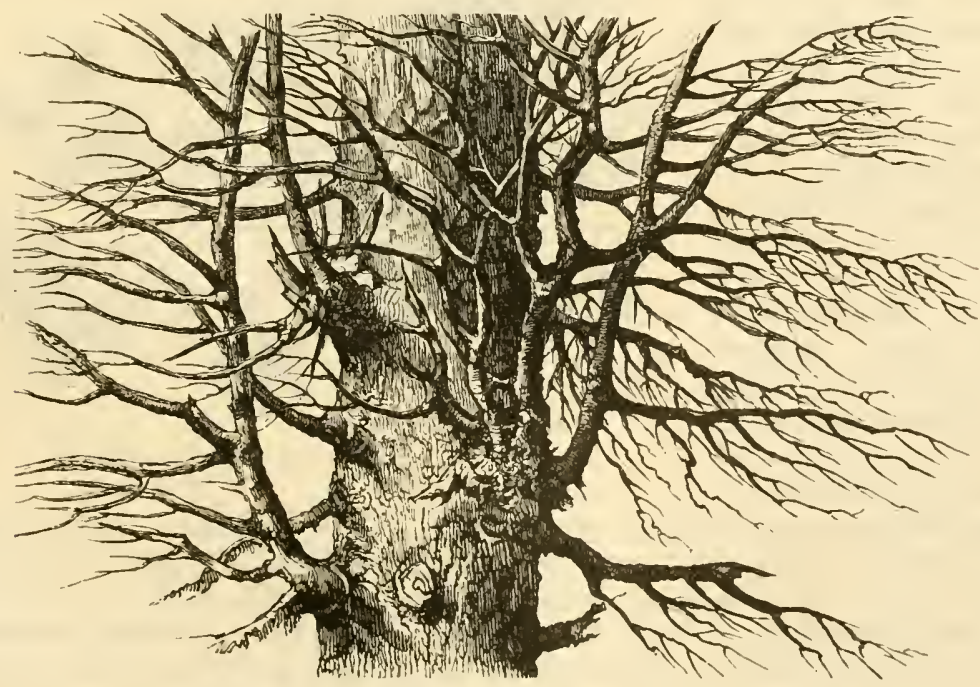

10. Mitciut $\mathrm{ret}(6 \mathrm{rlc}$. 


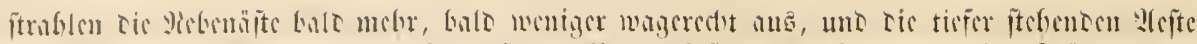

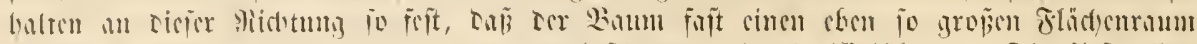

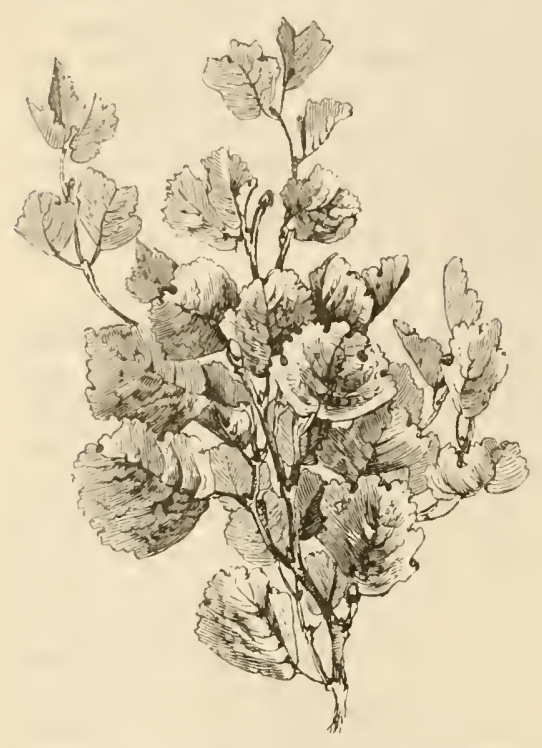

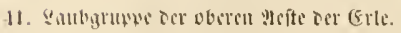

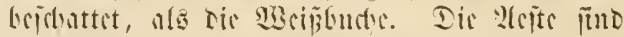

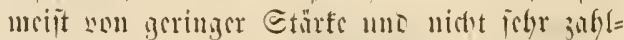
reide, woourch tic Edmwericrle, trob ifres

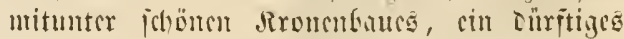

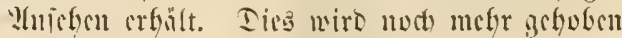

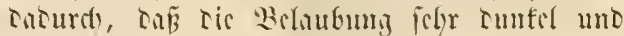
nisht reichlide iit.

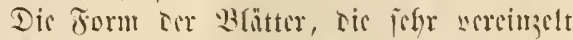
mest) beift, tir Exibe cor (Siform ift tom Etiele jubz= want. Weri jumben fröftizon Sristen ift oor

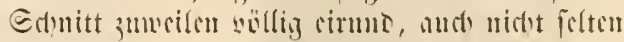

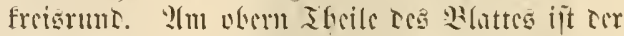

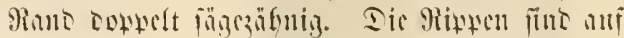

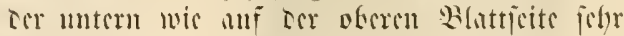
mirfirt.

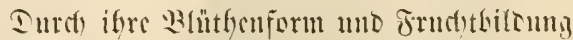

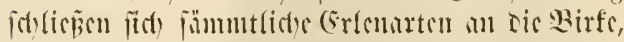
als birfenfrüst)tige Räb(t)

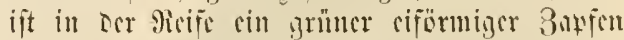

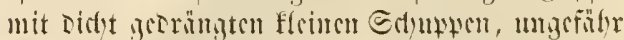

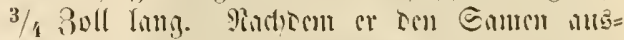

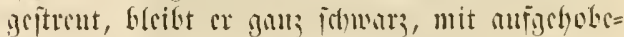
ner Eolutwen, nod im undtiten subre an tom

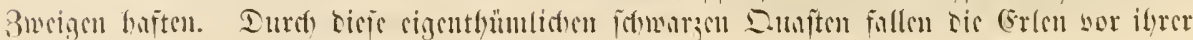

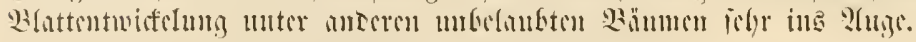

Lion meloren Exiclar=

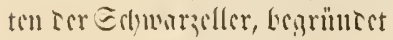

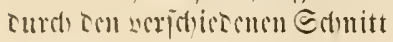

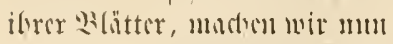

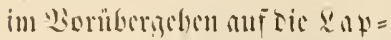
foucllor (Almus laciniata) munuerfoum, coren 3iculich

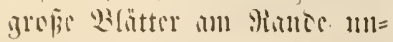

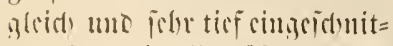

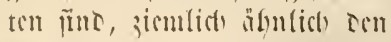
(simburbtumger for (Fid)en= blättri.

\section{2) Iir 2 secibellet} (nortijde (sller, Esmucrle, Alus incanas ) ift in Iontict)= lant bri gsertem midet io ge=

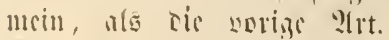

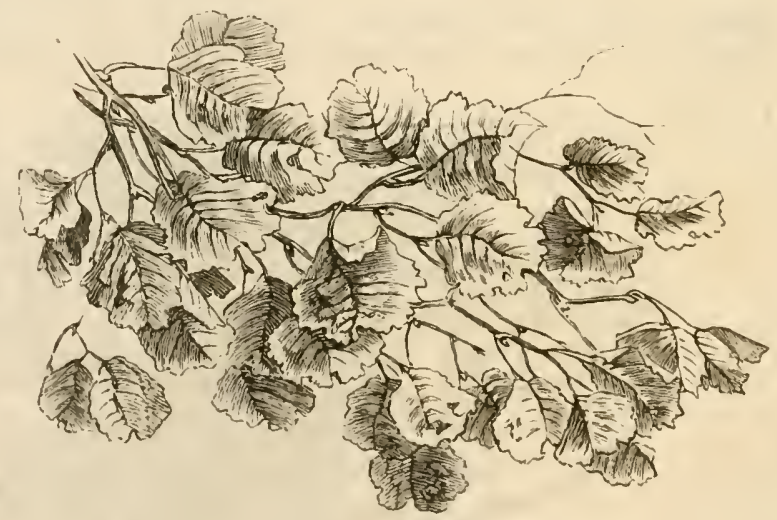

42. 8aแ

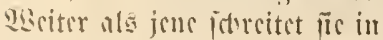

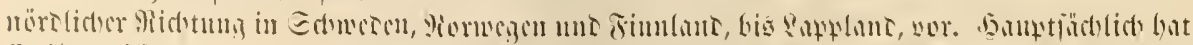

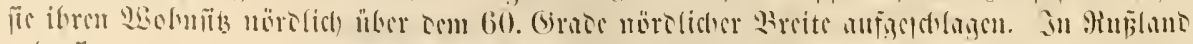

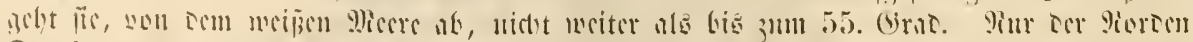

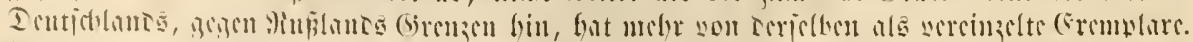

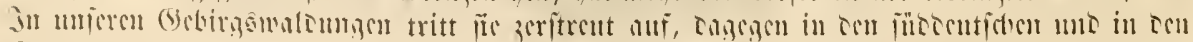

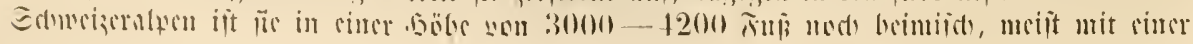





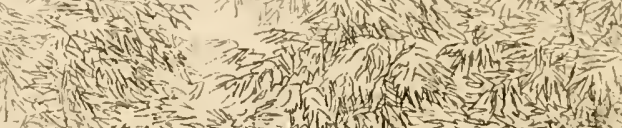

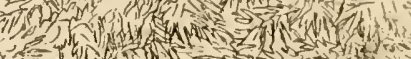

r.t.

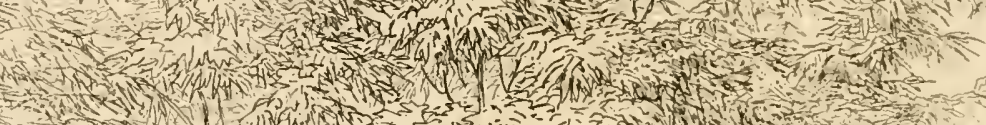

- $5-1$.

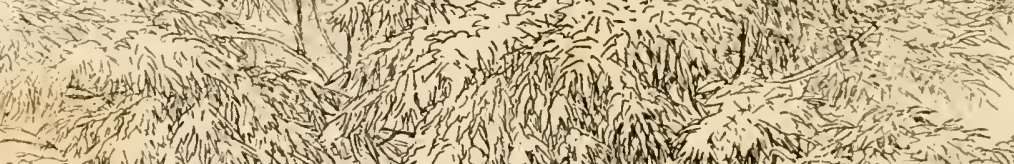

3.m.

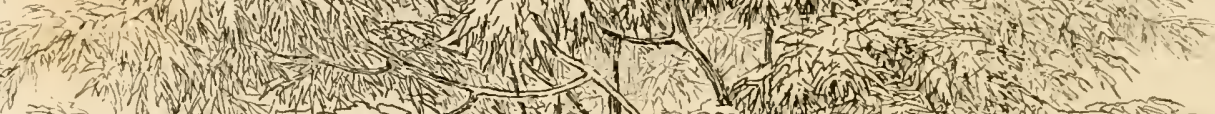

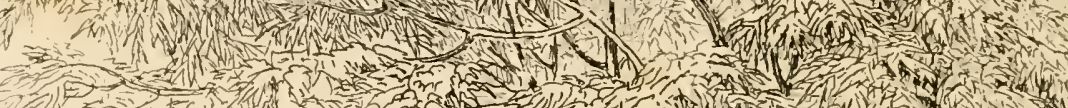

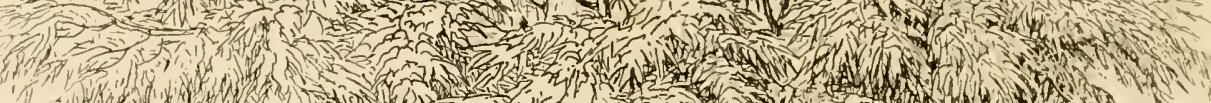

）

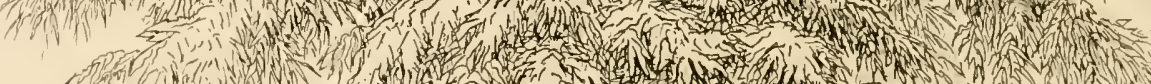

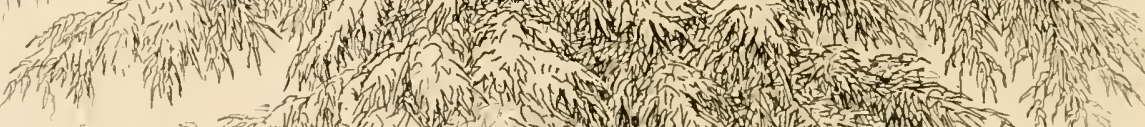
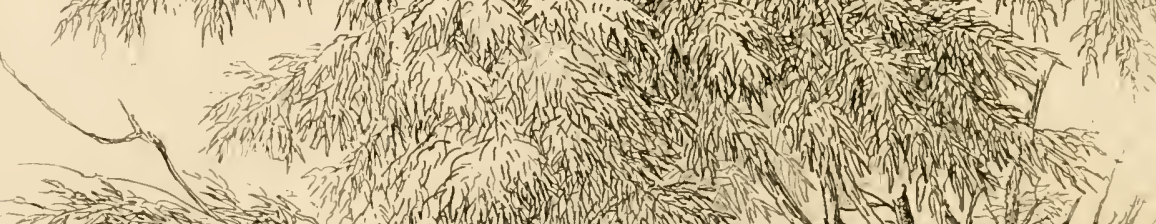

Nis.

(1)

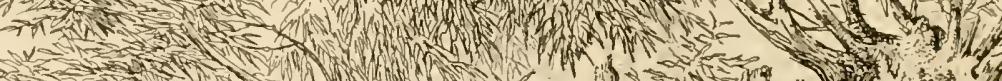

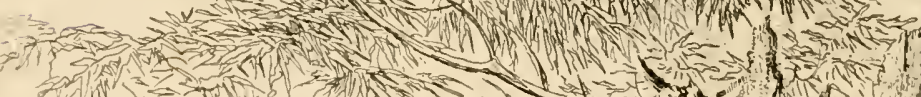

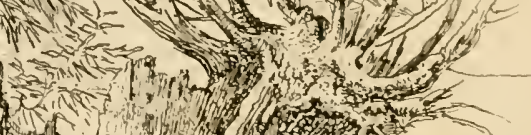

ait
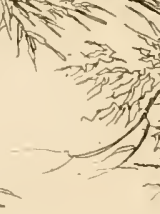

$\cos 2 x$

b)

Th

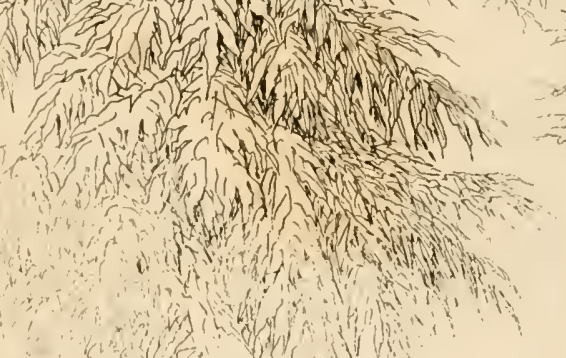

1.

1)

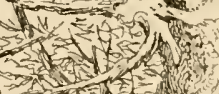

$\rightarrow=1$ in

(1)

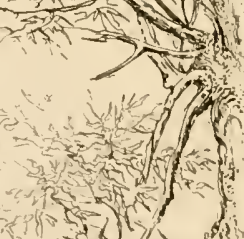

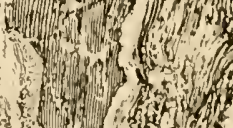

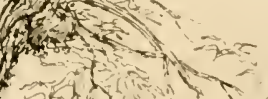

is liv

(1)
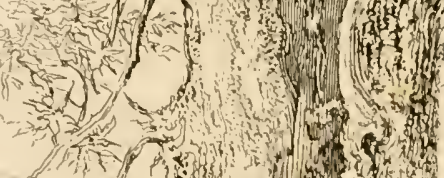

x

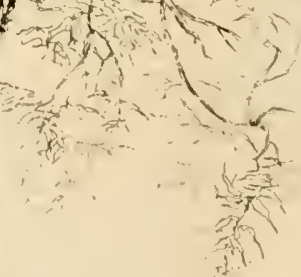




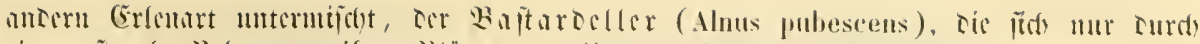

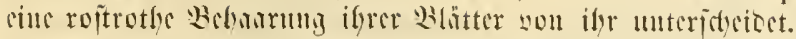

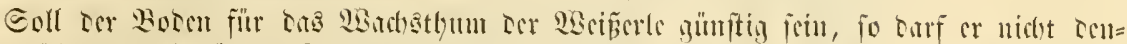

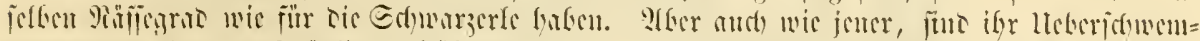

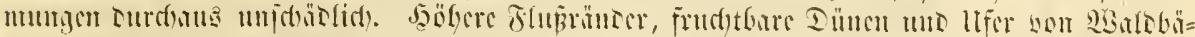

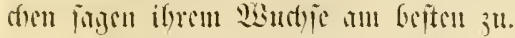

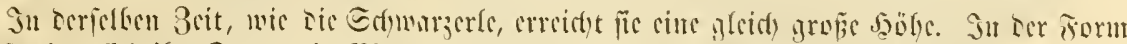

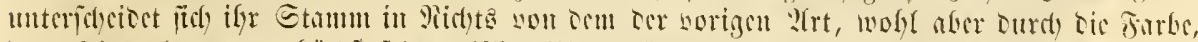

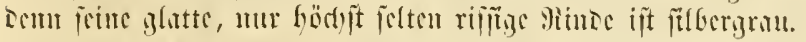

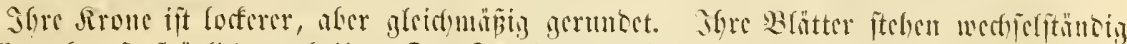

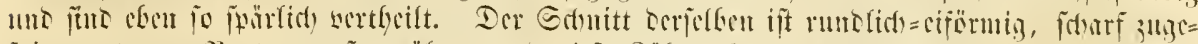

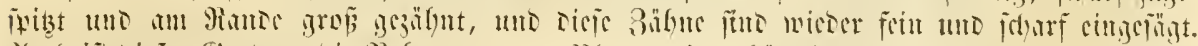

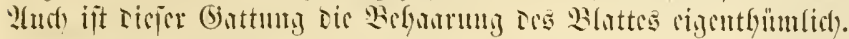

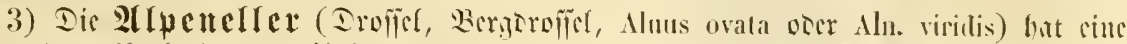

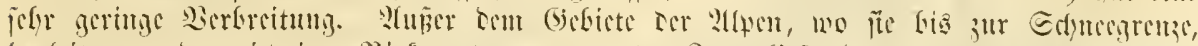

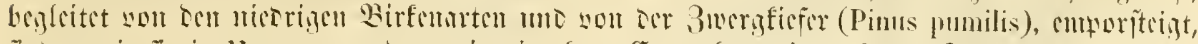

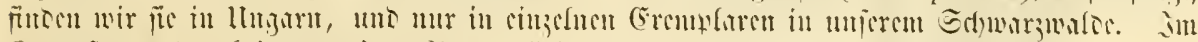

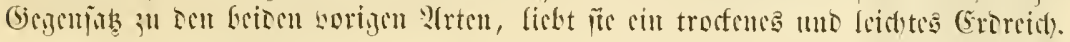

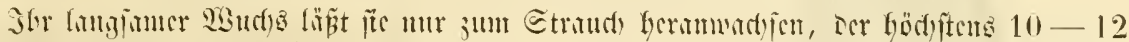

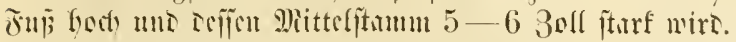

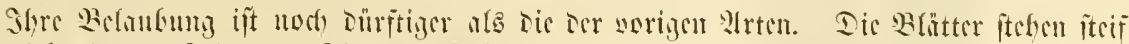

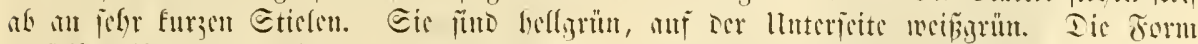

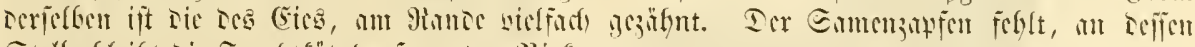

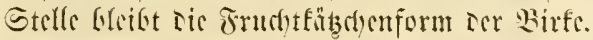

\section{(1) $\mathfrak{i} \mathfrak{L}$ II) $\mathfrak{L}$ i it $\mathfrak{k}$.}

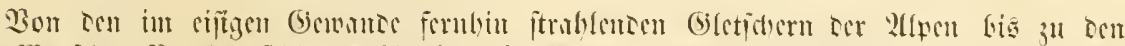

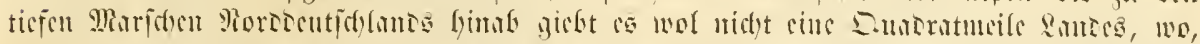

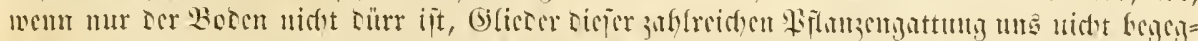

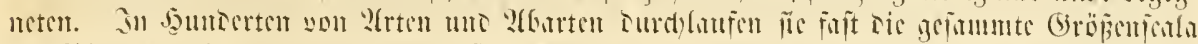

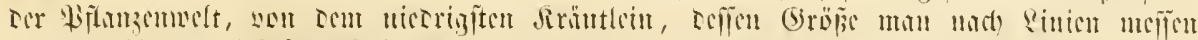

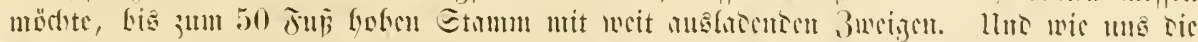

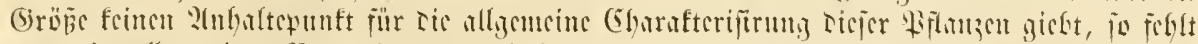

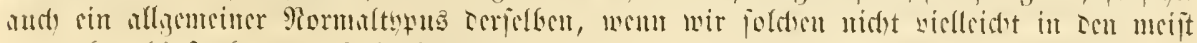

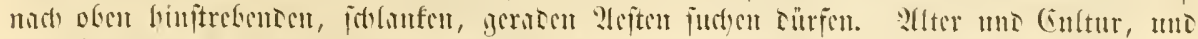

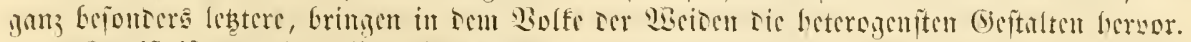

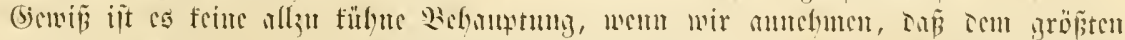

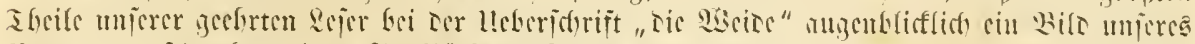

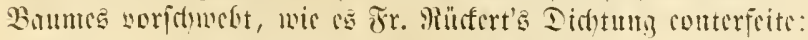

\footnotetext{
"Die 2iscice fuat foit alten Tagen

"El mundem Ettrum getruget,

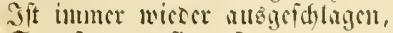

En bìt mall fic geitubet.

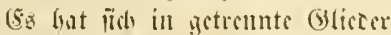

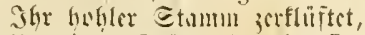

llnt icces ๔tämuschen font fich miccer

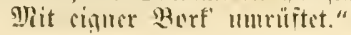




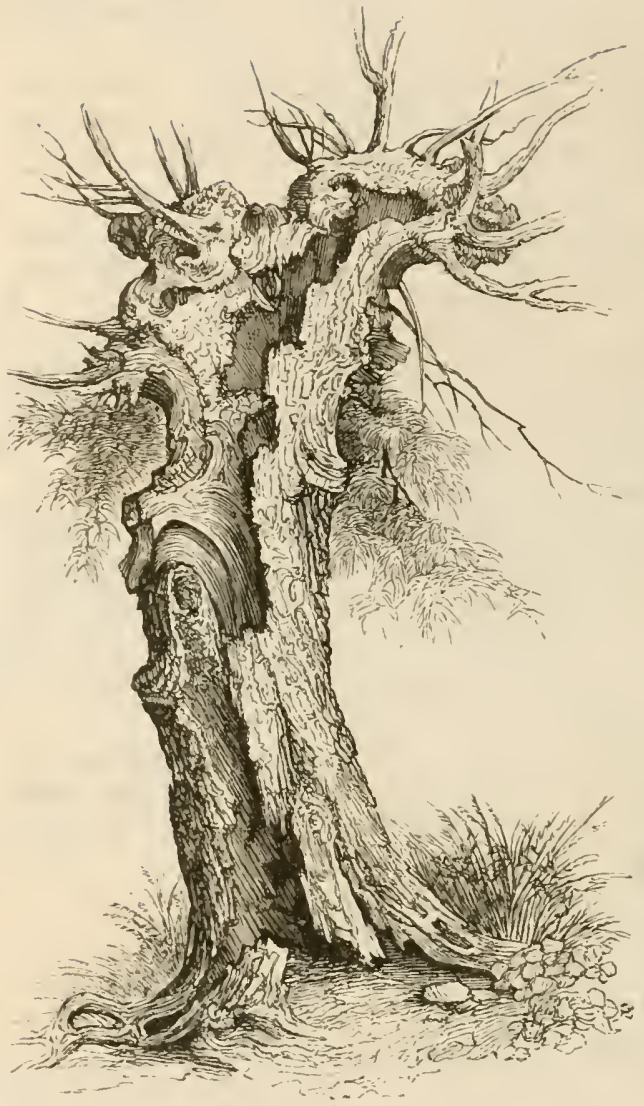

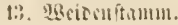

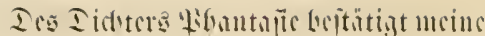

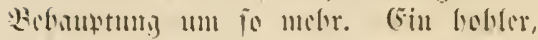

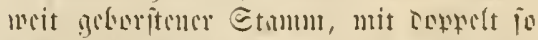

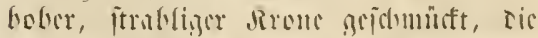

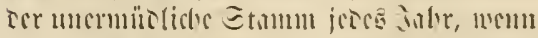

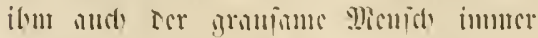
wicter tis inten solicier entreist, neu

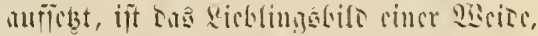

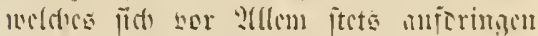

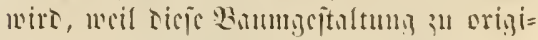

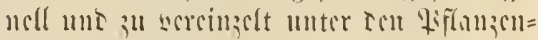

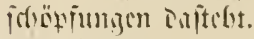

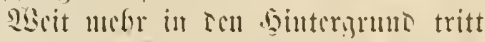

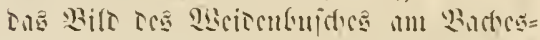

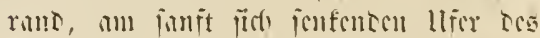
Ecrs, orer jene malerijde Jorm ter ifyri= nemerite, tis

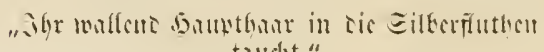
tinudit."

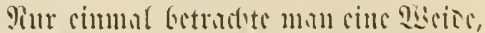

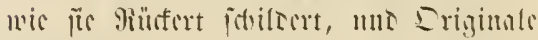

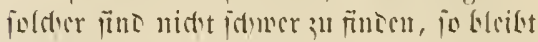

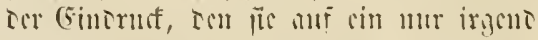

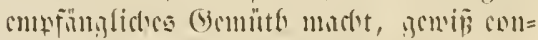

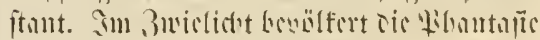

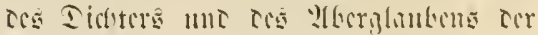

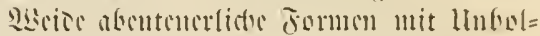

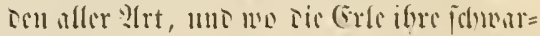

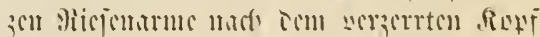

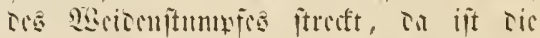

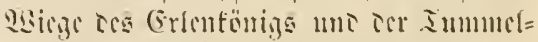

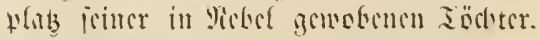

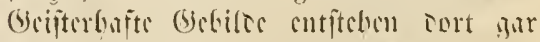

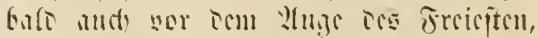

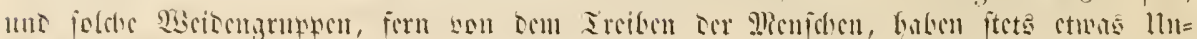
beimlicters.

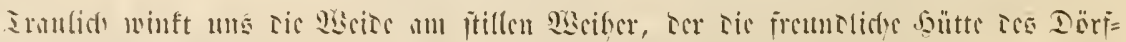

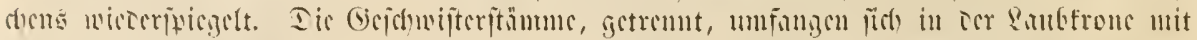

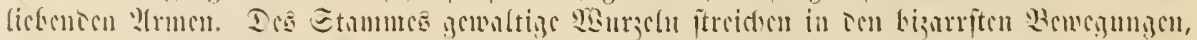

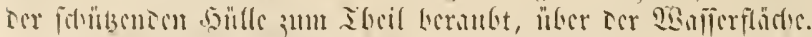

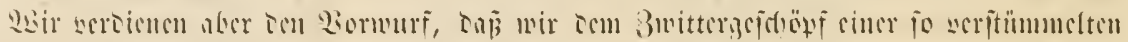

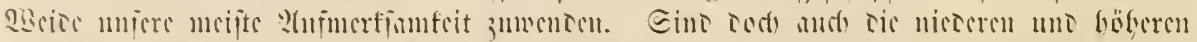

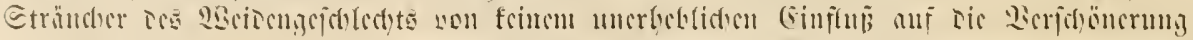

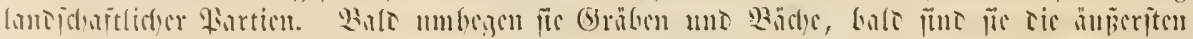

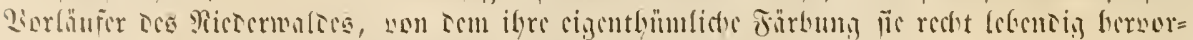

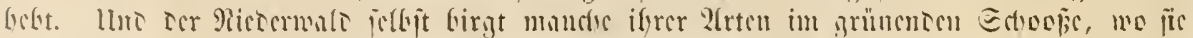

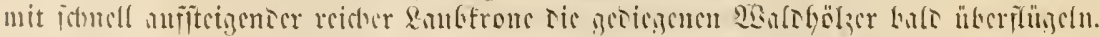

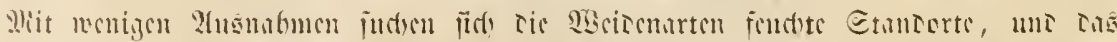

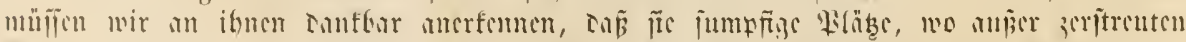

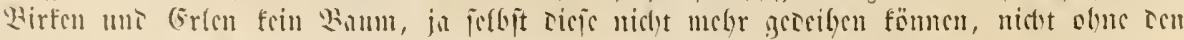

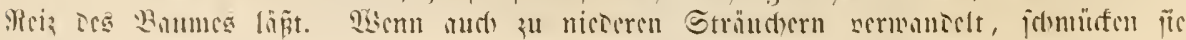




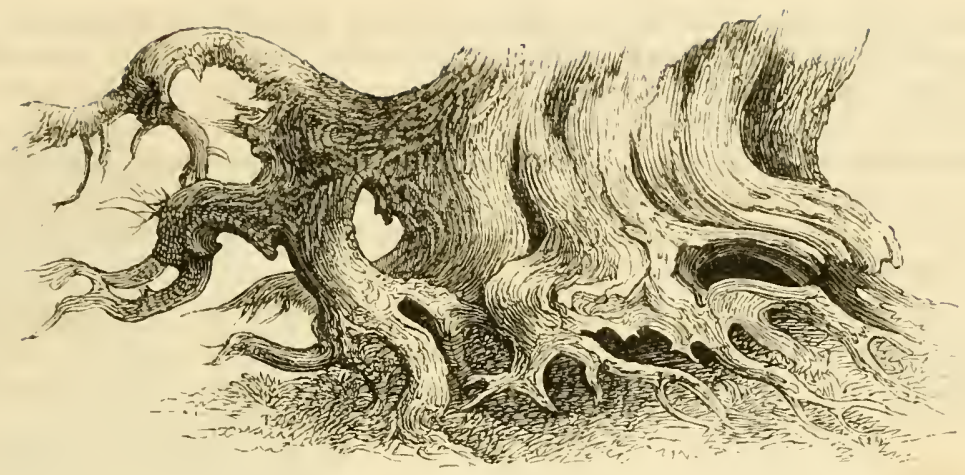

44. TSurjel or Mgcise.

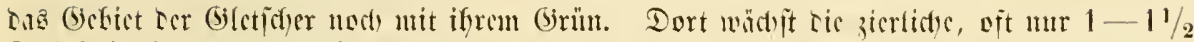

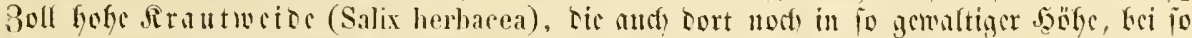

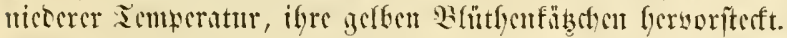

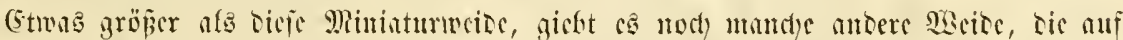
ธen Göd)

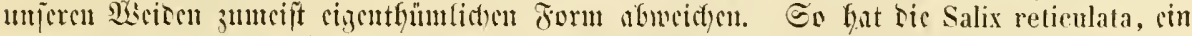

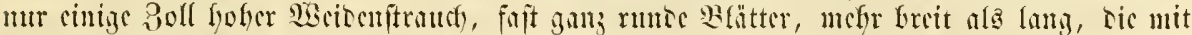

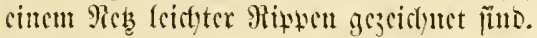

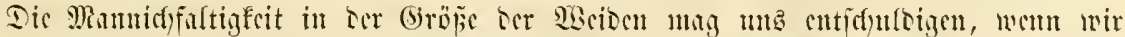

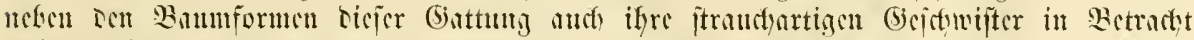

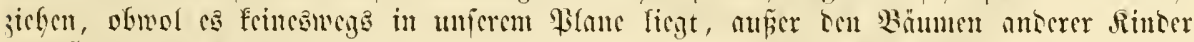
iç Madocs zu yctenfen.

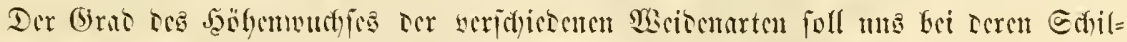

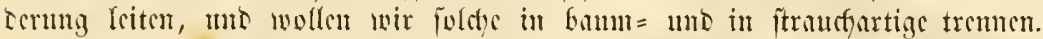

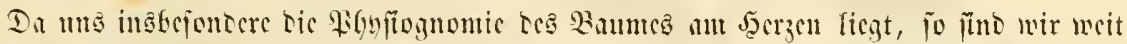

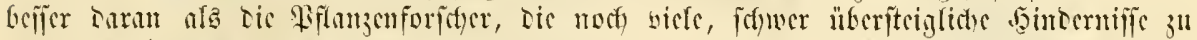

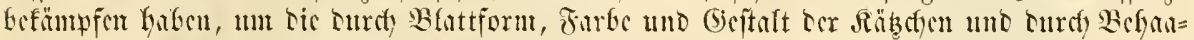

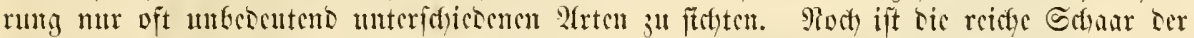

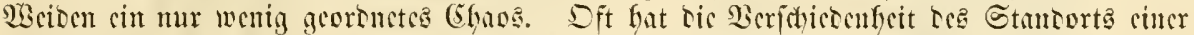

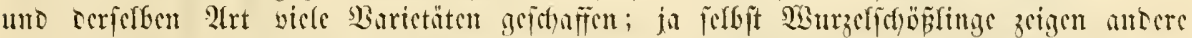

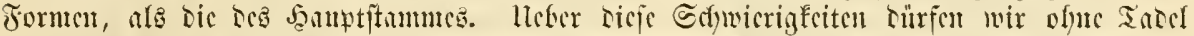

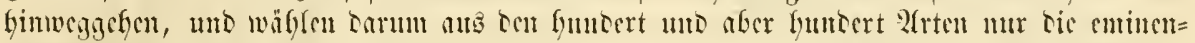

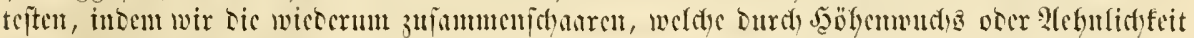

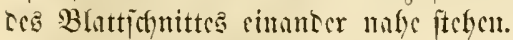

3on ocu baumartigen Misciocu ijt:

1) Dic Sobl = ofer Saalweibe (Salix caprea) tic bofmutcite, wril fic bic bei

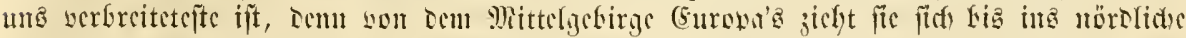

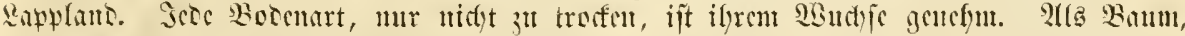

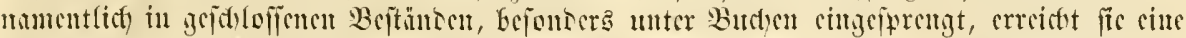

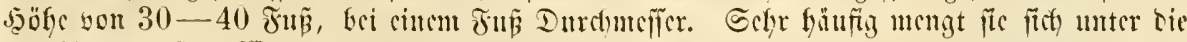

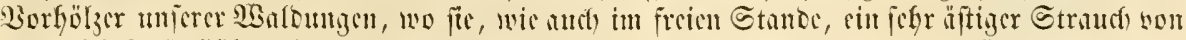

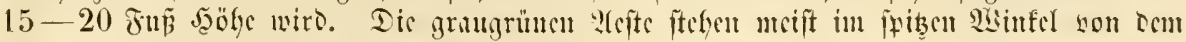

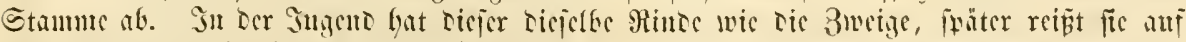

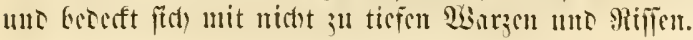




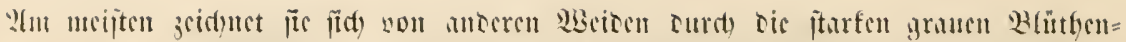
tübs)

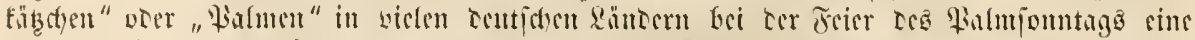

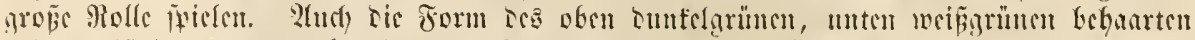

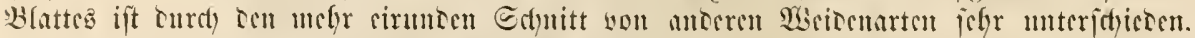

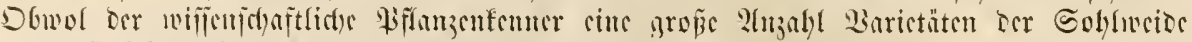

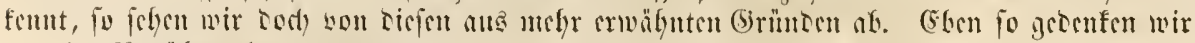
nur int Boribergelyen

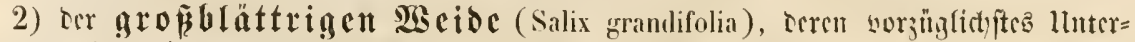

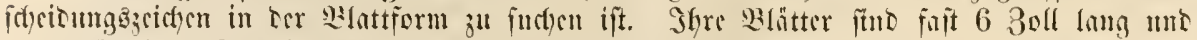

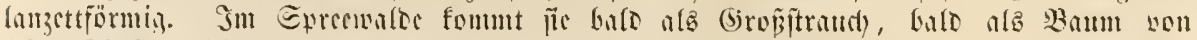

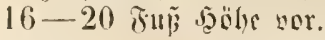

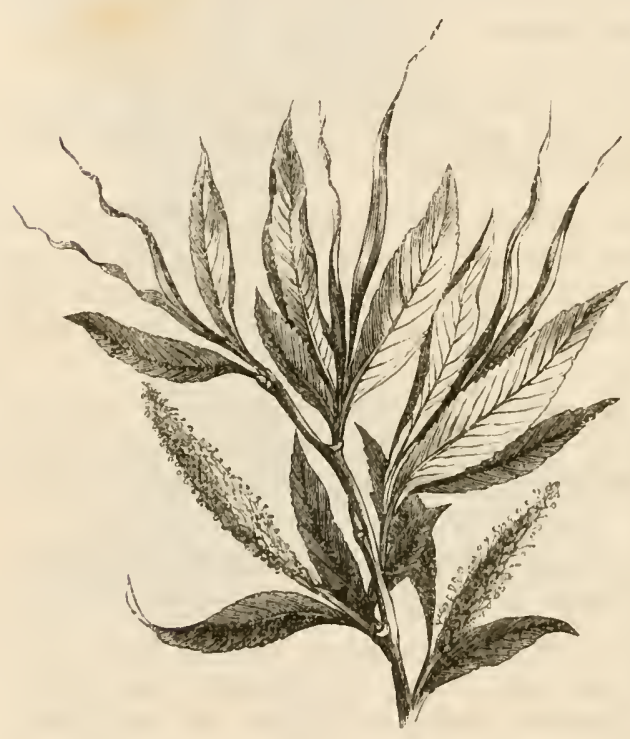

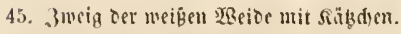

Durd) Sic Blattform, mem and) in

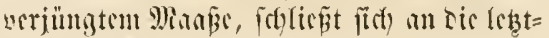
genamute ?(rt an:

3) Dic wcipa 2 seide (Bam=

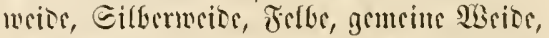
(Scrberweitor, Salix alba, Limn.). Sic ift sine in fait gunt Europa gemeine Bamt=

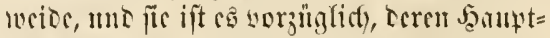

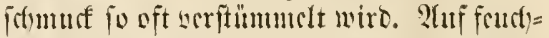
tem Boten, fowel in Ricocrungen als in Borbölacrn, errectet fie jefurll ibren büd)= ften 23 แt Durdmenfer. Dodt follen an tom llfer tra

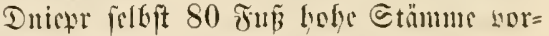
fommen. Durd) Das Röpfen ier 2(cepte hürt

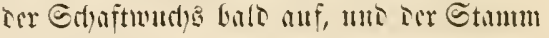
(mingt firt) in rincumuntors= (id) uno man= nict) find gi:= ftalteten

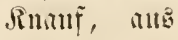
Dem Dom tis nemen id)lan=

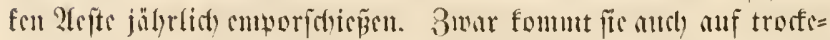

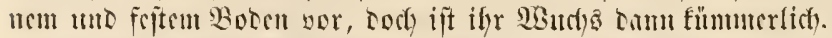

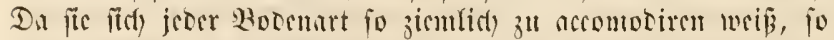

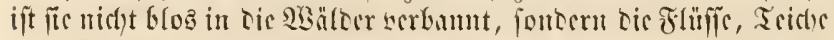
uno Wisfen futto oft nou ilys bejergt.

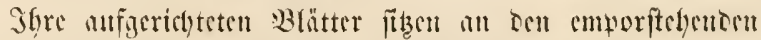

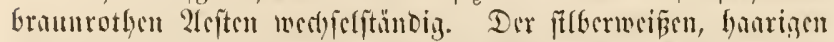

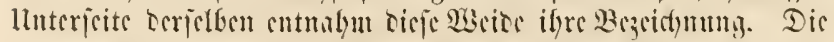

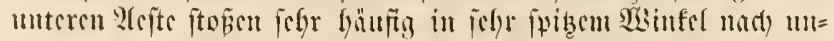

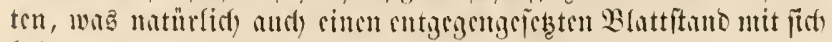
brimigt.

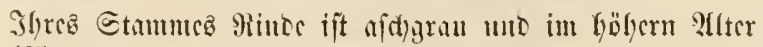
feftr rifitio.

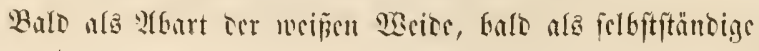
2(rt nennt mtan

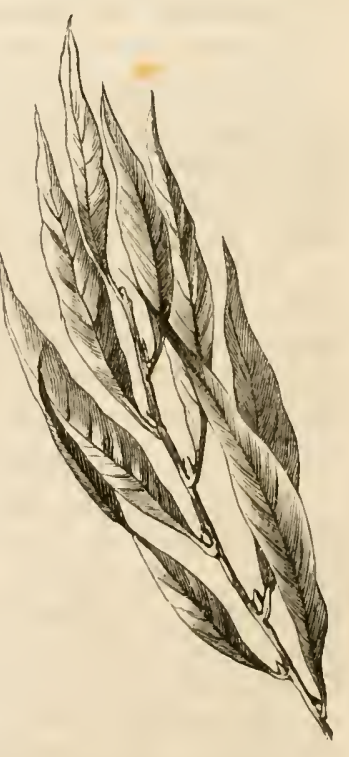

46. Salutituns rer unteren ?lefte vou

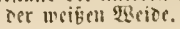




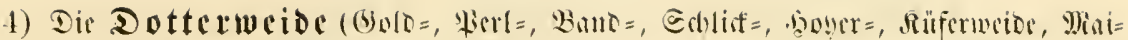

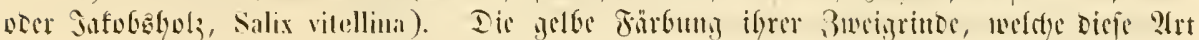

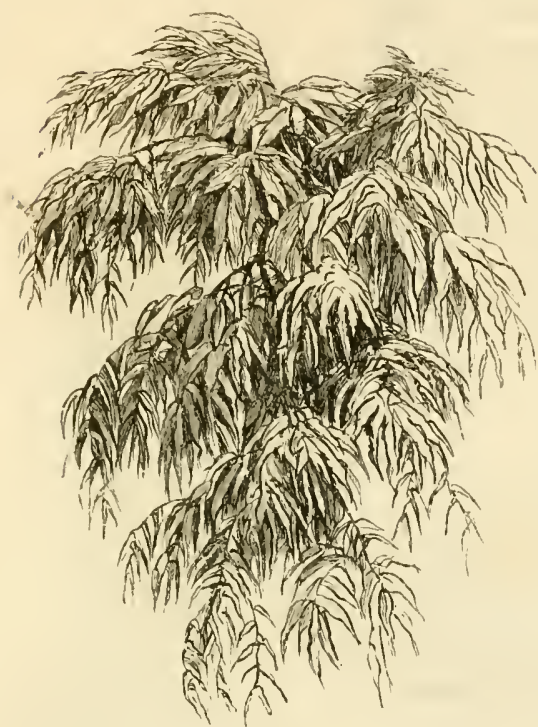

17. Eanbgrmye Der Dotterwecios befontors in 23 inter marfirt, unterjacioet fie sou

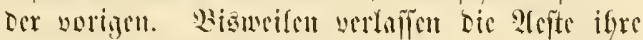
normali Midstumb tuto netigen fiet nach untert.

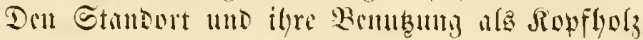
bat fie mit dor yorigen gemeits.

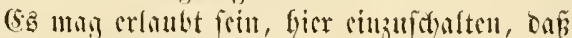

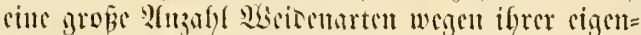

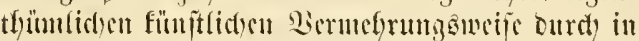

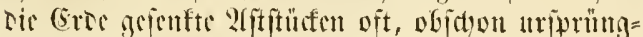
(id) Bamu, and Lic Etraudborm munimmt, Deren

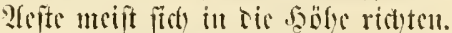

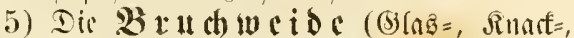
Expöbefuecioe, Salix fragilis) itteft Den sorigen

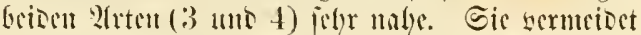
meijt jumpingen Bosen, liebt aber Fflús= uno

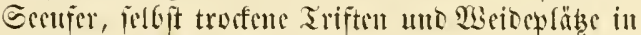
falt allen Eänoern (Guropis.

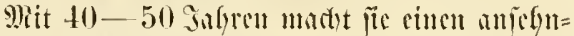
lid)

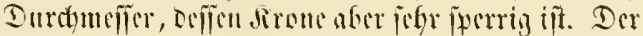

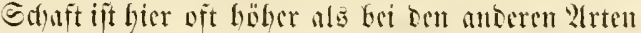
mo itarf rifitis. Die Rinos oer 3 weige ift braun=

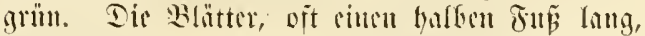

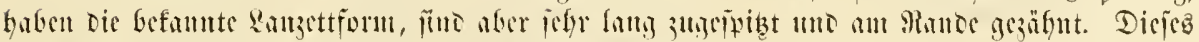

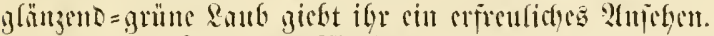

6) Dic braune 2beide (Salix Russeliana) bat jum Ituteriotico non ter Bruttuctoe

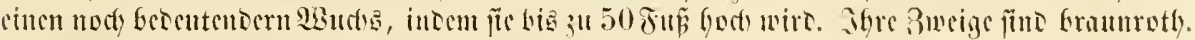

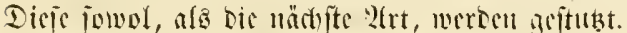

7) Dic breitbrattrige 28 cide (s.

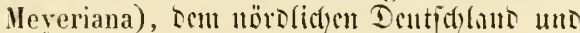

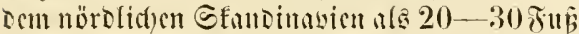
Gober Baun rigenthüm(ict), bat lanzettiör= mige Blätter, bie alsagenad) 6 3oll lami

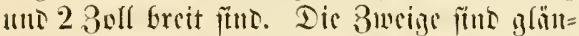
zenterinut.

8) Dic Sorbecrucide (Salix pentandra), cinc in gunz (5uropo, iclbjt int boben Porben uno auf ziemlich lantyen Strofen ze=

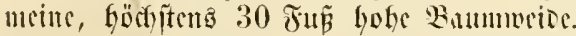

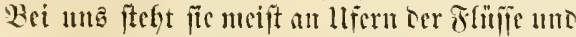
in Felogölzern. Dicje jet)öne Biscicenart ijt fait mit jeom Boom zufricom, folto ala Etrauta), buld ala baum.

Shre Bläter funo ciförmity 1110 feill ; gejpikt. Dit mattgrüne llnterieite serielben ijt nicht befourt, wie aud bei ben woritelyen= oen 2 frtitu.

9) Dic Fräbrocioc (Salix praecox). cin Icm Güch Dentid)lants angeförizer

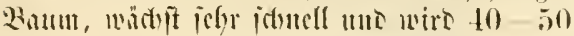

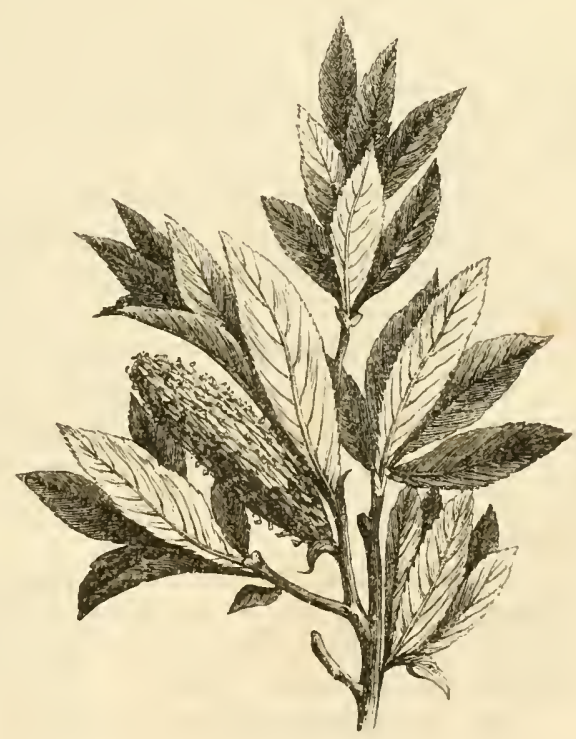

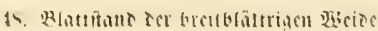
$f^{*}$ 


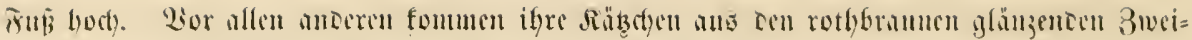
gen lerwor. Finzufer fint ily Standort.

10) Iir sratter= ooer Şängeweide (Silix babilu-

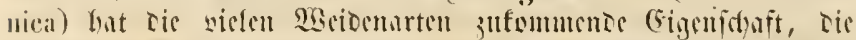

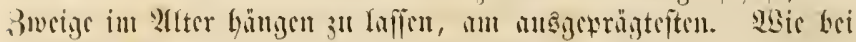

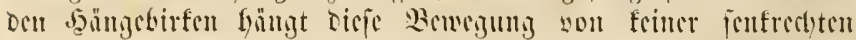

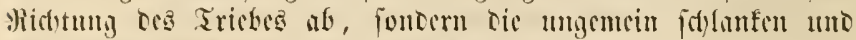
iurten Bucige meigen fïd) ourd) cigene E(t)were nath unten. Rod)

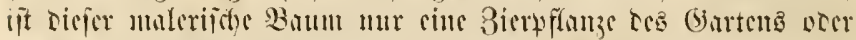

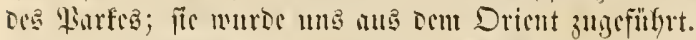

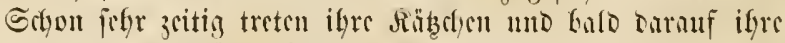

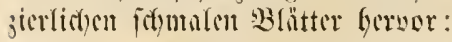

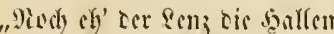
Dẻ Sarineó frich belant,

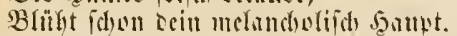
Du biit
QIn peine Écnoming glunbt." (Ěrl Berf.)

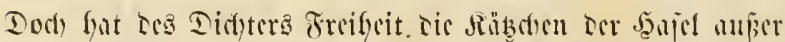

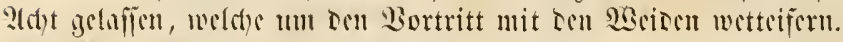

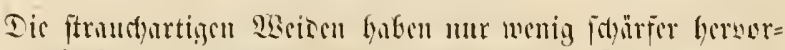

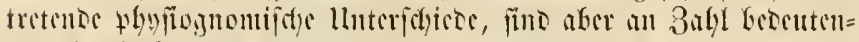
Der ala dic Gmmartigen.

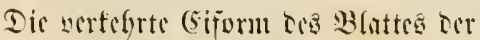

11) Ealbeiweide (Salix anita) betsu wir berwor, wril

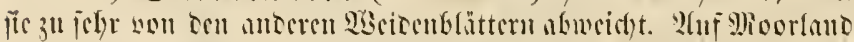

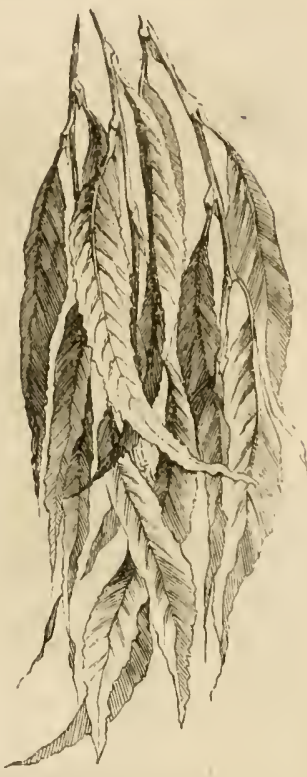

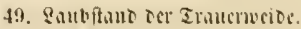

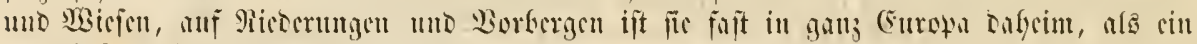

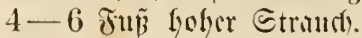

12) Dic Sanrució (Salix aquatica voer Salix cinerea, Limn.), nicht filten an

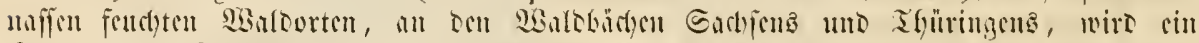

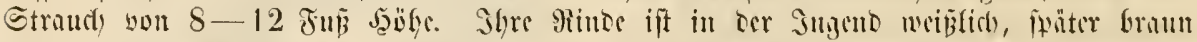

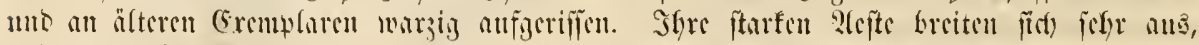

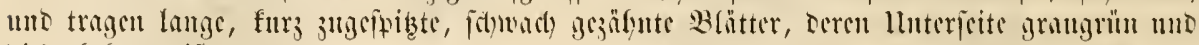
Didit befourt ift.

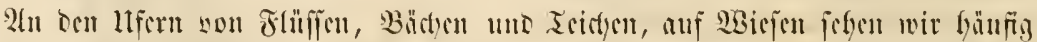

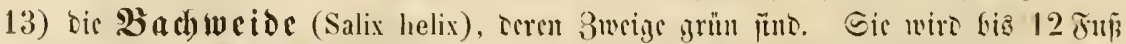

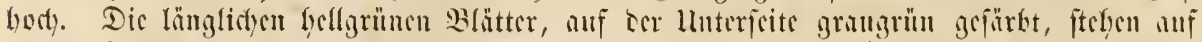
furgen @ticlen cimnober gejenülor.

14) Dic Mothucide (Salix mubra) mo

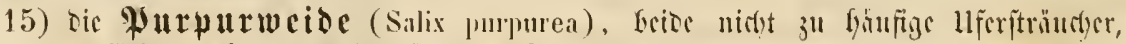

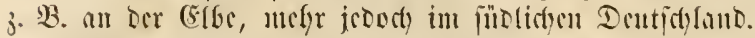

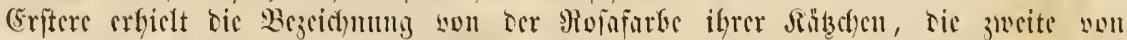

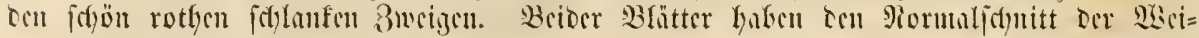
Denblüter.

16) Dic Rorbweibe (Salix viminalis), mit ferr fomalm, 4-6 3oll langen

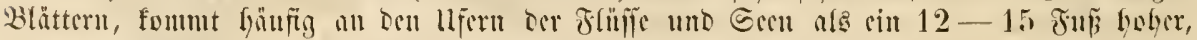

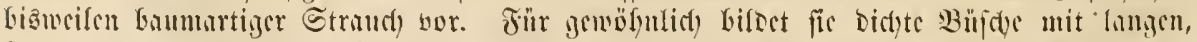

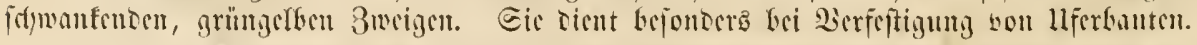

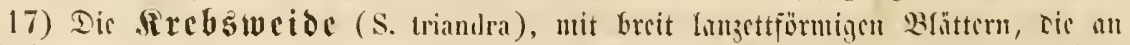

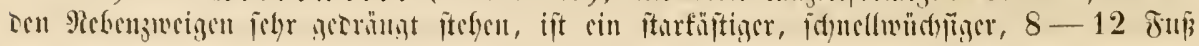






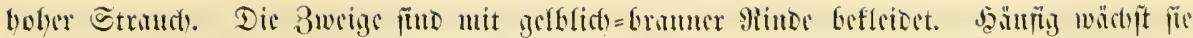

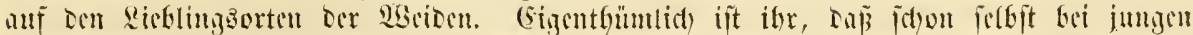

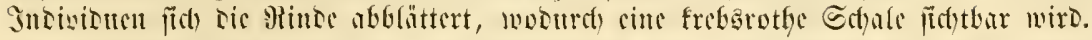

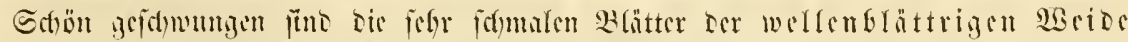

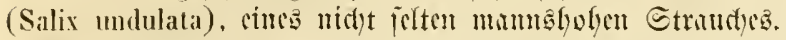

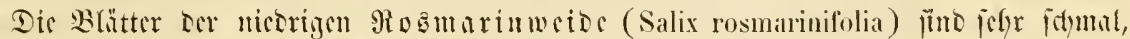

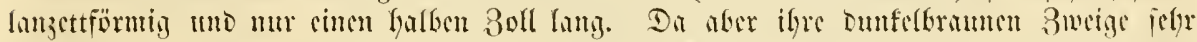

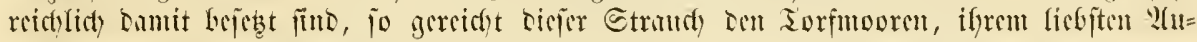
fenthalte, jefyr jur Bicroc.

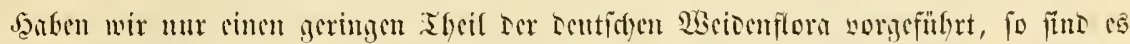

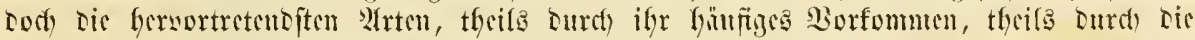

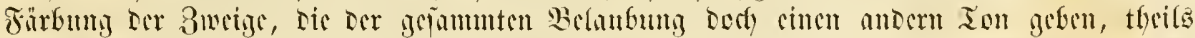

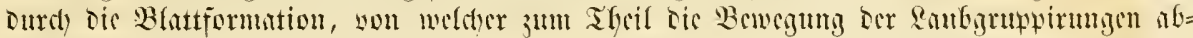
băningut.

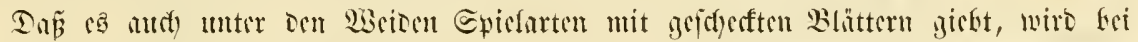

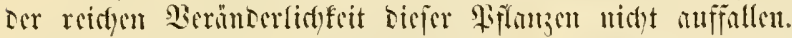

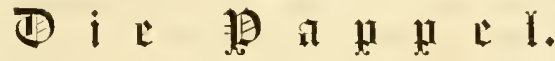

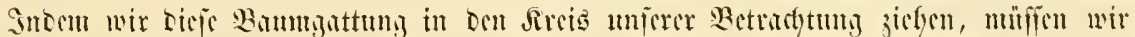

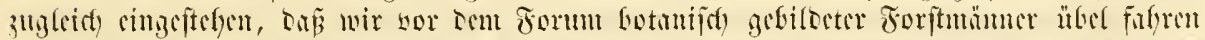

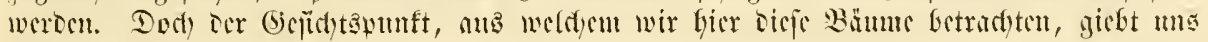
volles Micift torju.

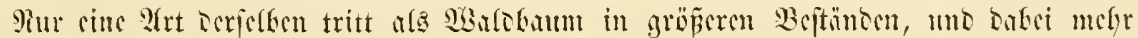

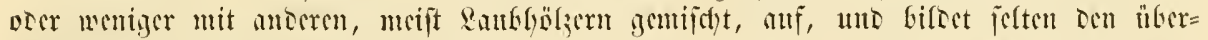

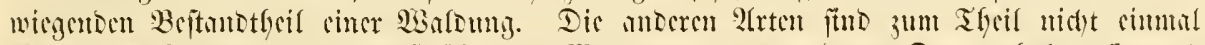

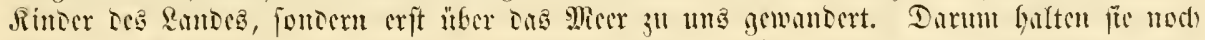

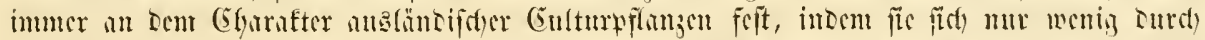

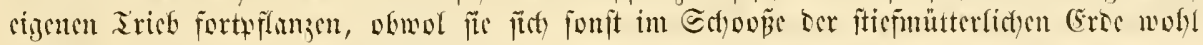
ju fülylen jót)cineu.

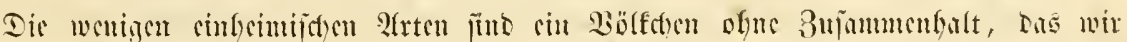

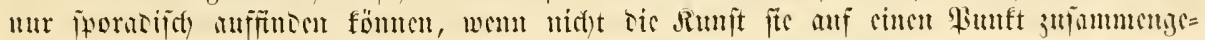
fiilyert biat.

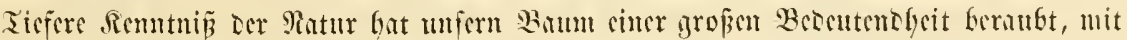

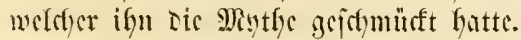

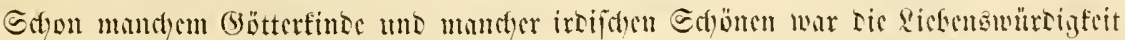

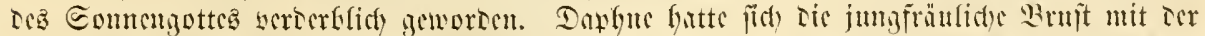

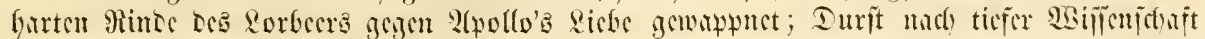

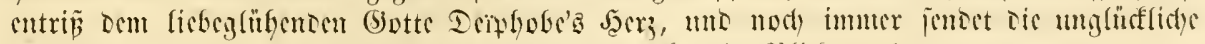

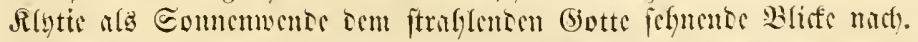

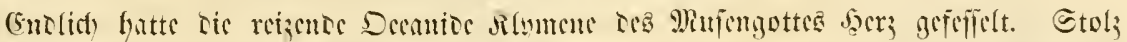

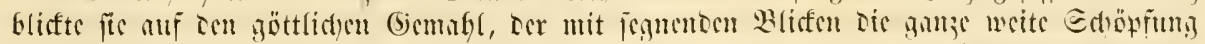

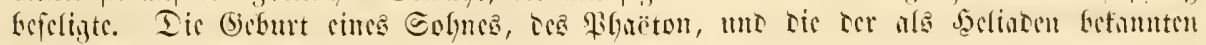




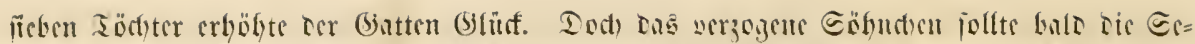

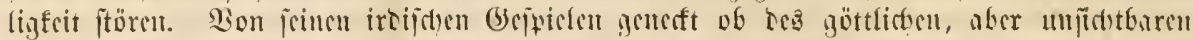

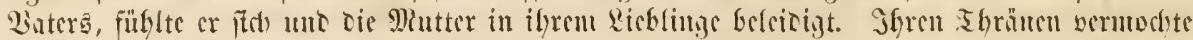

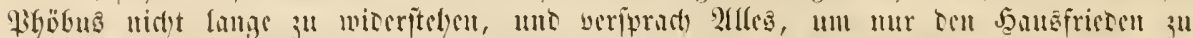

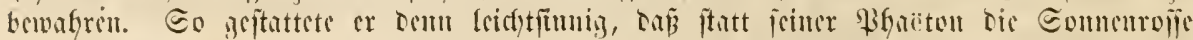

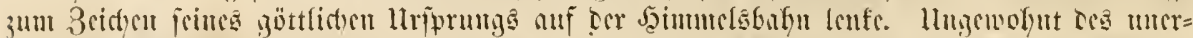

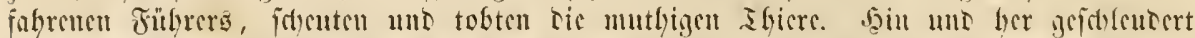

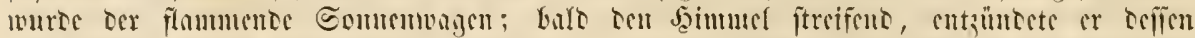

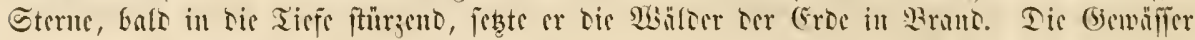

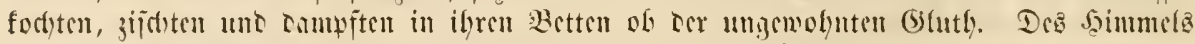

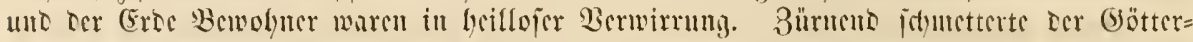

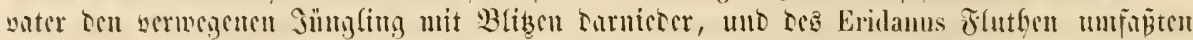

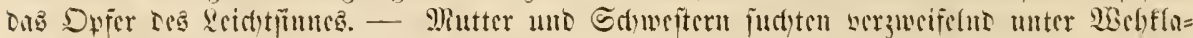

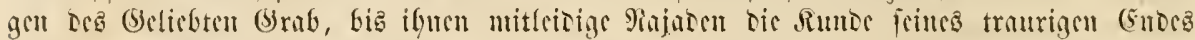

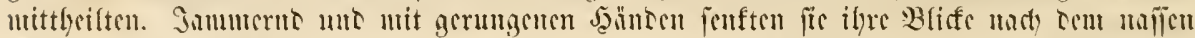

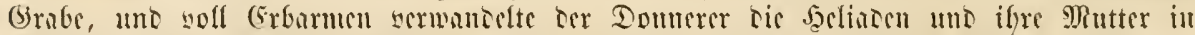
Waptelu. Shre Thränen erbïrteten 34 Bernitein.

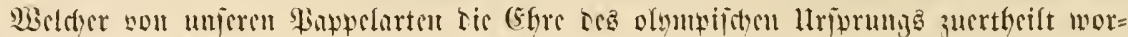

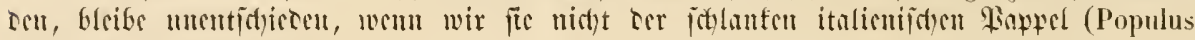

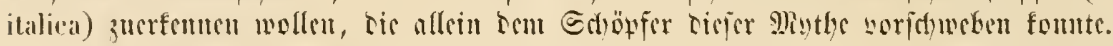

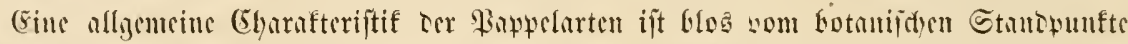

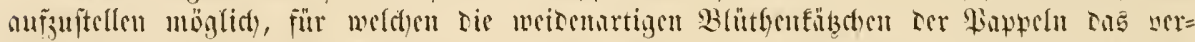

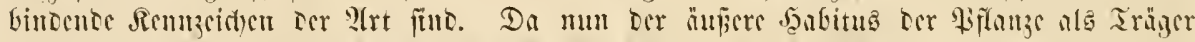

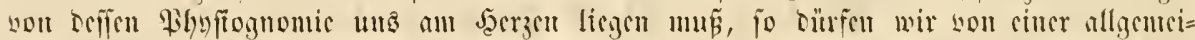

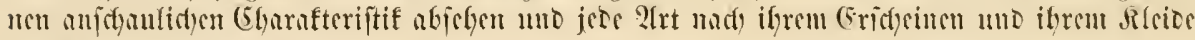
ing ?) ?uge faाfen.

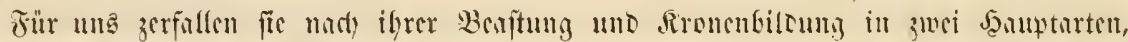

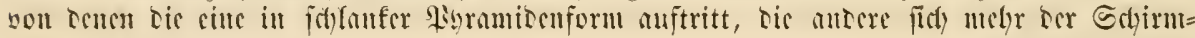
form näbert.

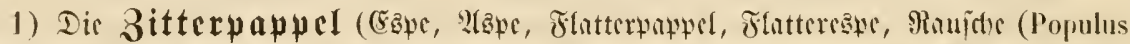

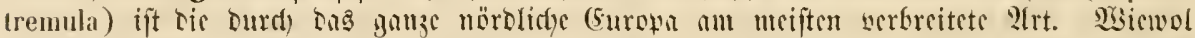
fie in Borfölzern, in fleincren Bbaltwarticn, folfịt in gröferen Forften anftritt, jefören

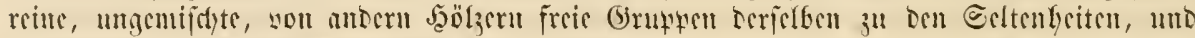

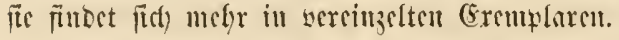

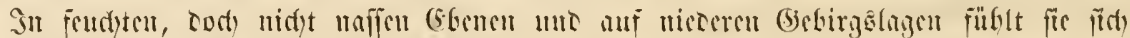

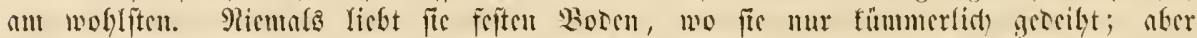

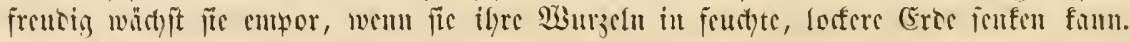

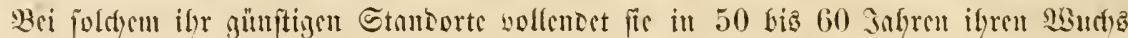

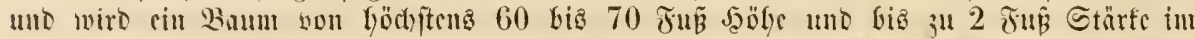

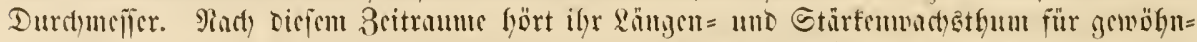

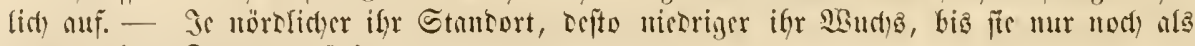
juerigartiger Etratud auftritt.

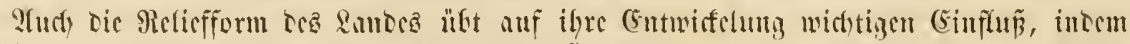

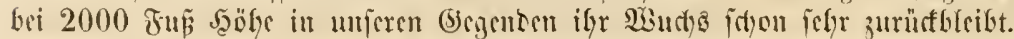

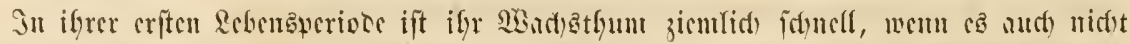

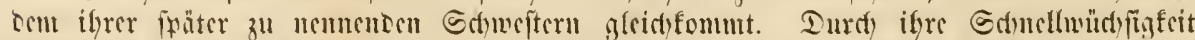

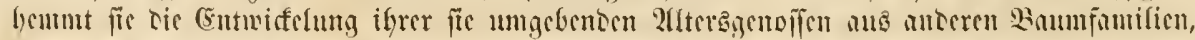

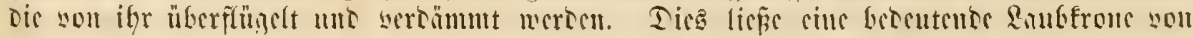




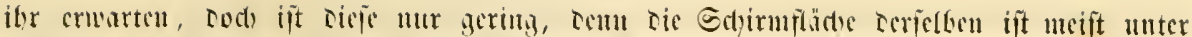

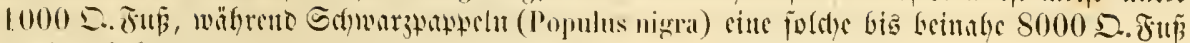
arreidet baben.

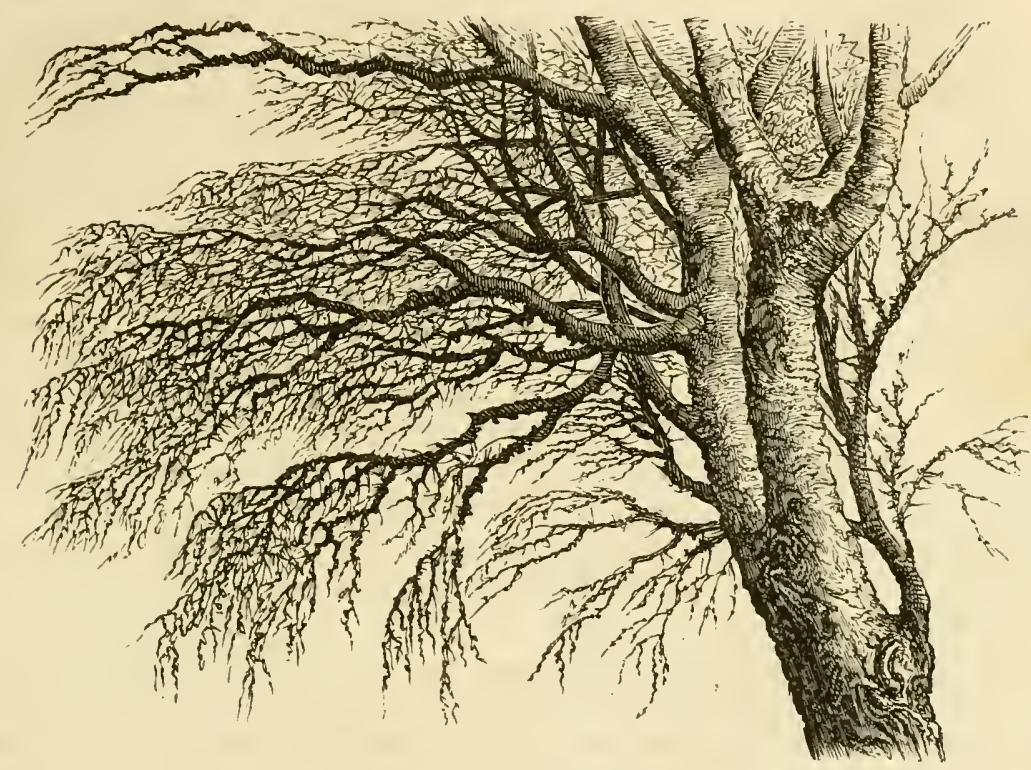

5). ?titbat Der 3itternawet.

Der Walgenförmige Gtanm ift bei älteren Bäumen mit ciner jowärzlichen Rinte be=

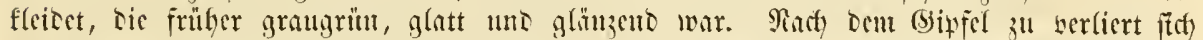

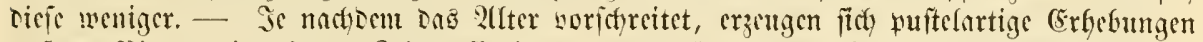

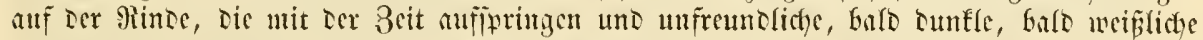

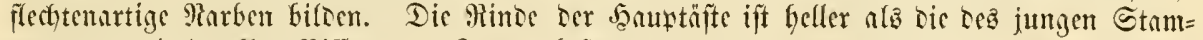
แte uno mit tunflen Riffen uno Rnoten bejeçt.

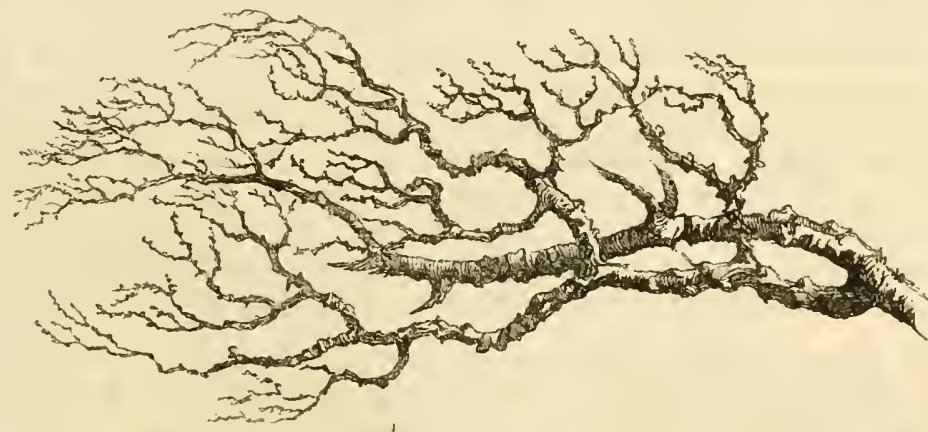

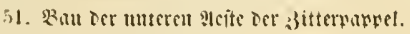

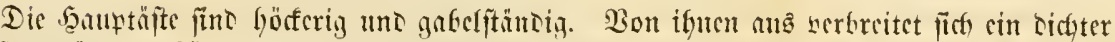

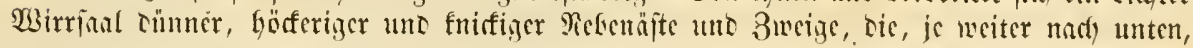




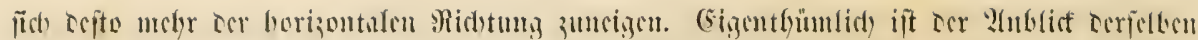

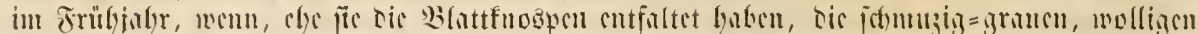

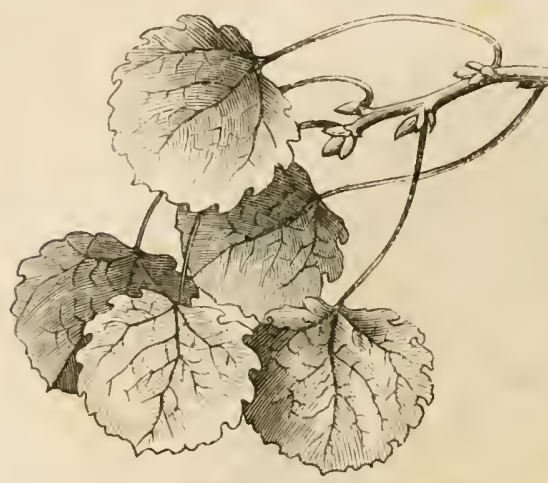

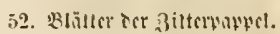

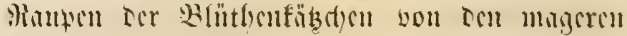
?hiten berabbümon. Eelfit im Eommer tritt

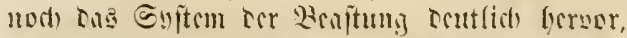

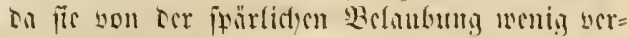

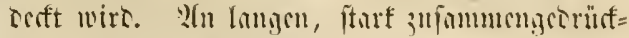
ten Eticlen arjittern ionon bri som leifiten

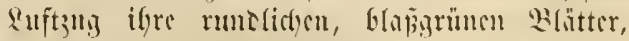

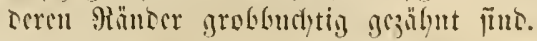

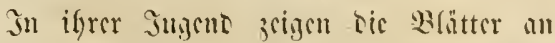

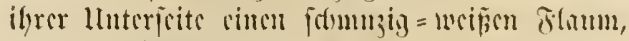

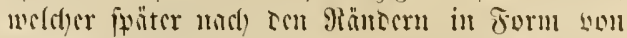

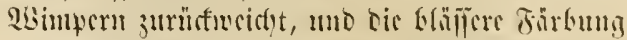

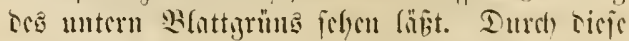

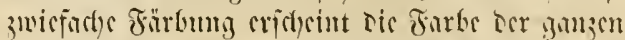

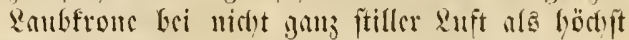
unbeitüntig.

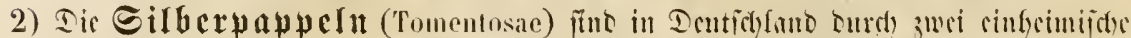

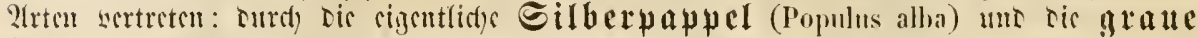
Tlappel (Populus canescens).

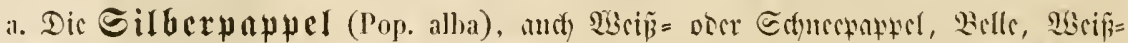

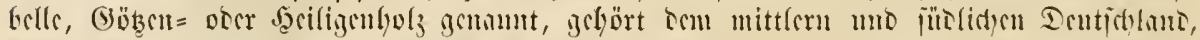

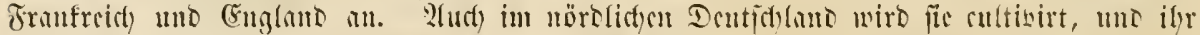

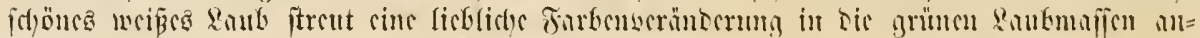

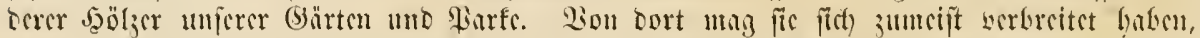

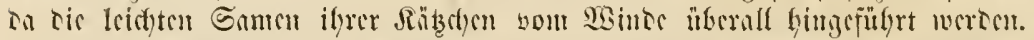

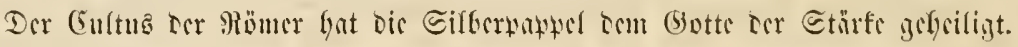

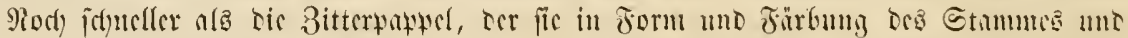

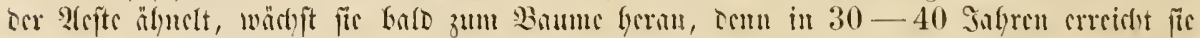

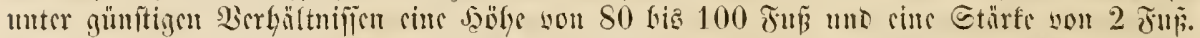

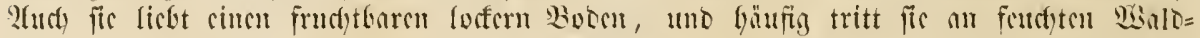

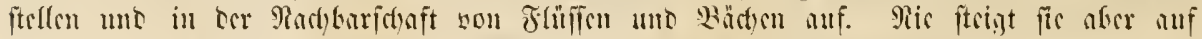

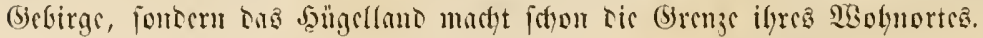

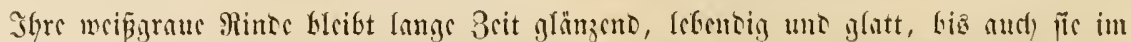

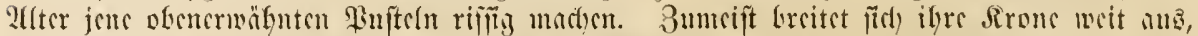

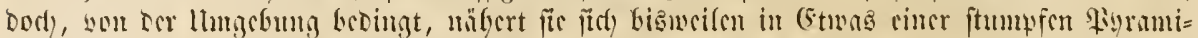

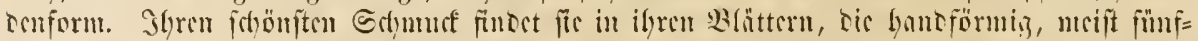

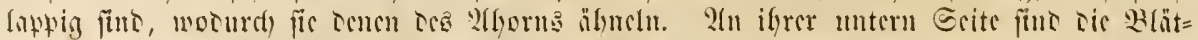

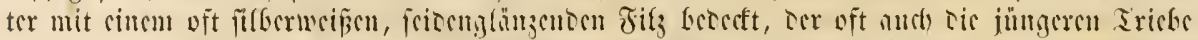

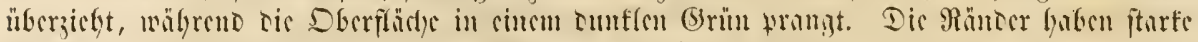

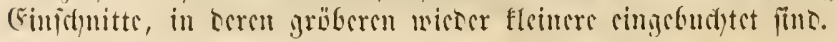

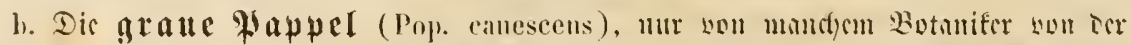

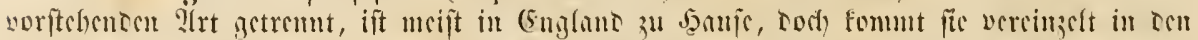

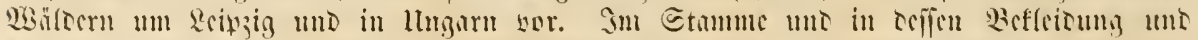

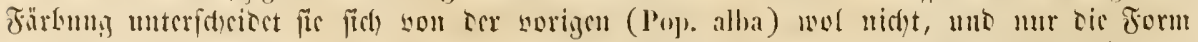

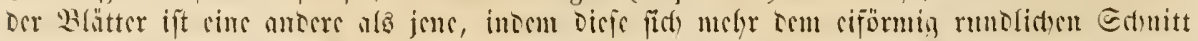

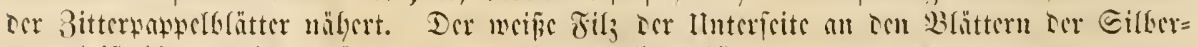

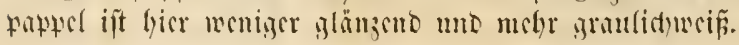




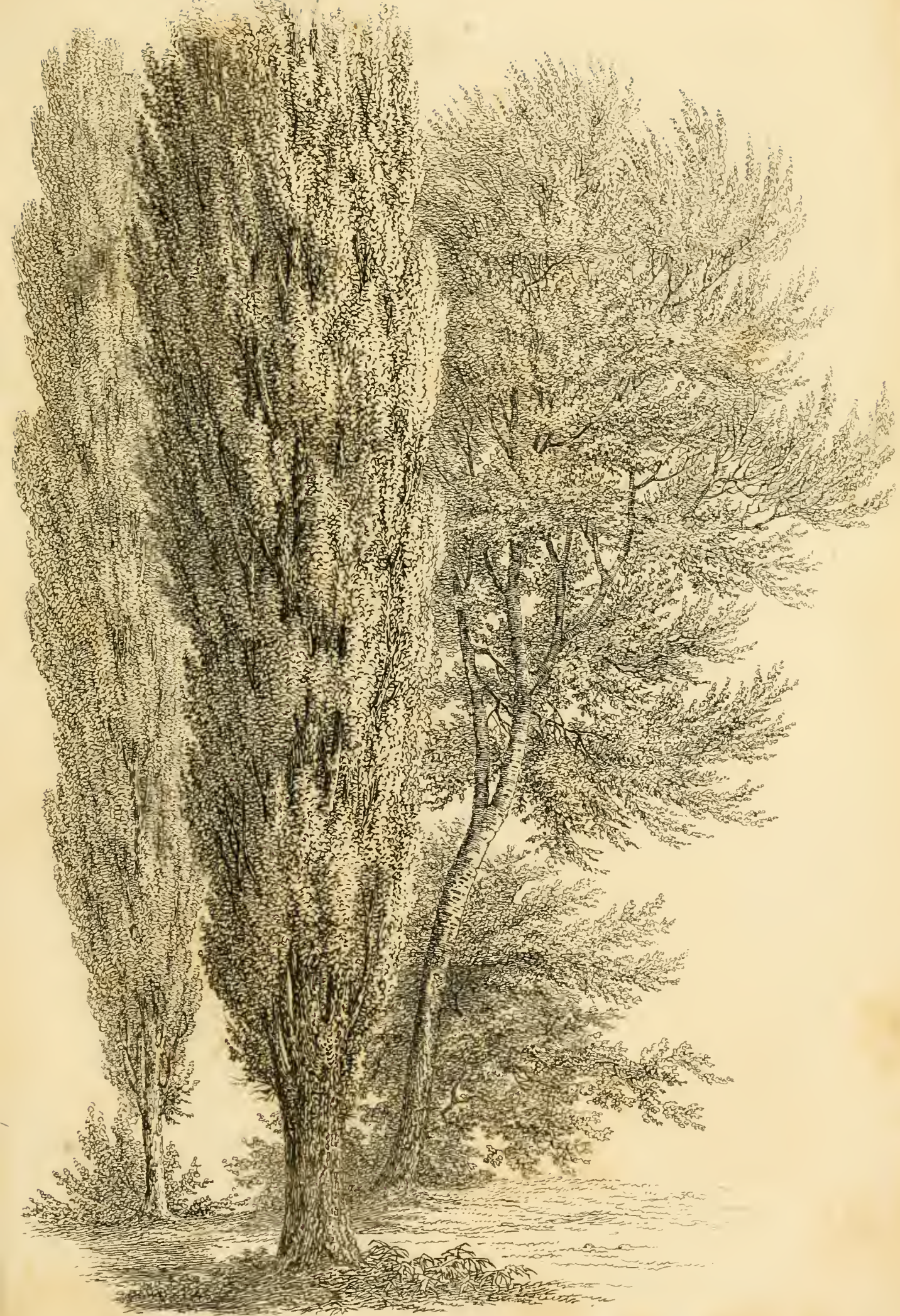

Die 3itterpapper uno italieniscte Tapper. 



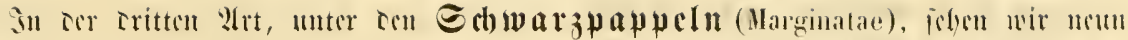

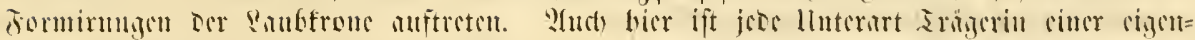

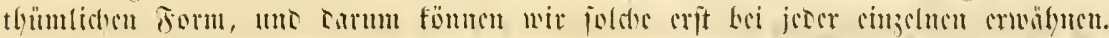

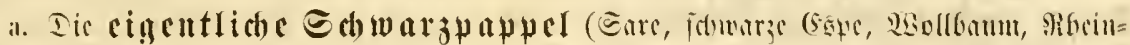

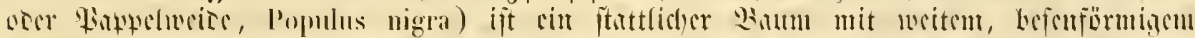

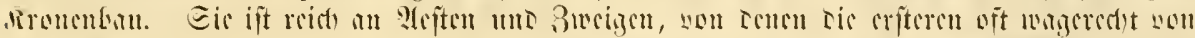

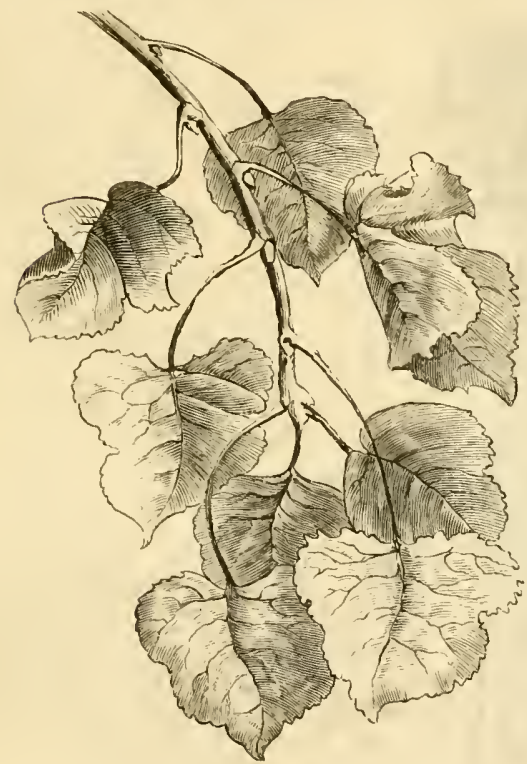

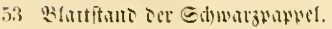

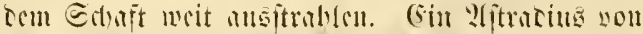

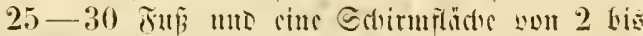

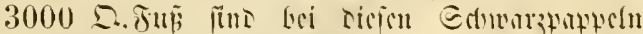

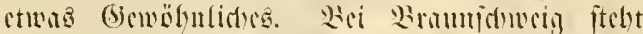

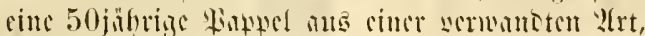

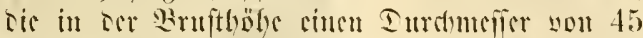

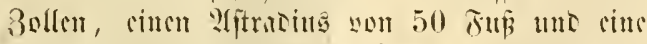

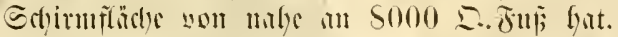

Durd) Das ?llter crlatert and iber Etmmm=

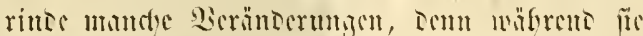

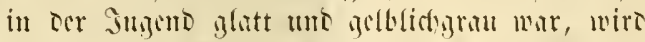

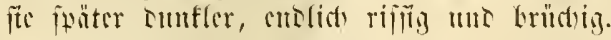

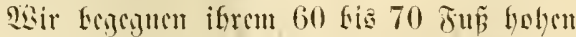

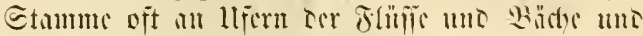

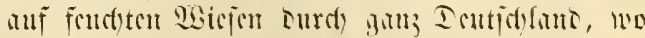

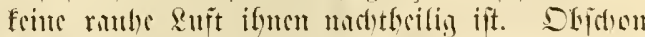

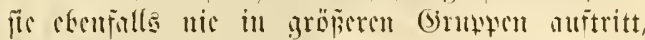

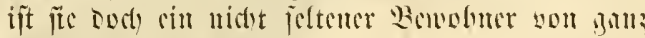
(surotis.

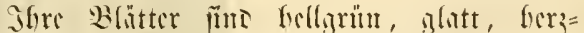

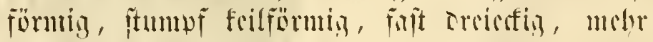

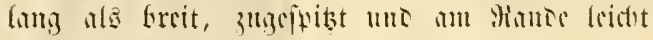
greingt.

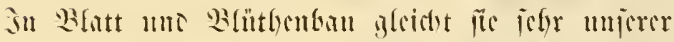

1. geneinen orer italienifden Pappel (Pop.

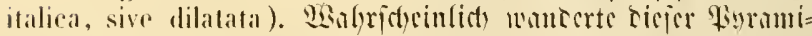

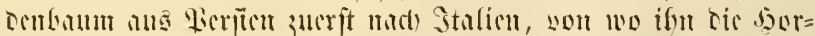

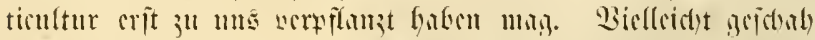

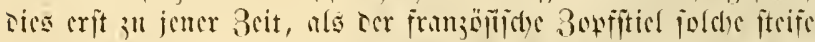

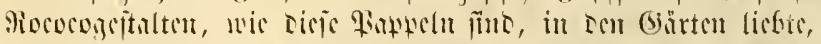

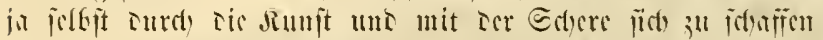

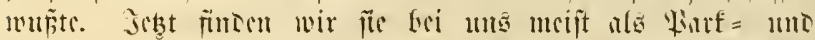

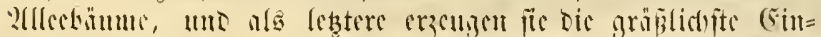

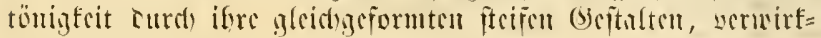

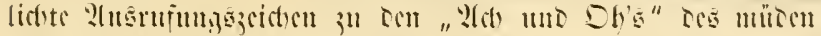

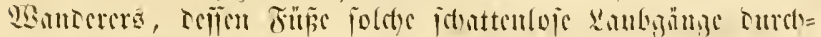

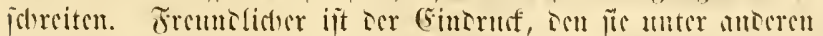

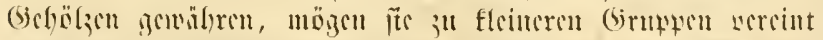
vocr ijolirt jefoen.

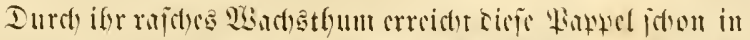

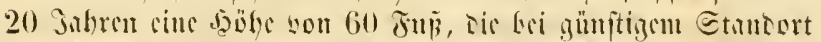

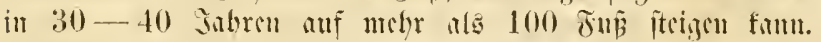

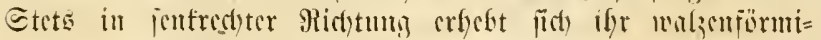
zer Stumut. Sou sube bis zum bipipel ift or mit ticj

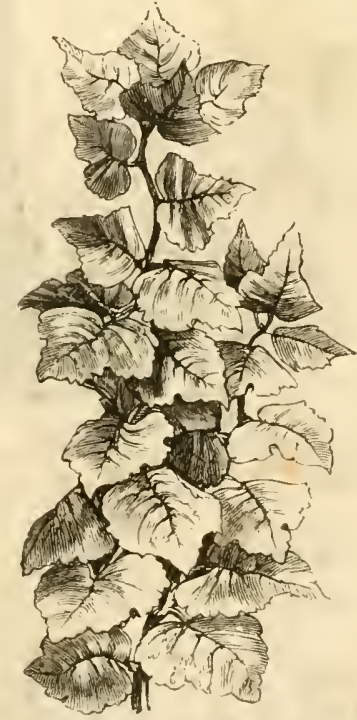

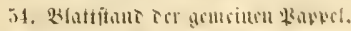




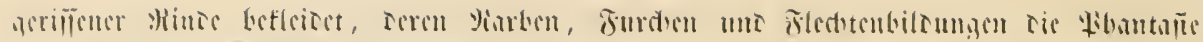

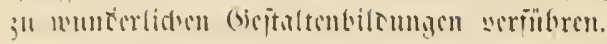

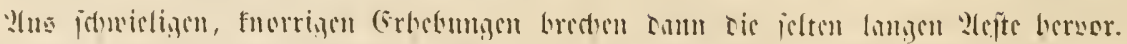

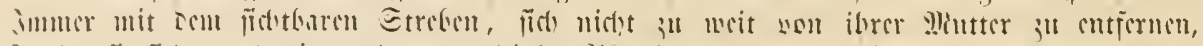

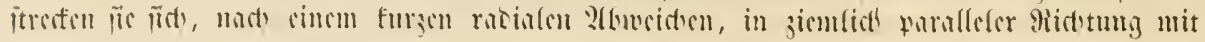

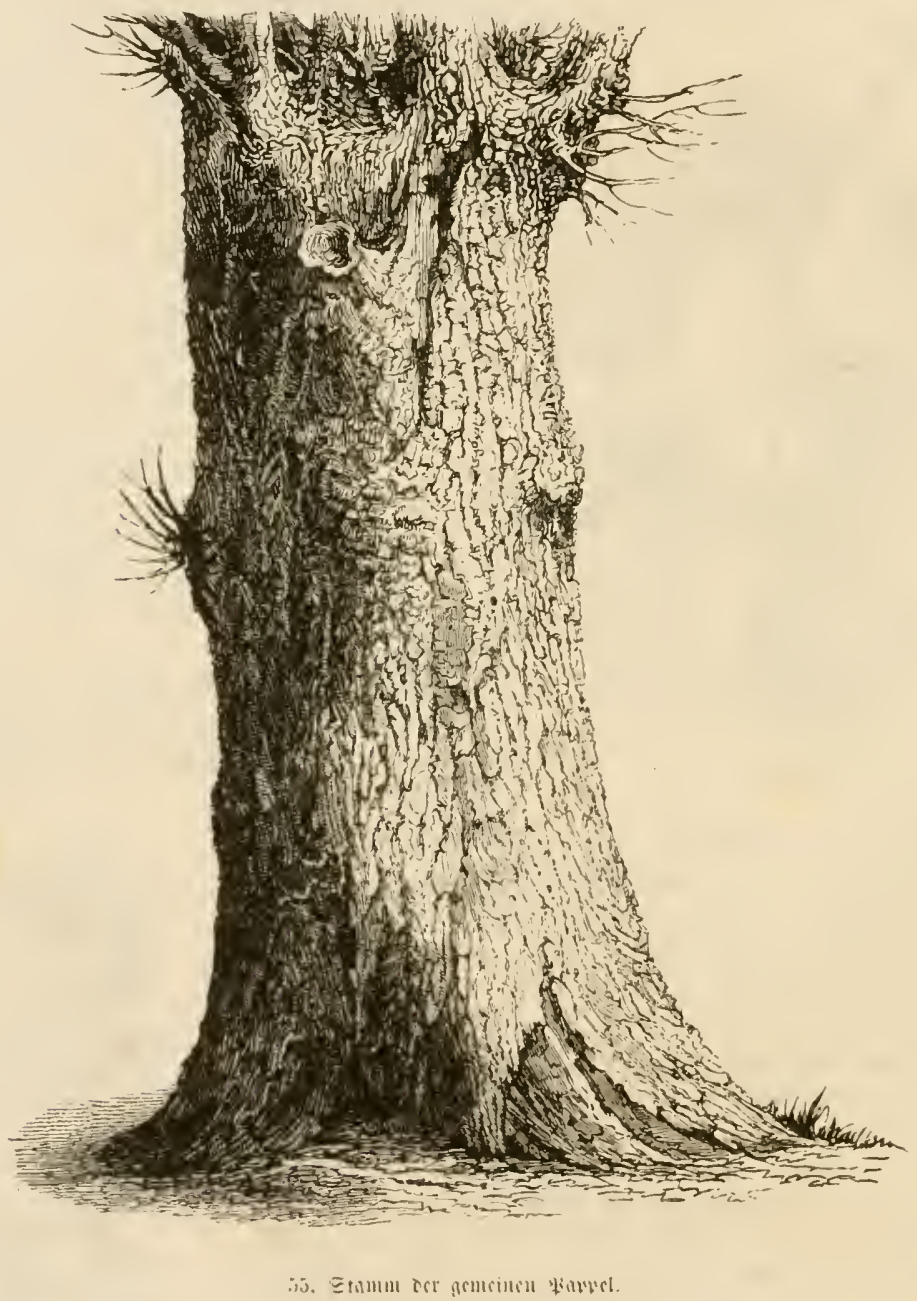

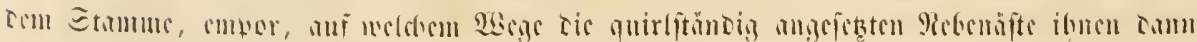
iptroulid) ioligen.

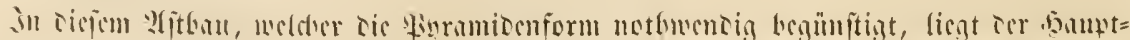

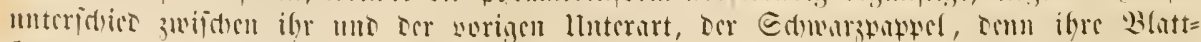

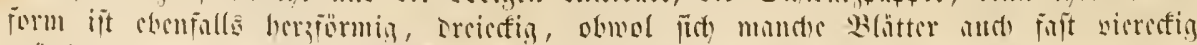

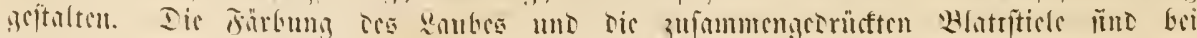





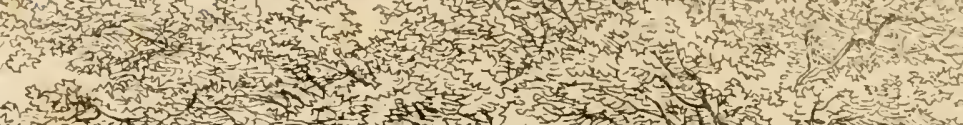

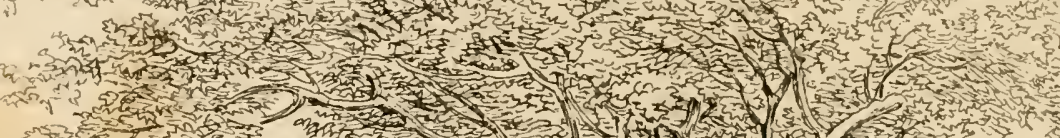

क

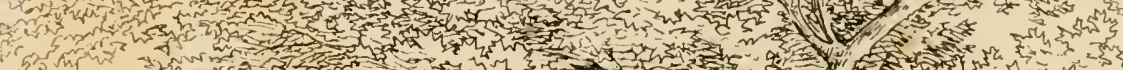

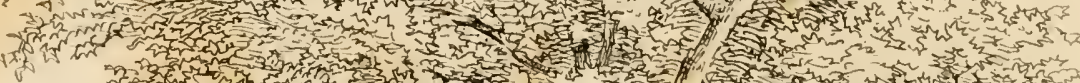
A

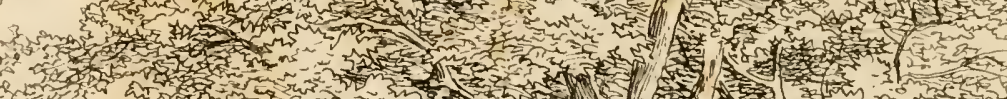

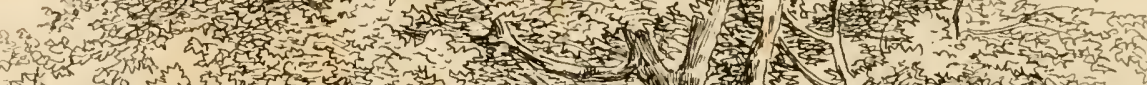
Fh

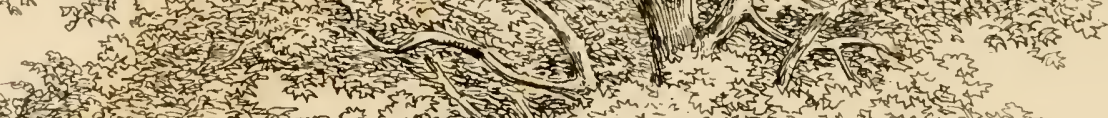
m.

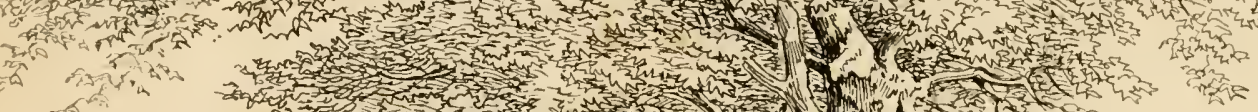

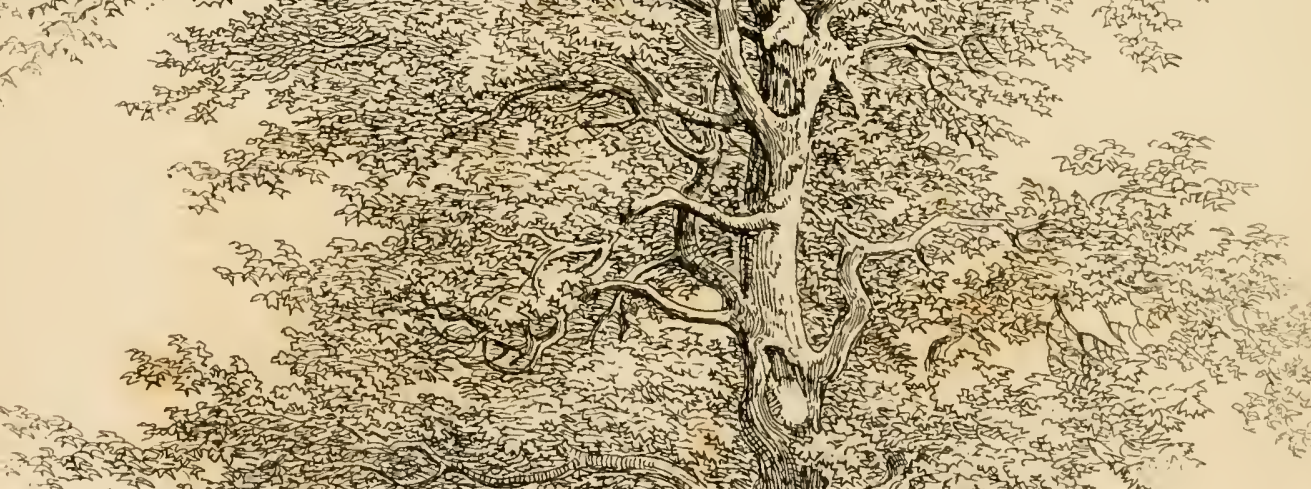

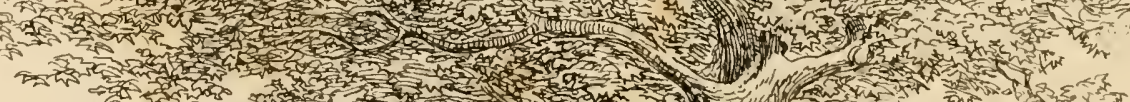

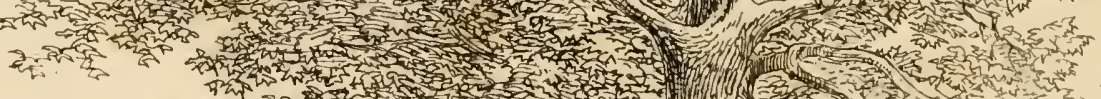

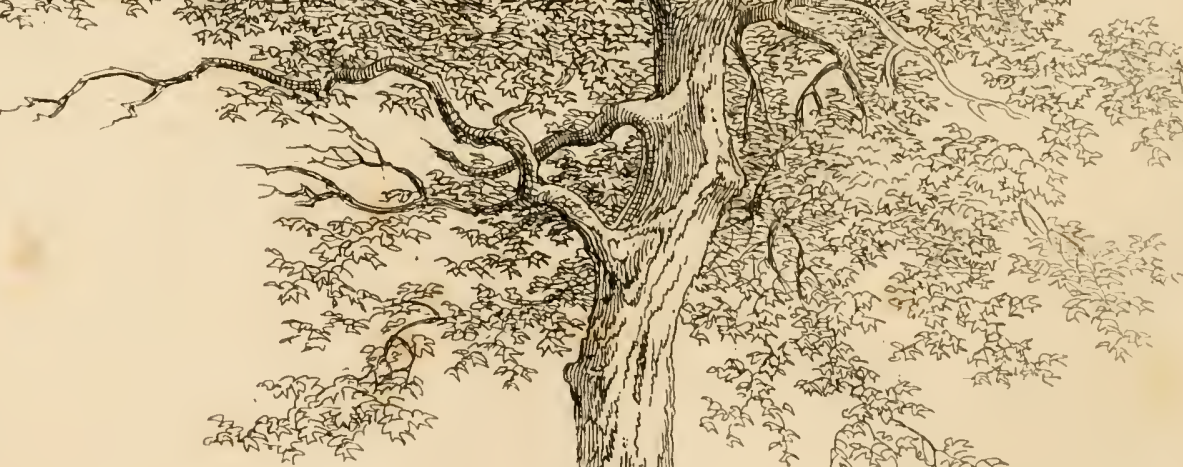




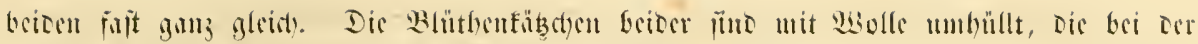

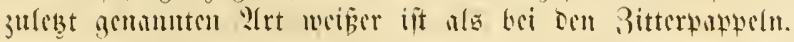

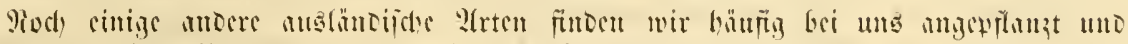

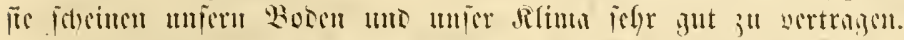

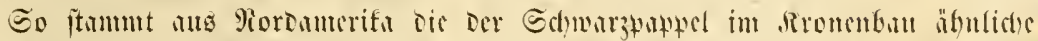

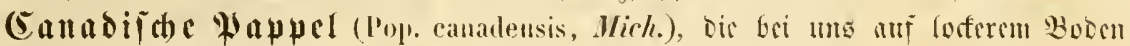

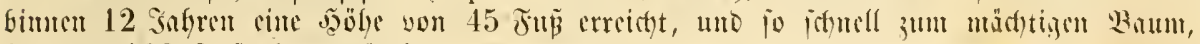

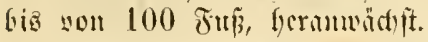

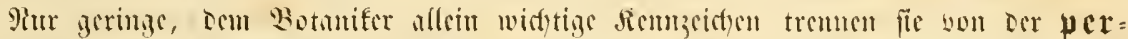

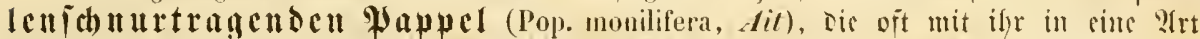

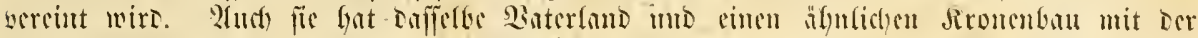
norigert.

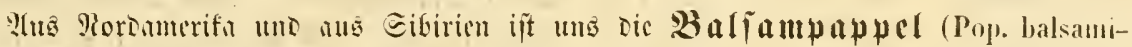

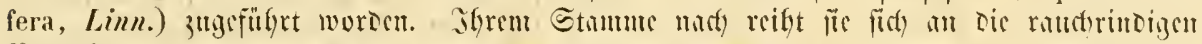
Fitpeln an.

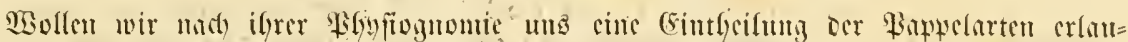

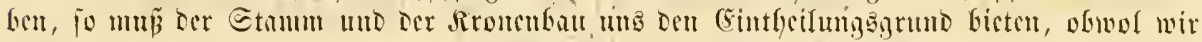

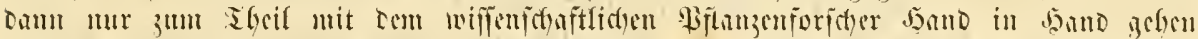

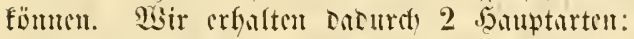

\begin{tabular}{|c|c|c|}
\hline 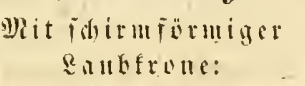 & $\begin{array}{l}\text { a. Wit foirmfurmiger } \\
\text { : Rrume: }\end{array}$ & b. Finamiosuform: \\
\hline 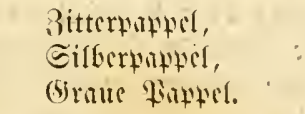 & 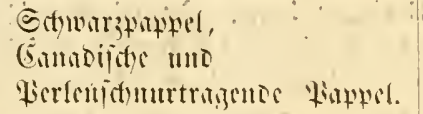 & Strticuitade Pirpte \\
\hline
\end{tabular}

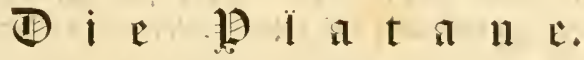

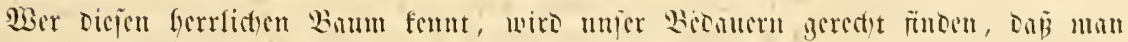

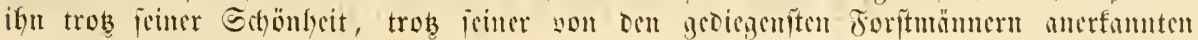

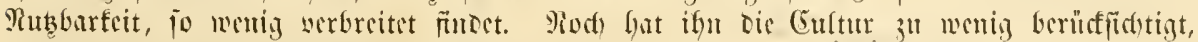

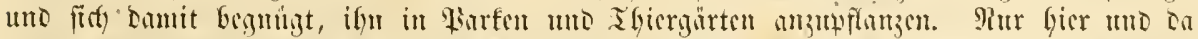

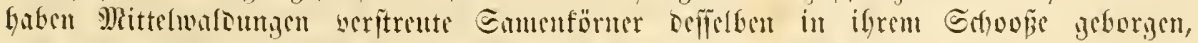

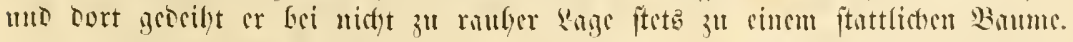

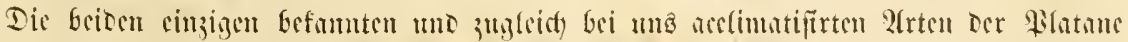

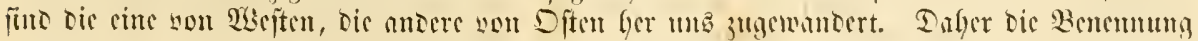

oer morbculändifdyen mit

Der abenofandifach Iilatane.

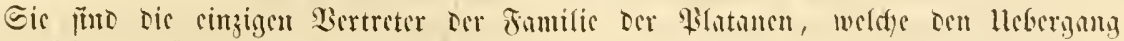

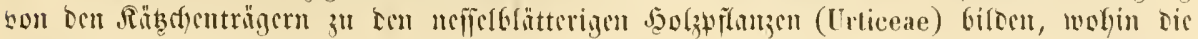

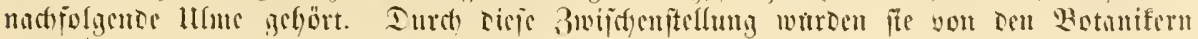

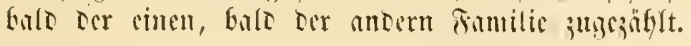




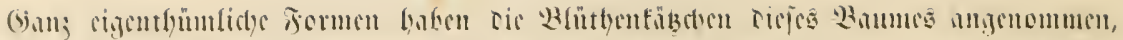

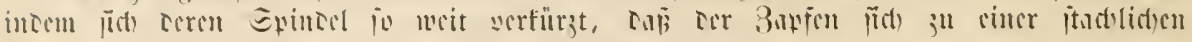

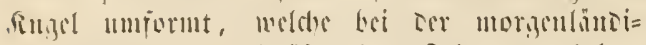

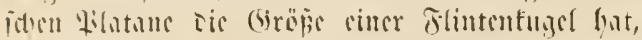

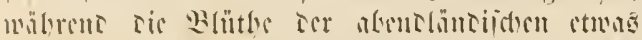

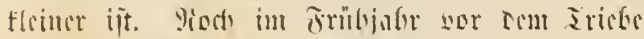

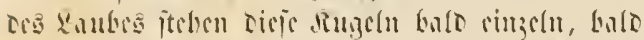

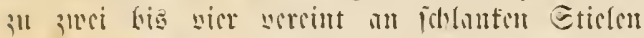

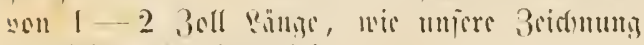

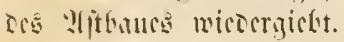

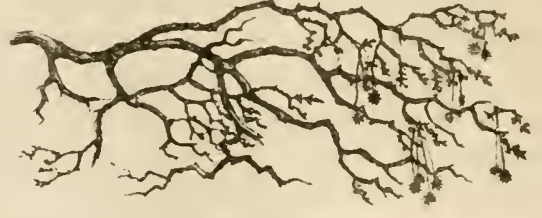

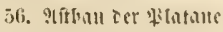

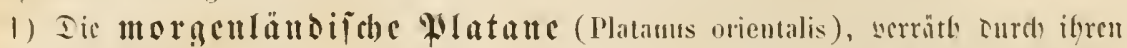

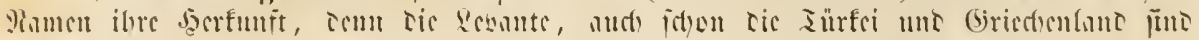

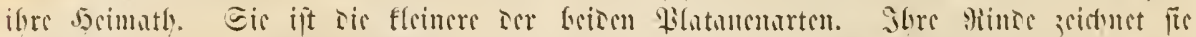

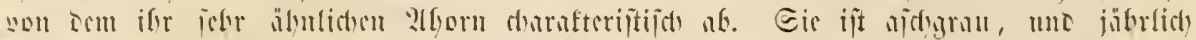

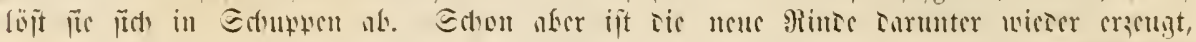

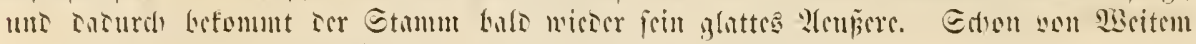

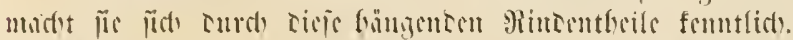

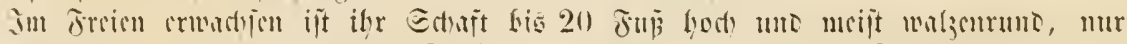

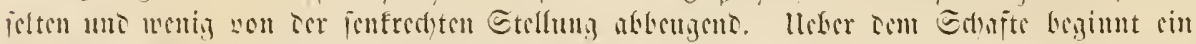

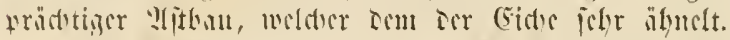

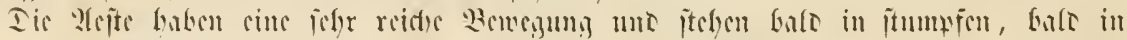

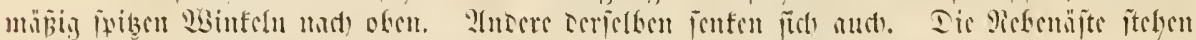

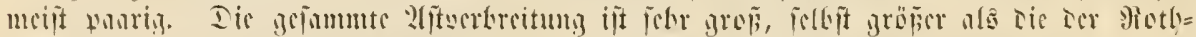

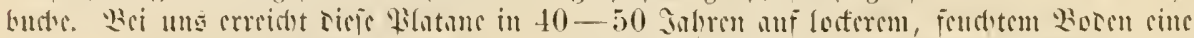

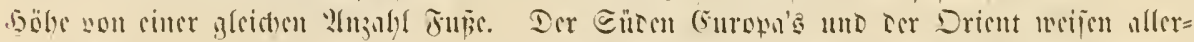

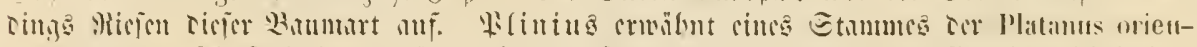

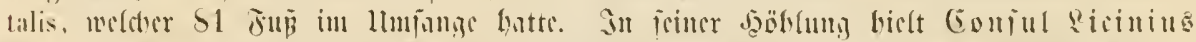

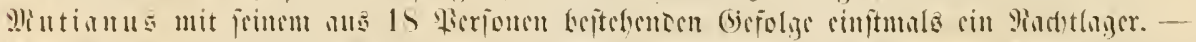

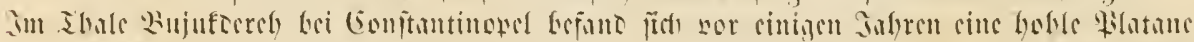

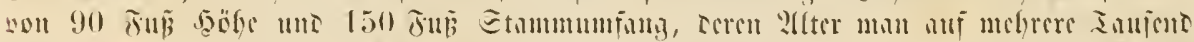

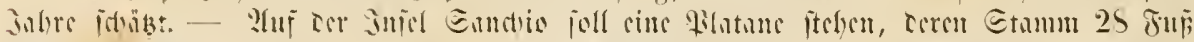

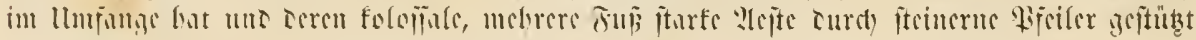
merien müntes.

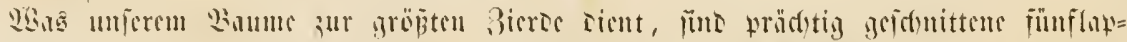

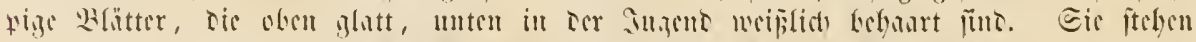

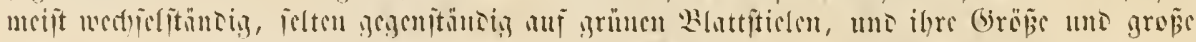

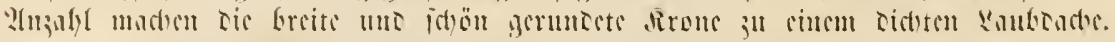

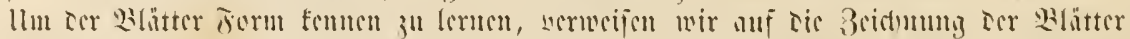

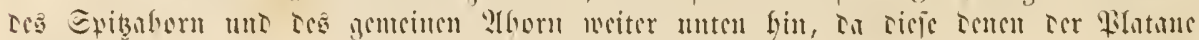
ylcitich.

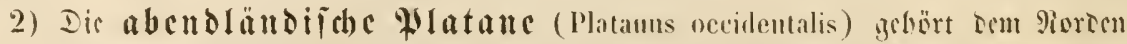

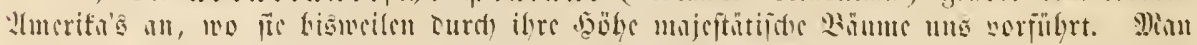

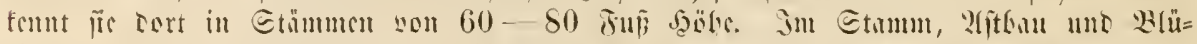

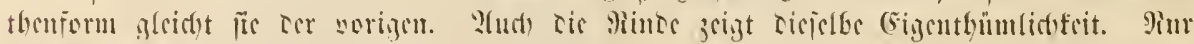

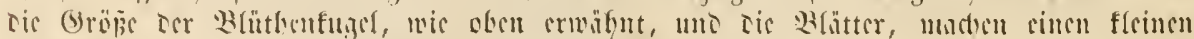

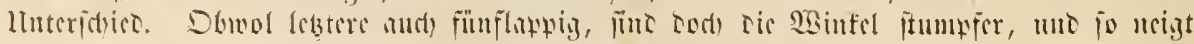

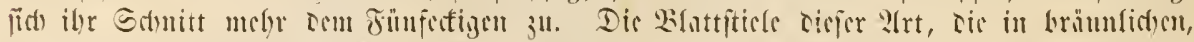

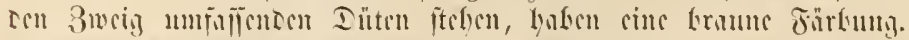

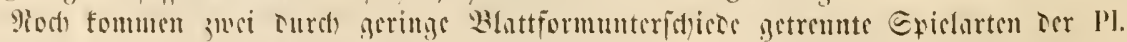
oreidentalis in unjeren (Bïrten bor. 



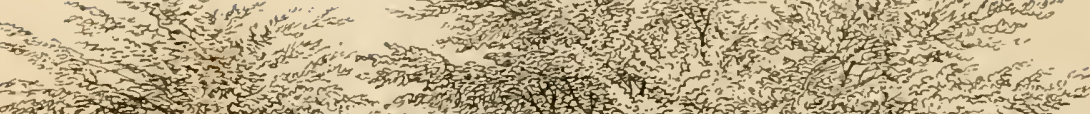

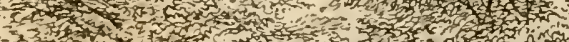

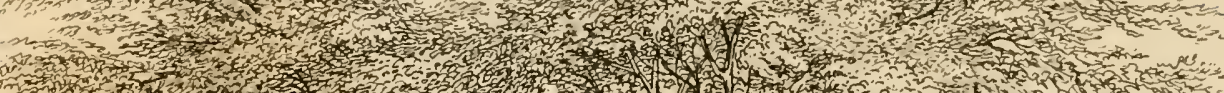

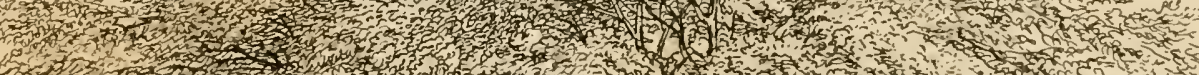

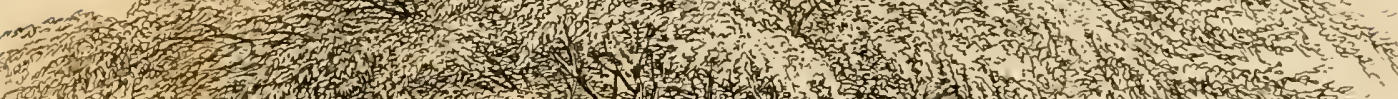

(1)

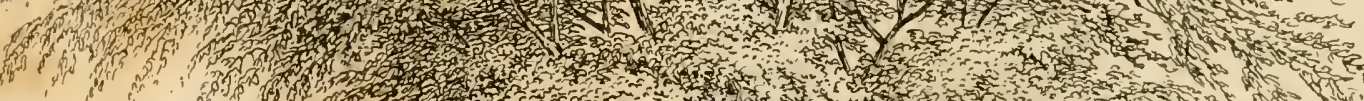

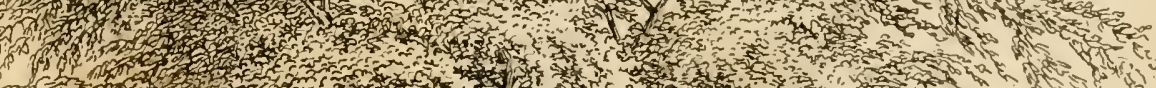

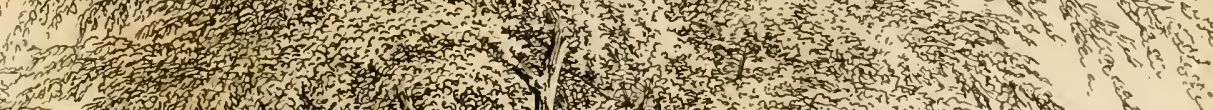

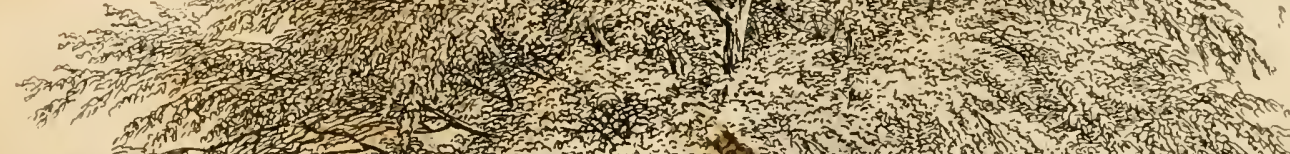

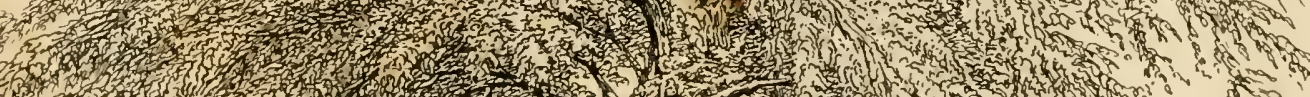
mon,

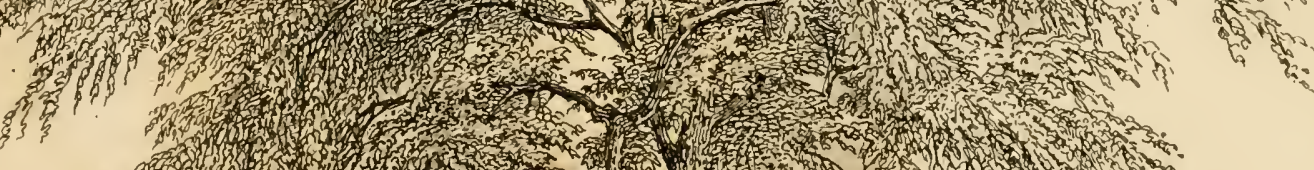

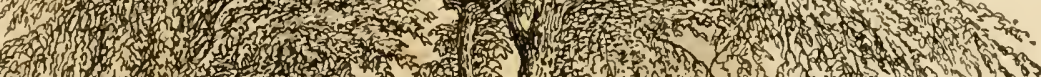

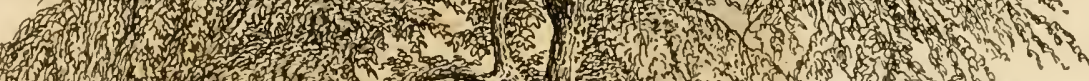

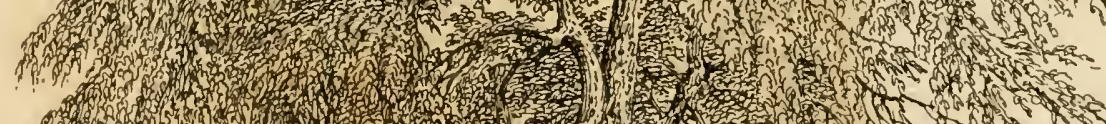

m.9.7.6.

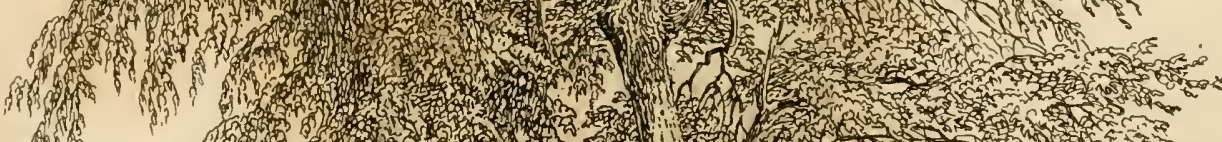

(1)

if

of $0_{0}$.

月.

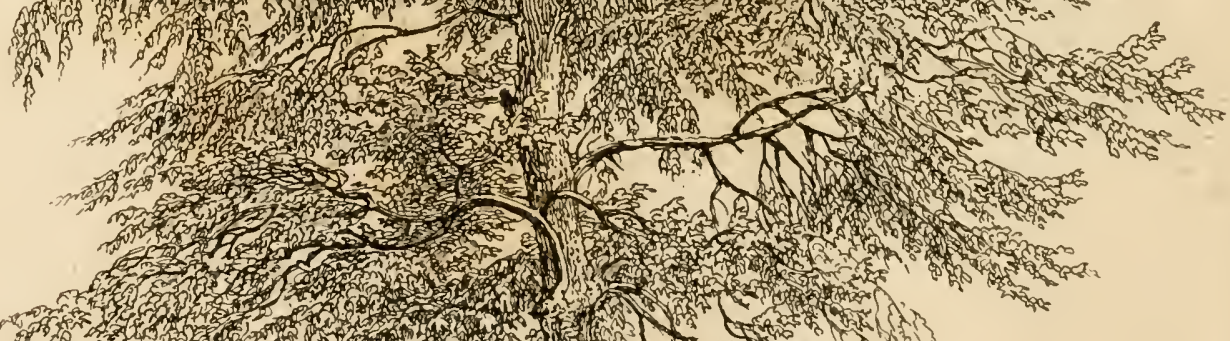

mor.

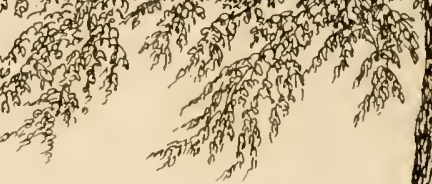

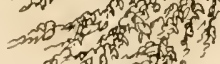

astis

(t)

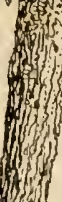

1 .

5. 6 .

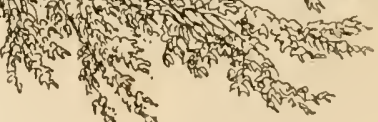




\section{(1) $i \mathfrak{i}$ ll $\mathrm{i}$ m $\mathfrak{i}$.}

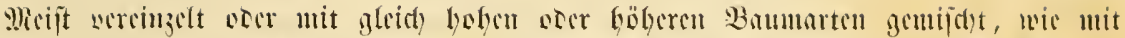

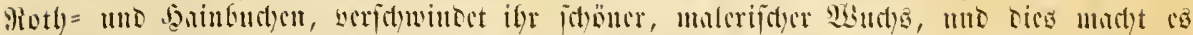

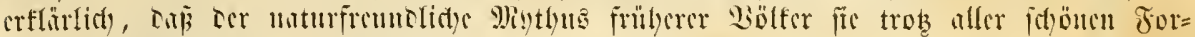

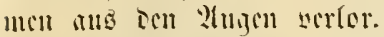

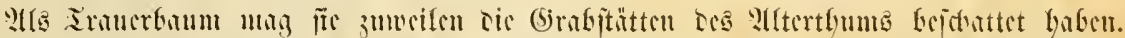

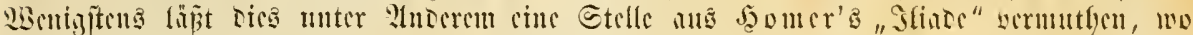

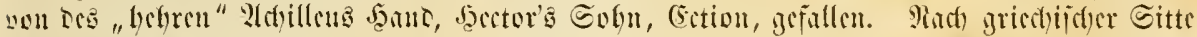

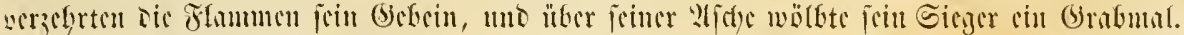

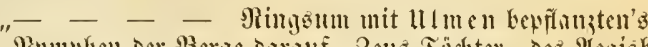

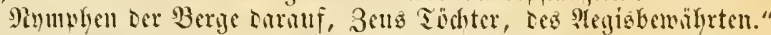

(5) 1 m. SI. VI, 419.)

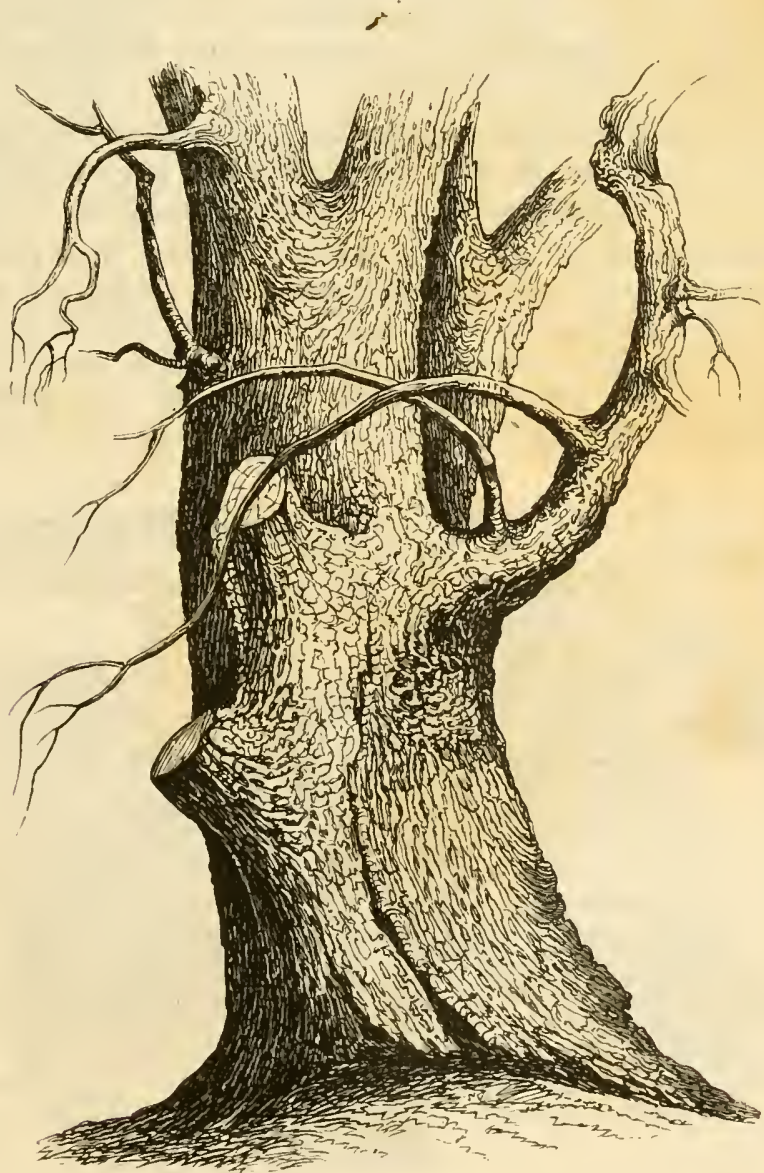

5. Uแmenitumม 


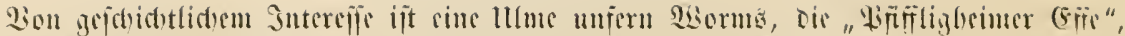

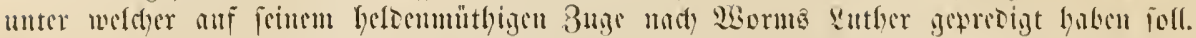

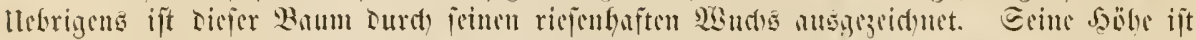

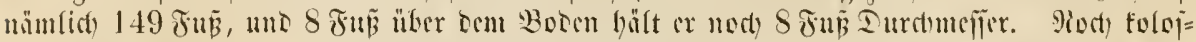

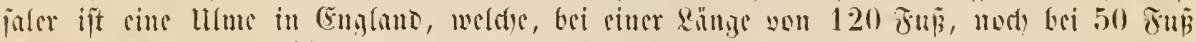

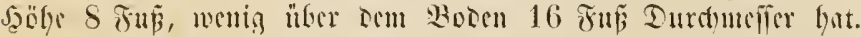

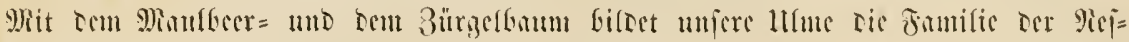
jelartigen (Crticeate) ooer oor lllmacen.

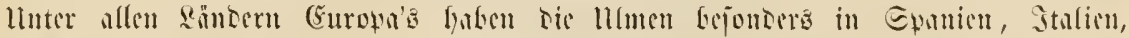

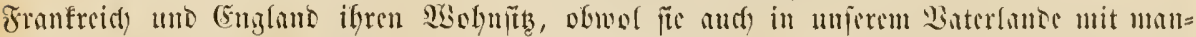

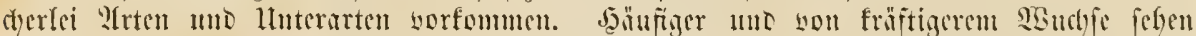

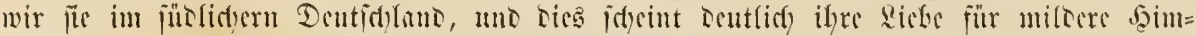

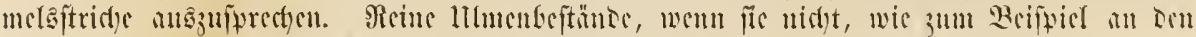

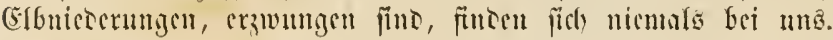

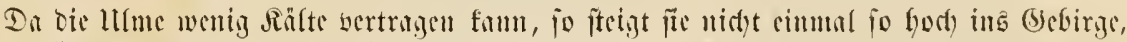

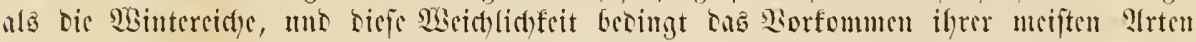

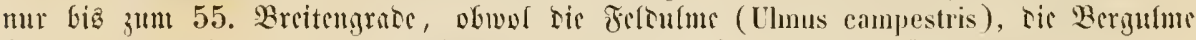

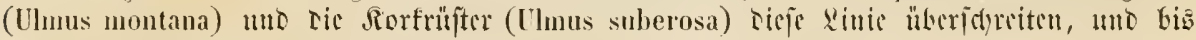
zum 60. (S)ris und nod) Darüber worfommen.

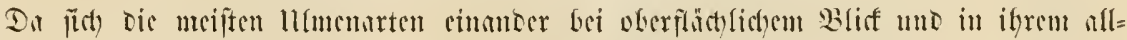

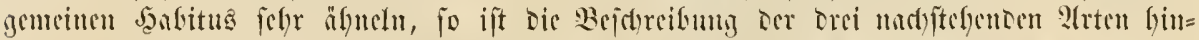

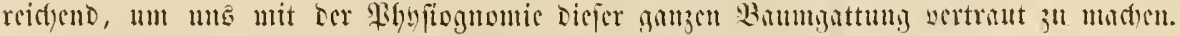

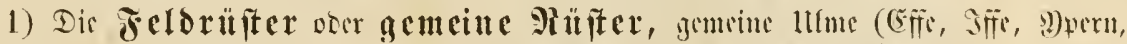

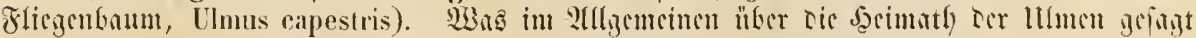

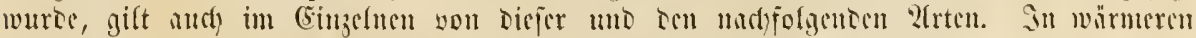

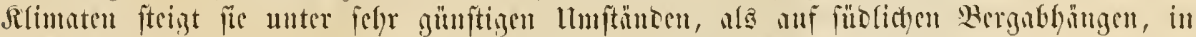

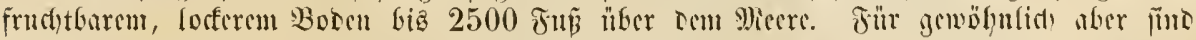
(56)

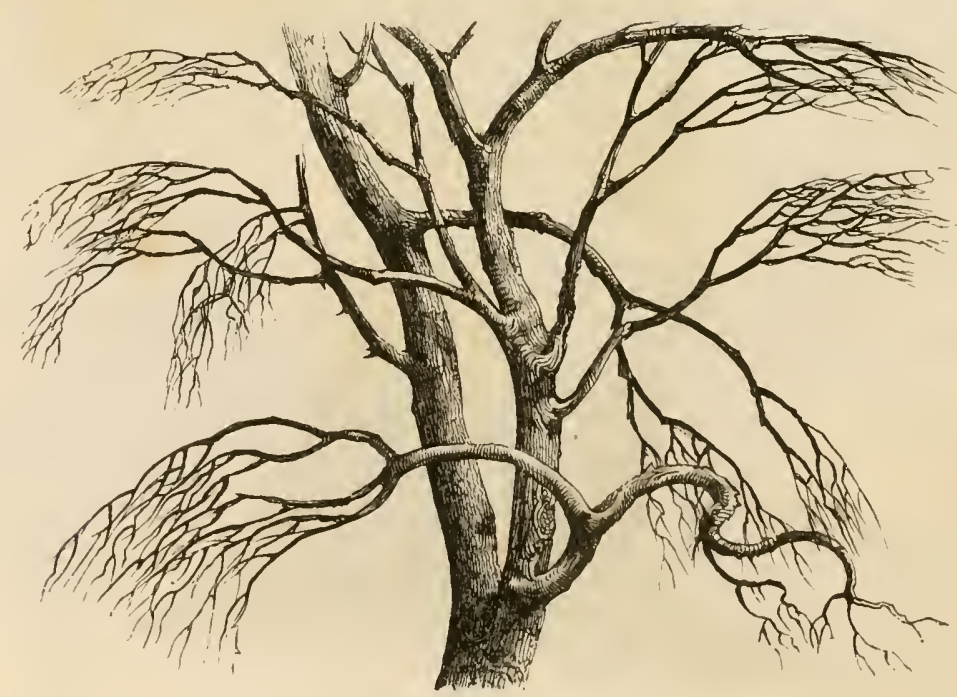

5x. 2litbu ber Müitor. 


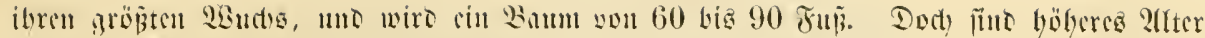

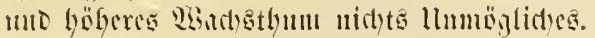

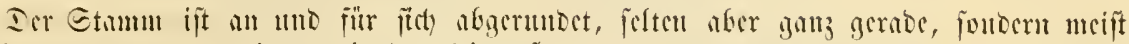

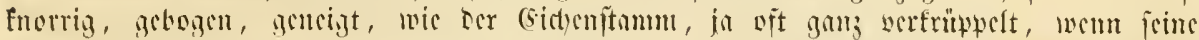

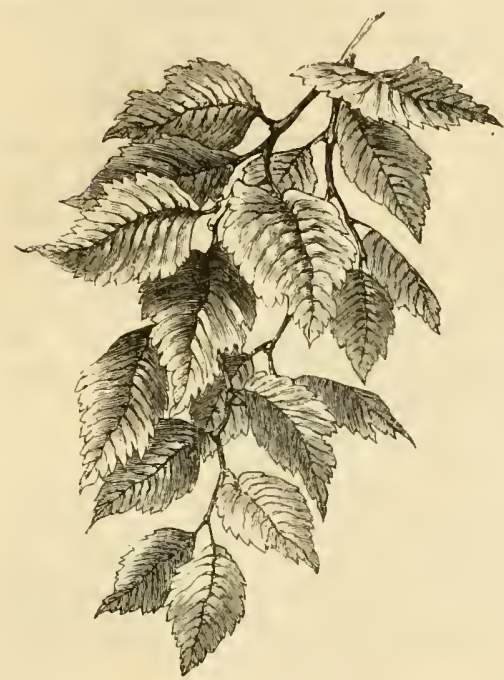

59. Blattitumo rer Riititer.

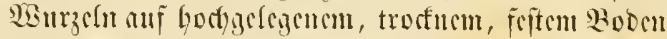

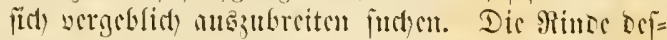
felben ift Dunfelgrnubrnum, uno je alter Der Gtamm,

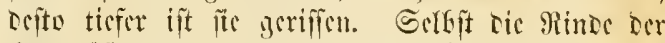

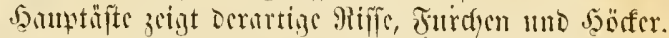

Saluer ift ca, con Pormaldarafter ifores Rro=

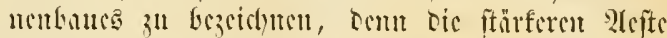
ftreben mebr nad) oben, als wollten fite fiet) zu ciner

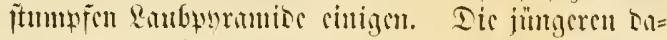

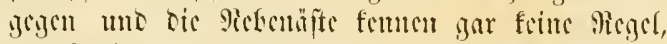

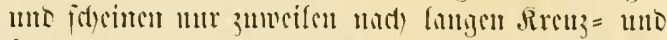
Anerjügen an Der Brujt oor mütterfiden ErTe ru= fon zu wollen, oer fie fïd) mit Ien jümgeren siric=

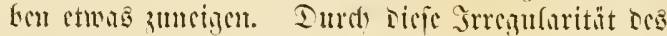

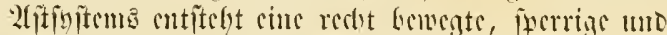

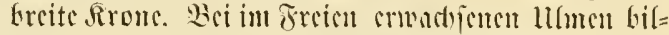

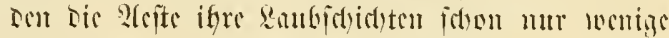

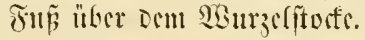

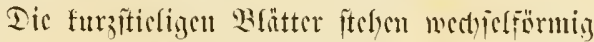

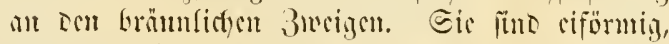

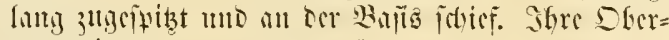

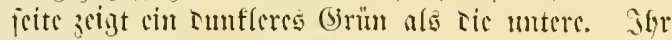

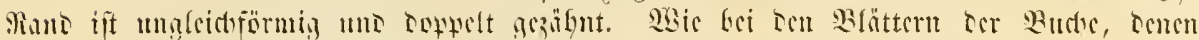

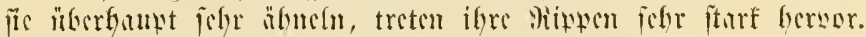

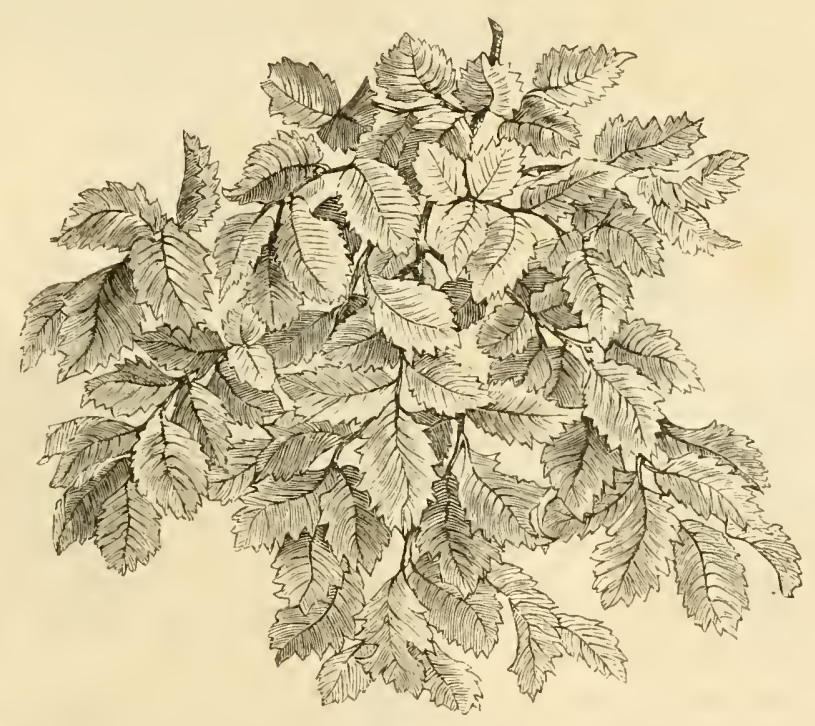

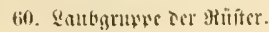




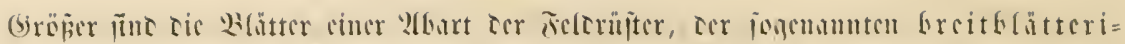

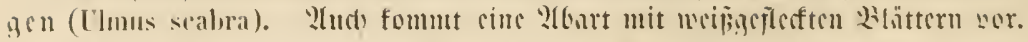

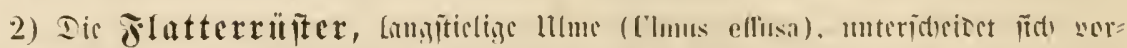

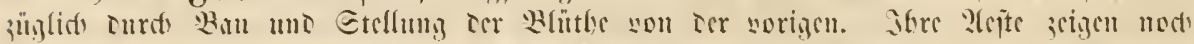

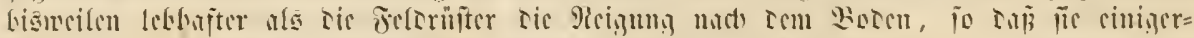

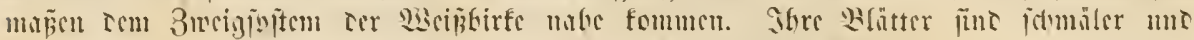

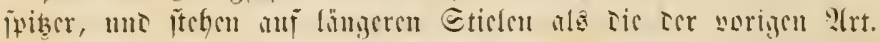

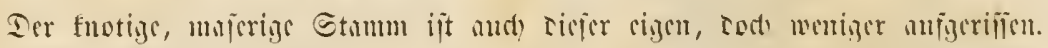

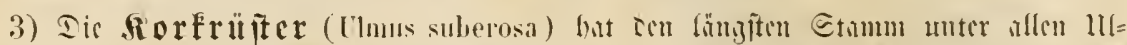

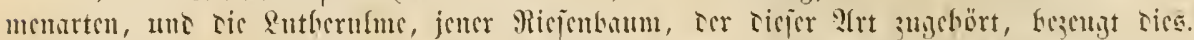

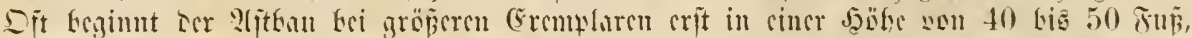

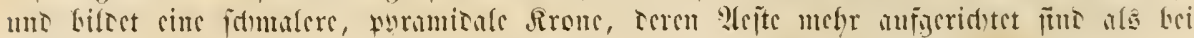
[en norigen 2 rentu.

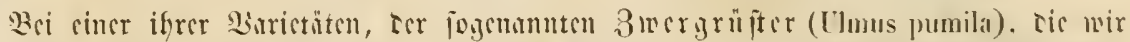

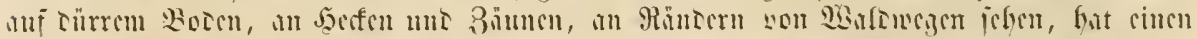

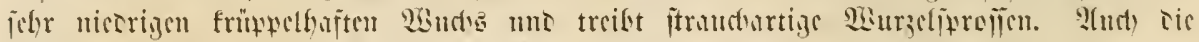

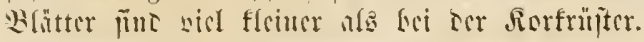

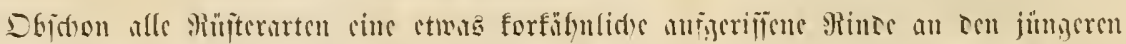

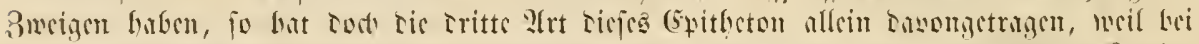

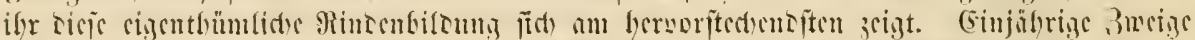

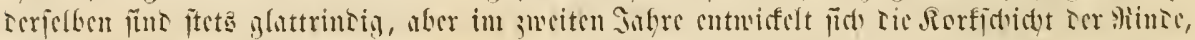

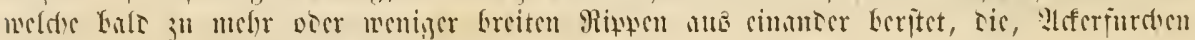

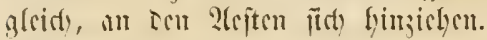

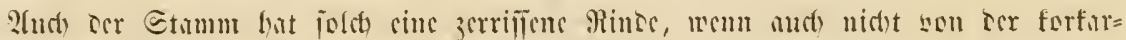

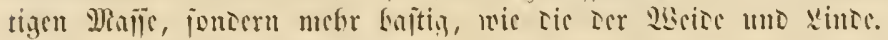

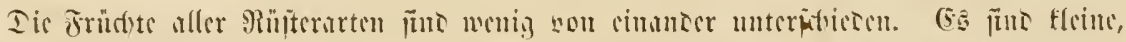

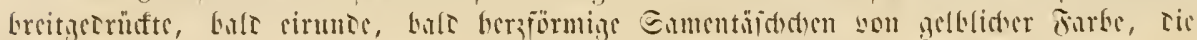

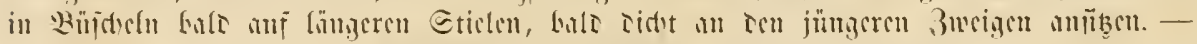

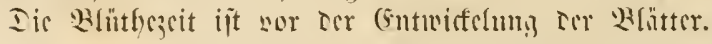

\section{D) $\mathfrak{i} \mathfrak{i} \mathbb{E} \quad \mathfrak{i} \mathfrak{i} \mathfrak{j} \mathfrak{i}$.}

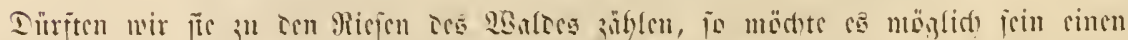

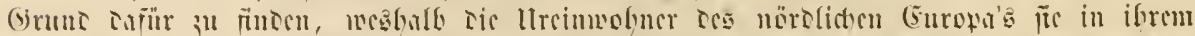

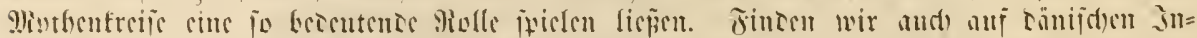

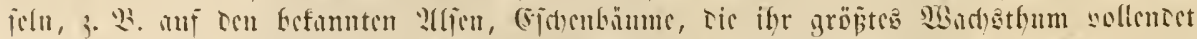

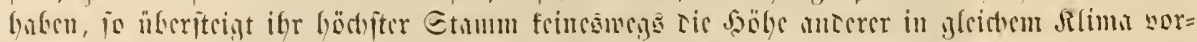

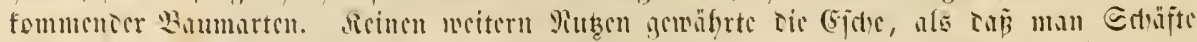

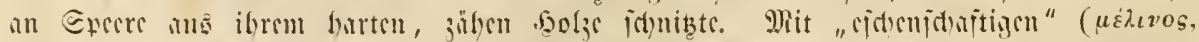

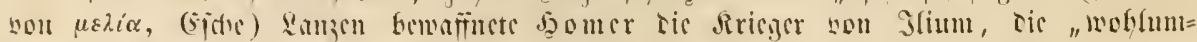

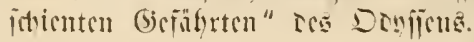

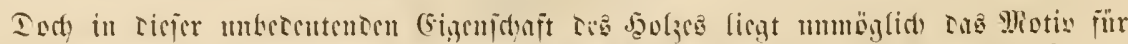

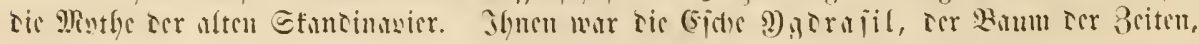




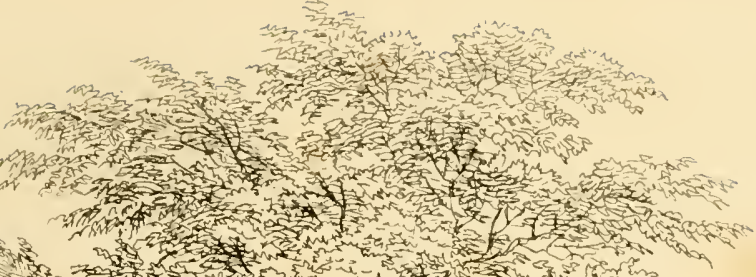

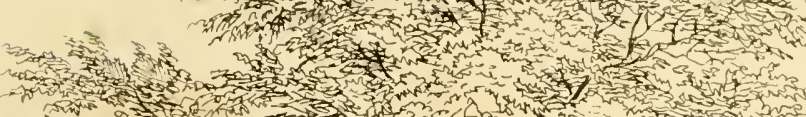

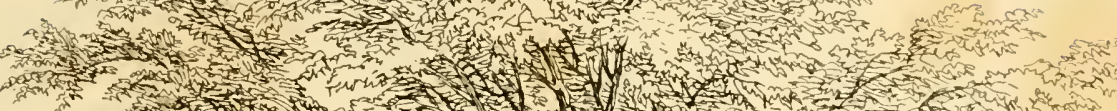

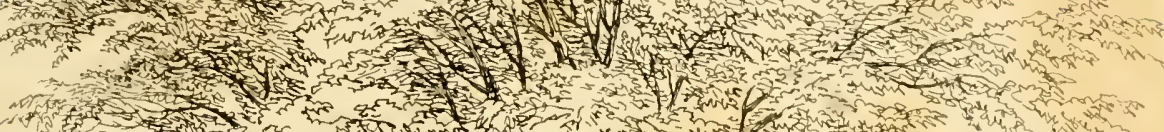

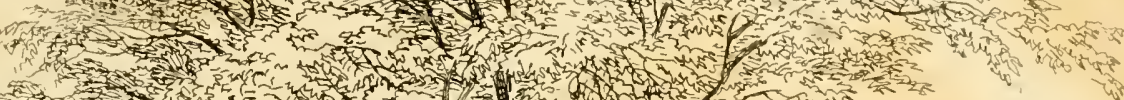
min

3.

1) 2 (2)

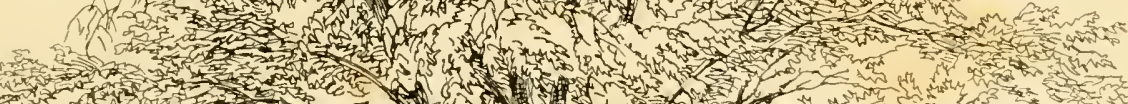

दf

mar.

mint

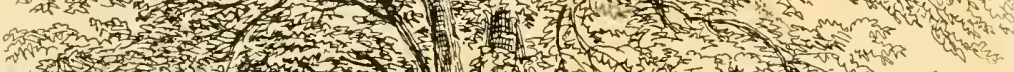

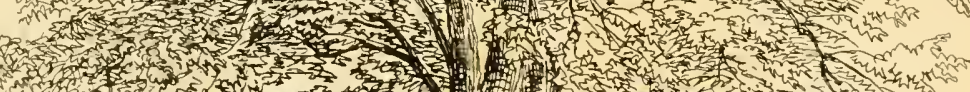

A

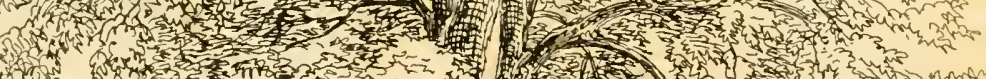

(ent

W.

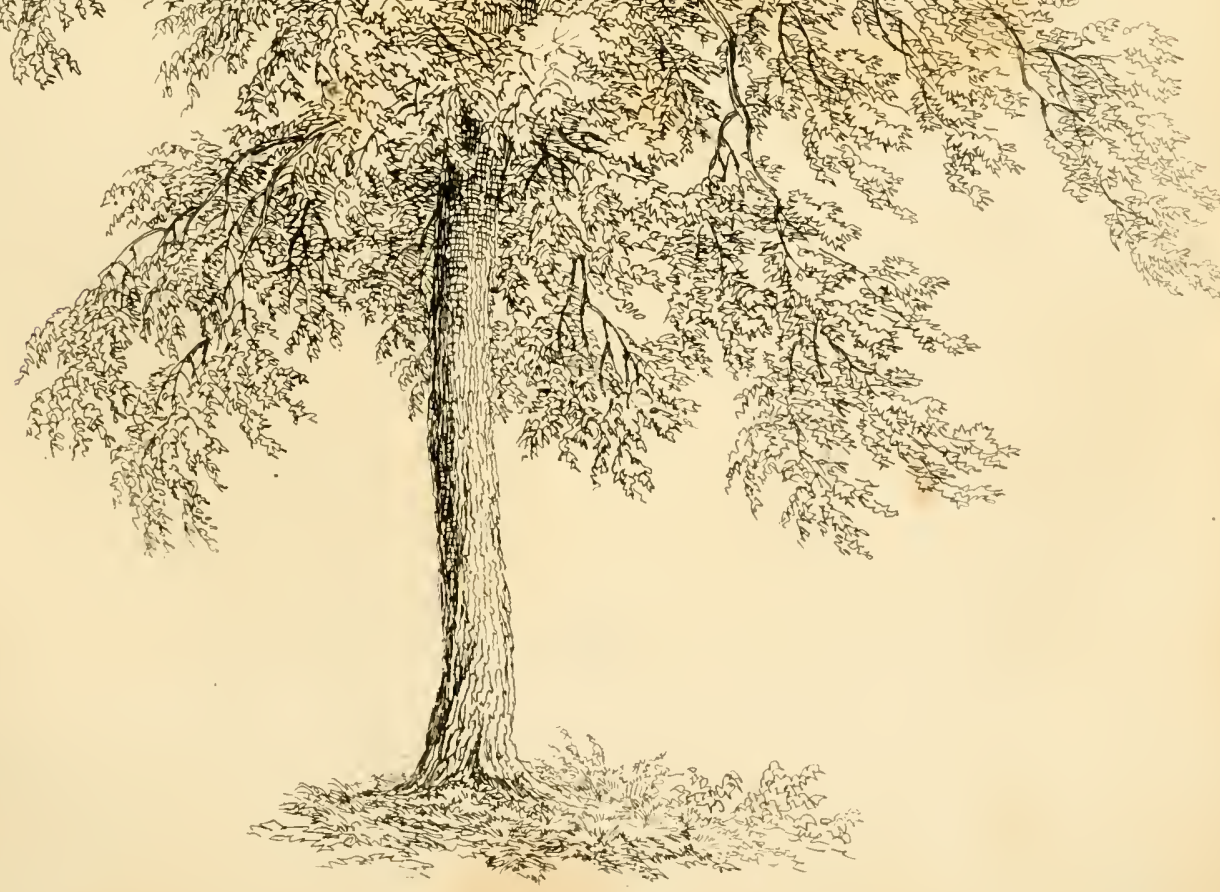




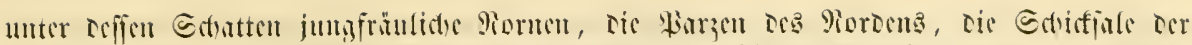

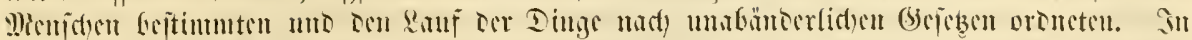

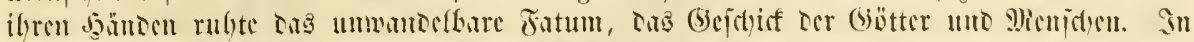

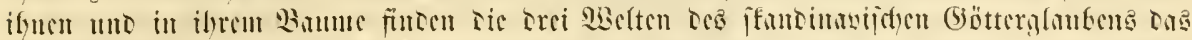

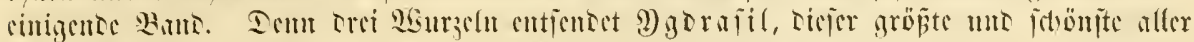

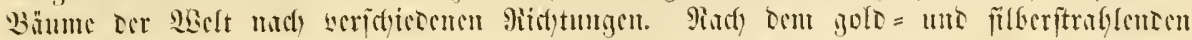

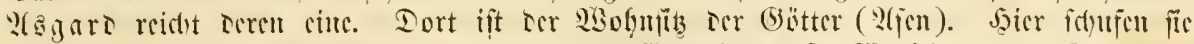

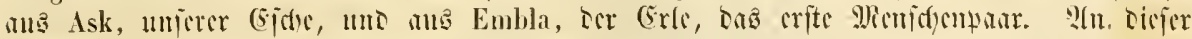

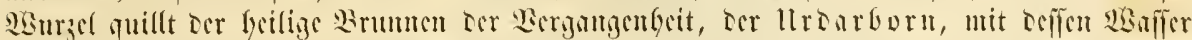

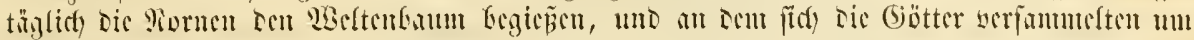
(baridyt ju balten.

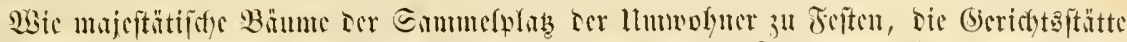

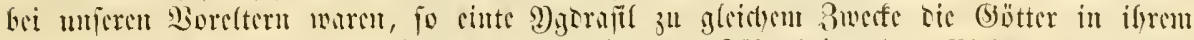

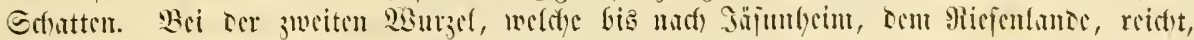

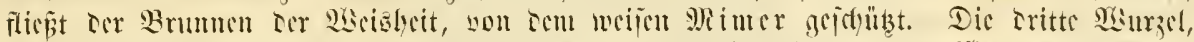

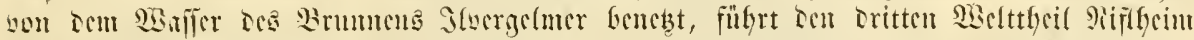

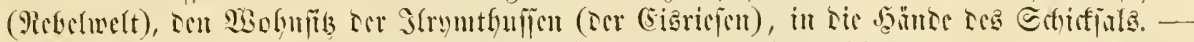

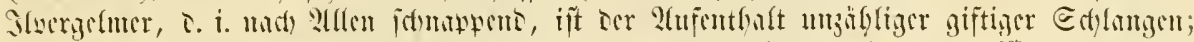

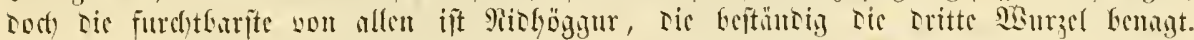

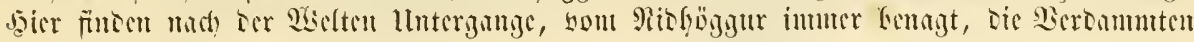
ilye Etrats.

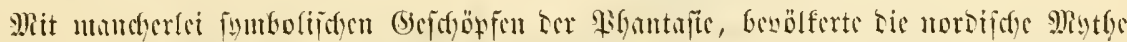

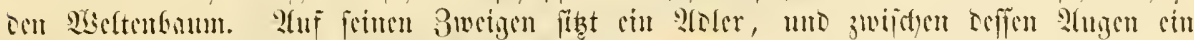

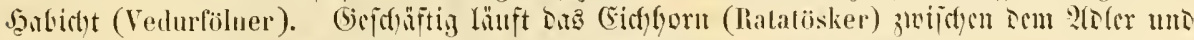

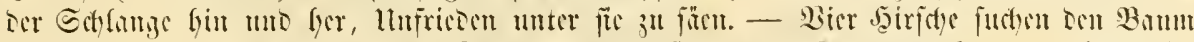

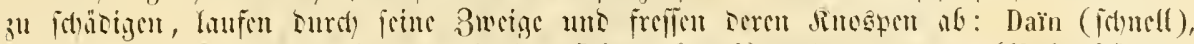

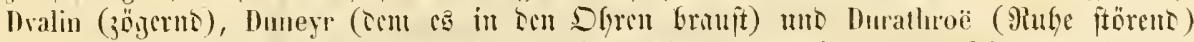

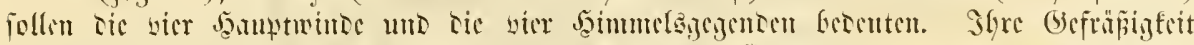

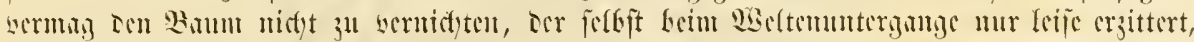

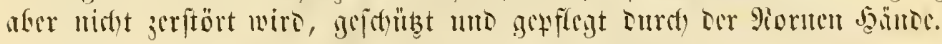

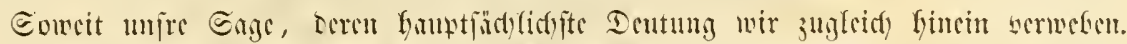

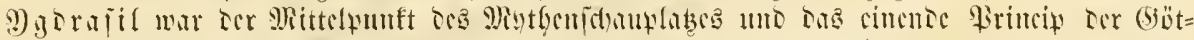

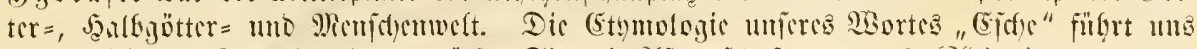

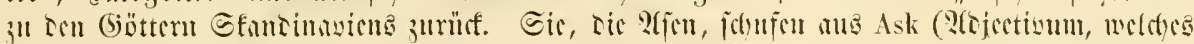

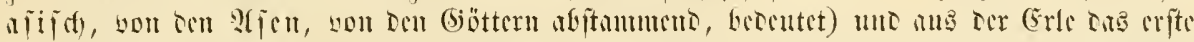

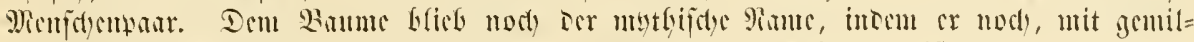

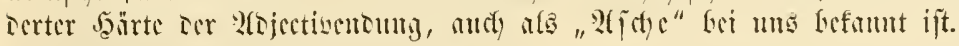

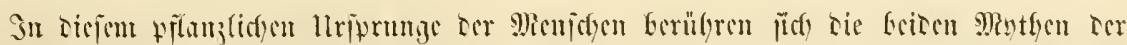

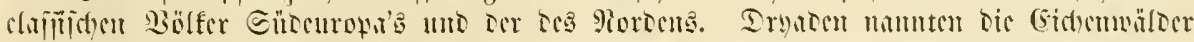

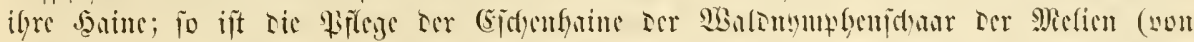

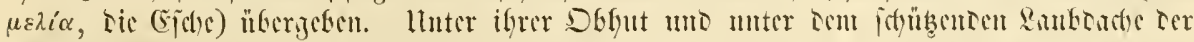

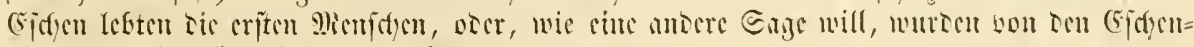
göttimen die erjêten Menjetyen geteren.

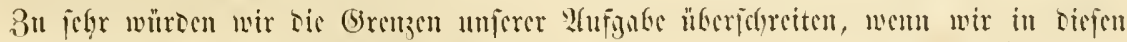

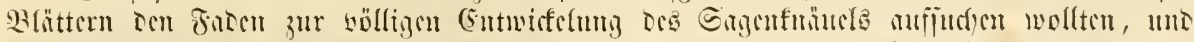

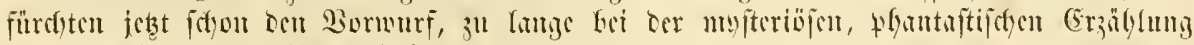
oer Rordinterer acrubeilt ju babent.

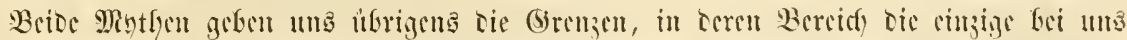

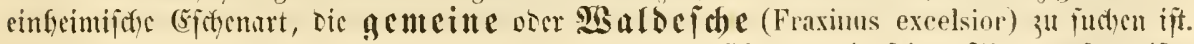

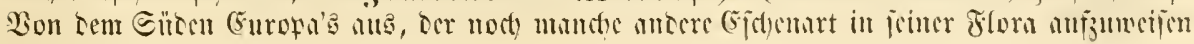




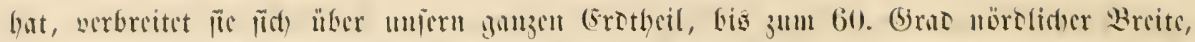

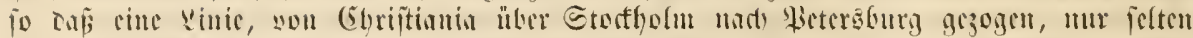

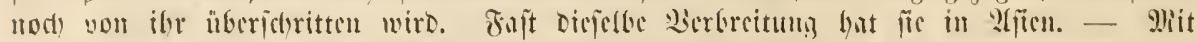

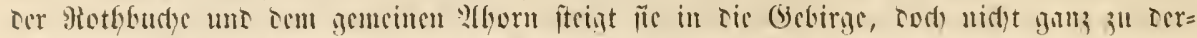

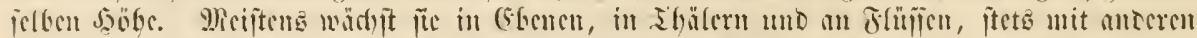

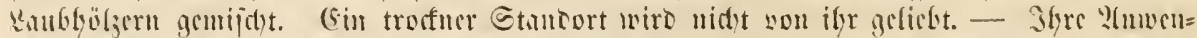

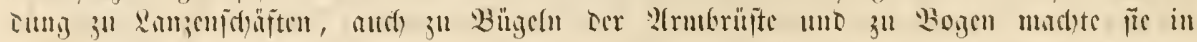

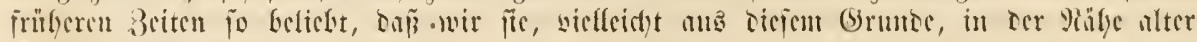
Burịgu of̈t f̈utos.

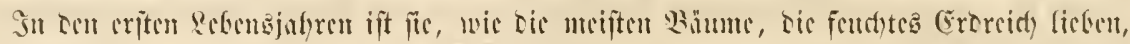

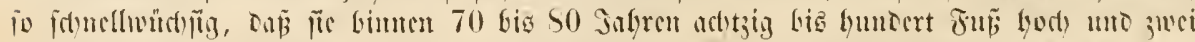

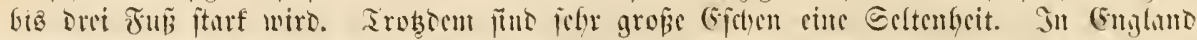

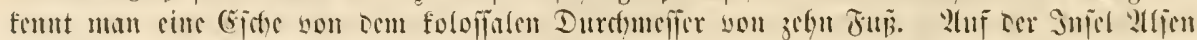

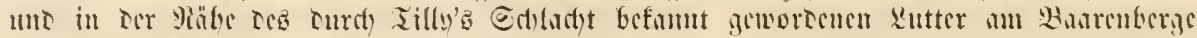
foumen iturfe હtümmic vor.

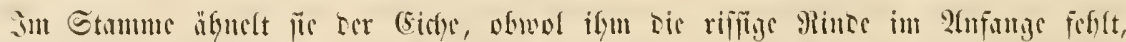

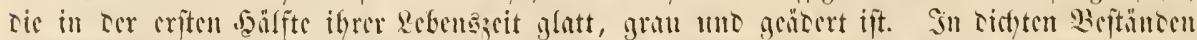

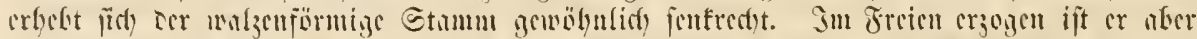

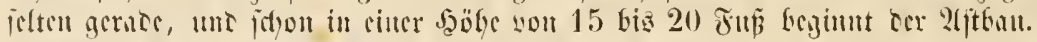

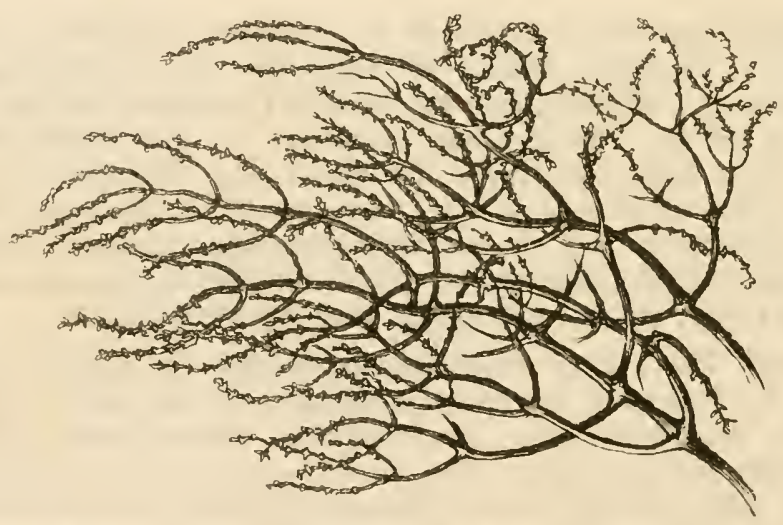

(i). Nithun ter Eidic.

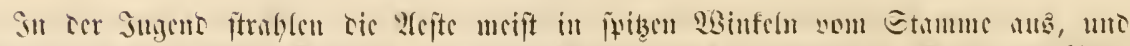

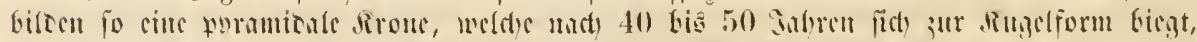

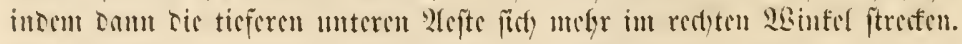

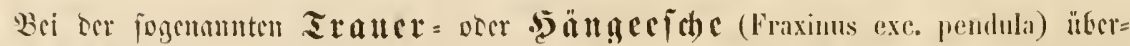

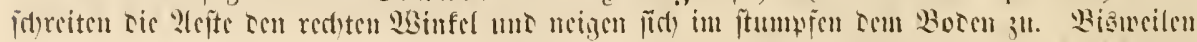

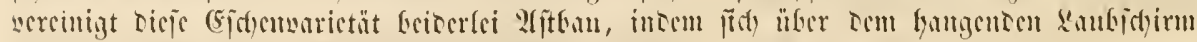

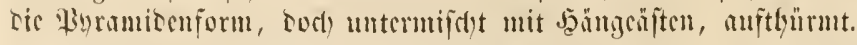

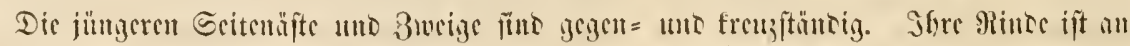

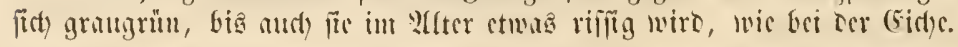

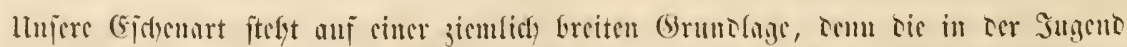

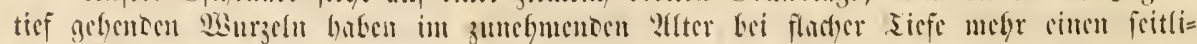
() 


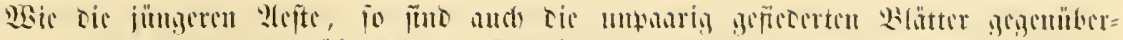

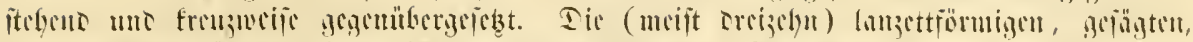

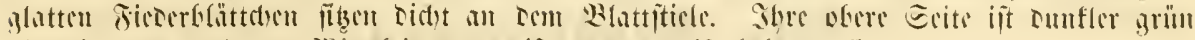

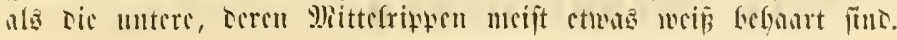

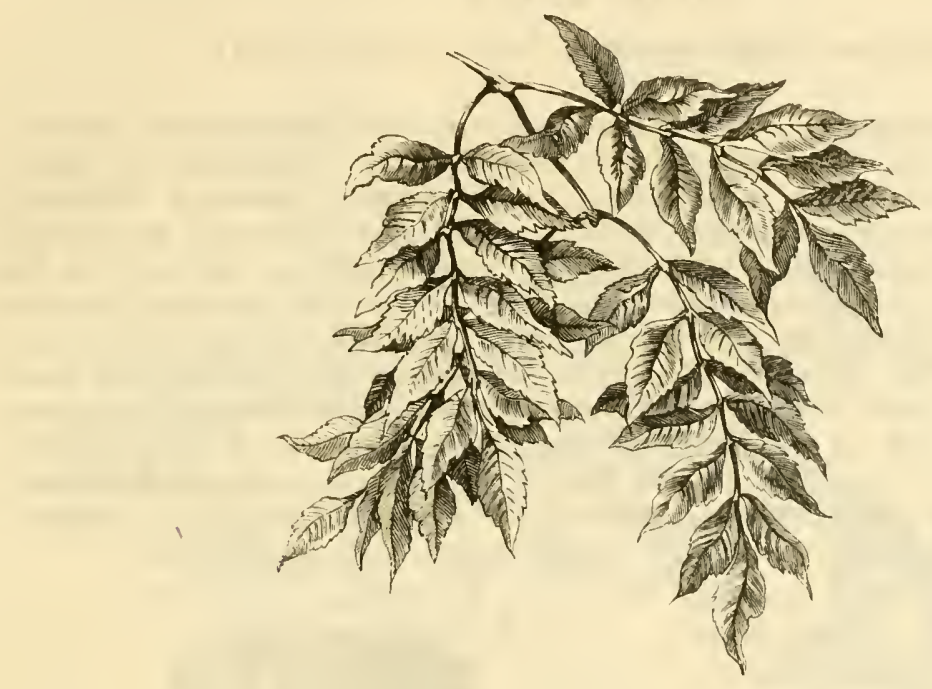

62. Blattiftuto Der (Eid)c.

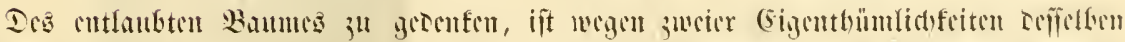

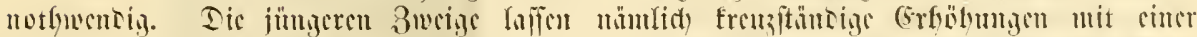

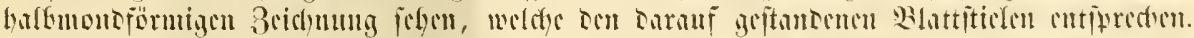

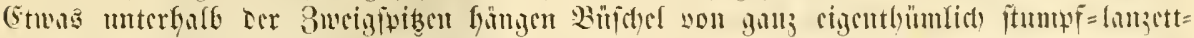
förming geitalteten Samenfot)oten.

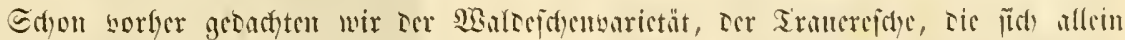

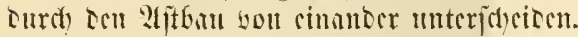

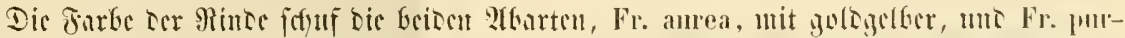

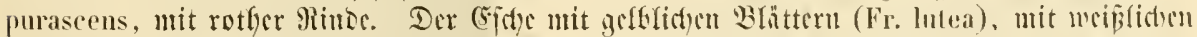

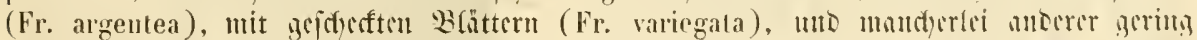

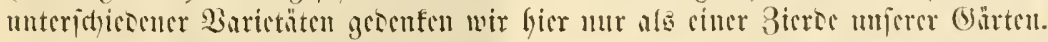

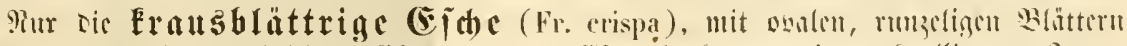

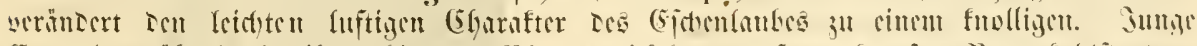

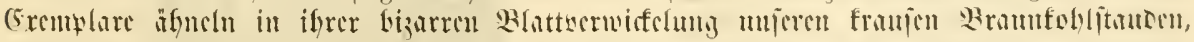

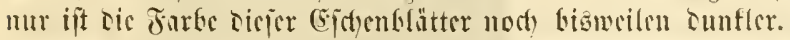

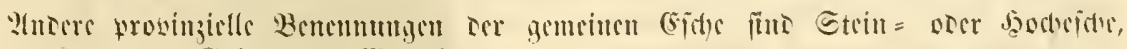

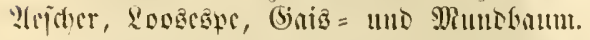




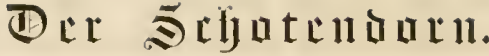

\author{
[Falide alfajic.]

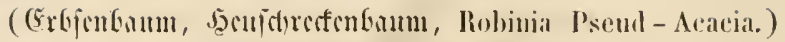

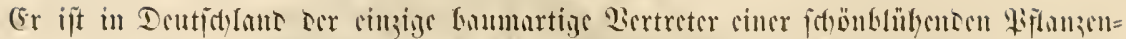

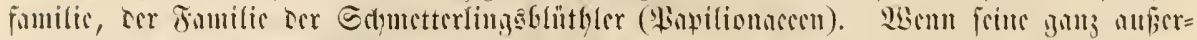

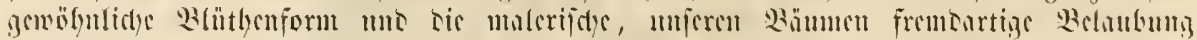

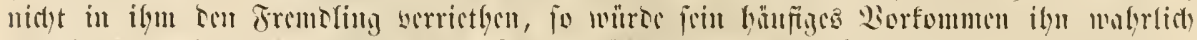

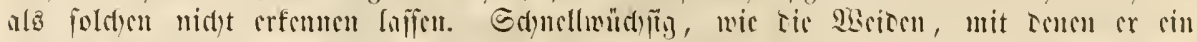

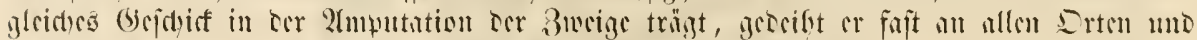

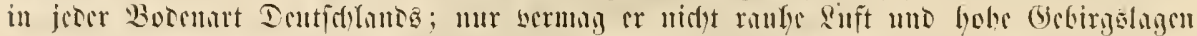

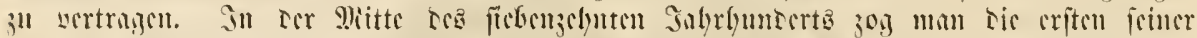

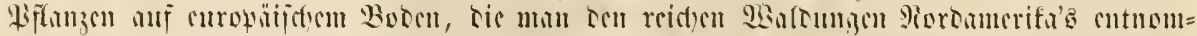

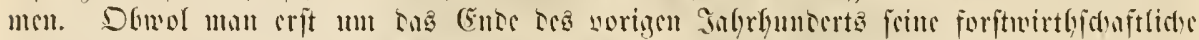

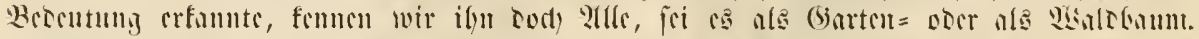

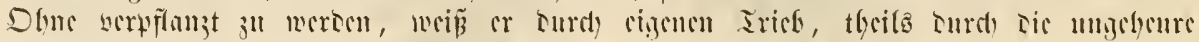

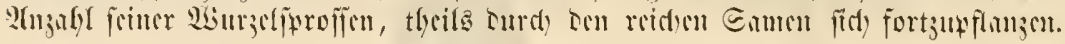

Selten menigt or fits in Soctumalo=

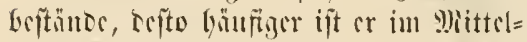

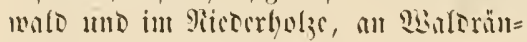

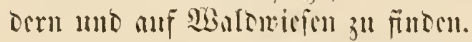

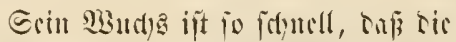

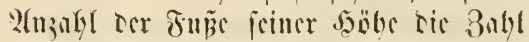

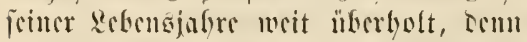
it 30 bis 40 Solven wirt or jelbit foci

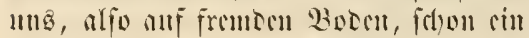

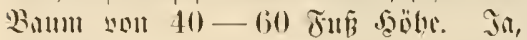

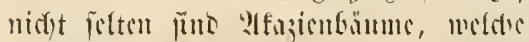

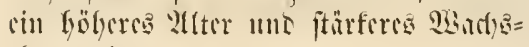
tbum zriģen.

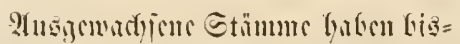

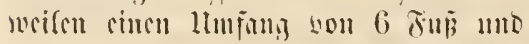

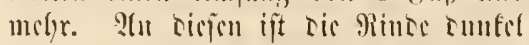

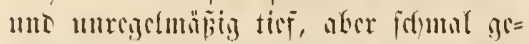

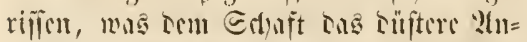

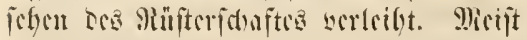
mad)jen fie follonf empor, nloce bïntis

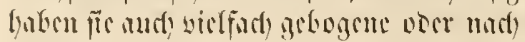

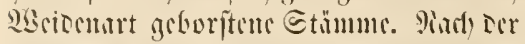

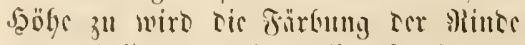
etmas lyeller mo tis grell gejofnittenen Rifife ctmas fladocr.

Bei cincm in Freicn ermathienen

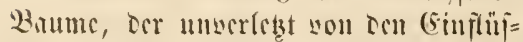

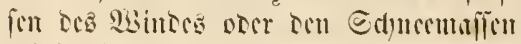

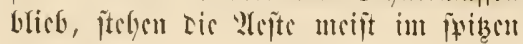

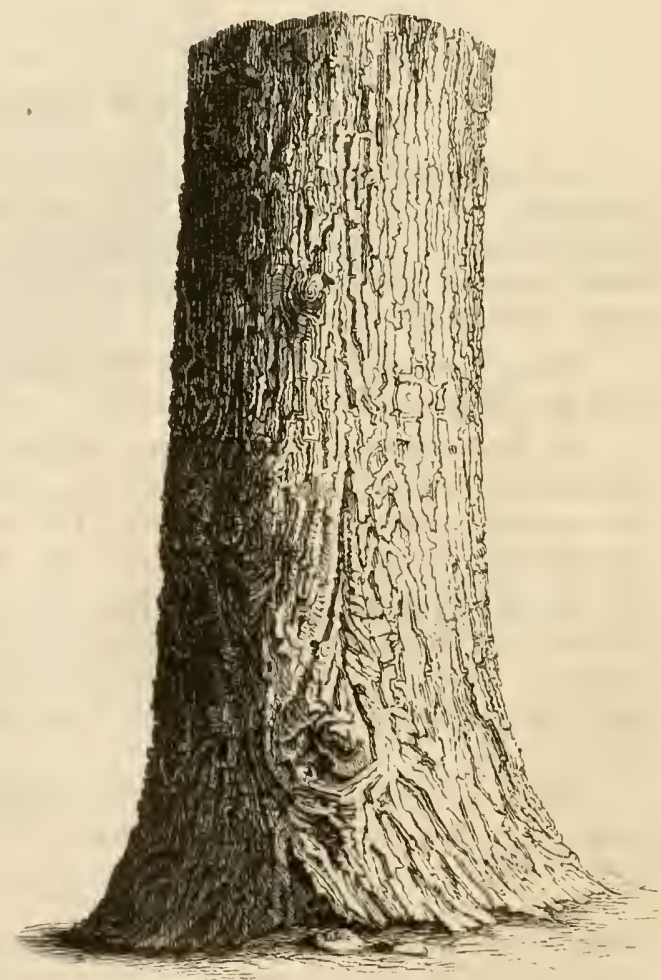

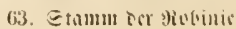




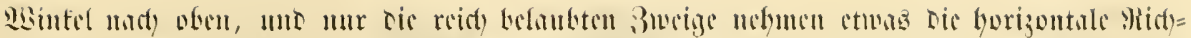

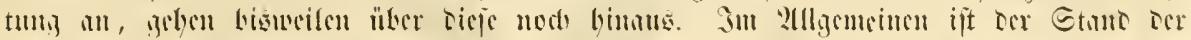

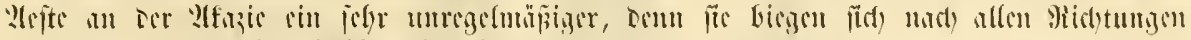

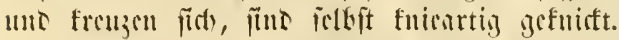

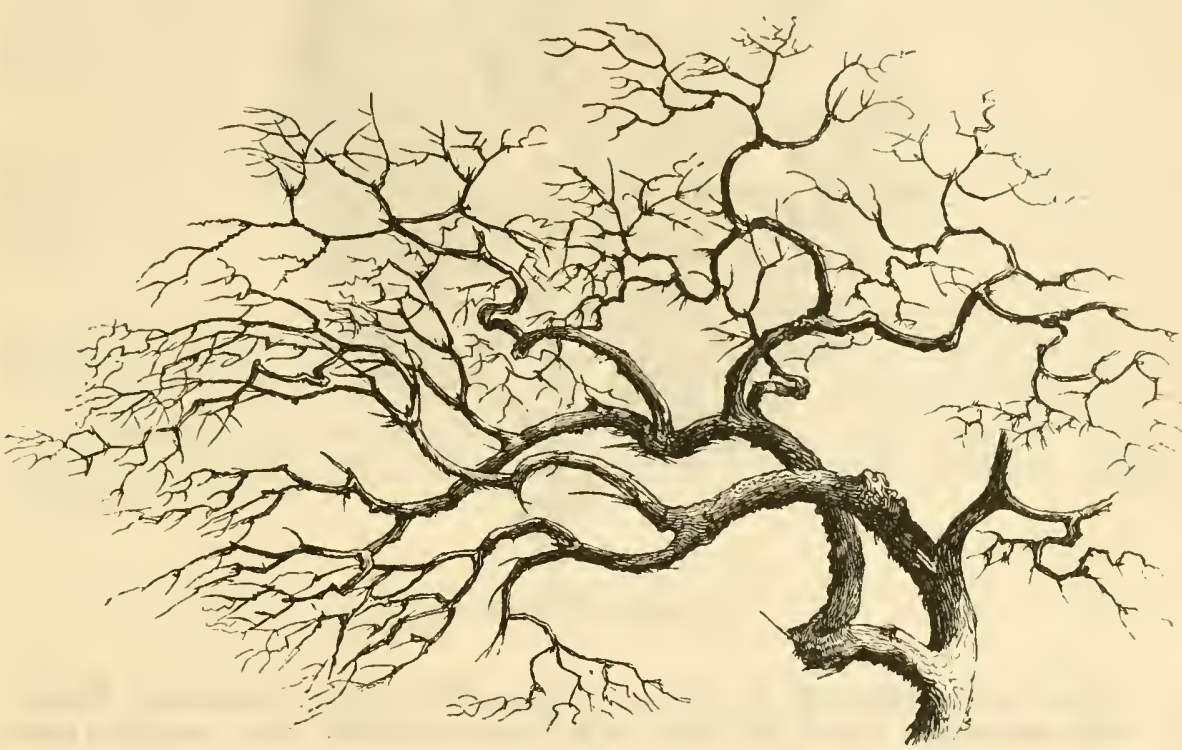

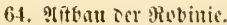

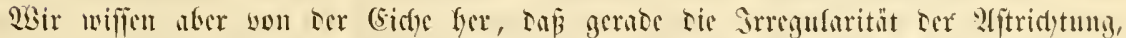

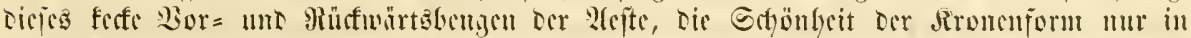

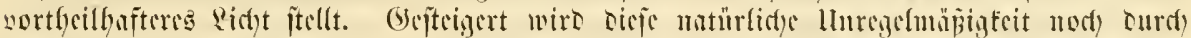

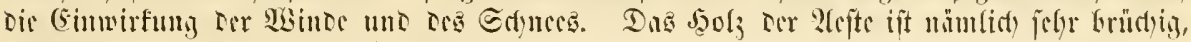

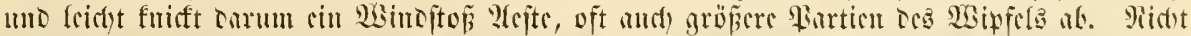

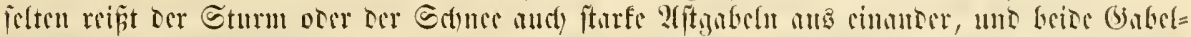

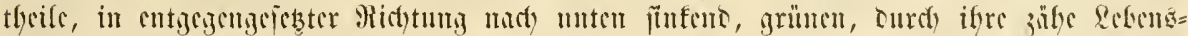

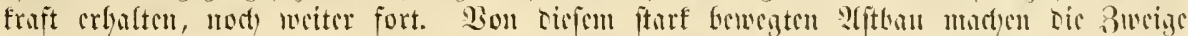

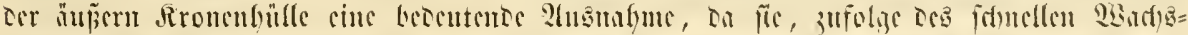

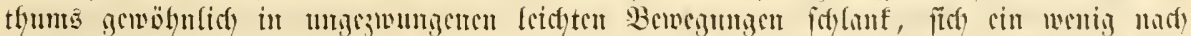
unteu jenfen.

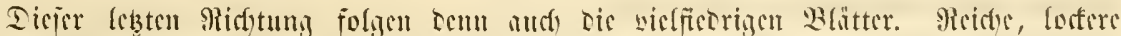

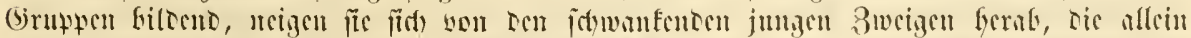

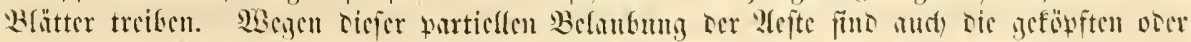

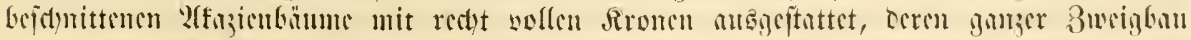

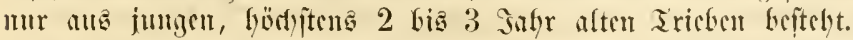

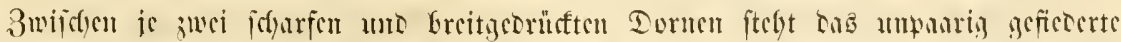

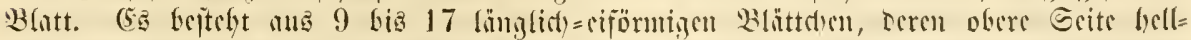

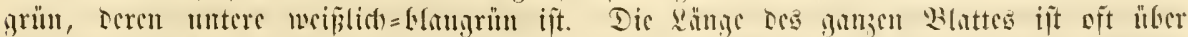

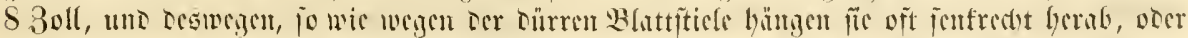

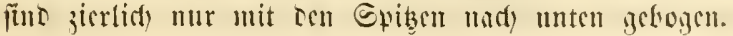




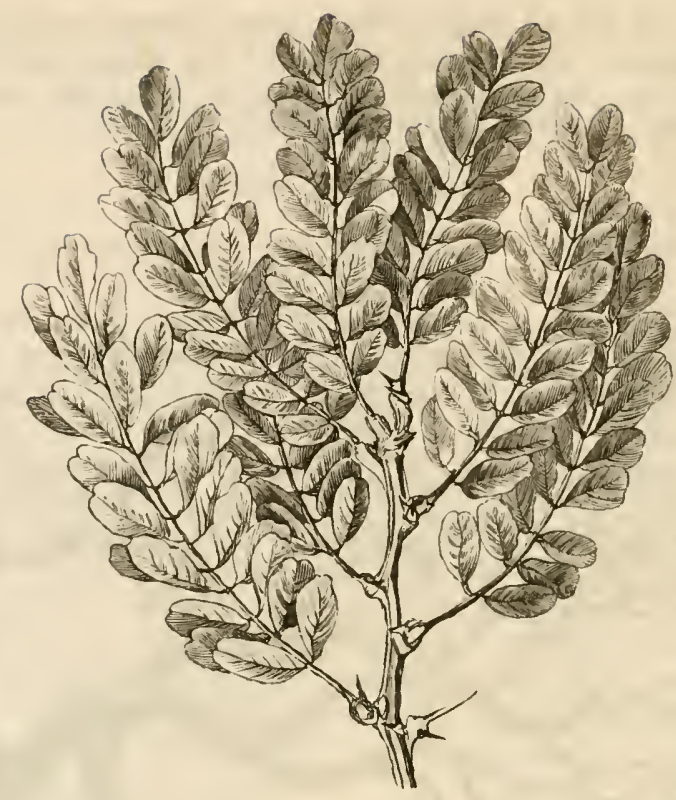

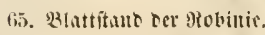

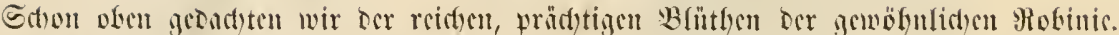

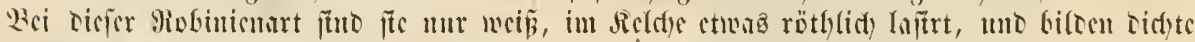
sriutuelt.

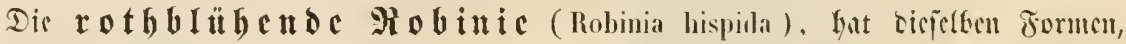

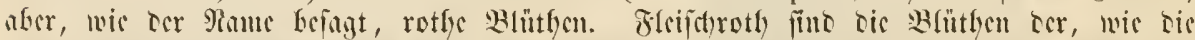

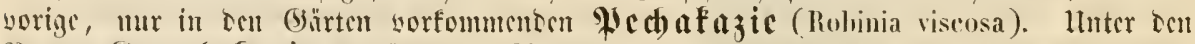

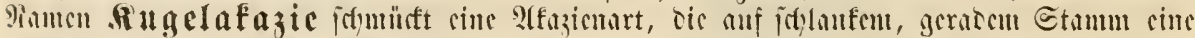

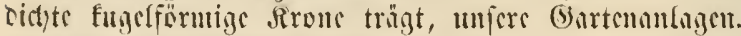

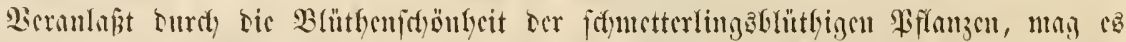

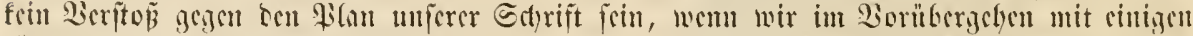

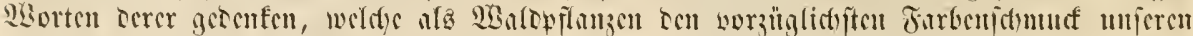
fonft blumenarmen norbijd)en Sisaloungen gebon.

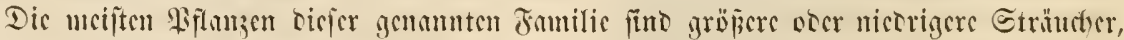

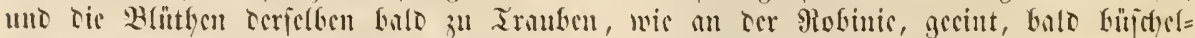

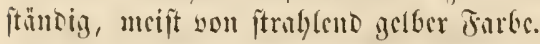

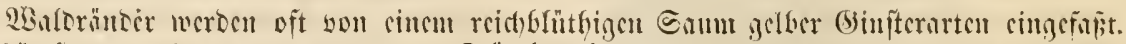

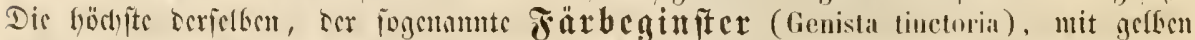

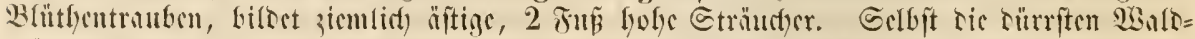

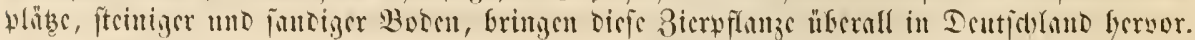

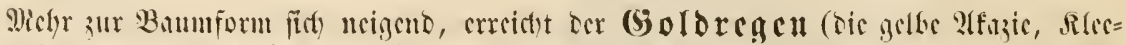

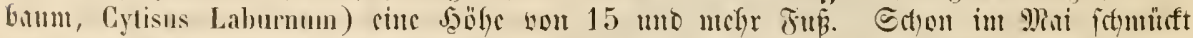

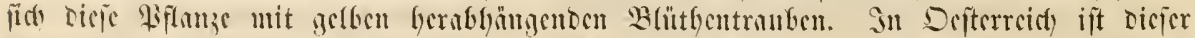

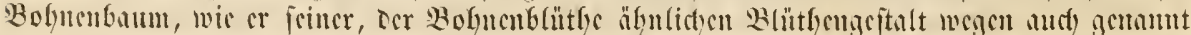

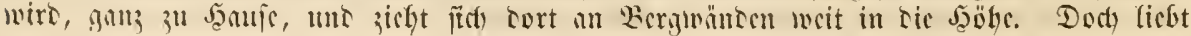




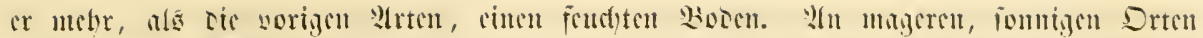

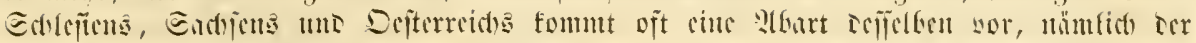

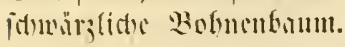

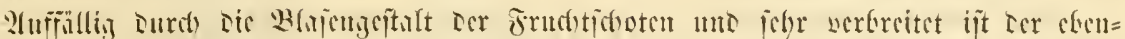

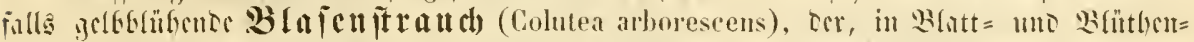

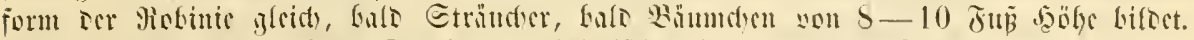

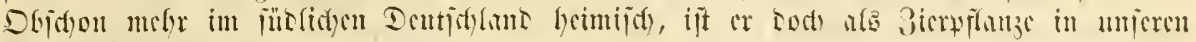
(5)irtem jibr befumit.

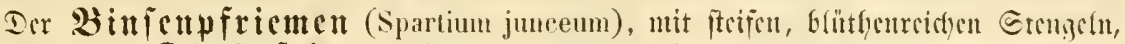

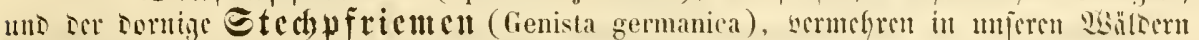

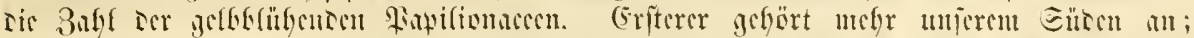

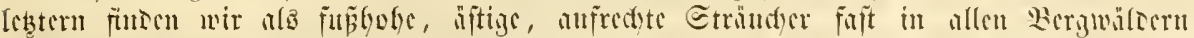
Deutid)lants.

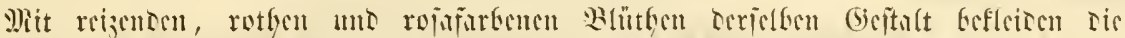
Saubchelarten (Ononis spinosa un 0. repens), mitere ats 2 Juj bofor Etrund),

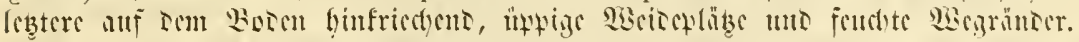

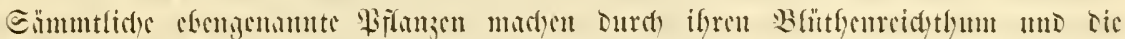

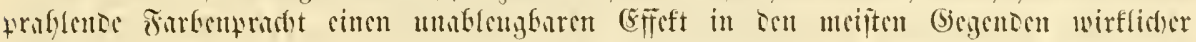
Şoljlinter.

\title{
Dite $\mathfrak{E} \mathfrak{E} \mathfrak{b} \mathfrak{x} \mathfrak{e}$
}

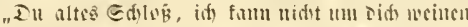

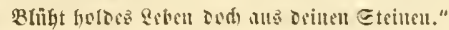

(5. (i) ciliel.

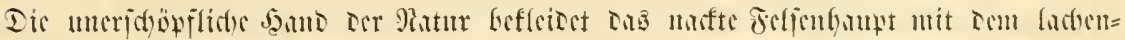

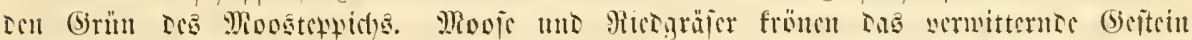

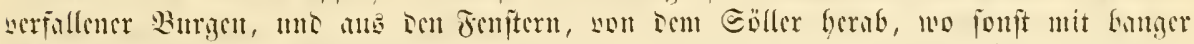

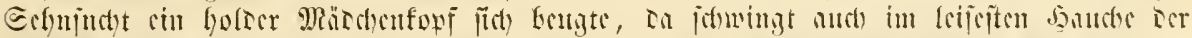

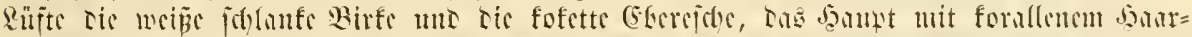

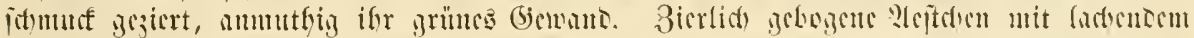

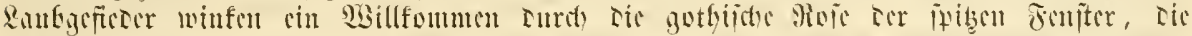

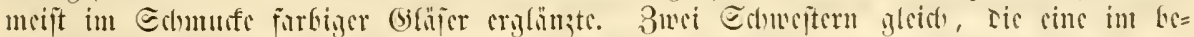

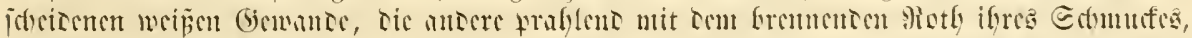

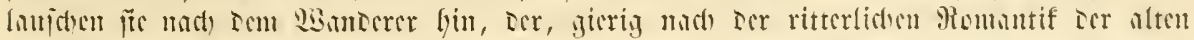

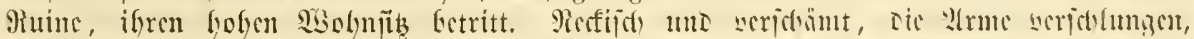

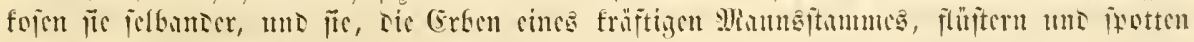

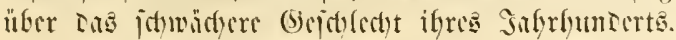

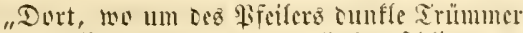 \\ Traulich fintipterns fich Der (Ephelt ishlingt,"}

(M) Battion.)

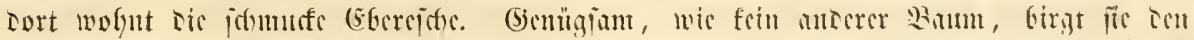

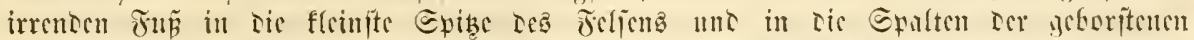

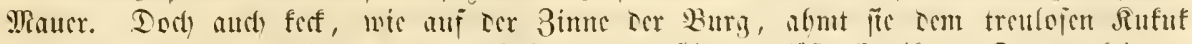

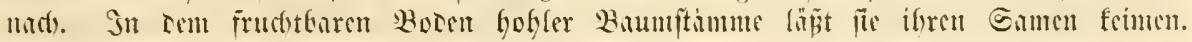




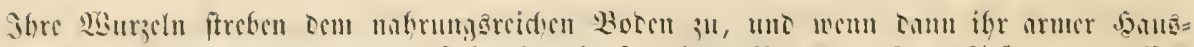

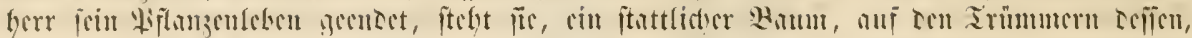
ror mcift ifor an Edönfucit jlids).

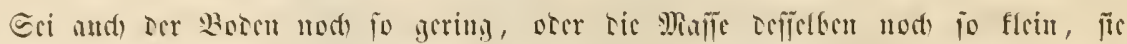

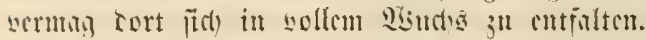

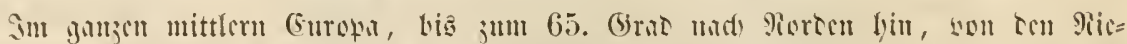

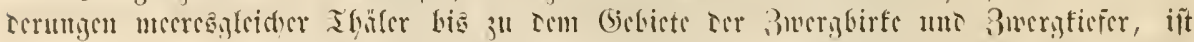

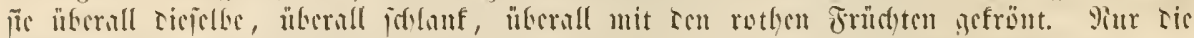

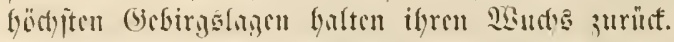

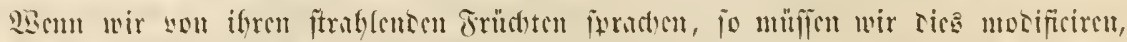

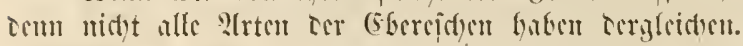

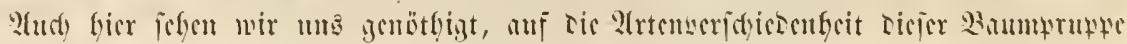
nïfor cilljugebett.

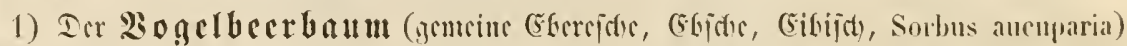

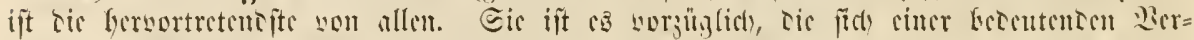

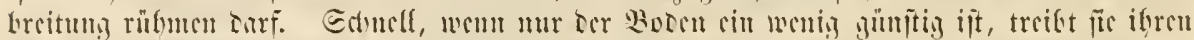

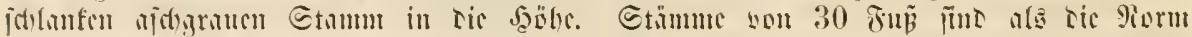

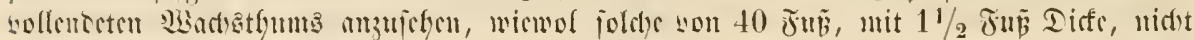
jitten jïnt.

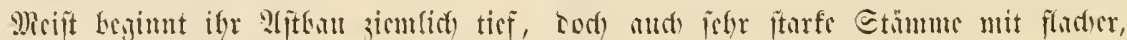

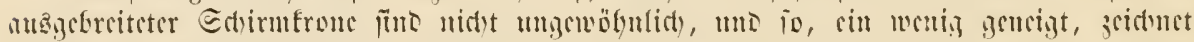

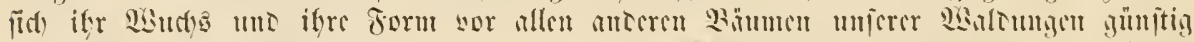

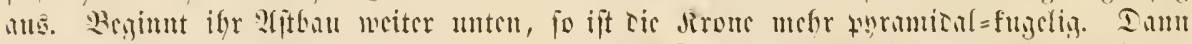

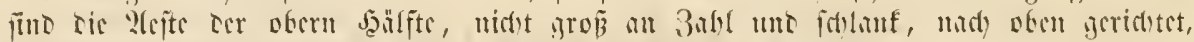
uns Dic unteren meifon nad) tor müterlidyen (Eres bin.

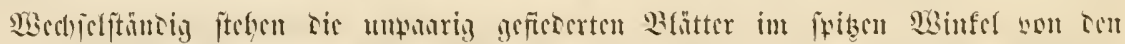

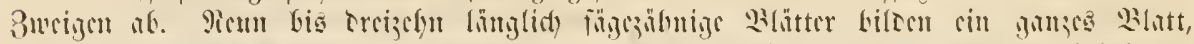

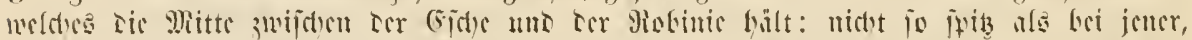

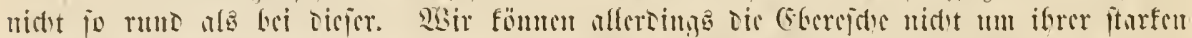

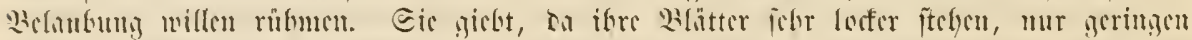

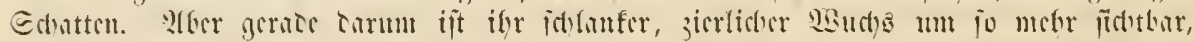

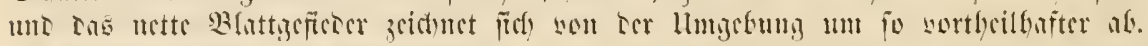

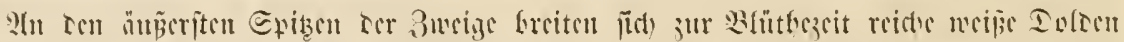

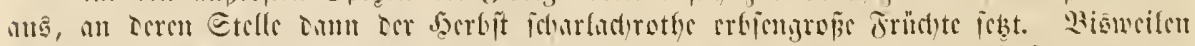

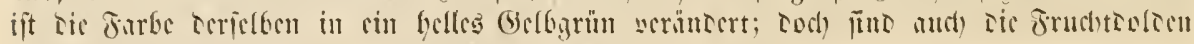

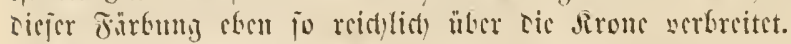

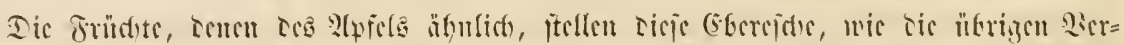

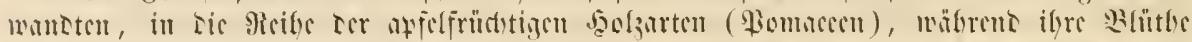

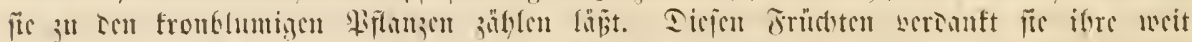

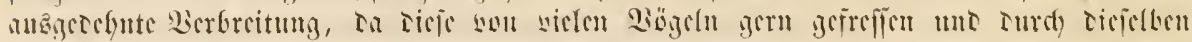
bibrall bin seridslefpt weroen.

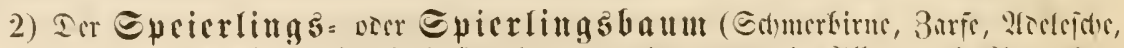

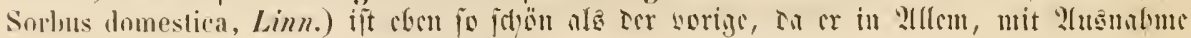
ter Früdte, ibm gleidyt.

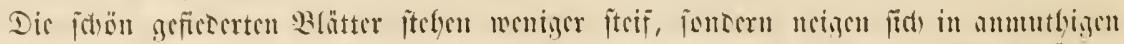

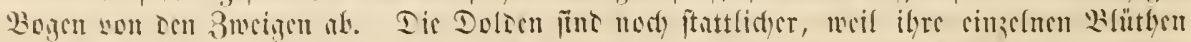

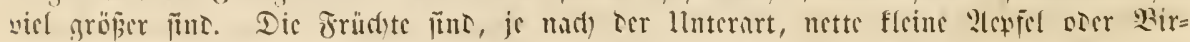

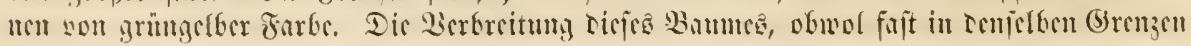




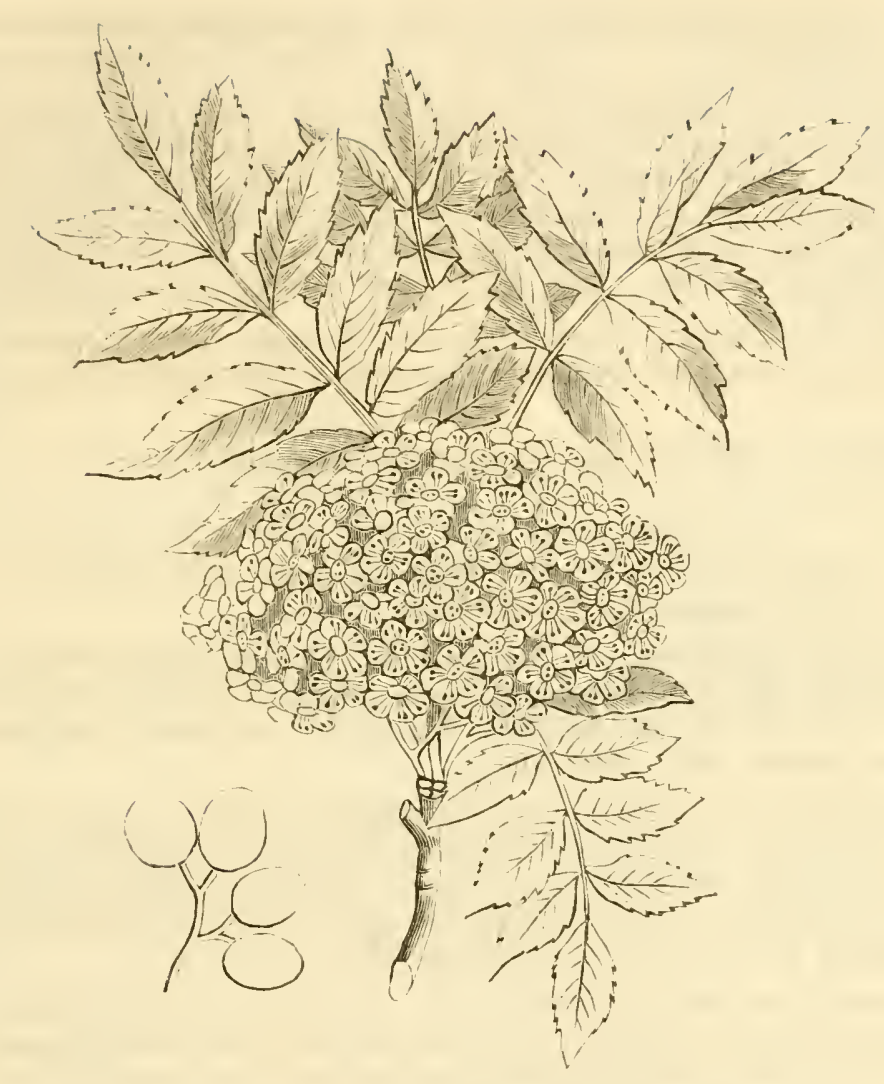

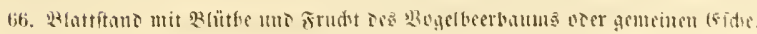

(ङ. norfergehente Ecite.)

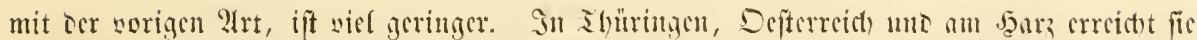

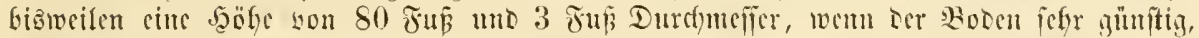

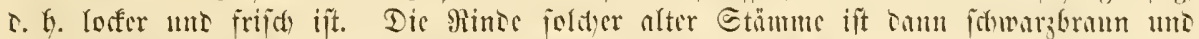
itarf aupgerifĩen. Ltu ticic böbs zu erreicten, besarf fie mebr als 100 bis 140 sabre,

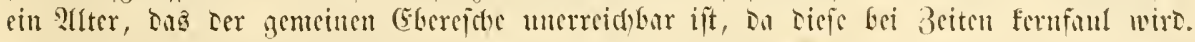

3) Die halbgefieberte (Eberefde (Sorbus hybrida). Shre wlïter fint nicht

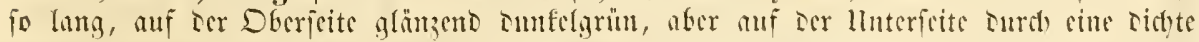

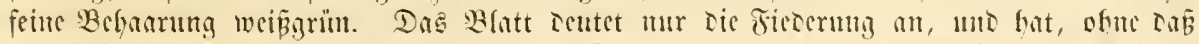

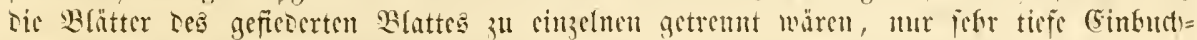
tungen, wie umier (Eirbenblatt. Dabei ift ter Rant leidst gezäbut.

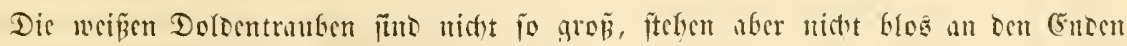

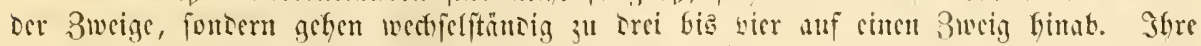
rumelidyen Jrüthte gleidyen tenen unter 1. beforiebenen, werden aber bei fer Reife Graunroth.

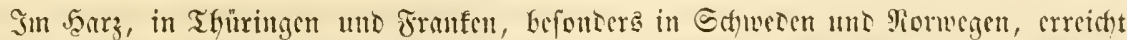

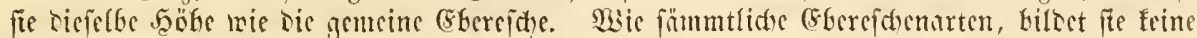
felfititänsigen WBifter, fontern fommt meipt sercimgelt wor, auper wo ticie bänte ourds 


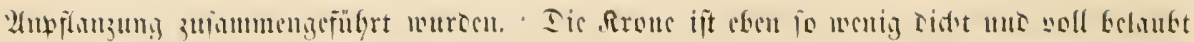
al cic icr criten ?Irt.

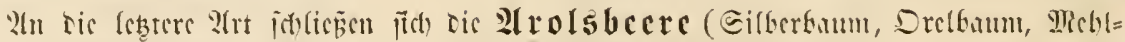

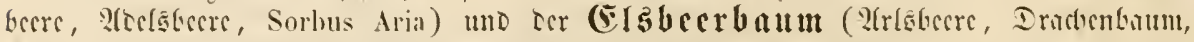

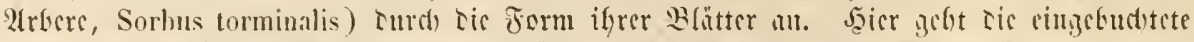

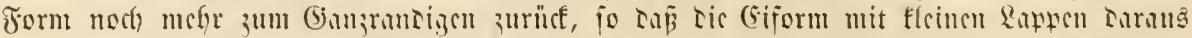

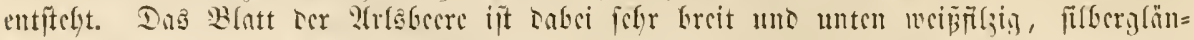

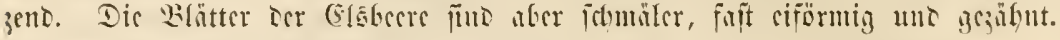

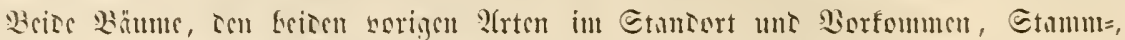

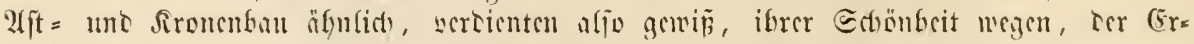
wifonumg.

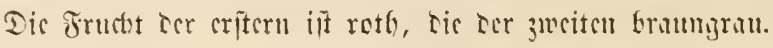

Da wir eimmal bei sor Fimilis Ier Fonacen fteben, fo betracten wir noch sic

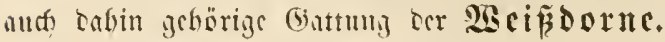

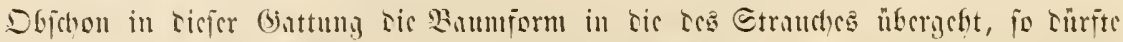

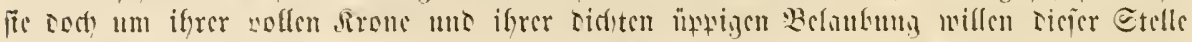

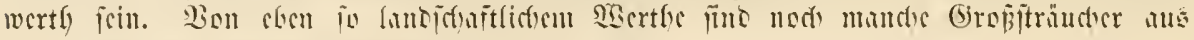

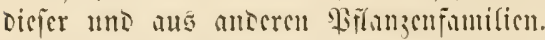

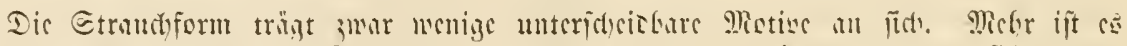

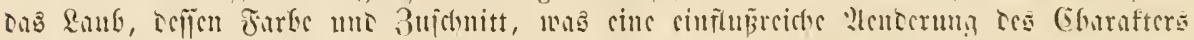

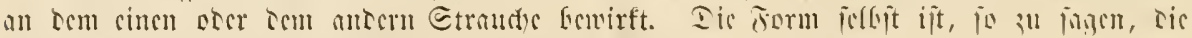

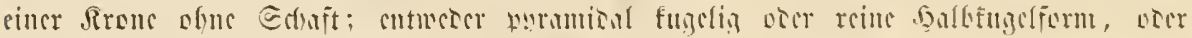

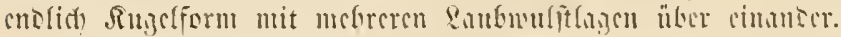

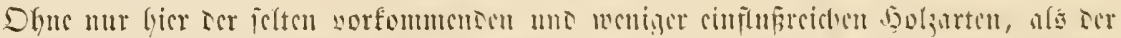

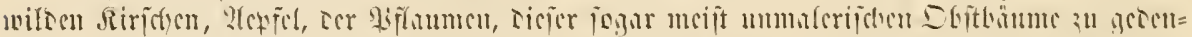

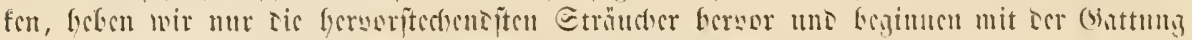

\section{cer 2iseiźdorue ( Crataegus).}

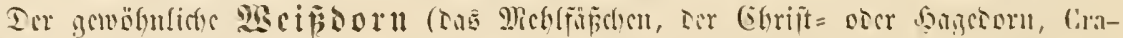

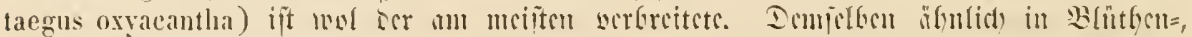

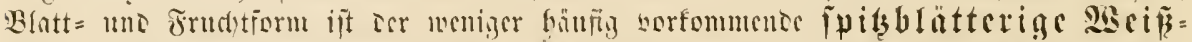

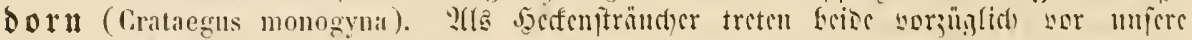

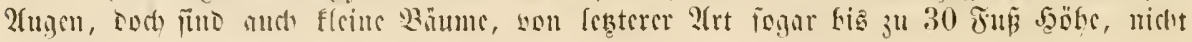
3ll islten.

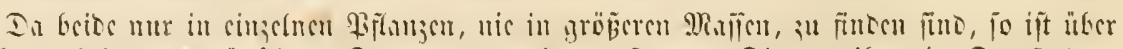

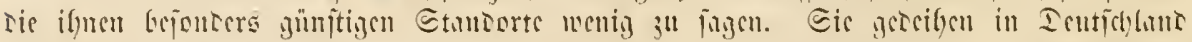

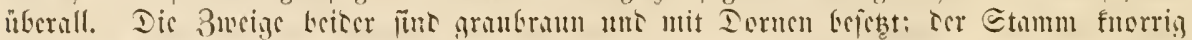
uno viclfist) grtiongen.

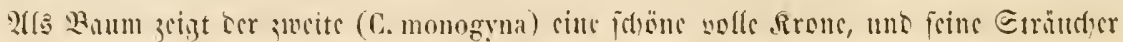

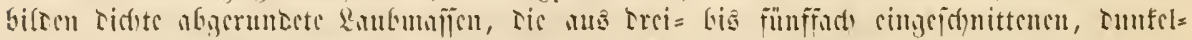

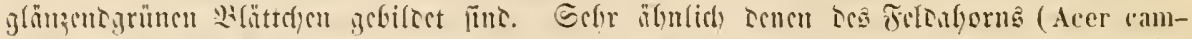

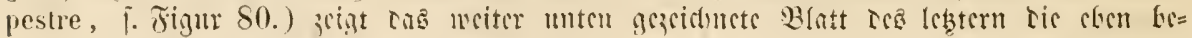
idiricue Bistatiorm.

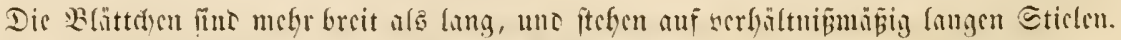

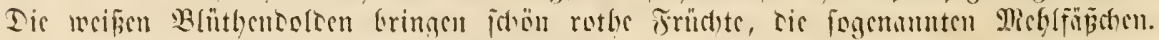




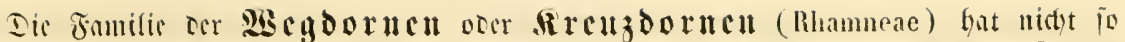

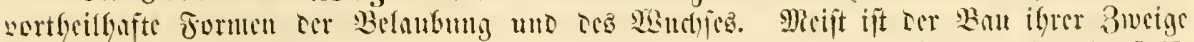

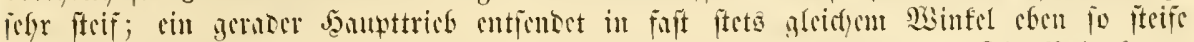

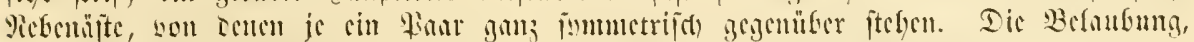

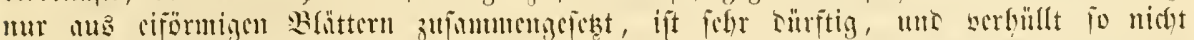

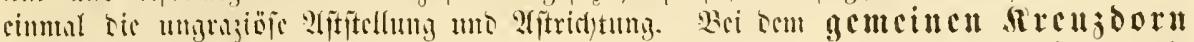

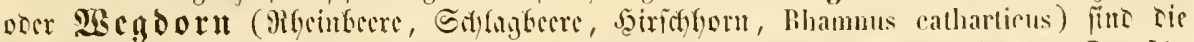

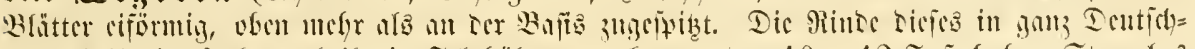

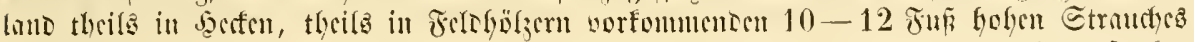

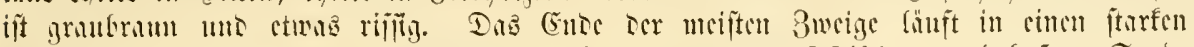

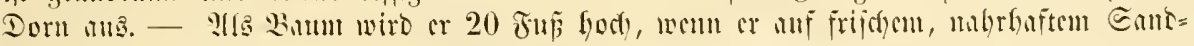
bocen itiht.

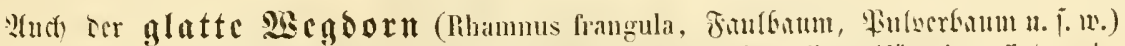

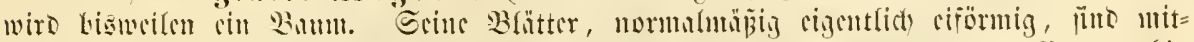

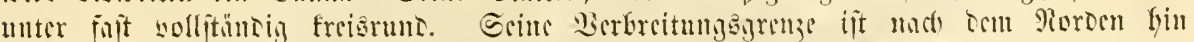

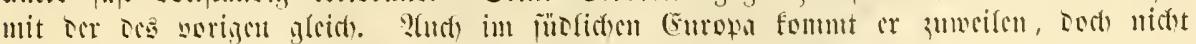

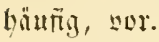

Dic Mfpen be

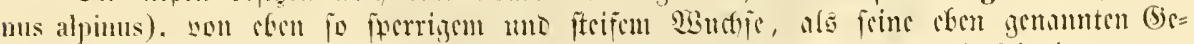

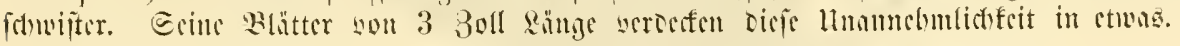

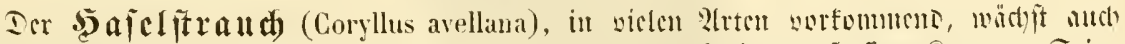

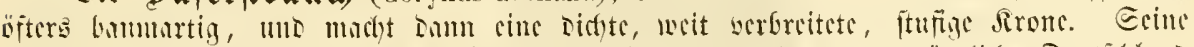

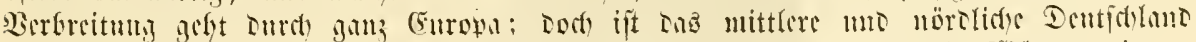

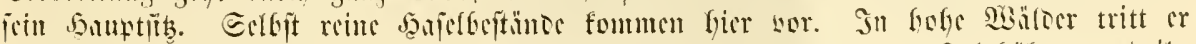

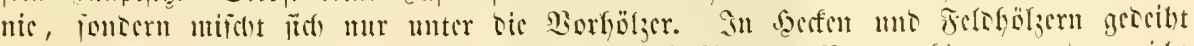

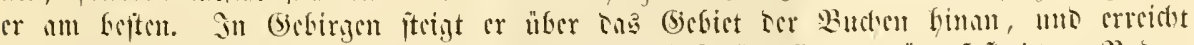

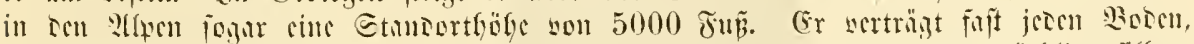

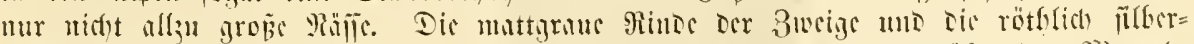

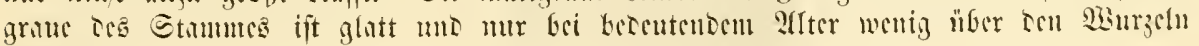
aufycriाা

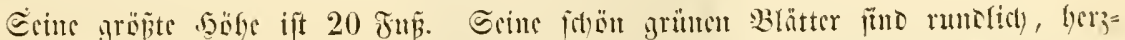

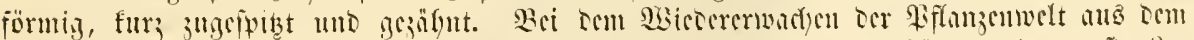

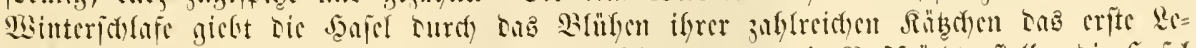

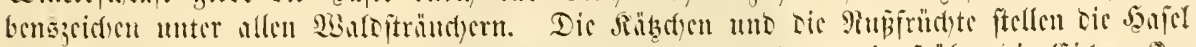

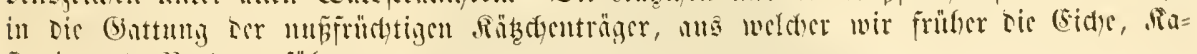

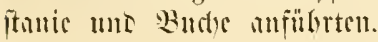

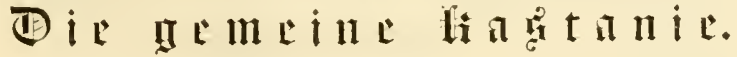

\author{
[Moǵtaftanic.]
}

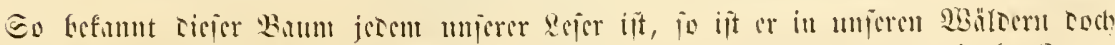

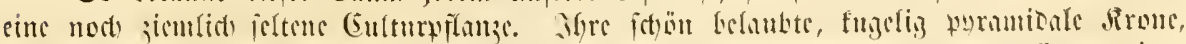

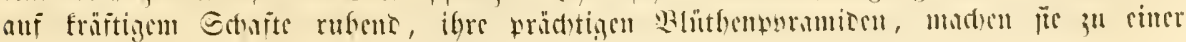




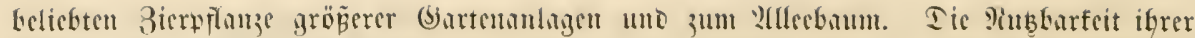

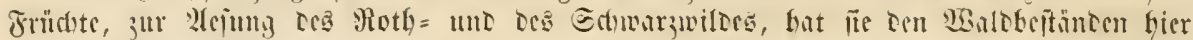
une ta Ecigemijott.

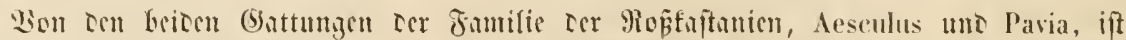

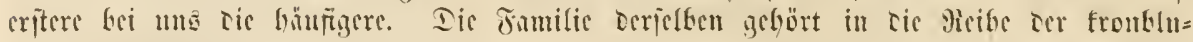
migen Soljtirlutar.

1) Dir gemciuc fiaftanic (Ropitnitunic, Aesculus hippocastanum, Limn.) muroo

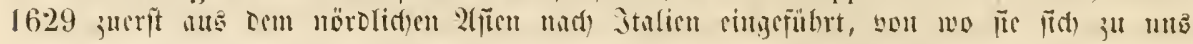

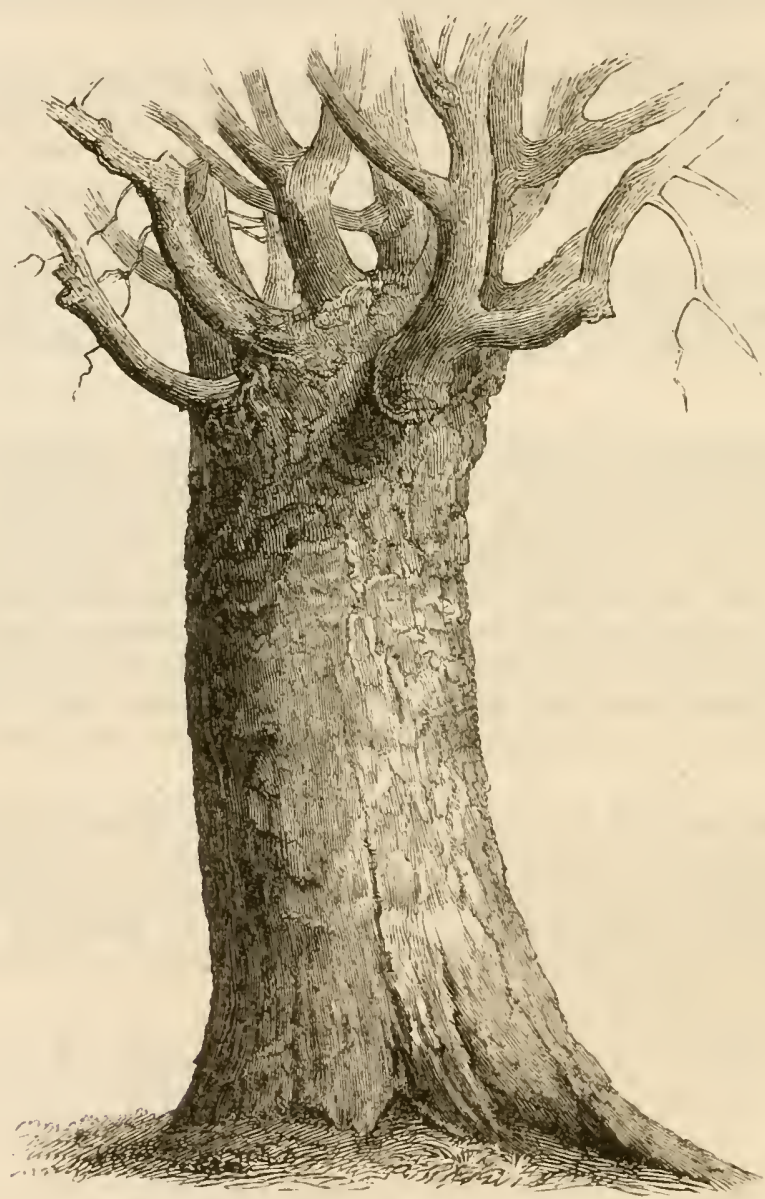

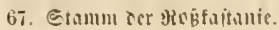

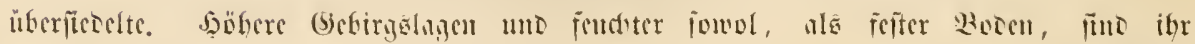
jumicer.

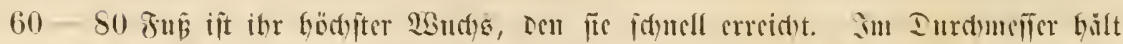

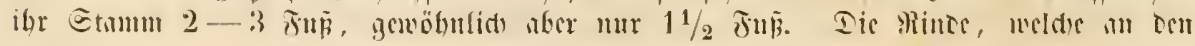




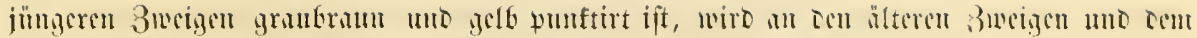

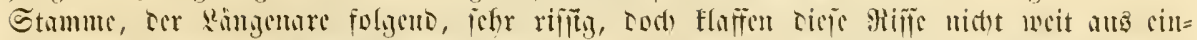

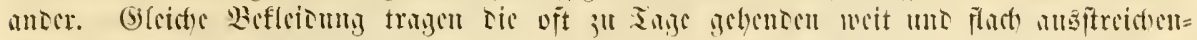
Den 2 burjefln.

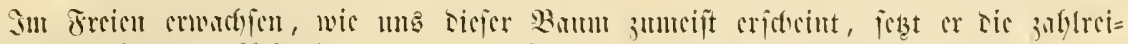

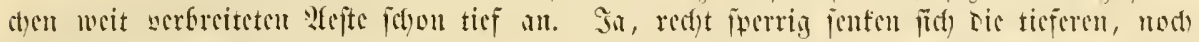

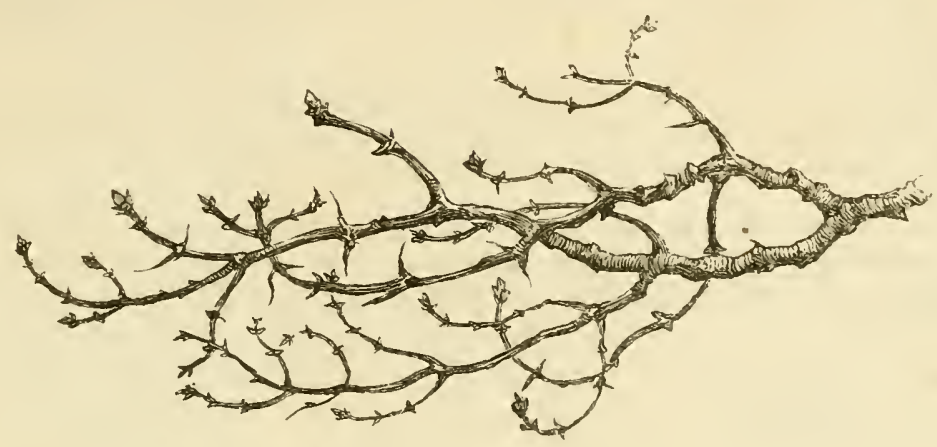

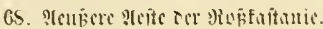

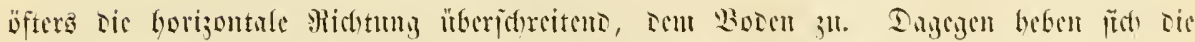

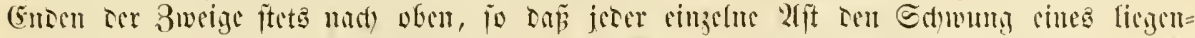

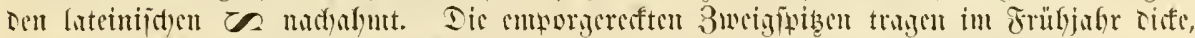

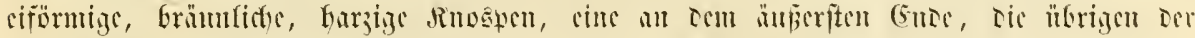

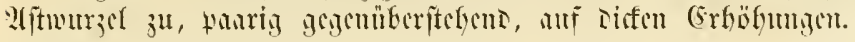

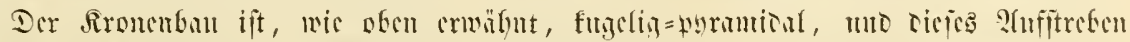

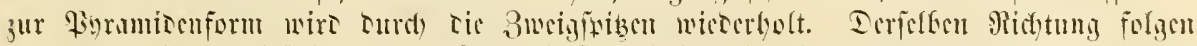

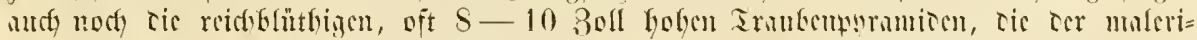

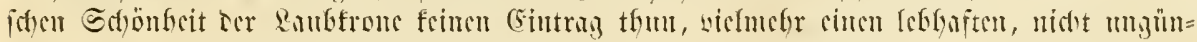

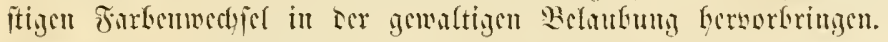

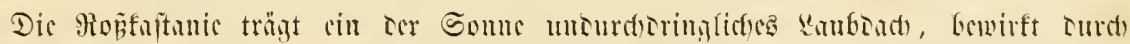

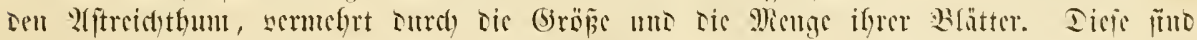

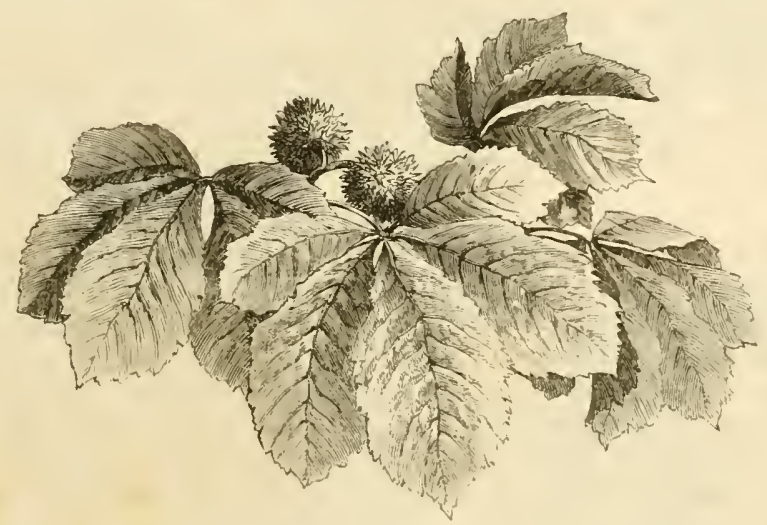

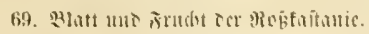




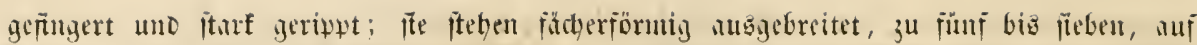

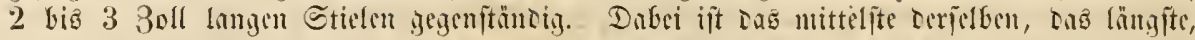

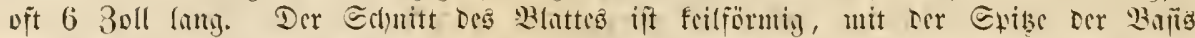

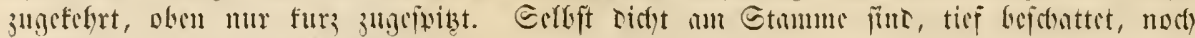
reichlid) mit Blätern bejebte Sriebs.

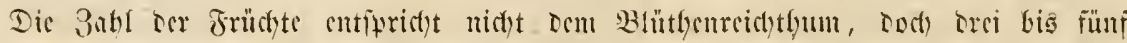

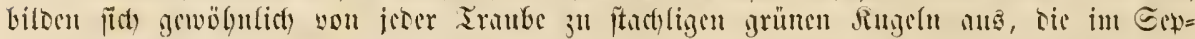

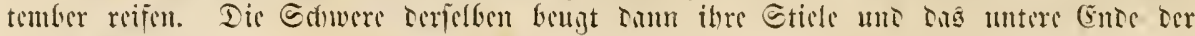
Buscize berub.

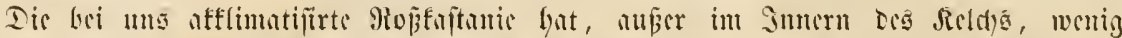

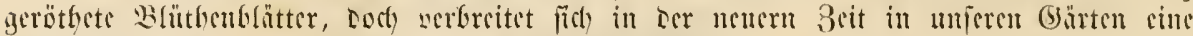

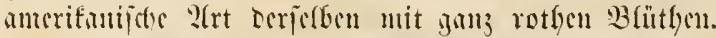

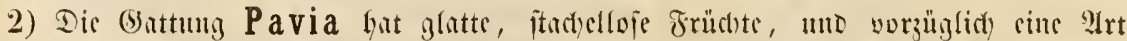

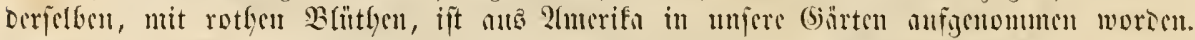

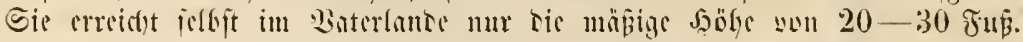

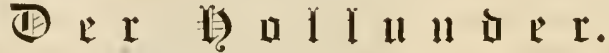

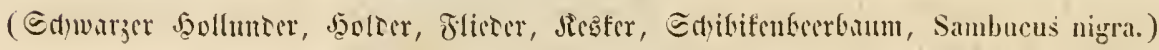

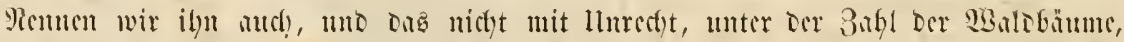

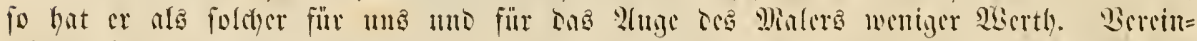

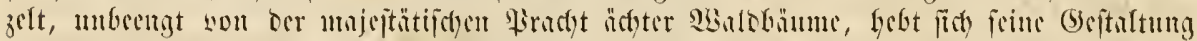

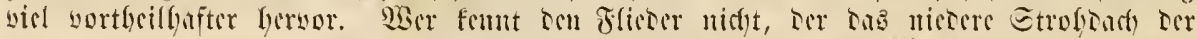

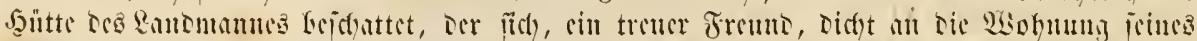

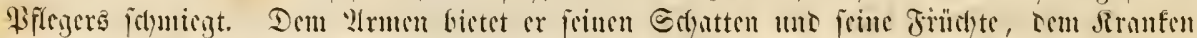

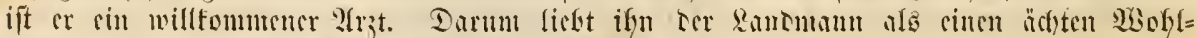

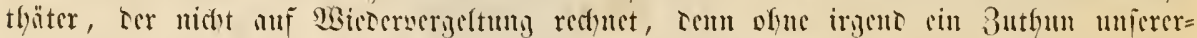

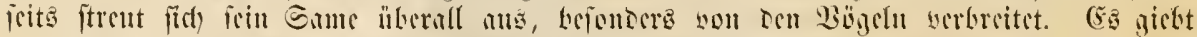

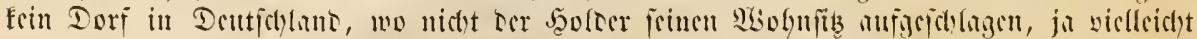

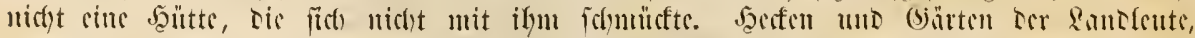

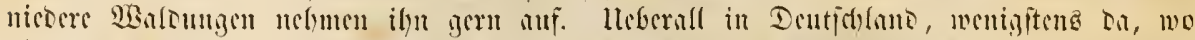

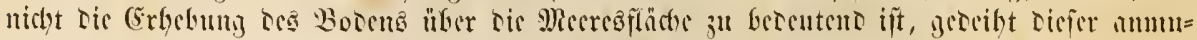
thitige 2 Bamt.

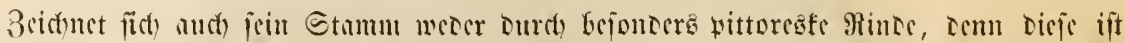

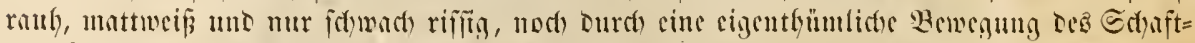

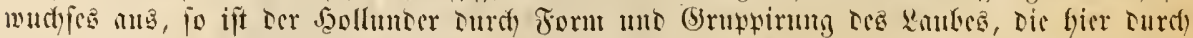

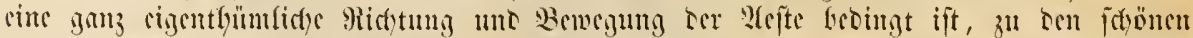

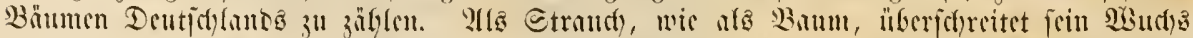

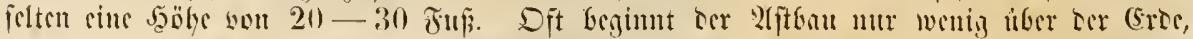

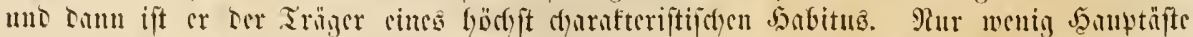

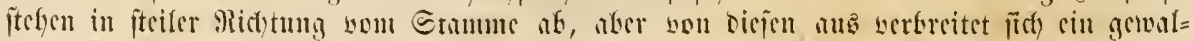

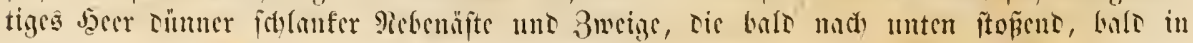




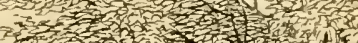

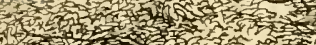

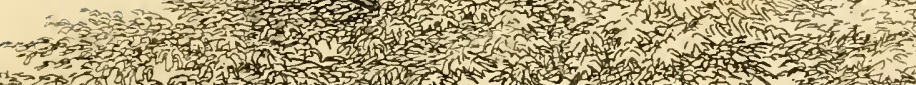

$\begin{array}{llll} & \end{array}$

Mr

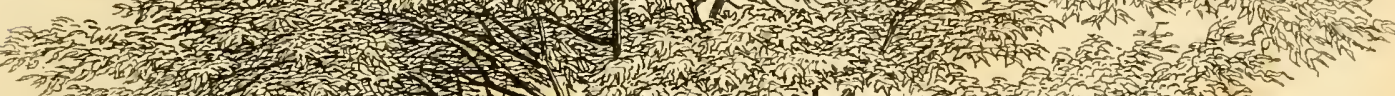

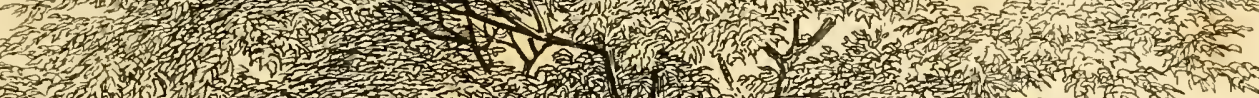
3.

隹

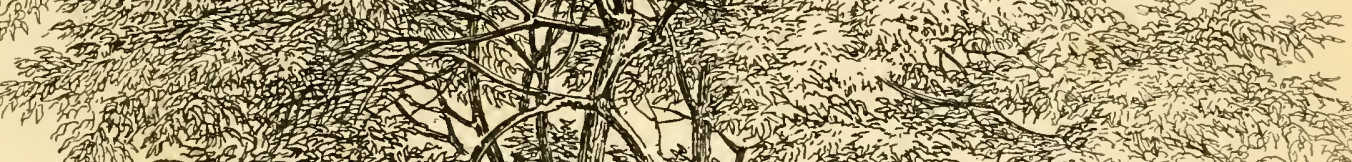

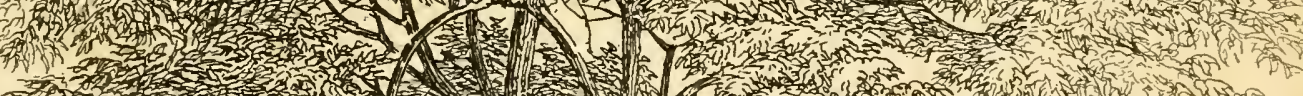

(1)

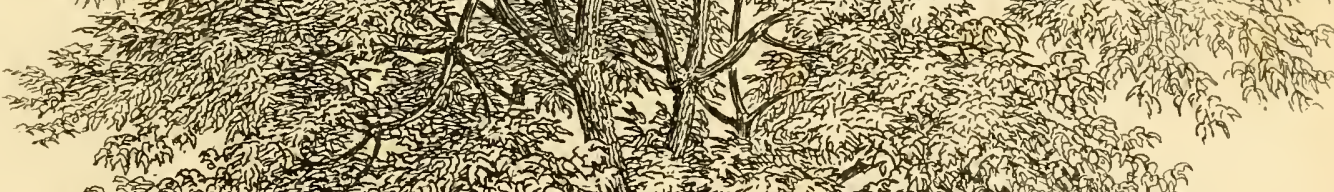

197,

-

$\rightarrow-2$.

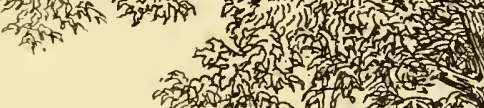

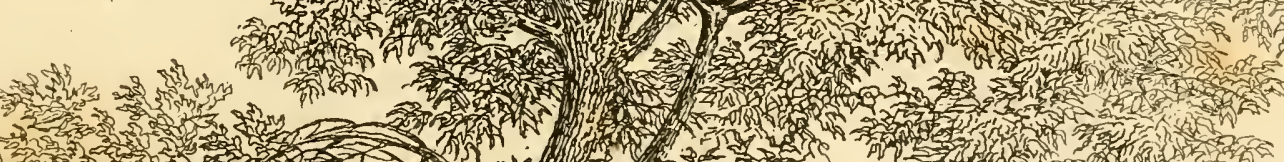

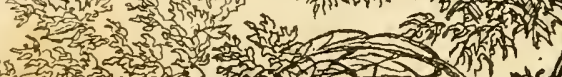

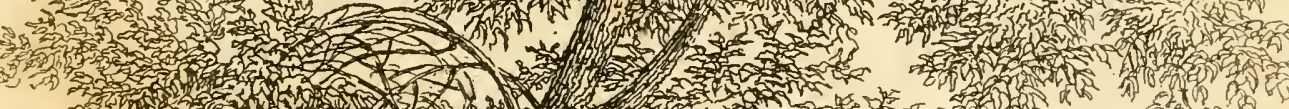

3.

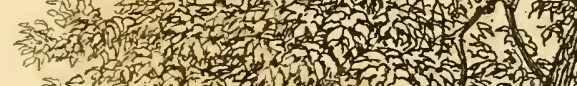

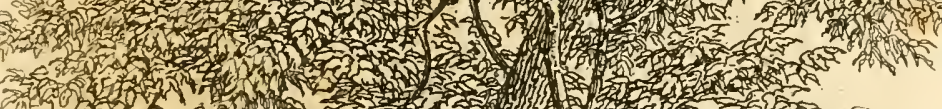

$3(3)$

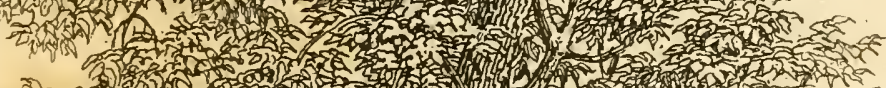

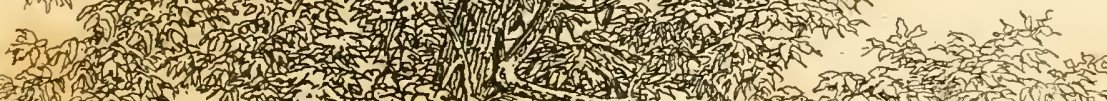

\%

14

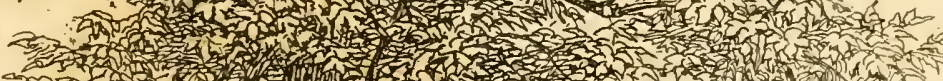

3.

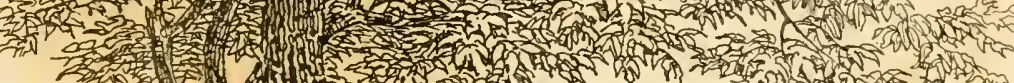

2-5.

2.

(1) 



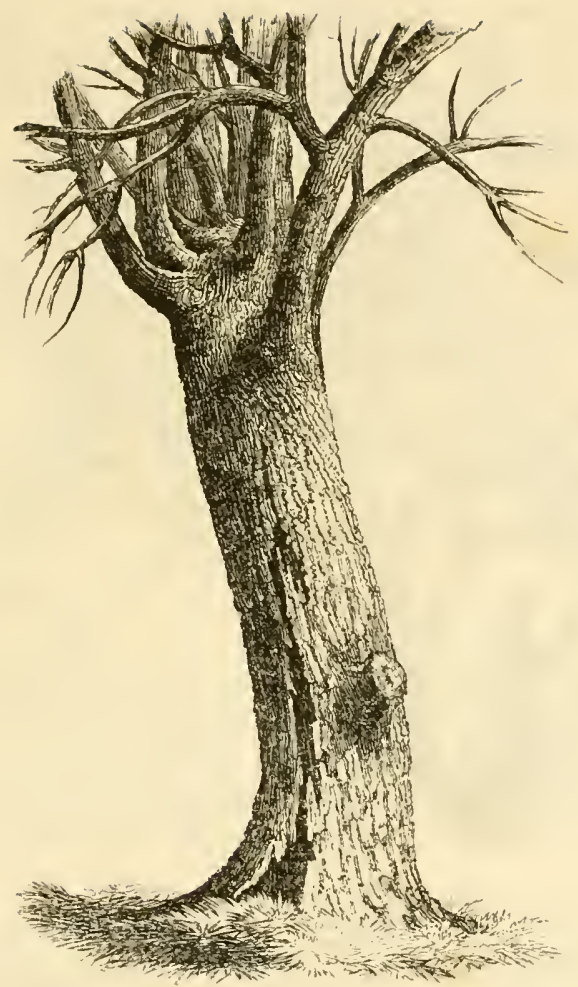

70. Etmm tes sullumbers.

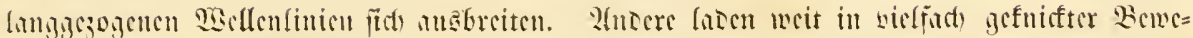

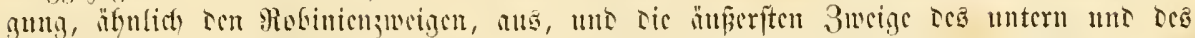

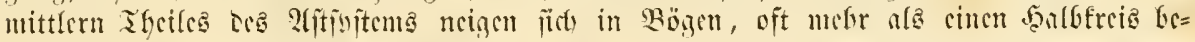

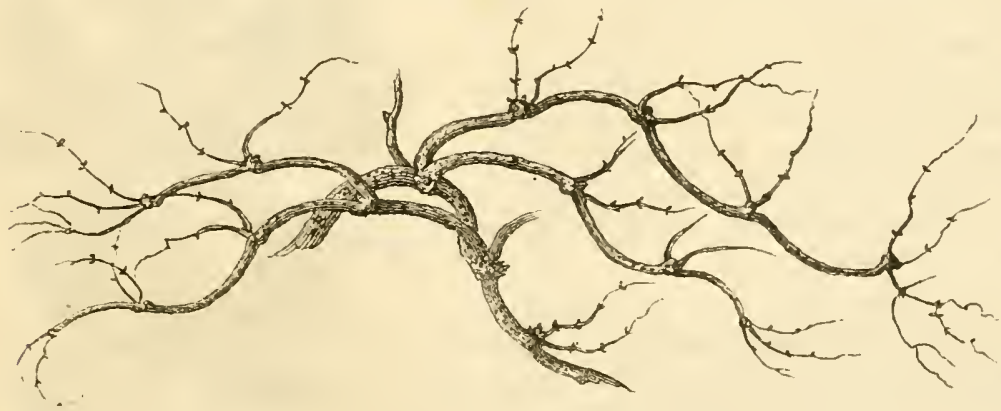

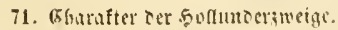

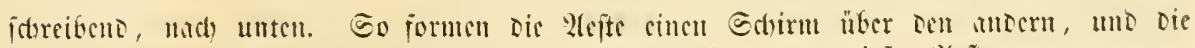

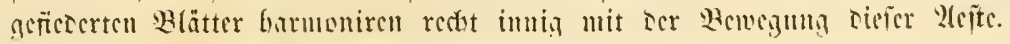




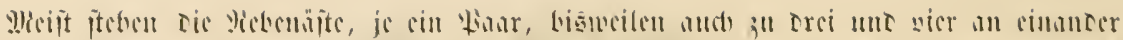

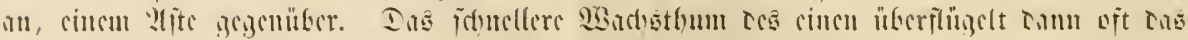

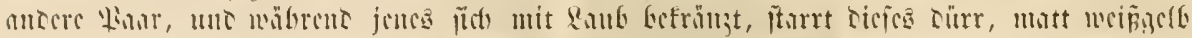

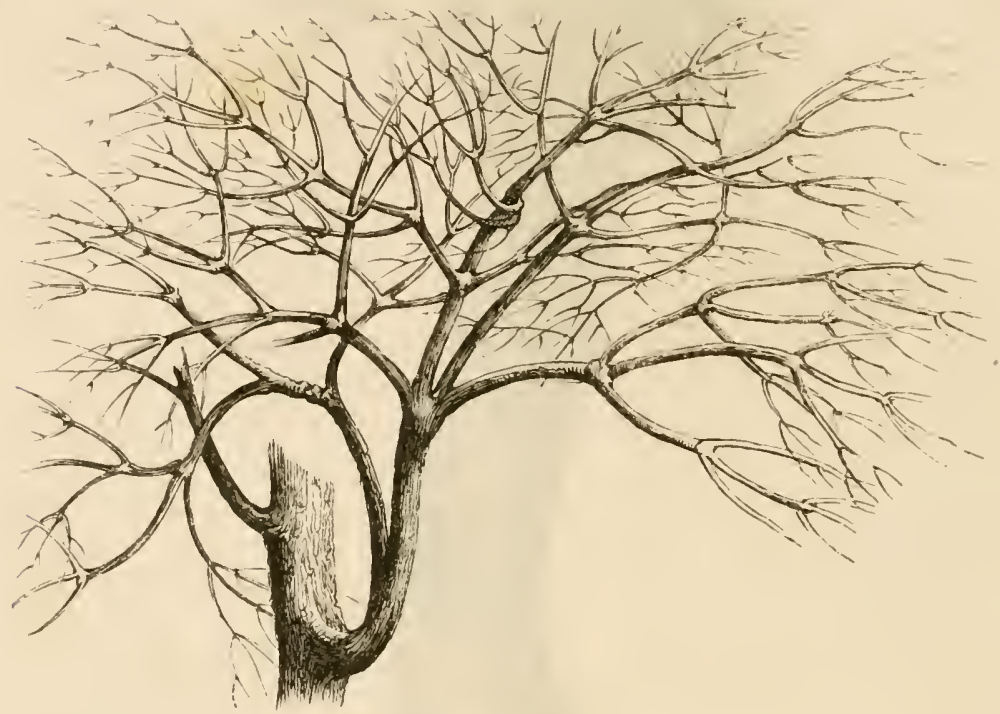

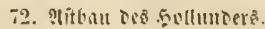

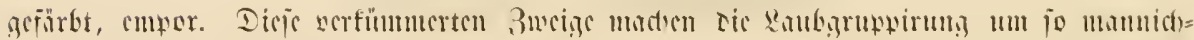

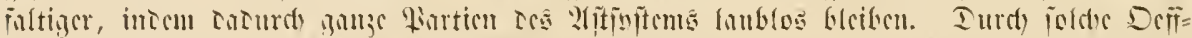

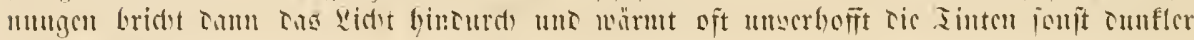
sunfurticn.

Iic Bäter (erciten fict) entureter borizontal

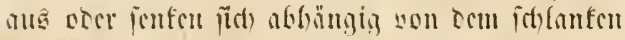

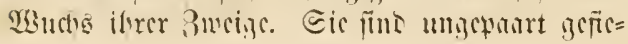
rert, ans fünf bis firben cifömtizen, famb

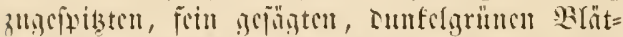
tern ;"1.

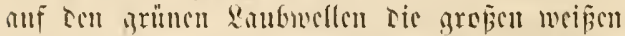
Bilutbentoform, an Eeriu Etclle im Eeptember

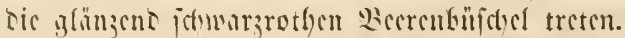

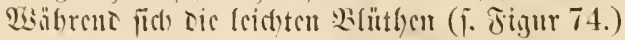
nath ofen ridten, werten ticfe won ifyer cigenen Estuere mad) unten agezojen.

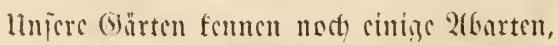
wic sen peteriflenblatterigen Sol= lunder (Sambueus laciniata), mit ticj cinge= jonittenen, fajt geriffencu Baftern. Bes Dicjer

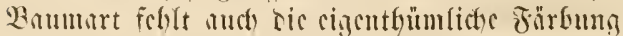
oce Hhattes, ric mir foon früber bei manden

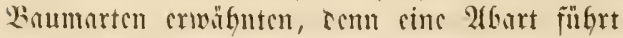
acicterfte R्Rlätter.

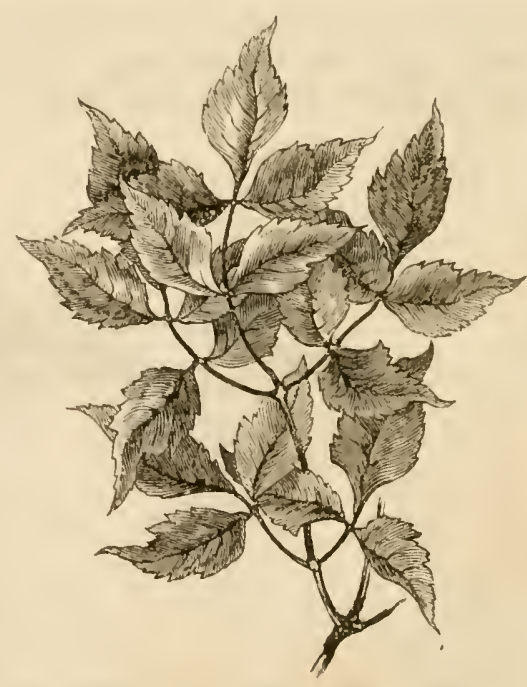

73. Blattifant tee fonthudre. 


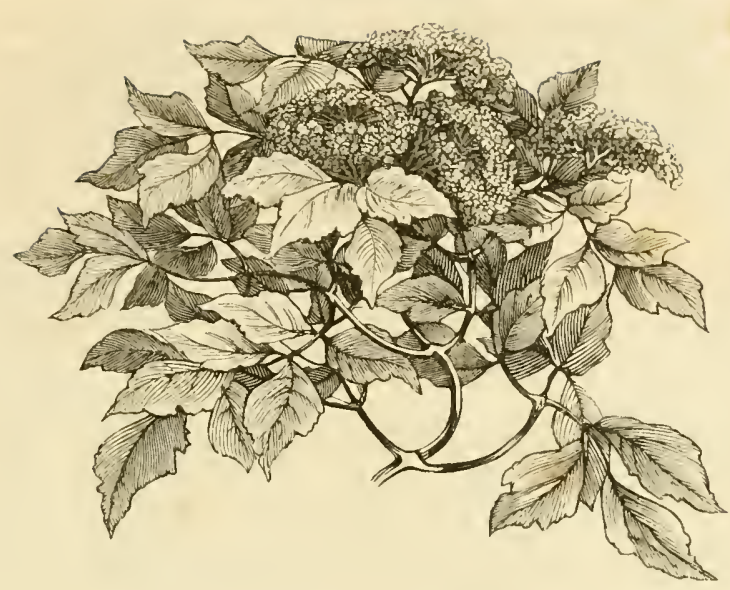

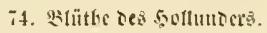

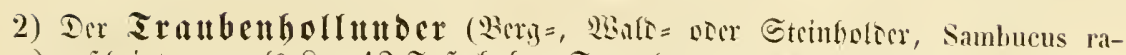

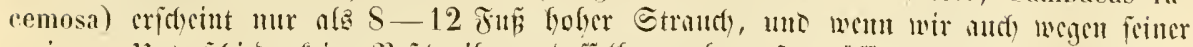

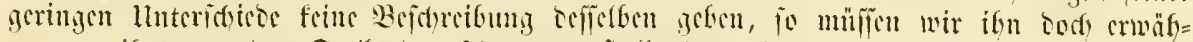

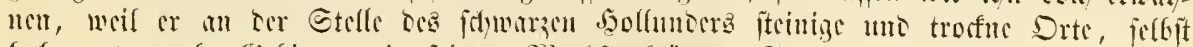

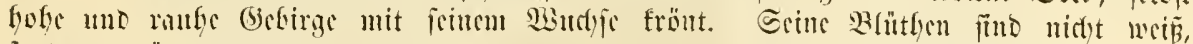
jontern grün.

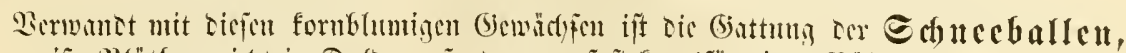

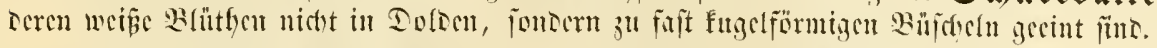

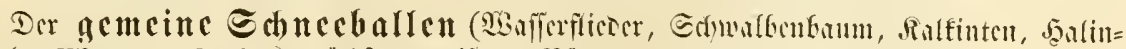

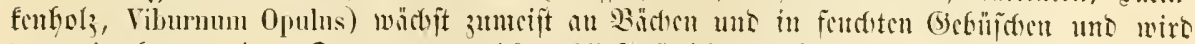

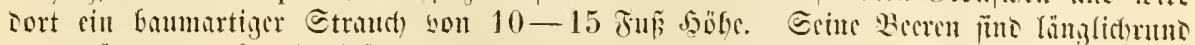

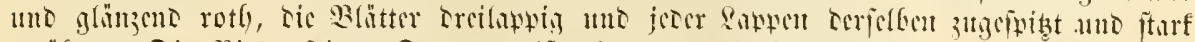

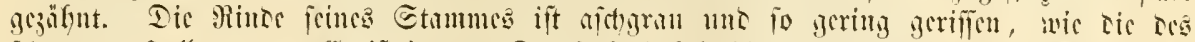

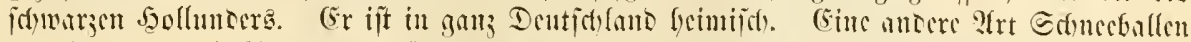

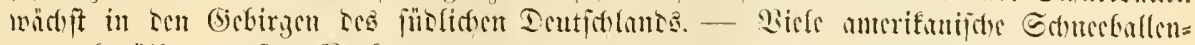
arten besölfern unifere Finfe.

\section{(1) $\begin{array}{lllllllll}\mathfrak{l} & \mathfrak{r} & \mathfrak{A} & \mathfrak{l} & \mathfrak{a} & \mathfrak{r} & 11\end{array}$}

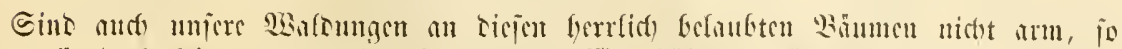

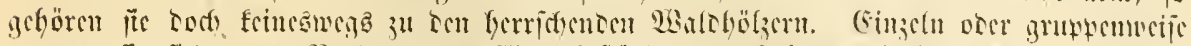

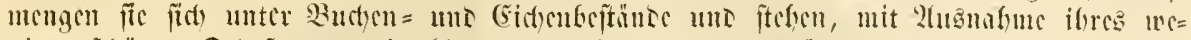

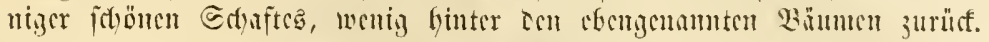

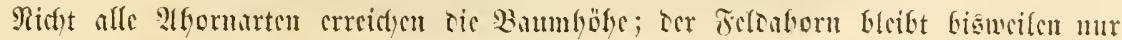

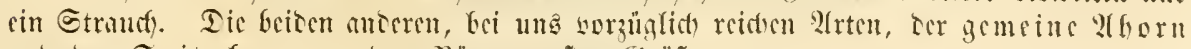

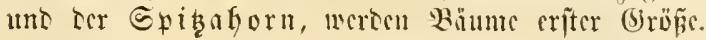


Wor alfen tentiden MEs

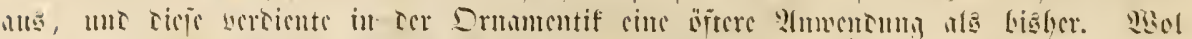

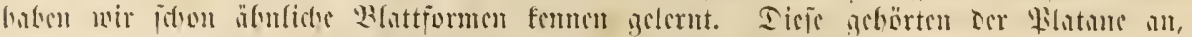

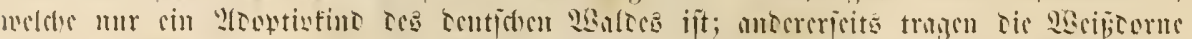

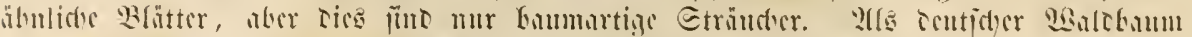

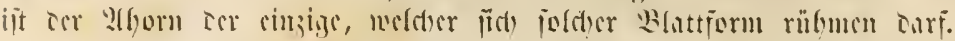

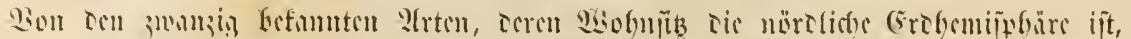

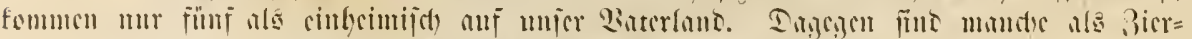

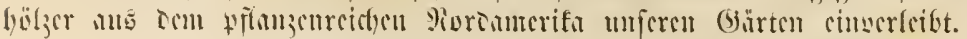

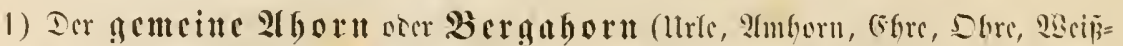

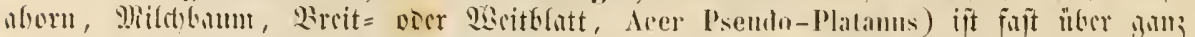

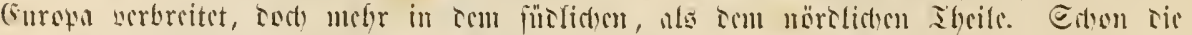

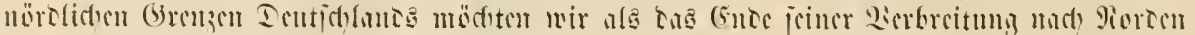

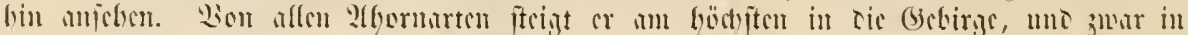

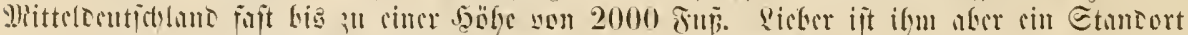

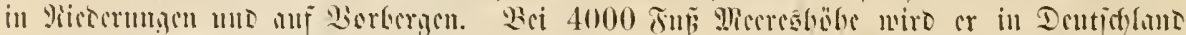

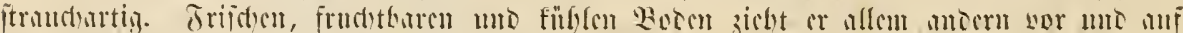

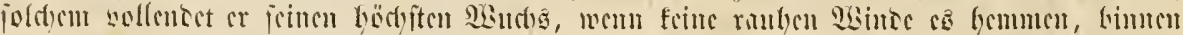

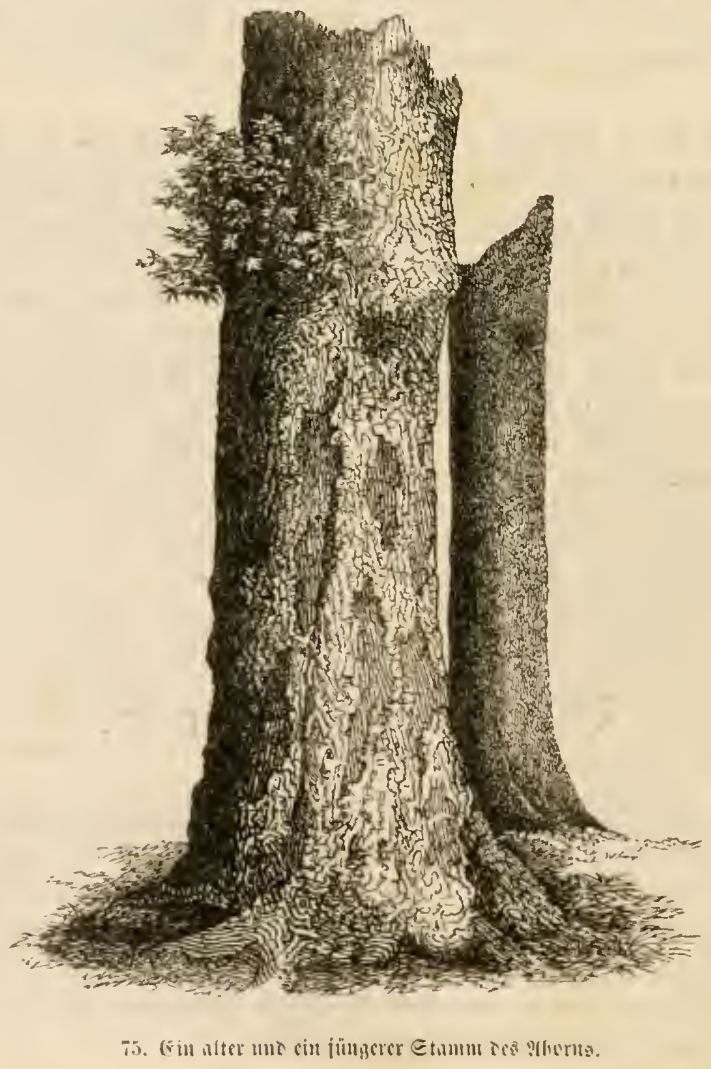




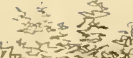

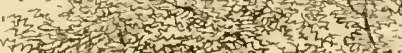

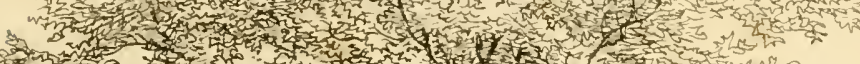

(2)

1.

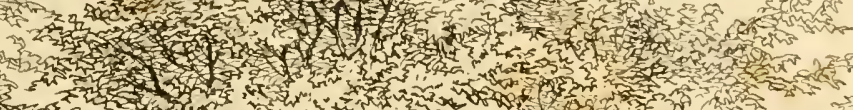

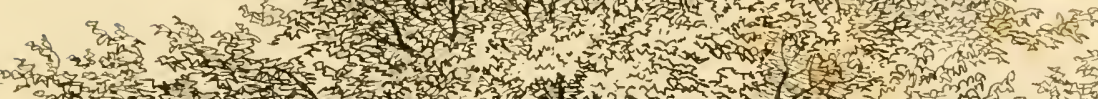

w

3.

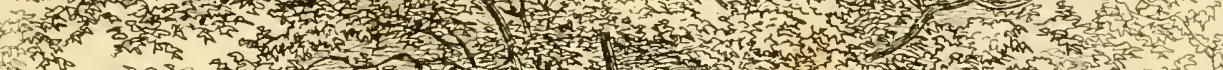

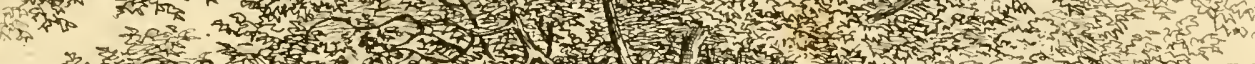

Hon

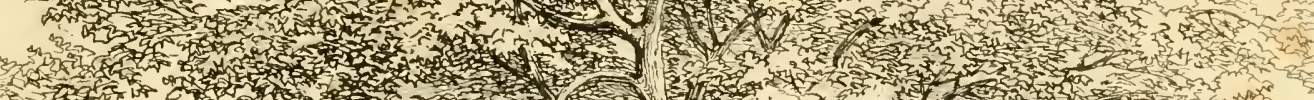

$\begin{array}{llll}2 & \end{array}$

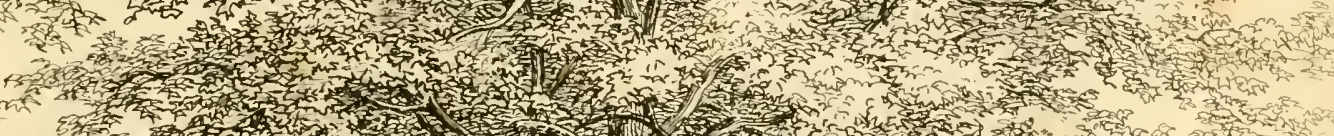

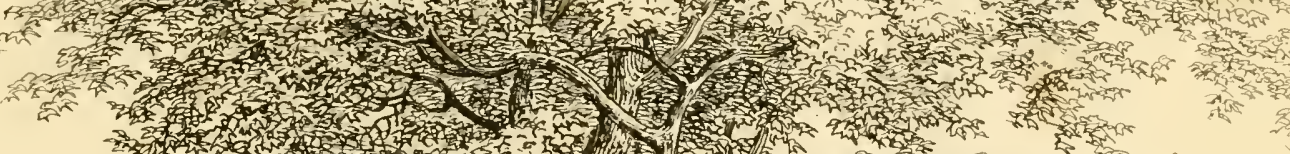

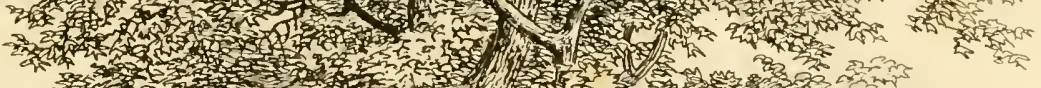

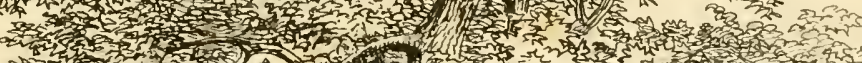

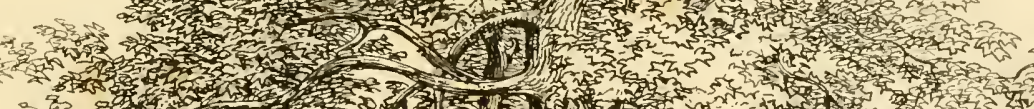

m.

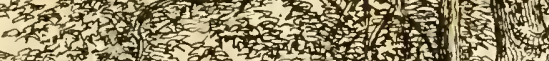

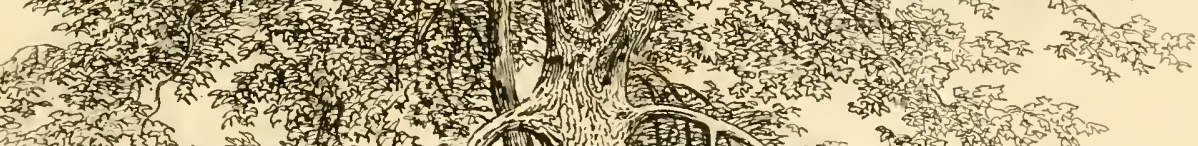

mon

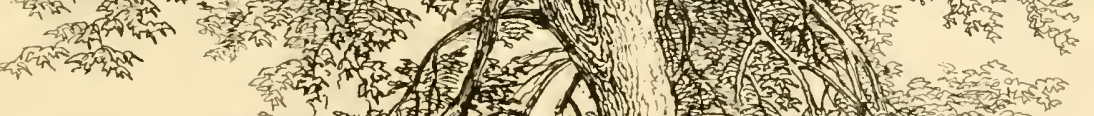

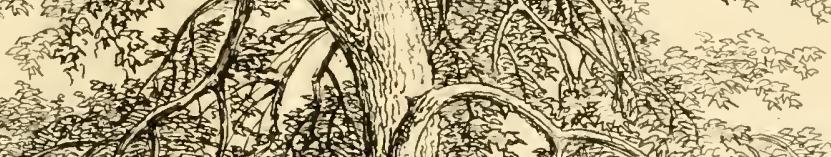

(1)

(3)

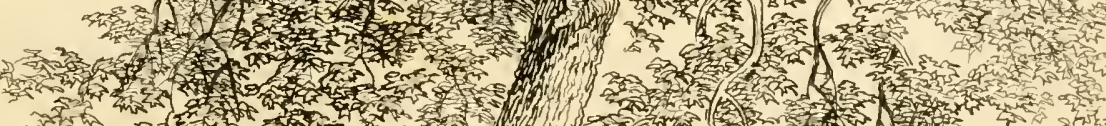

, nh

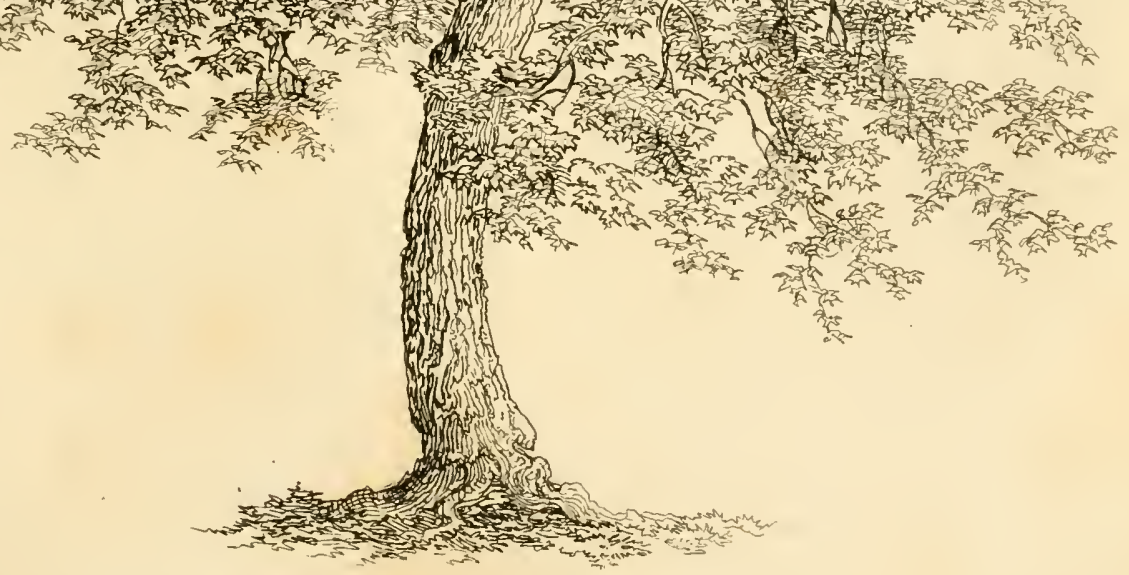

Der Aljotn 



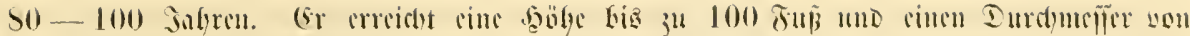

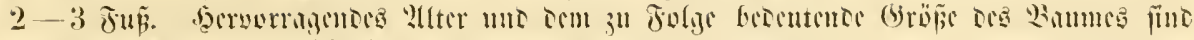

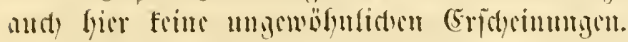

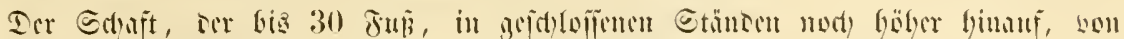

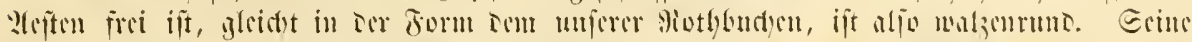

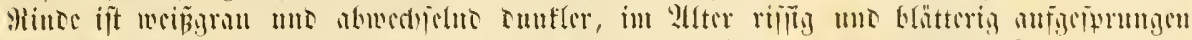

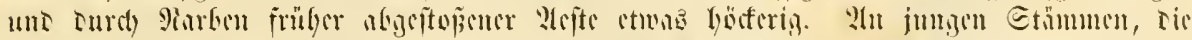

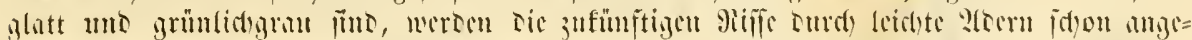

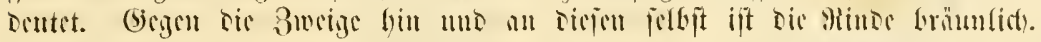

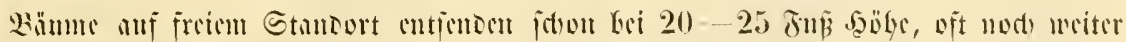

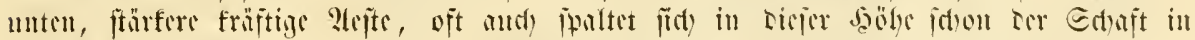

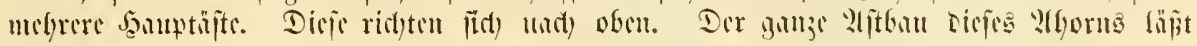

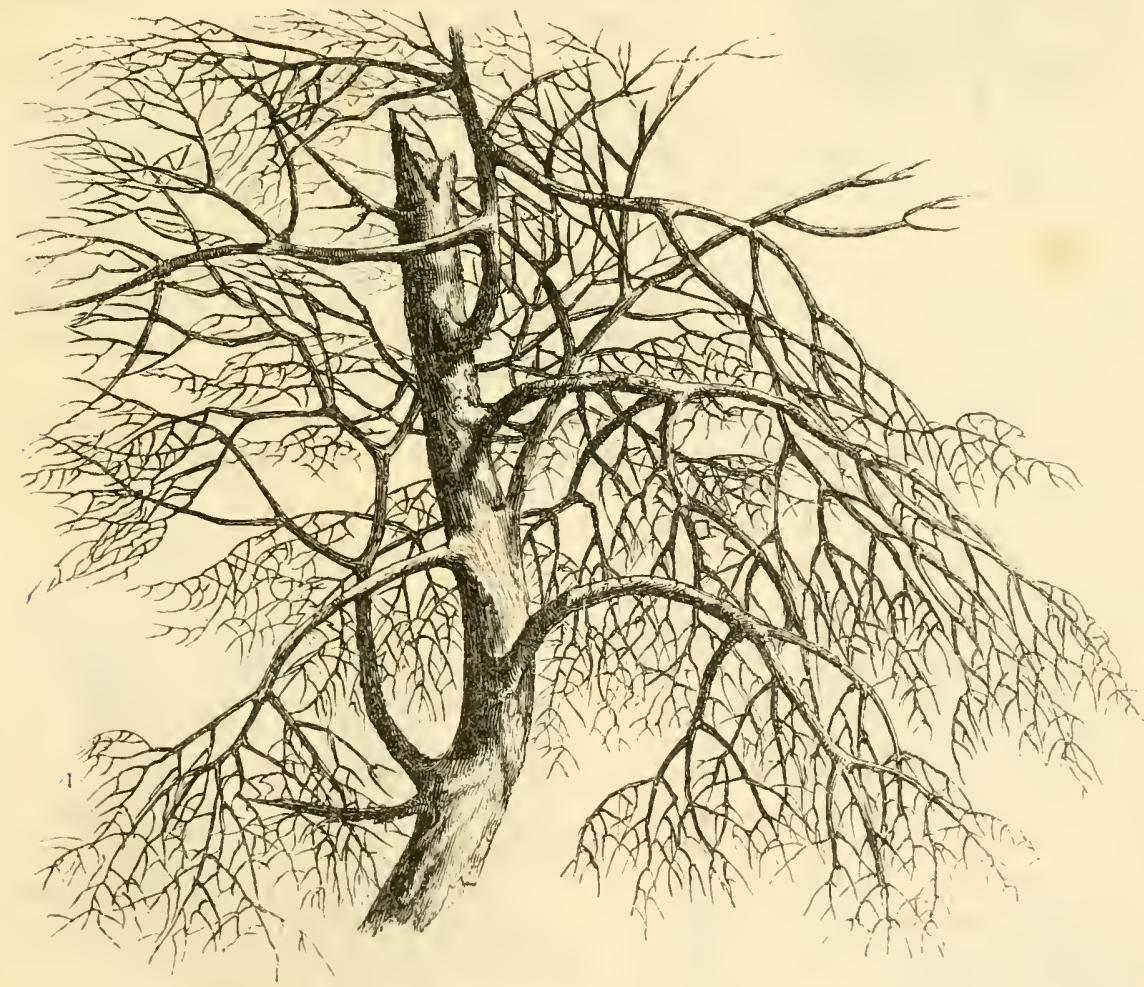

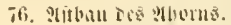

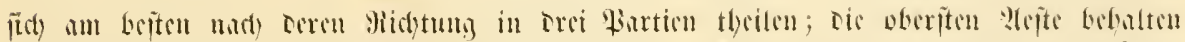

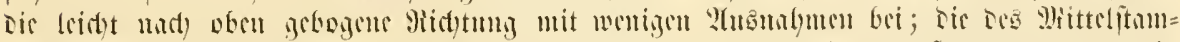

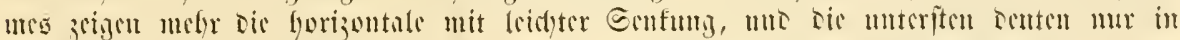

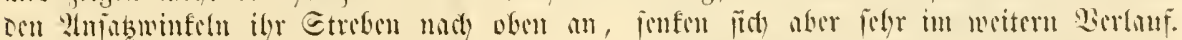

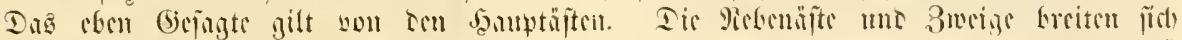

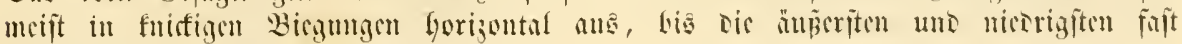
beriblymingen. 


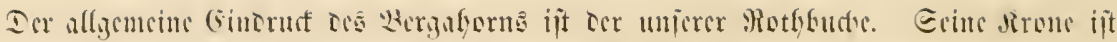

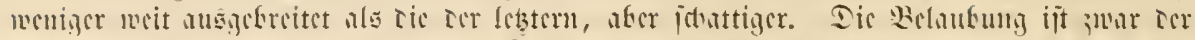

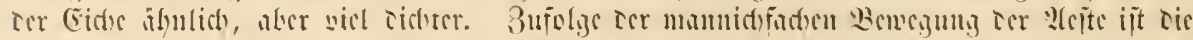

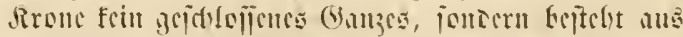

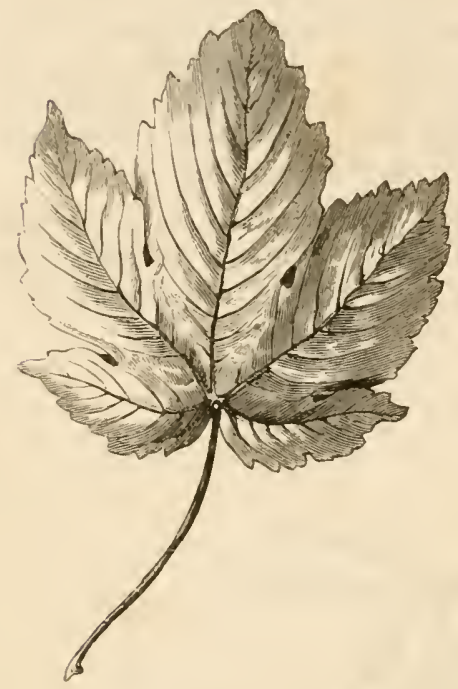

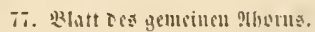

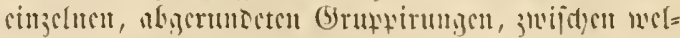
d)

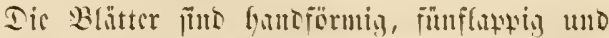

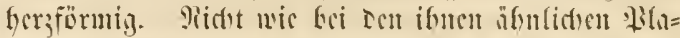

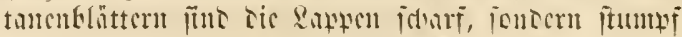

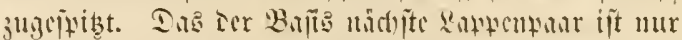

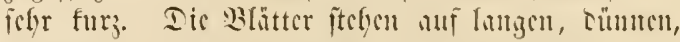

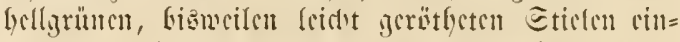

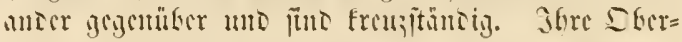

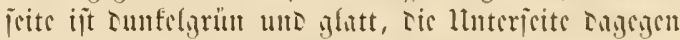

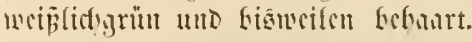

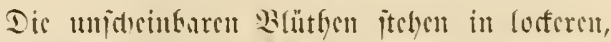

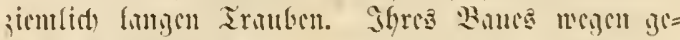

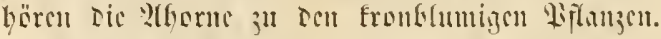

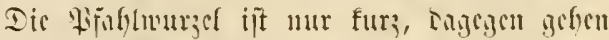
Lic friftigen Esitemururzeft meit ans. Esften tre=

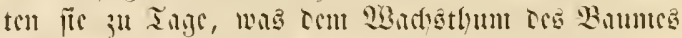

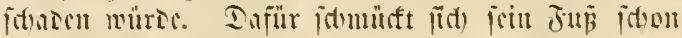

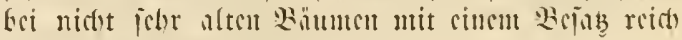
belaubter Etorfansidalinge.

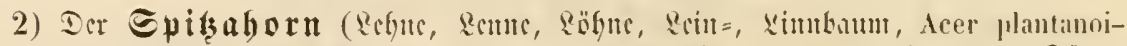

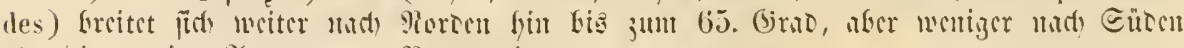

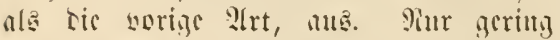

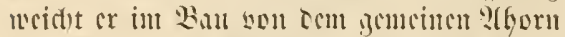

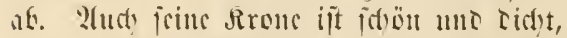

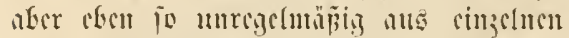

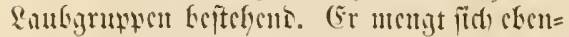
folla unter Bind)en uno (sidyen, jecod), in

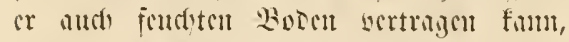

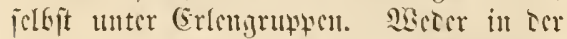

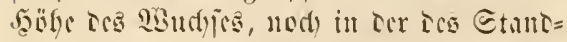

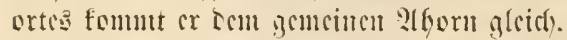

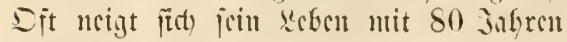
id)an tom (5ntes 3 th.

Dor Etorfmaidtlan ift ned frecu= tencer, unt boitefot ans lantgen, id)lunfen, gernecn Ed)oilen.

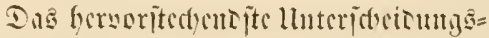
merfmal wor icm worizen 9 (horn ift sic

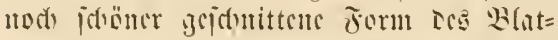
tras. Dis Ratpen futto nod bogiz nus=

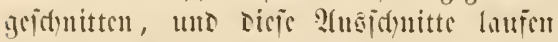

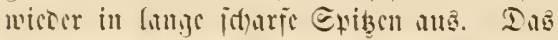

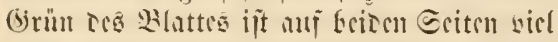

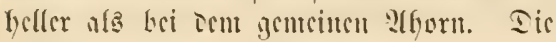
withtbon ftefon in Dolten.

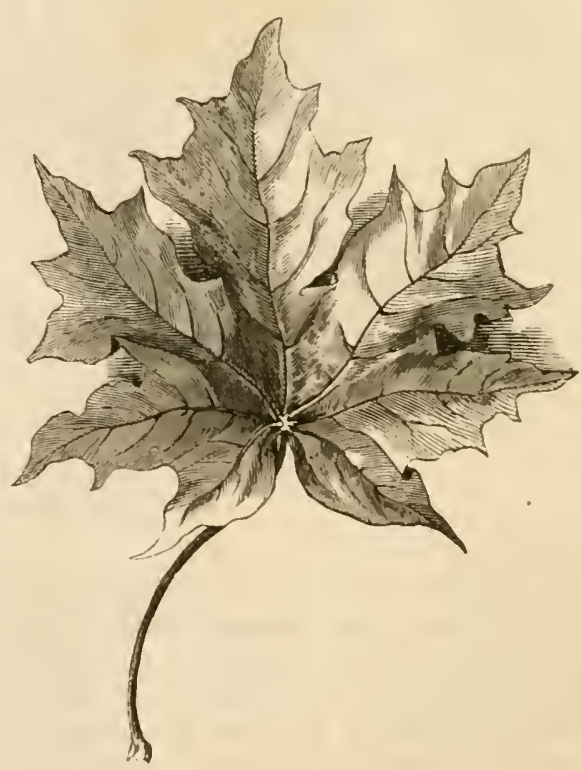

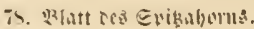




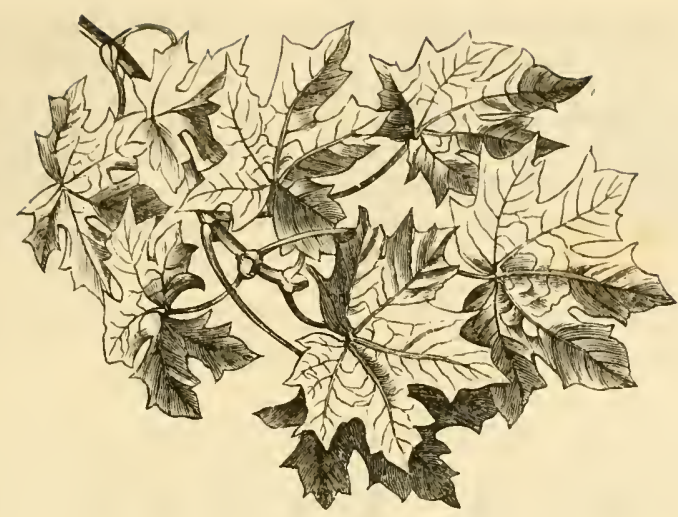

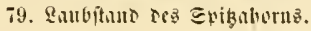

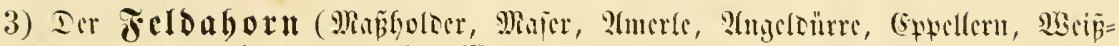

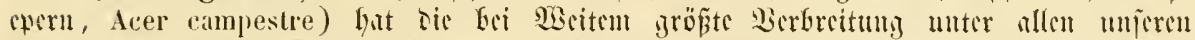

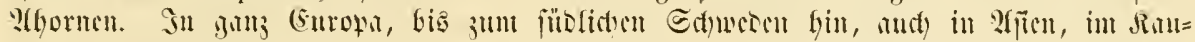
falus tuto Rlcinafien ift or befumut.

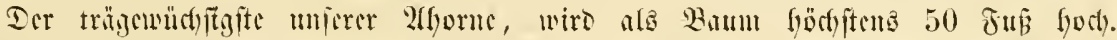

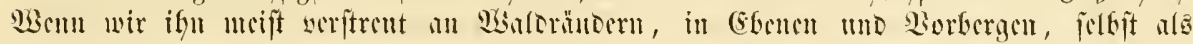

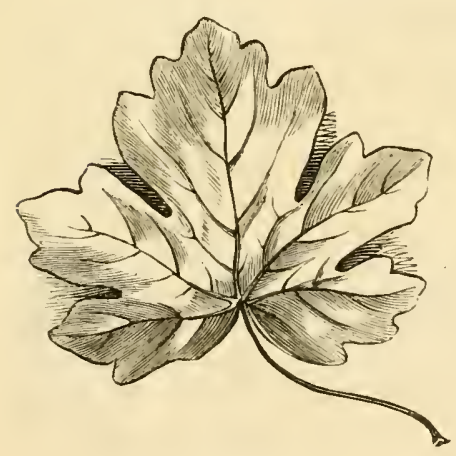

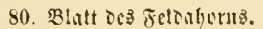

3weige Ler Rorfrüfter (Ulmus stlberosa) überzogat jüt.

Dic Blintter finto noll ver= idjicomem Edtuitt, fult ticf, fuld id)wad) fïnflaptig, sic stri nor= ocren senpen melyeremal ftumpf rimgerdututten uno gezälyt. Dic ticf geluppten glcid)en Denent gemtinch Miscigorns (Crataegus oxyacantha), Dodh fint iffre Eticle

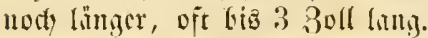
Se cin Fiar terielfen ftefen fist

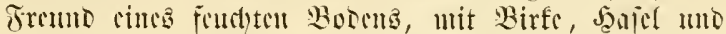

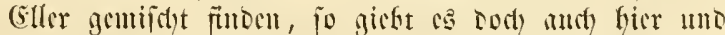

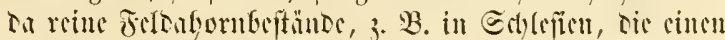
Simornef wie serfünmerte (sid)

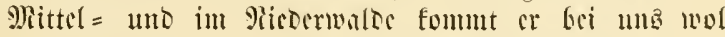

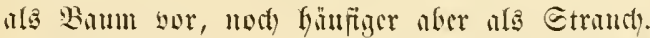

Dor Gtamm iff fein anfigriffen uno geffelidygrm,

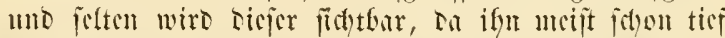

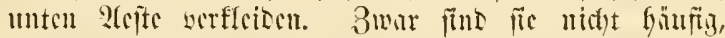

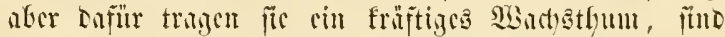

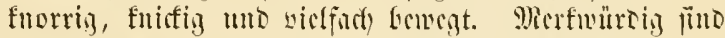

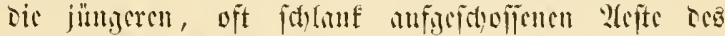

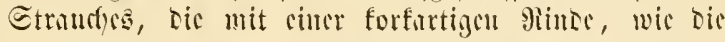

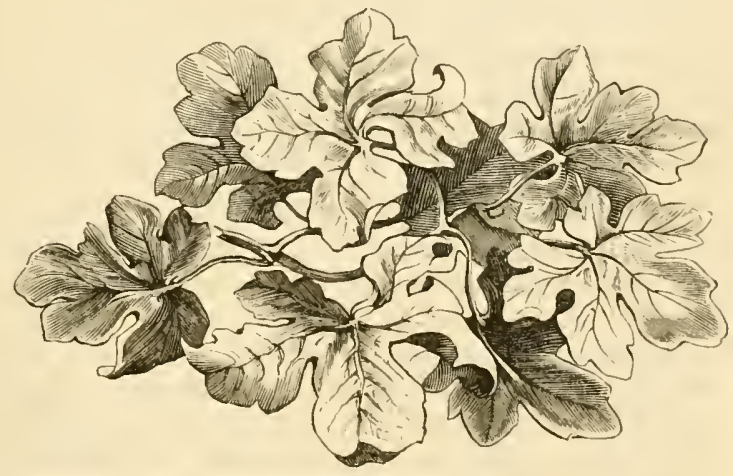

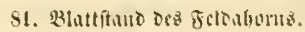




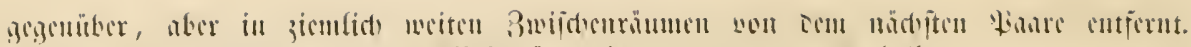

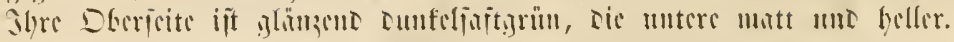

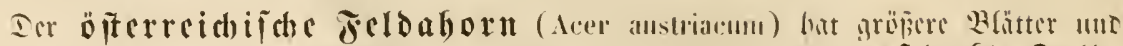

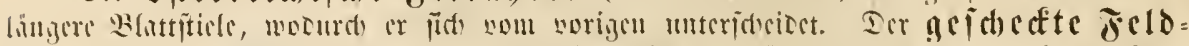

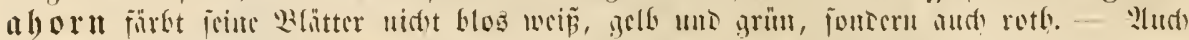

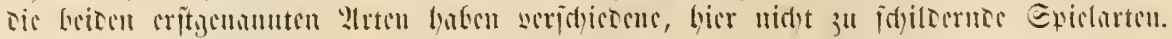

\section{(1) $i \mathfrak{i}$ 近 $i \mathfrak{n}$ ไั}

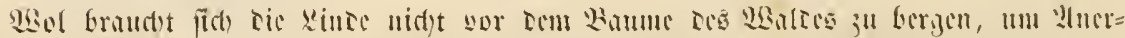

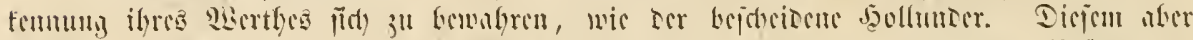

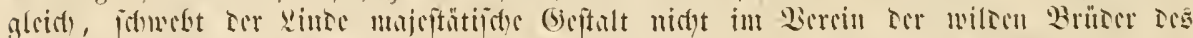

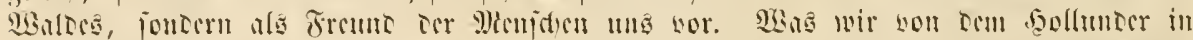

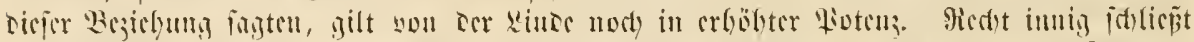

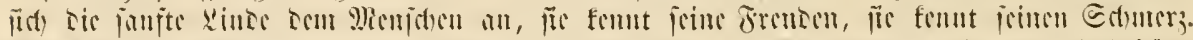

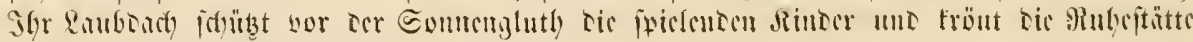
tris grlicbten Tramors.

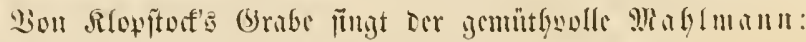

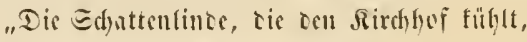

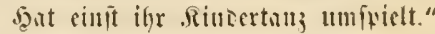

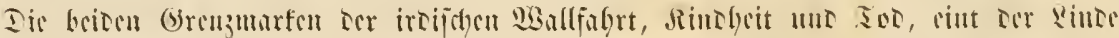
Edinttu.

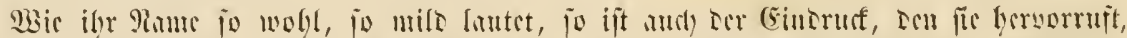
fanft uno mile. Mit jerer gemütbollen Grimmuny barmonirt ibr Egarnfter; Errejun=

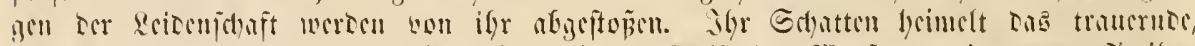

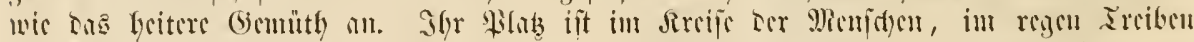

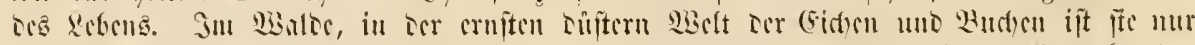

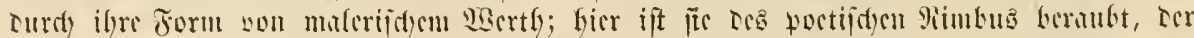

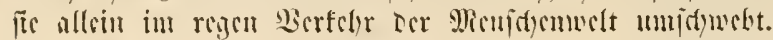

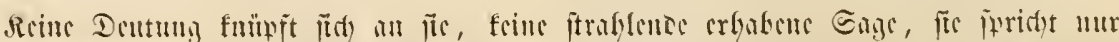

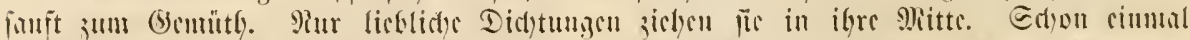

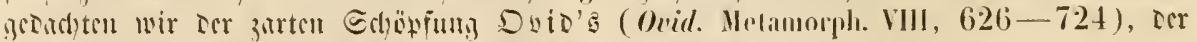

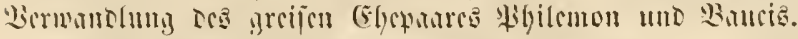

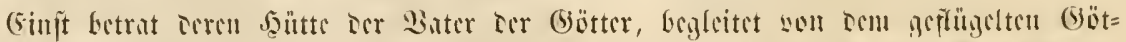

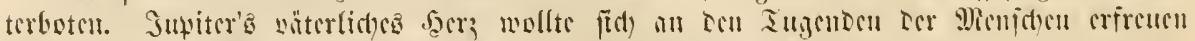

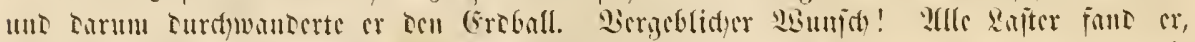

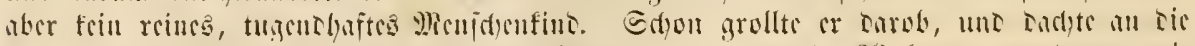

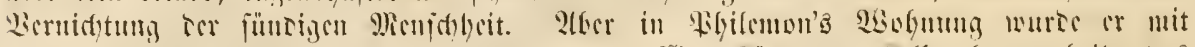

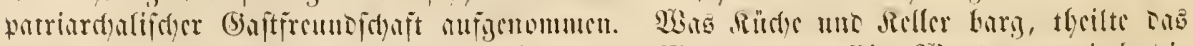

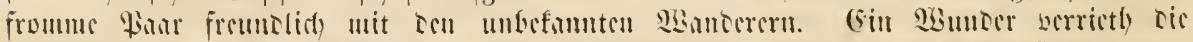

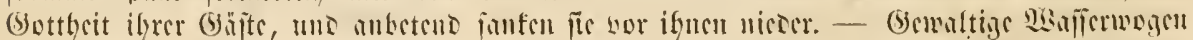

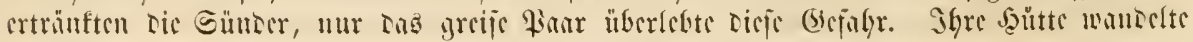




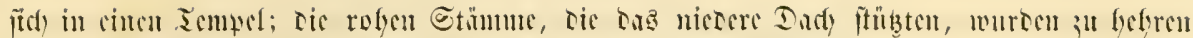

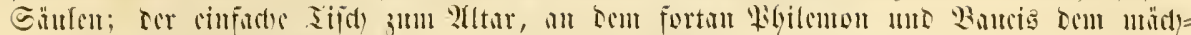

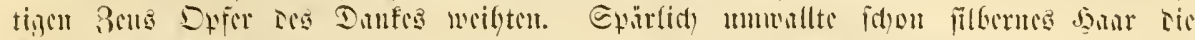

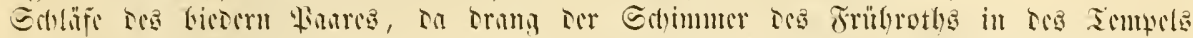

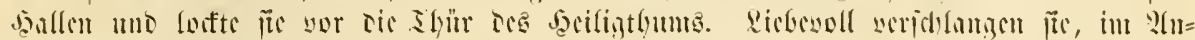

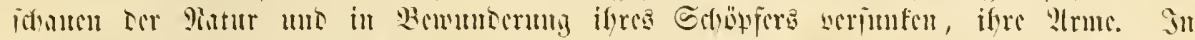

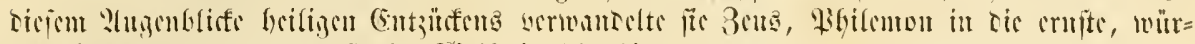

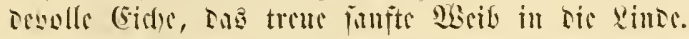

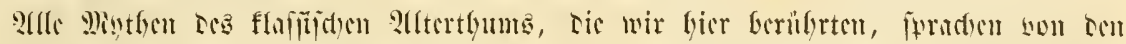

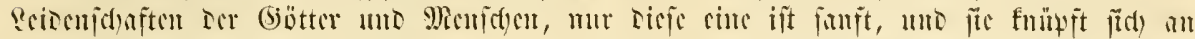
urjere sinte.

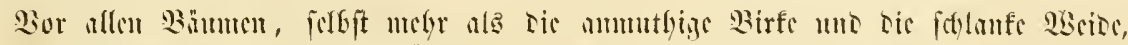

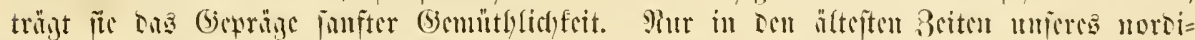

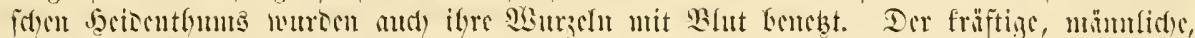

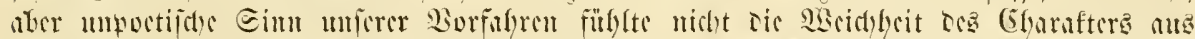

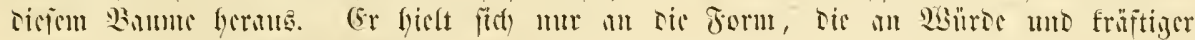

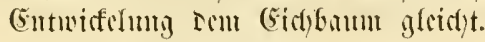

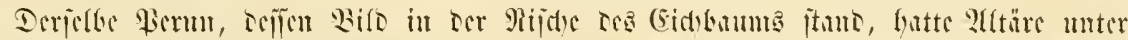

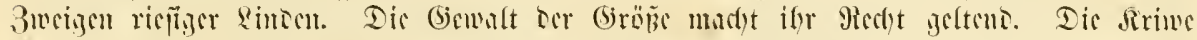

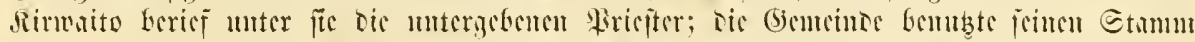

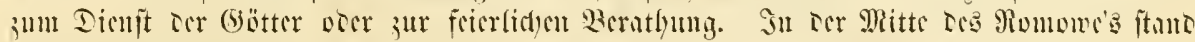
cheri jo gut ric since, alo ric Gidyc.

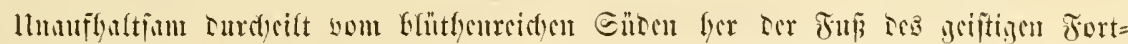

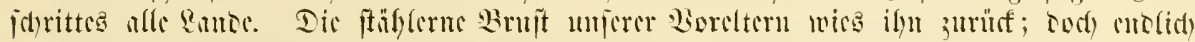

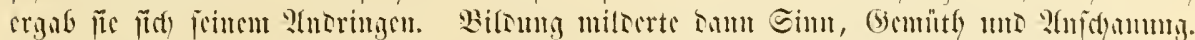

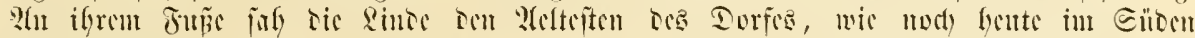

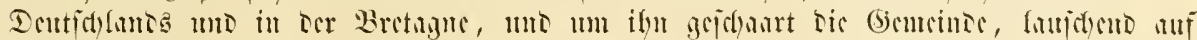

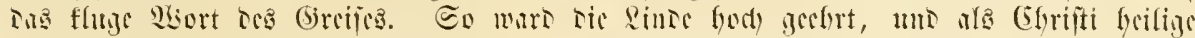

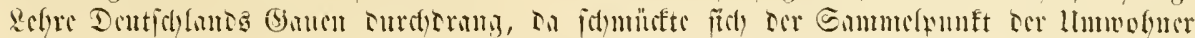

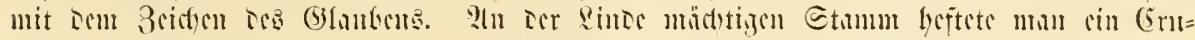

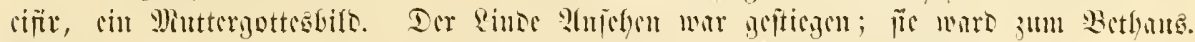

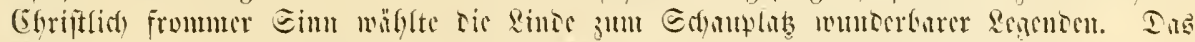

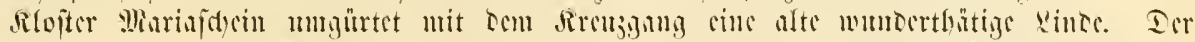

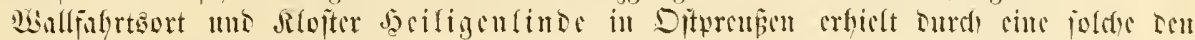
?hantur.

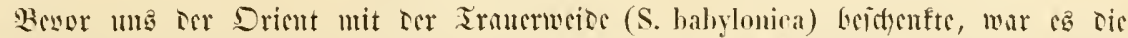

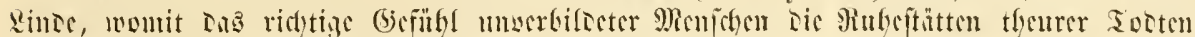

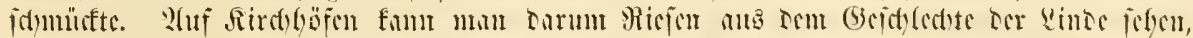

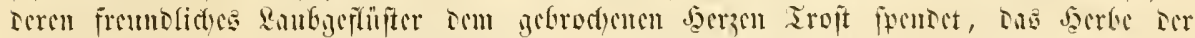

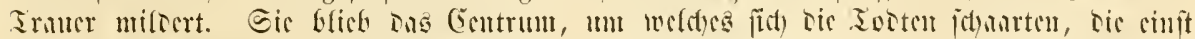
im Refoen jie fröblict) umjubslt forten.

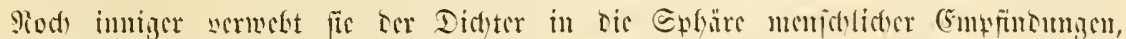

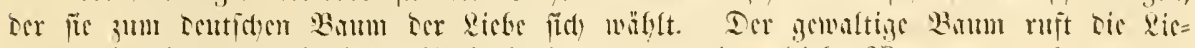

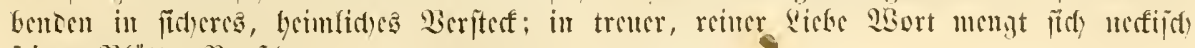

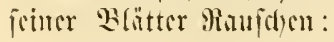

13d) far bei grüncr since

\$lit meinem trituten Rinte;

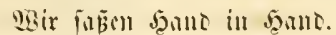

( 


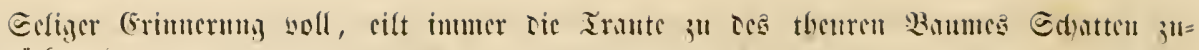
rïrf 3 unt

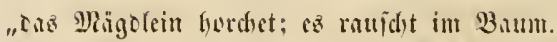 \\ Eibnent, \\ DSithneno

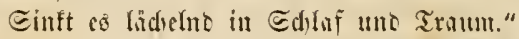

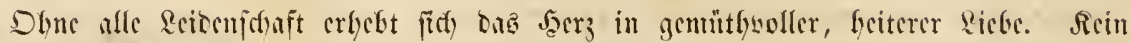

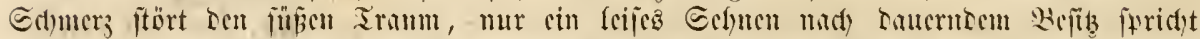

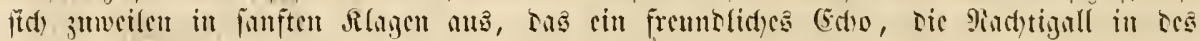

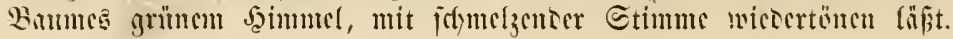

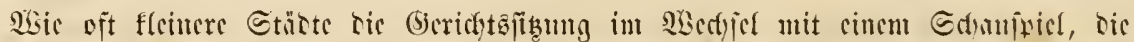

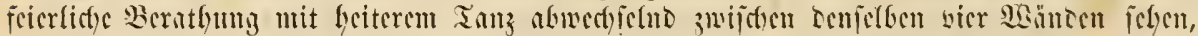

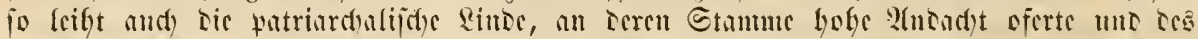

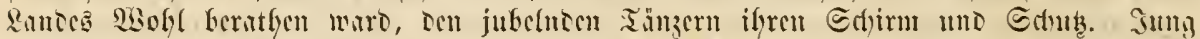

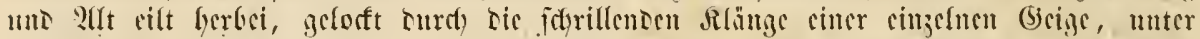

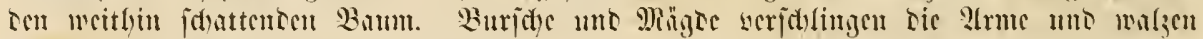
joblent um oor zinte Garift.

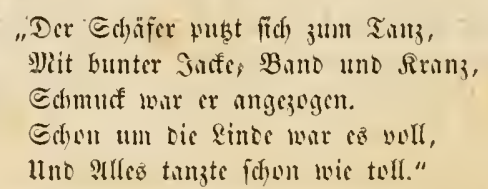

(Goctlyc.)

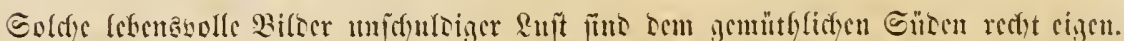

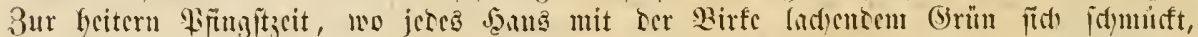

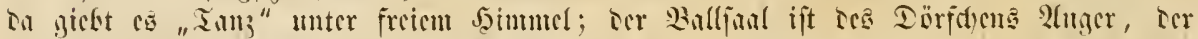

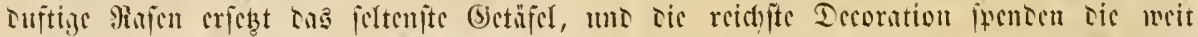

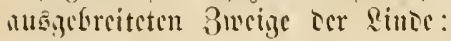

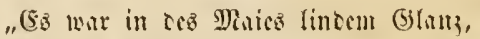

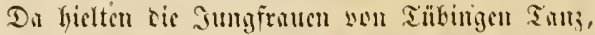

Eic tanjten, uno tamzten wel allimunal,

llu cine sinte iin grünen Thal."

\title{
(Э. Rerncr.)
}

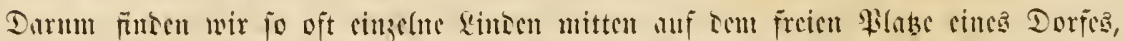

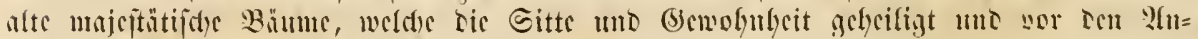

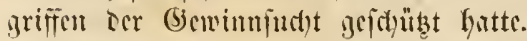

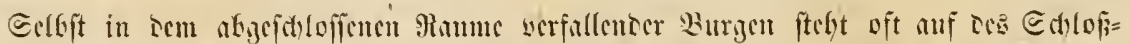

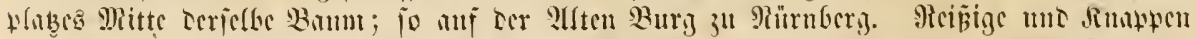

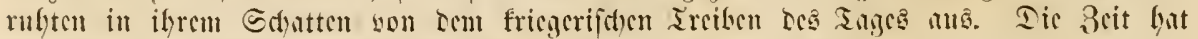
ICs Buratgors fübucu Bogen zertrüntmert,

$$
\begin{aligned}
& \text { "in ben osen Fenterhoblen }
\end{aligned}
$$

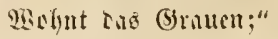

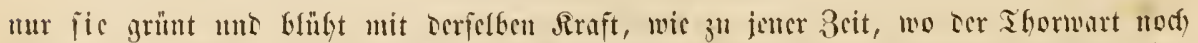

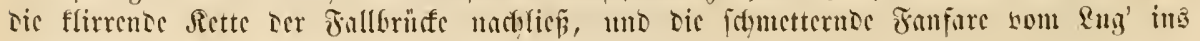

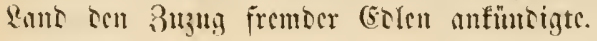




\section{went}

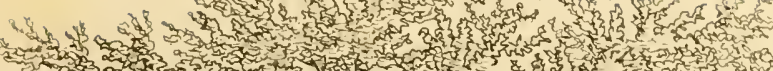

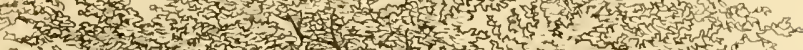

math

math

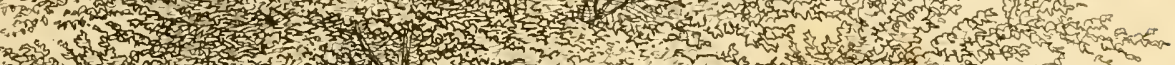

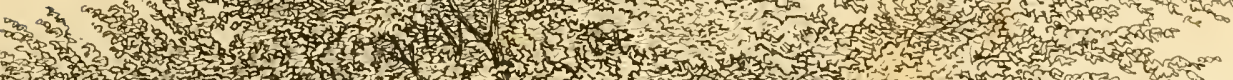

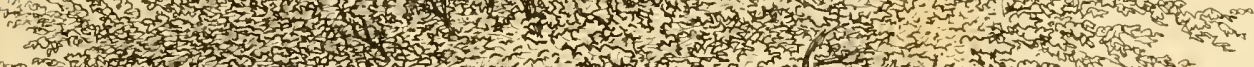

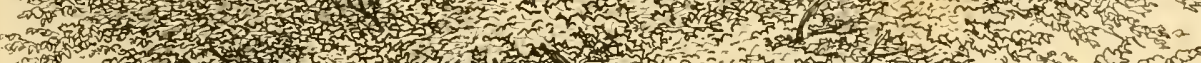

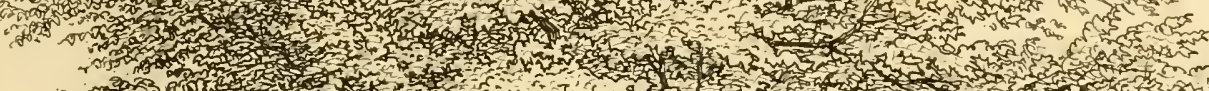

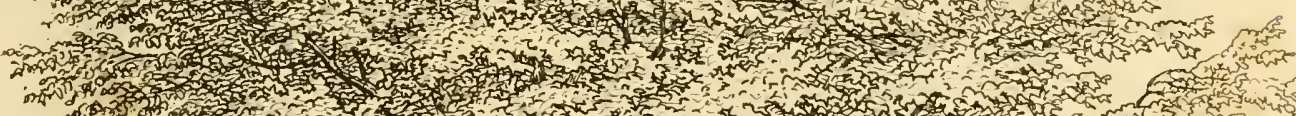

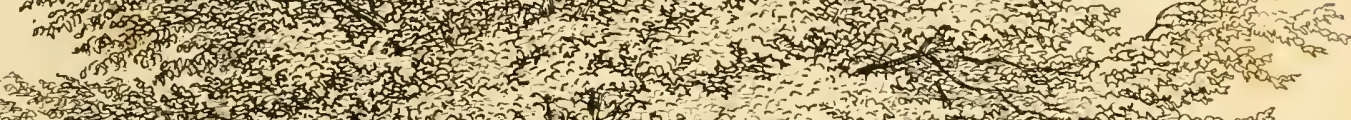

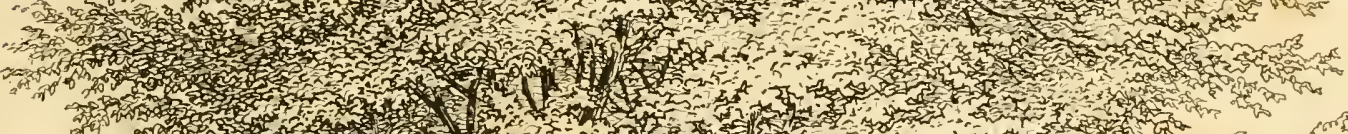

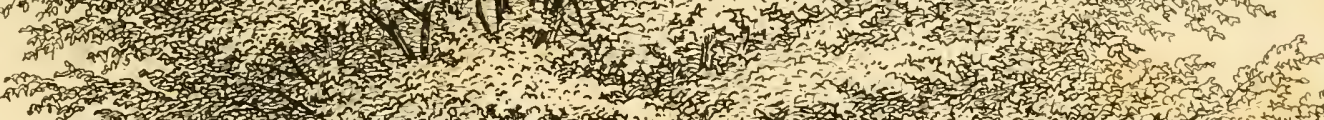

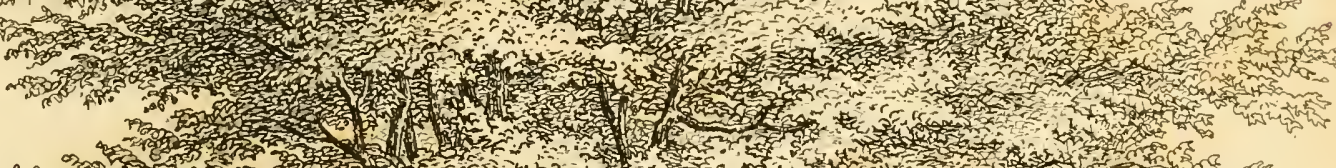

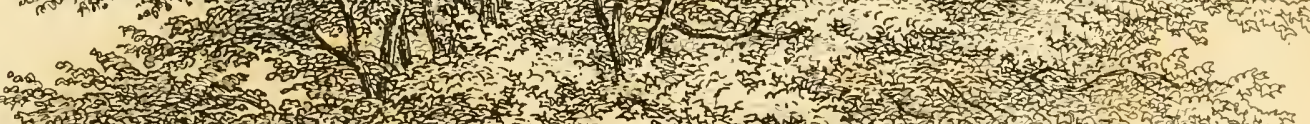

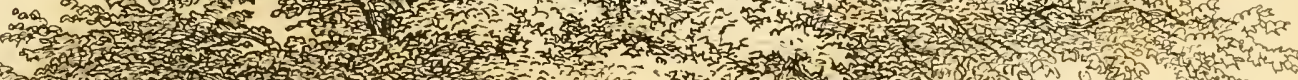
minn

W

20.5.

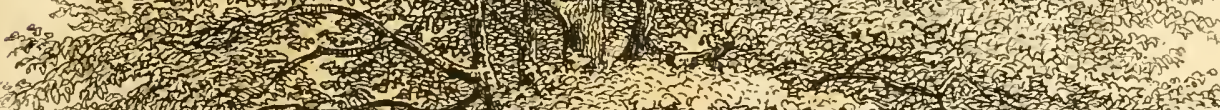

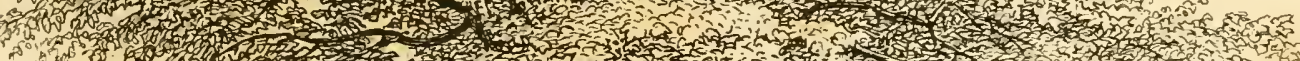

major. r.

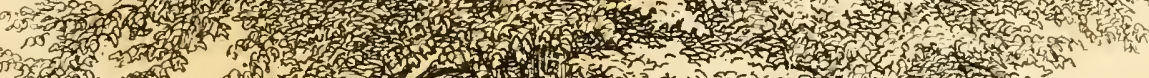

(1) 0 .

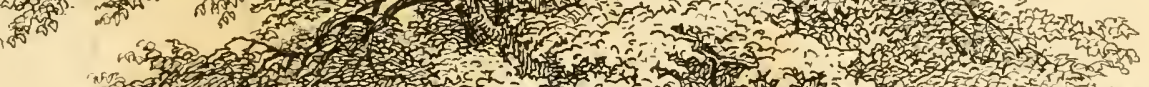
m.

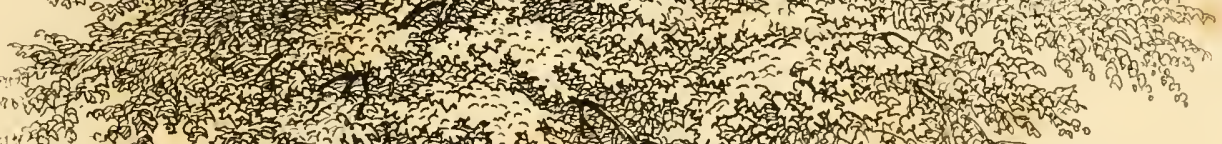

r.

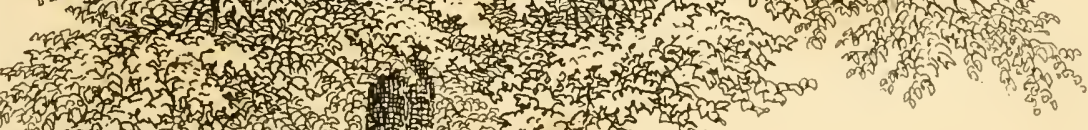
30

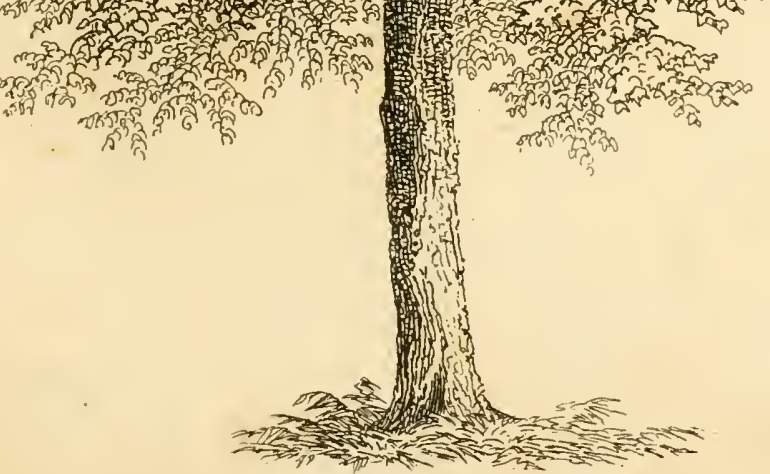



"Dort itelyen alo whithter, cingelullt in Erimum,

Dir alten, bürft'gen Eintentüume."

(6. (Sicibel.)

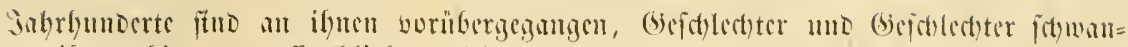

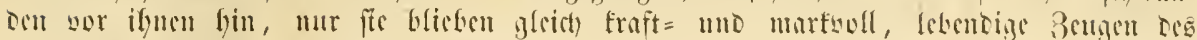
Eraftuollen Mittelnlters.

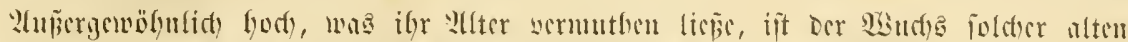

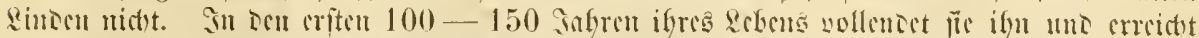

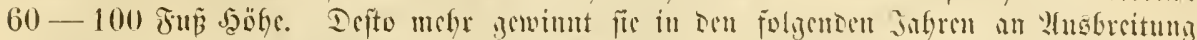

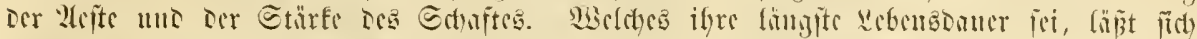

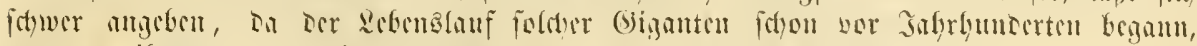
wo mon iforer nod) meniger adtets.

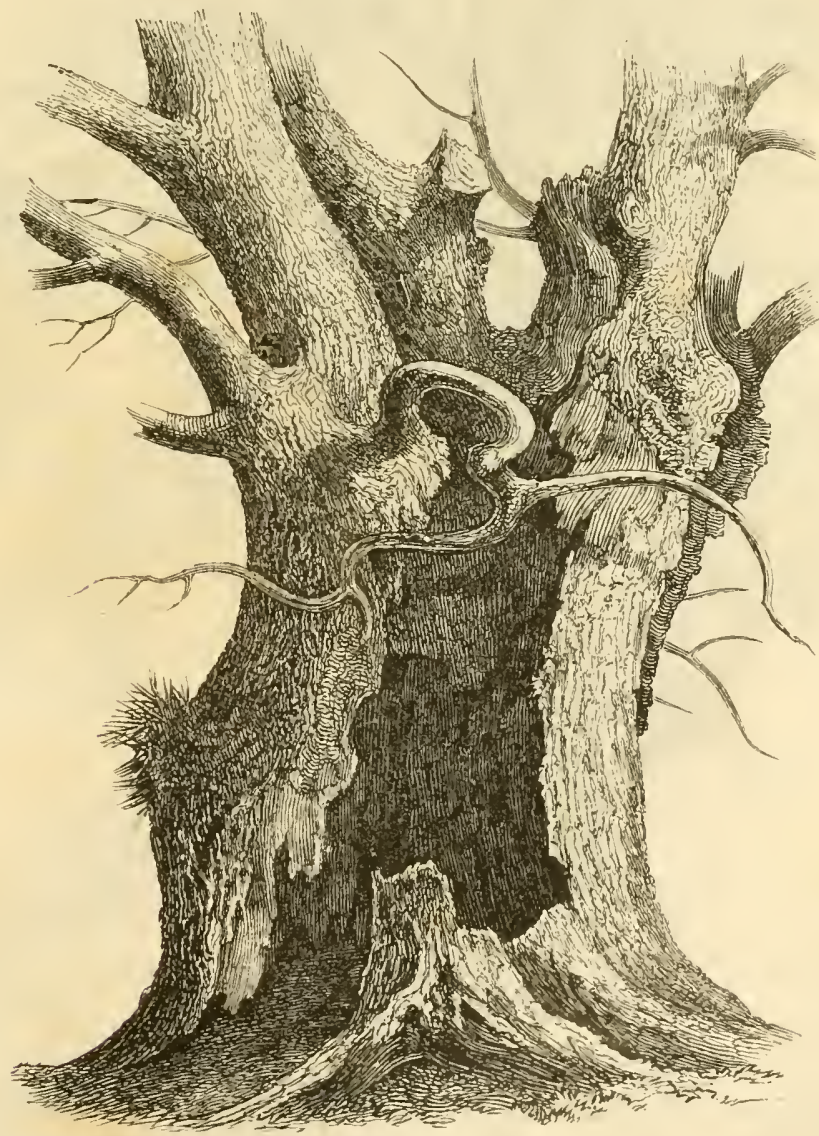

\$2. Etanm for winte.

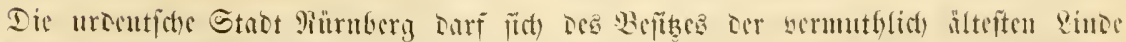

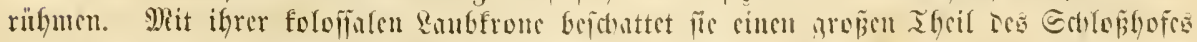

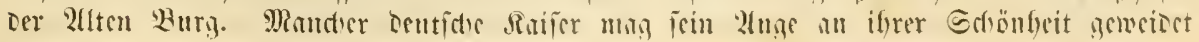




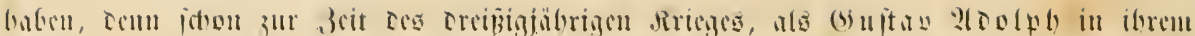

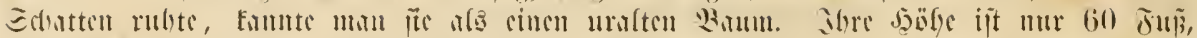

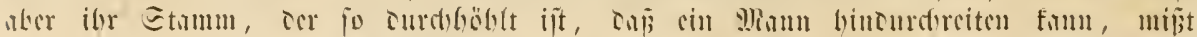

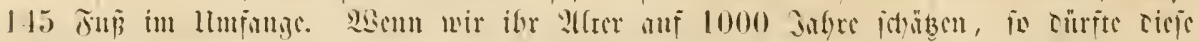

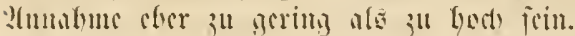

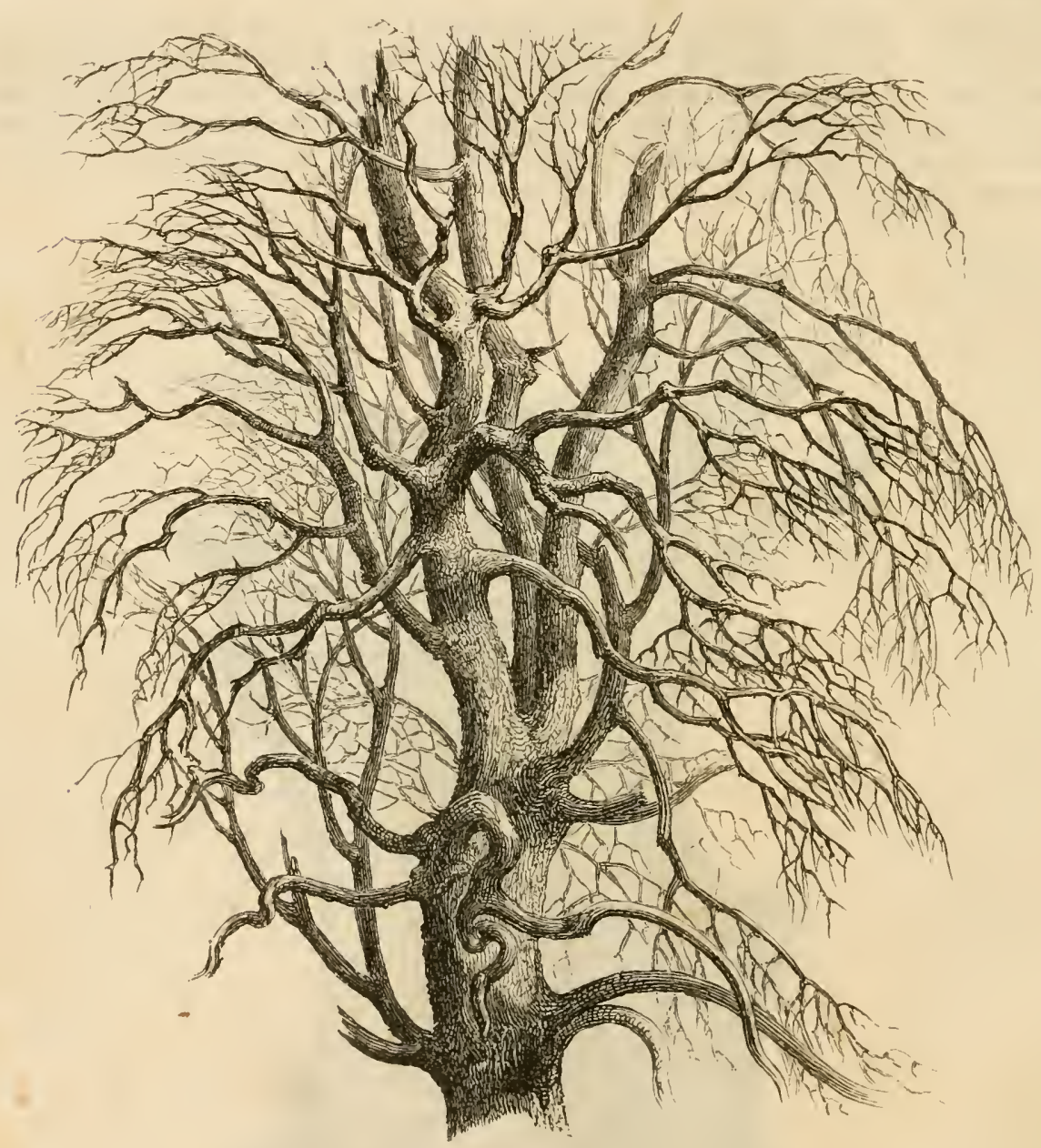

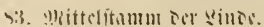

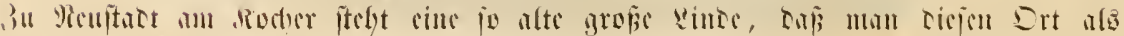

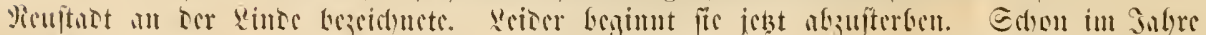

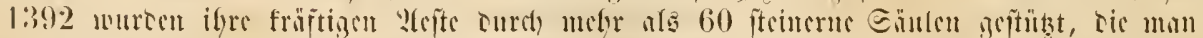

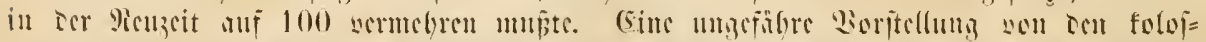

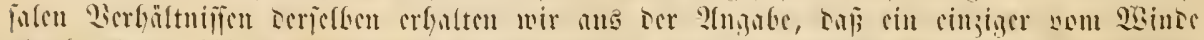

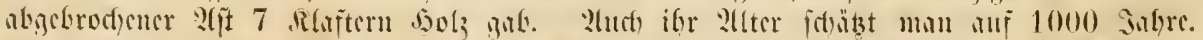

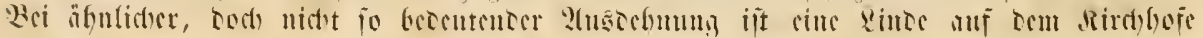




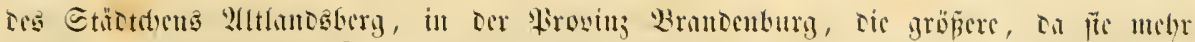

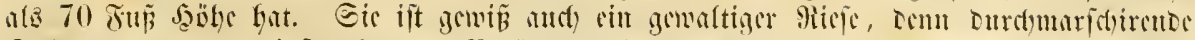

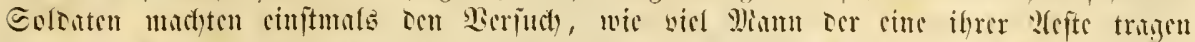

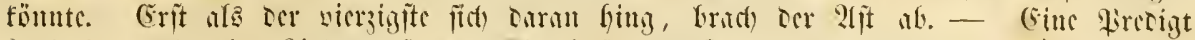

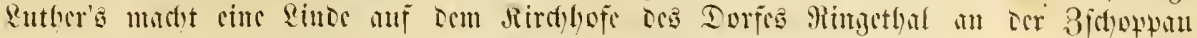

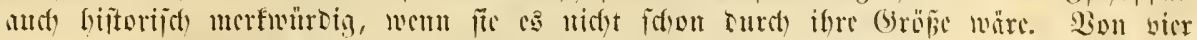

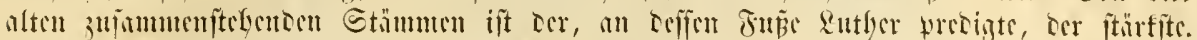

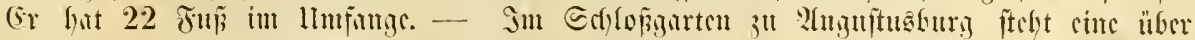

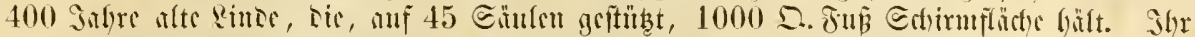

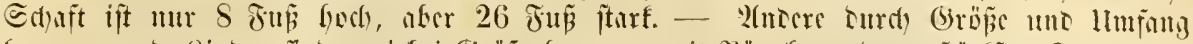

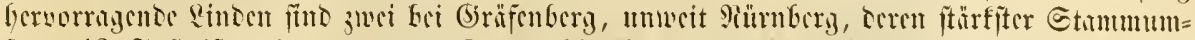

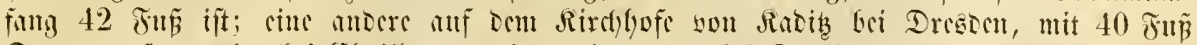

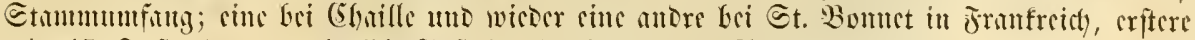

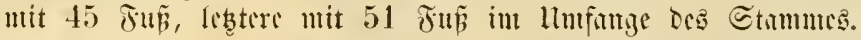

Bon Den mancherlei 2 (ren mo Barictäten menten mir nur bic beiden berworite=

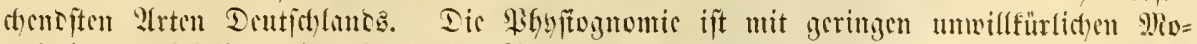

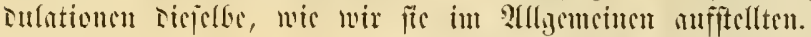

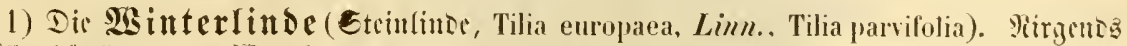

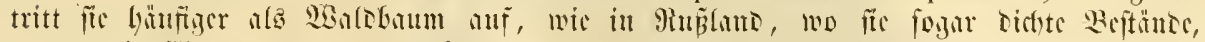

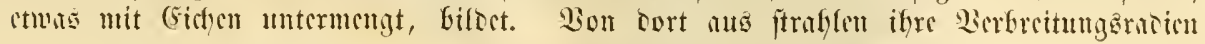

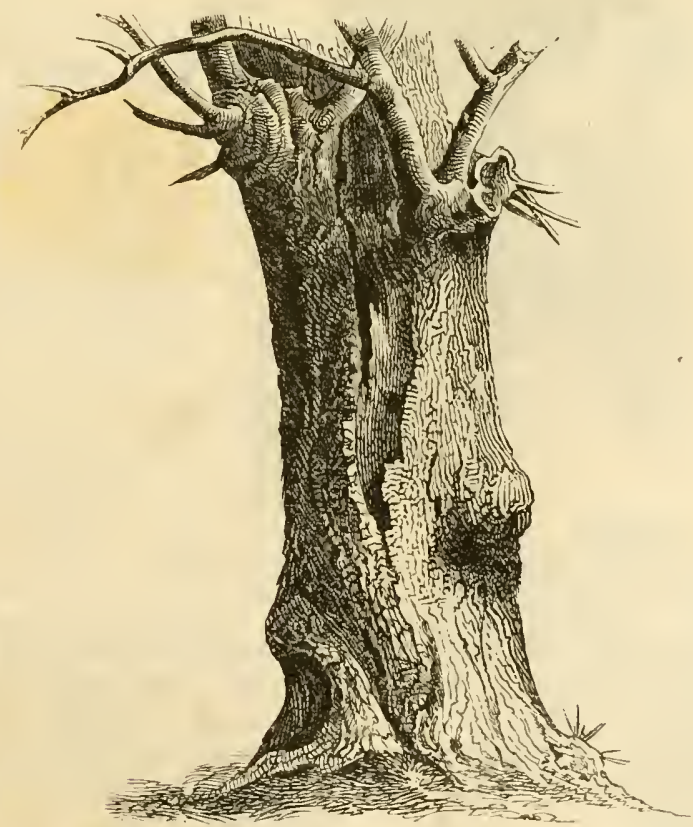

S4. Etumm oer $\mathfrak{B}$ interdinde.

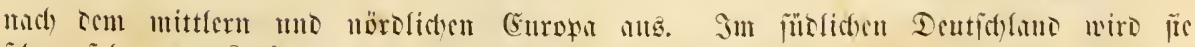

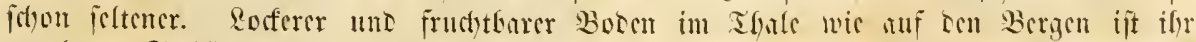

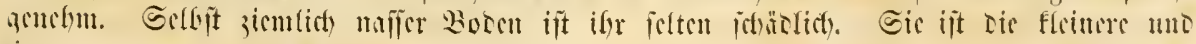




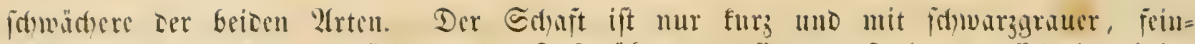

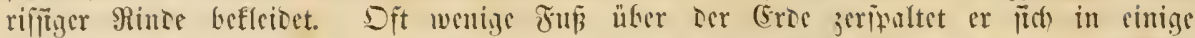

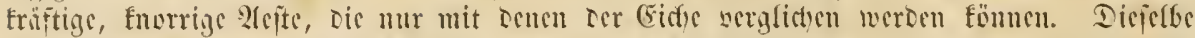

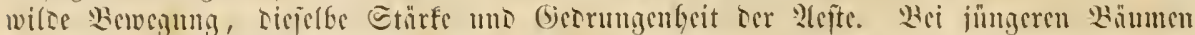

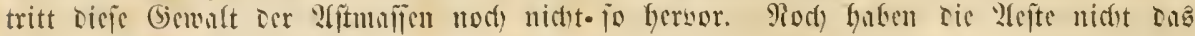

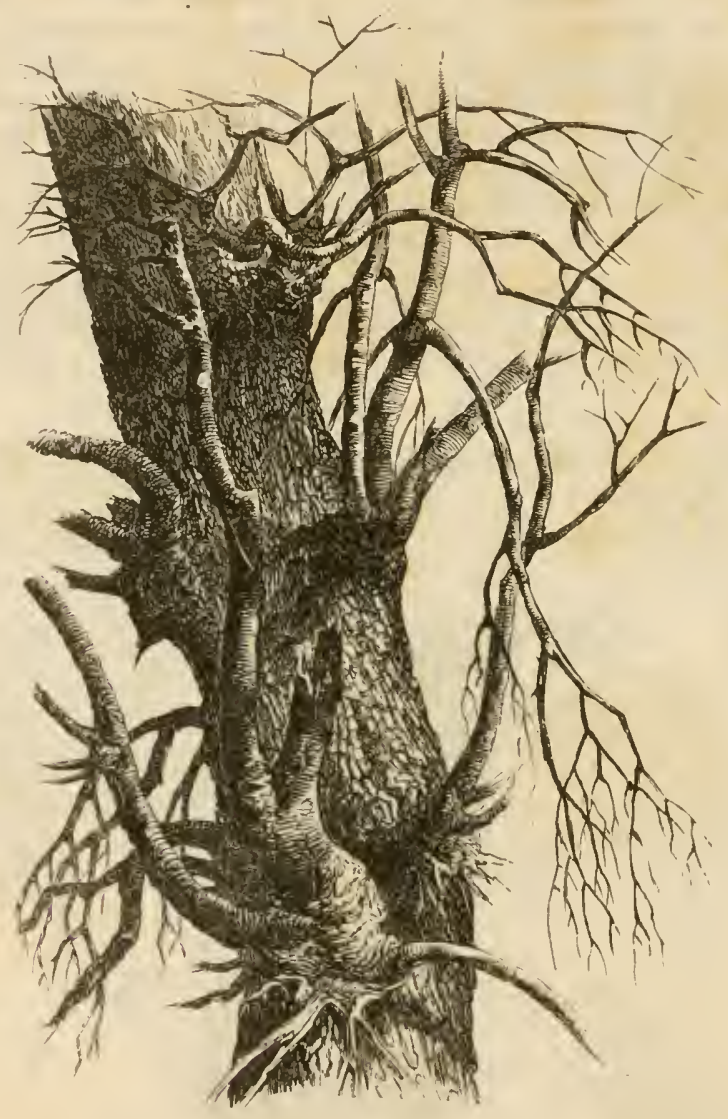

55. Dittelitamut unc atitban ofr 2ginterlute

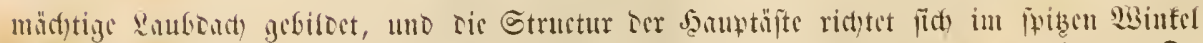

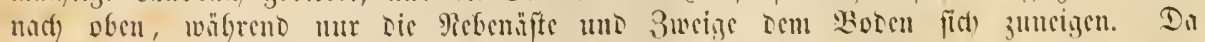

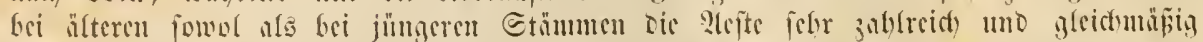

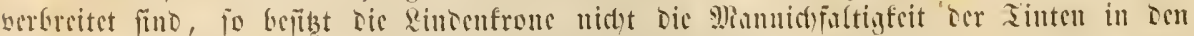

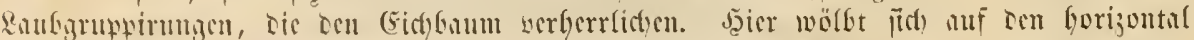

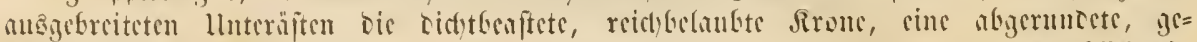

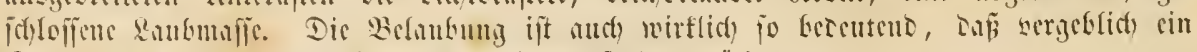

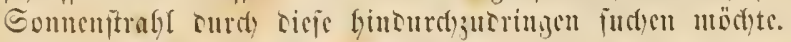

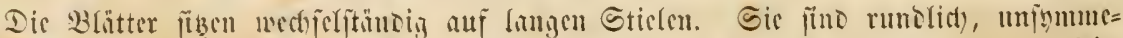

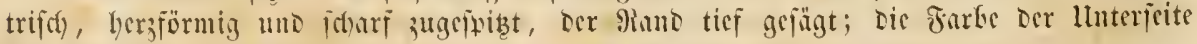
lid)ter als Die tor Doericite. 


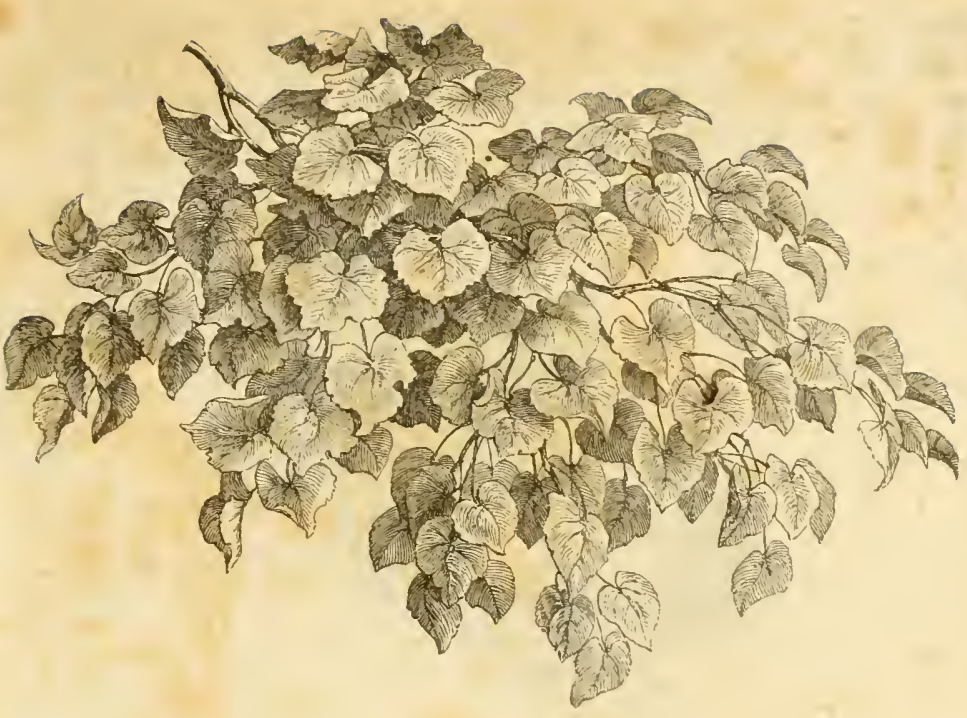

86. Raubgruple Der 2Binterlints.

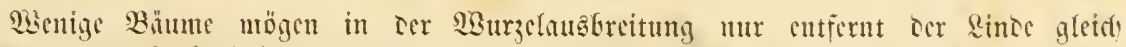

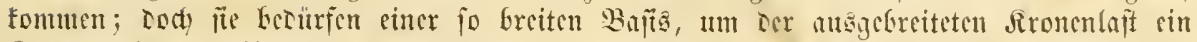
(bigengenicft ju bisten.

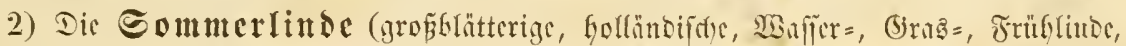

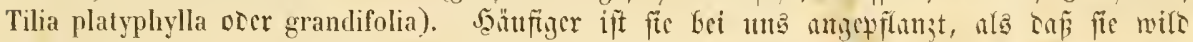

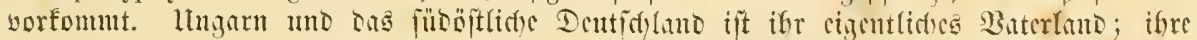

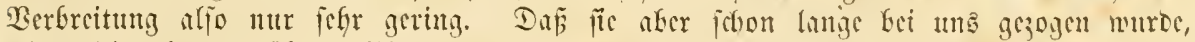

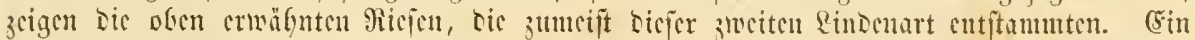

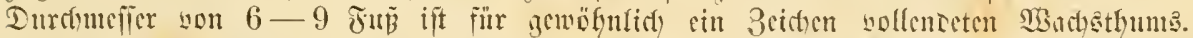

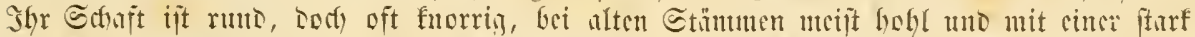

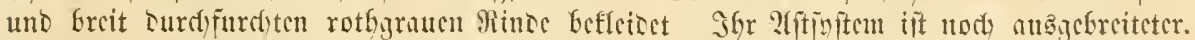

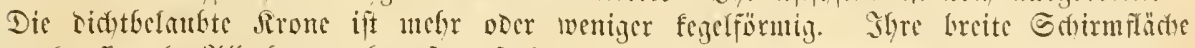

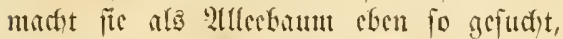
wie man fie ibres @et)attens mezen fonjt

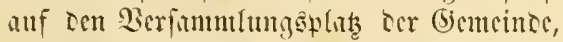
sor cas Thor Der Buingfury fiflanste. Da ifre Blïter Getentento sröper fino uno in Derielben Menge aen Baum fortunifen, fo ije Diejer Sinte Beluubung nod) Sictuter. Dic Blätter find forjförmig ttno forarf 3ugeppizet, uno necift fo breit als lang. Jhr Rand ift fein gezifgnt, dic Socricitc if lebhaft grin und bic llntericite nur isenig heller. Die fternförmigen Blütben, weldse bie Rinde unter bie fronblumigen Pilanzen reiben, fteben zul orei it Dol= Den, Die nebit cinem grüngelben Rebentilatt son Entzettform auf cinem jefr langen

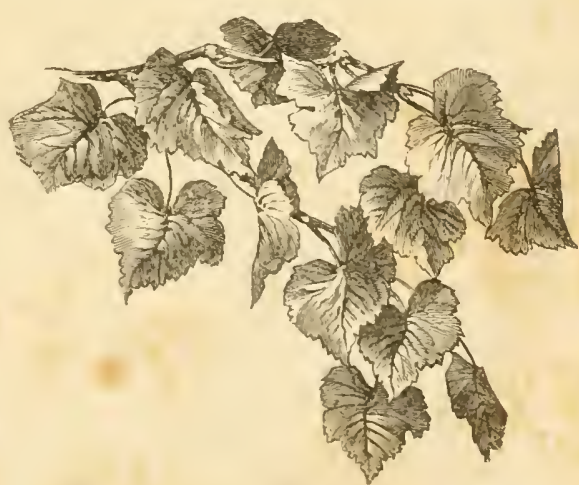

87. Rlätterftans ter Sommerlints. 


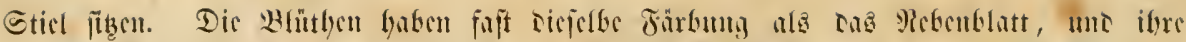

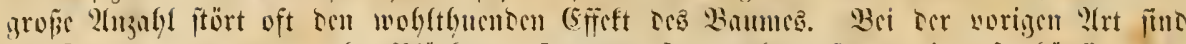

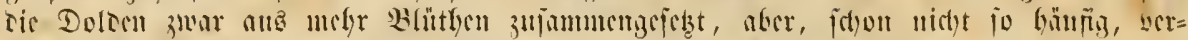
bergen fie fitc) metyr unter bou Raube.

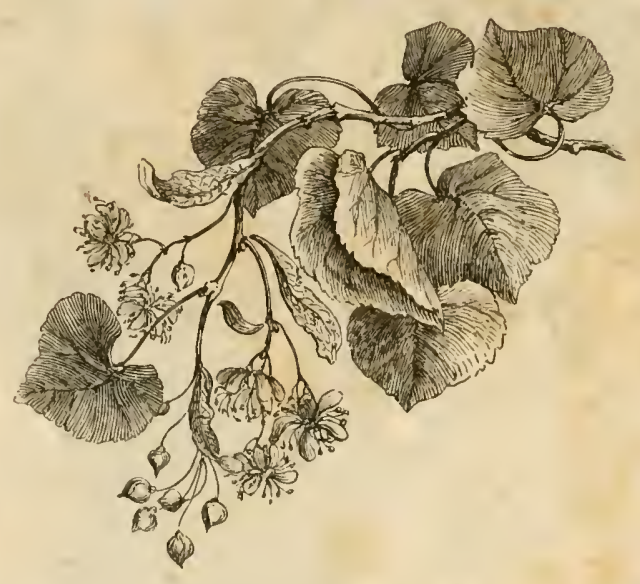

85. Blülle Dor Eomutrinte.

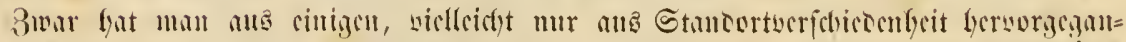

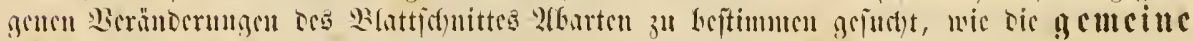

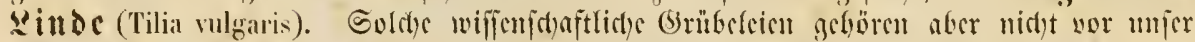
ซormm. 




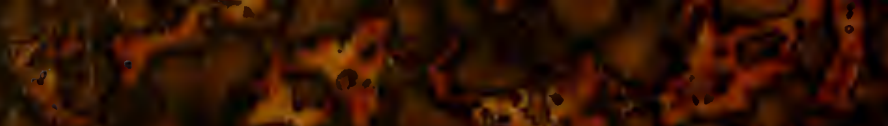

2.

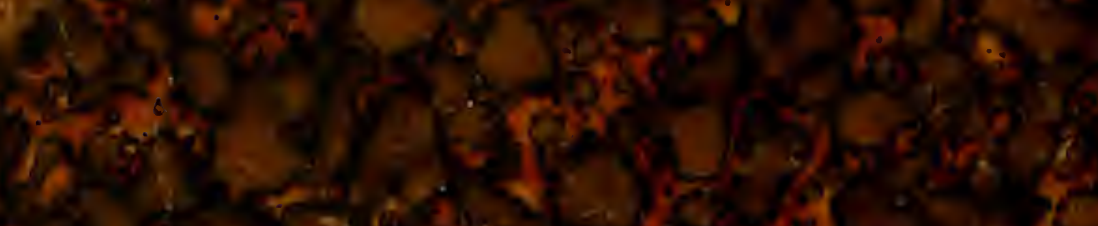

(1) 4 a

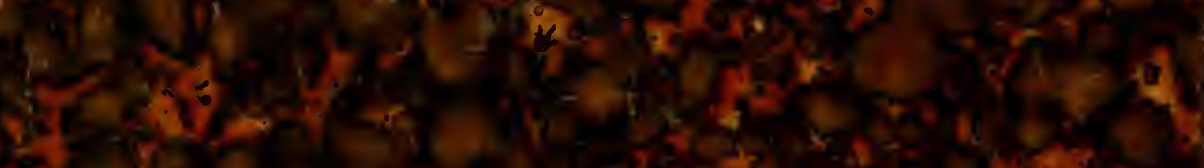

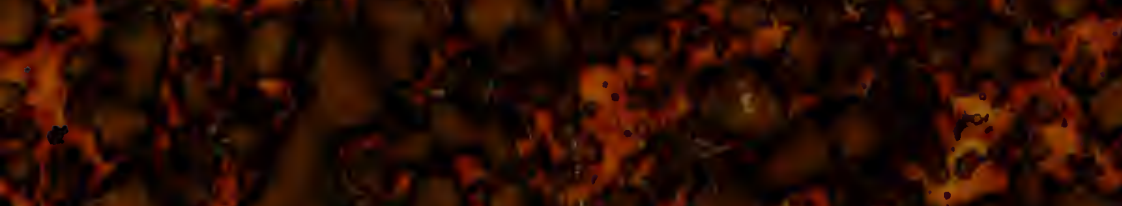

X.

t.

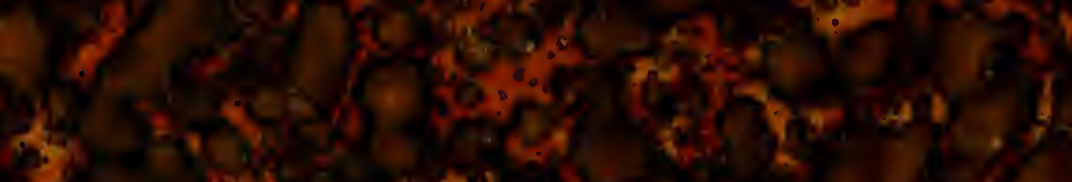

2.

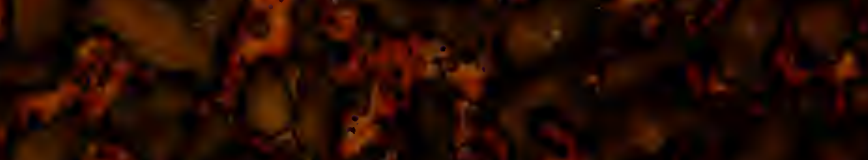

2.5

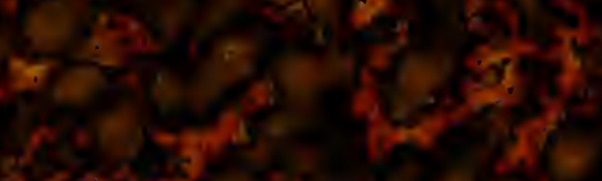

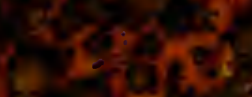

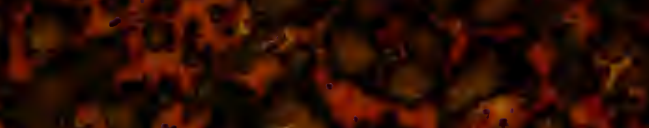

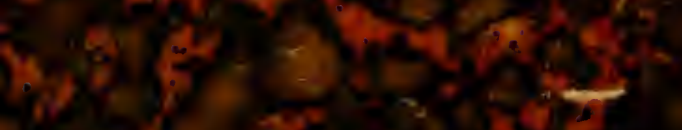

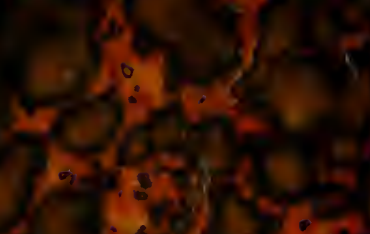

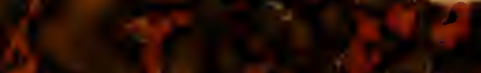

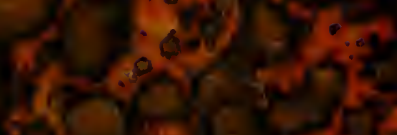

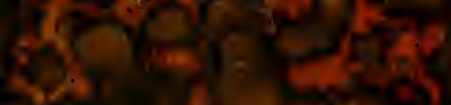

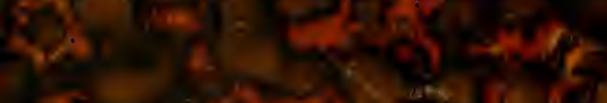

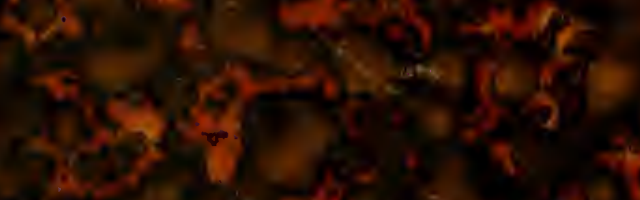

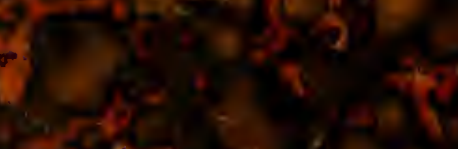

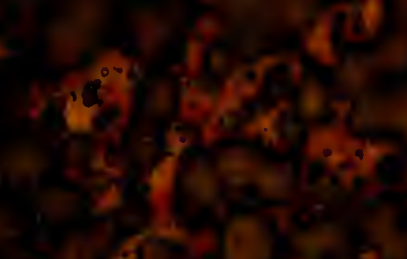

$x_{4}$ 